\begin{tabular}{|l|l|}
\hline Report Title & $\begin{array}{l}\text { A Data Fusion System for the Nondestructive } \\
\text { Evaluation of Non-Piggable Pipes }\end{array}$ \\
\hline Type of Report & Semiannual Technical Progress Report \\
\hline Reporting Period Start Date & April 1, 2004 \\
\hline Reporting Period End Date & September 30, 2004 \\
\hline Principal Author(s) & $\begin{array}{l}\text { Dr. Shreekanth Mandayam (PI), } \\
\text { Dr. Robi Polikar (Co-PI), } \\
\text { Dr. John C. Chen (Co-PI) }\end{array}$ \\
\hline Date Report was Issued & January 2005; Revised February 2005 \\
\hline DOE Award Number & DE-FC26-02NT41648 \\
\hline $\begin{array}{l}\text { Name and Address of the } \\
\text { Submitting Organization }\end{array}$ & $\begin{array}{l}\text { Rowan University } \\
\text { 201 Mullica Hill Road } \\
\text { Glassboro, NJ 08028 }\end{array}$ \\
\hline
\end{tabular}




\section{Disclaimer}

"This report was prepared as an account of work sponsored by an agency of the United States Government. Neither the United States Government nor any agency thereof, nor any of their employees, makes any warranty, express or implied, or assumes any legal liability or responsibility for the accuracy, completeness, or usefulness of any information, apparatus, product, or process disclosed, or represents that its use would not infringe privately owned rights. Reference herein to any specific commercial product, process, or service by trade name, trademark, manufacturer, or otherwise does not necessarily constitute or imply its endorsement, recommendation, or favoring by the United States Government or any agency thereof. The views and opinions of authors expressed herein do not necessarily state or reflect those of the United States Government or any agency thereof." 


\begin{abstract}
The objectives of this research project are -

1. To design sensor data fusion algorithms that can synergistically combine defect related information from heterogeneous sensors used in gas pipeline inspection for reliably and accurately predicting the condition of the pipe-wall.

2. To develop efficient data management techniques for signals obtained during multisensor interrogation of a gas pipeline.

During this reporting period, Rowan University designed, developed and exercised multisensor data fusion algorithms for identifying defect related information present in magnetic flux leakage, ultrasonic testing, thermal imaging and acoustic emission nondestructive evaluation signatures of a test-specimen suite representative of benign and anomalous indications in gas transmission pipelines. Specifically, the algorithms presented in the earlier reports were augmented to predict information related to defect depth (severity).
\end{abstract}




\section{Table of Contents}

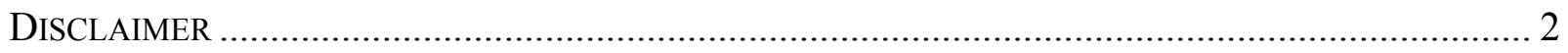

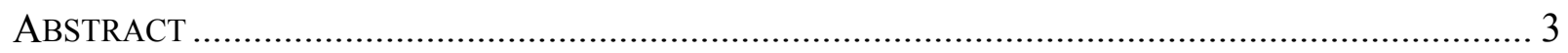

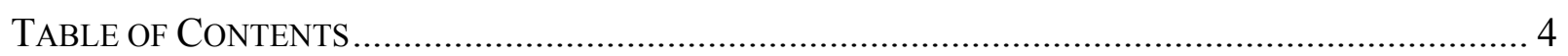

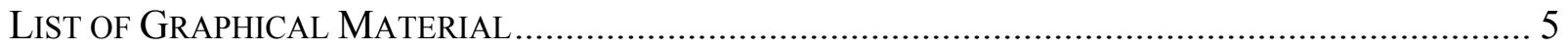

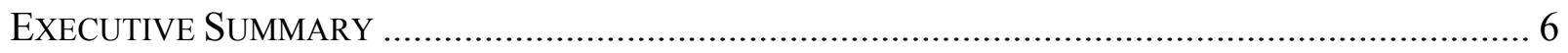

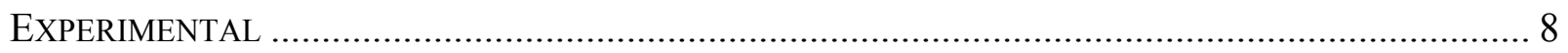

Task 3.0 - Design and Development Data Fusion Algorithms ....................................................................

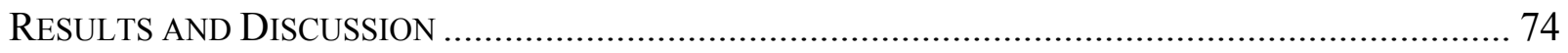

Task 3.0 - Design and Development Data Fusion Algorithms ......................................................................74

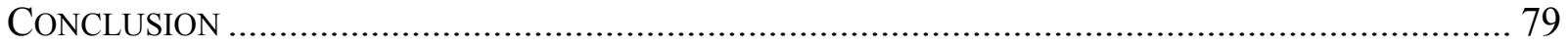

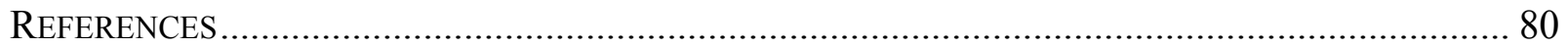

LIST OF ACRONYMS AND ABBREVIATIONS .................................................................. 81 


\section{List of Graphical Material}

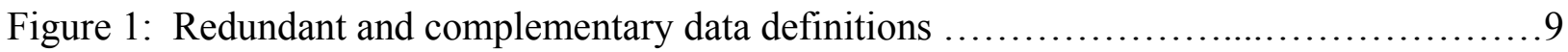

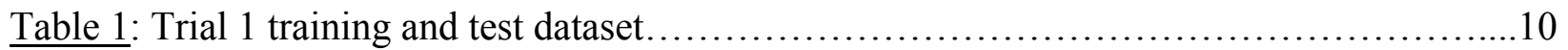

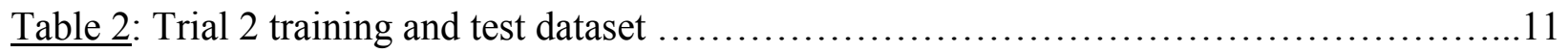

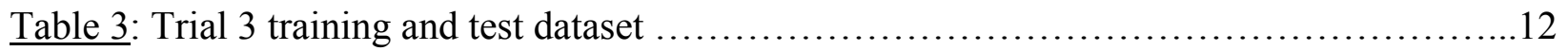

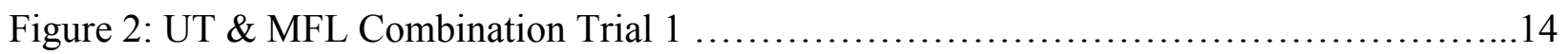

Figure 3: UT \& MFL Combination Trial 2 ............................................ 19

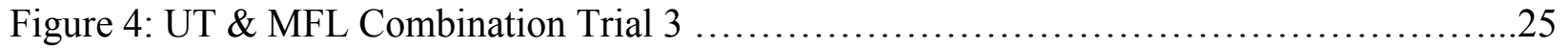

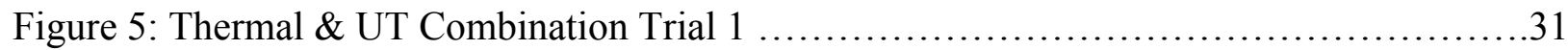

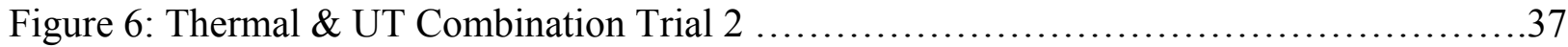

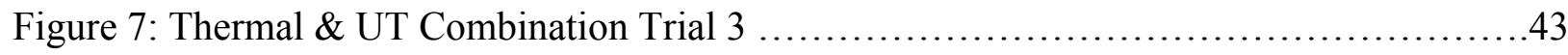

Figure 8: MFL \& Thermal Combination Trial 1 ......................................49

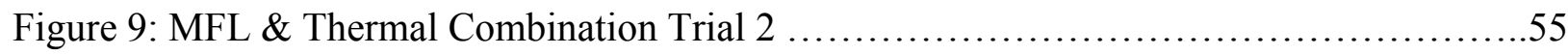

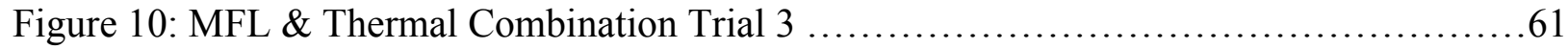

Figure 11: K-means clustering on AE data of uniaxial specimen with 0.16 " deep defect ......67

Figure 12: Parzen windows density estimation of each class separately $\ldots \ldots \ldots \ldots \ldots \ldots \ldots \ldots . \ldots 8$

Figure 13: Binary representation of Parzen windows AE images .........................68

Figure 14: Defect location representation from AE data ..............................69

Figure 15: AE location estimation plots for the data fusion algorithm ......................69

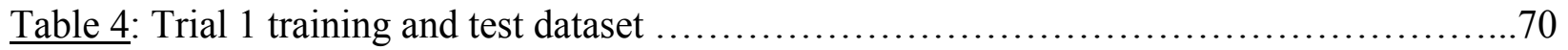

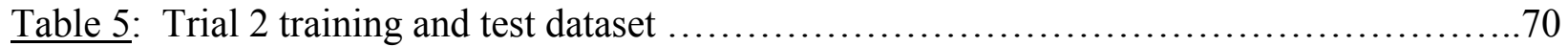

Figure 16: AE \& UT Combination Trial 1 ............................................... 71

Figure 17: AE \& UT Combination Trial 2 .......................................... 73

Figure 18: MSE plot of Trial 1 for homogeneous data fusion .............................76

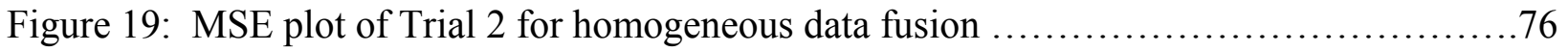

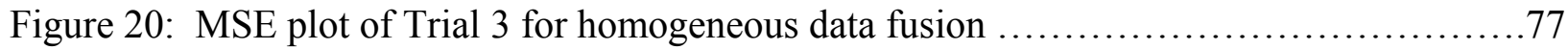

Figure 21: MSE plot of Trial 1 for heterogeneous data fusion $\ldots \ldots \ldots \ldots \ldots \ldots \ldots \ldots \ldots \ldots \ldots . \ldots \ldots$

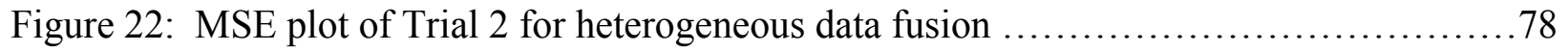




\section{Executive Summary}

The objectives of this research project are:

1. To design sensor data fusion algorithms that can synergistically combine defect related information from heterogeneous sensors used in gas pipeline inspection for reliably and accurately predicting the condition of the pipe-wall.

2. To develop efficient data management techniques for signals obtained during multisensor interrogation of a gas pipeline.

The principal progress during this reporting period involved Task 3.0-Design and Development of Data Fusion Algorithms; Subtask 3.3 - Development of algorithms for defect sizing.

The principal contributions during this reporting period and the conclusions drawn from the work are listed below:

1. The design and development of data fusion algorithms for the prediction of specific information fusion measures - redundancy and complementarity - geometric transformations in combination with radial basis function networks and Parzen windows density estimation techniques have been used. These techniques allowed for the effective extraction of the information measures for a set of pipe-wall test specimens that were inspected in the laboratory using MFL, UT, thermal imaging and AE.

2. The application of the data fusion algorithms to accurately and confidently predict the varying depth profile of surface-breaking pipe wall defects in a gas transmission pipeline - combinations of UT, MFL, thermal imaging and AE NDE data have been fused to predict defect depths in the range of 0.01 " -0.03 " for pipe-wall specimens of thicknesses $5 / 16 "-1 / 2 "$. The accuracy and confidence of the prediction varies in proportion to the information content of the NDE method used for interrogation - for example, MFL-UT combination show higher levels of accuracy than MFL-Thermal combinations.

3. The demonstration of the algorithms ability to fuse data from multiple homogeneous and heterogeneous sensors - redundant and complementary information related to the location and size of a pipe-wall defect was predicted using homogeneous data combinations that include UT-MFL, UT-thermal imaging and MFL-thermal imaging; the heterogeneous data combination includes UT-AE. The heterogeneous data was preprocessed using a Parzen windows technique to generate an equivalent homogeneous 
data set, before being fused with other homogeneous data. The accuracy of the resulting data fusion is critically dependent on the accuracy of the pre-processing.

Results from this research activity were presented and published in the proceedings of the Review of Progress in Quantitative Nondestructive Evaluation (QNDE) conference in Golden, Colorado, July 2004 and formed the basis of Mr. Joseph Oagaro's Master's Thesis, published by Rowan University in December 2004 [1]. 


\section{Experimental}

The principal progress during this reporting period involved Task 3.0 - Design and Development of Data Fusion Algorithms. Results from this research activity were presented and published in the proceedings of the Review of Progress in Quantitative Nondestructive Evaluation (QNDE) conference in Golden, Colorado, July 2004 and formed the basis of Mr. Joseph Oagaro's Master's Thesis, published by Rowan University in December 2004 [1].

\section{Task 3.0 - Design and Development Data Fusion Algorithms}

This report describes enhancements to the suite of sensor data fusion algorithms developed for characterizing the pipe-wall condition of gas transmission pipelines when inspected using more than one method of non-destructive testing - details of the algorithms were provided in previous Semi-Annual Technical Reports. The system that is designed aims to synergistically combine information that is present not only in heterogeneous sensors (for example, magnetic, ultrasonic and thermal) but also in heterogeneous data-sets (for example, "hits" obtained during acoustic emission inspection and images obtained from magnetic flux leakage testing). In this reporting period, we concentrate on fusing information from heterogeneous sensors - NDE signatures have been obtained from a test-specimen suite that has been described previous reports. The objective of the data fusion algorithms is to improve the accuracy and reliability of pipeline monitoring by providing the location, size and shape of pipe-wall anomalies.

This report describes research and development activities Subtask 3.3-Development of algorithms for defect sizing.

\section{Subtask 3.3 - Development of algorithms for defect sizing}

This section will be segmented into the homogeneous data fusion section and the heterogeneous data fusion section. Each section will discuss the details behind the data fusion process and how the redundant and complementary information was extracted from the NDE inspection data. Training and testing sets will also be defined for each section followed by the resulting fused data with redundant and complementary information. First, we revisit the definition of redundant and complementary information in fused data. 
It is the goal of the data fusion neural network to interpolate the redundant and complementary information as well as the intensity of the defect region. To perform this operation the neural network must be trained in the difference between redundant and complementary information. Therefore it is necessary to develop a definition that defines redundant and complementary information for multi-sensor NDE data in terms of the defect geometry. Figure 1 illustrates the definition of redundant and complementary information used in the exercise of the data fusion algorithms.
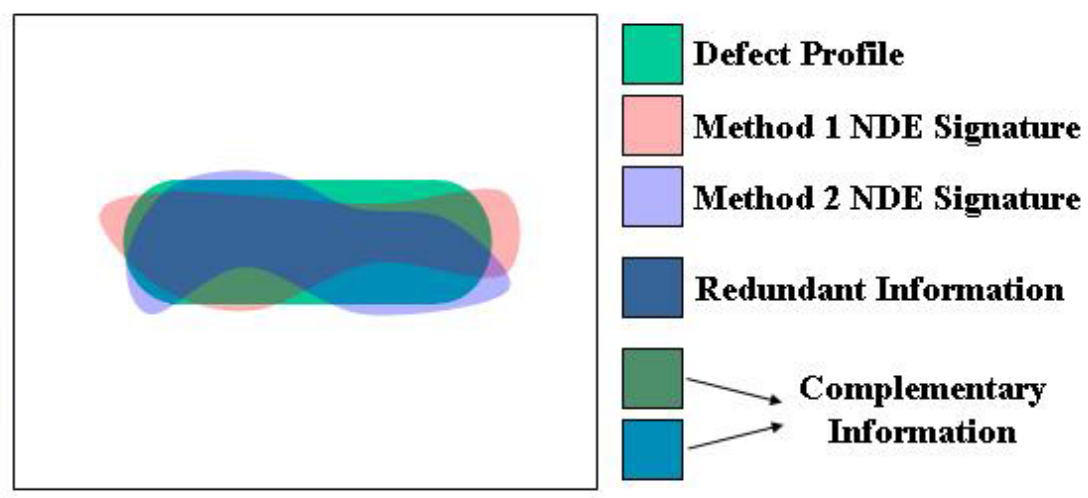

Figure 1: Redundant and complementary data definitions between two NDE signatures.

Pixel gray values are assigned corresponding to the depth of the defect at that location. Complementary information in two NDE images are defined as those distinct pixels in each of the NDE signatures that are present in the defect region, but are not shared between them. Redundant information in two NDE images are defined as those common pixels that are present in both NDE signatures and are also present in the defect region. During the implementation of the data fusion approach, discrete Cosine transforms (DCTs) of the gray level images have been obtained and the 80 highest spectral coefficients have been used as feature vectors that are input to the neural network.

\section{Homogeneous Training and Test Datasets}

The homogeneous data fusion is performed on the NDE inspection data collected from the ultrasound, MFL, and thermal imaging systems. In each test platform the twelve specimens have been scanned twice on two separate instances to provide additional data for training and testing the neural network. The data fusion technique is performed on three different instances between: UT \& MFL, Thermal \& UT, and MFL \& Thermal. Each test sequence includes three separate 
trials that vary the training and test data inputted into the network. The training and test data segmentation for each trial is listed below in Tables 1 through 3.

Table 1: Trial 1 training and test dataset.

\begin{tabular}{|c|c|c|c|}
\hline Specimen \# & Plate thickness (in) & \multicolumn{1}{c|}{ Indication } & Defect Depth (in) \\
\hline $00 a$ & 0.5 & None & N/A \\
\hline $03 a$ & 0.5 & Pitting & 0.0945 \\
\hline $02 a$ & 0.5 & Pitting & 0.198 \\
\hline $01 a$ & 0.5 & Pitting & 0.3005 \\
\hline $10 a$ & 0.375 & None & N/A \\
\hline $13 a$ & 0.375 & Pitting & 0.1105 \\
\hline $12 a$ & 0.375 & Pitting & 0.199 \\
\hline $11 a$ & 0.375 & Pitting & 0.298 \\
\hline $20 a$ & 0.3125 & None & N/A \\
\hline $23 a$ & 0.3125 & Pitting & 0.0995 \\
\hline $22 a$ & 0.3125 & Pitting & 0.1955 \\
\hline $21 a$ & 0.3125 & Pitting & 0.303 \\
\hline $00 b$ & 0.5 & None & N/A \\
\hline $03 b$ & 0.5 & Pitting & 0.0945 \\
\hline $02 b$ & 0.5 & Pitting & 0.198 \\
\hline $01 b$ & 0.5 & Pitting & 0.3005 \\
\hline $10 b$ & 0.375 & None & N/A \\
\hline $13 b$ & 0.375 & Pitting & 0.1105 \\
\hline $12 b$ & 0.375 & Pitting & 0.199 \\
\hline $11 b$ & 0.375 & Pitting & 0.298 \\
\hline $20 b$ & 0.3125 & None & N/A \\
\hline $23 b$ & 0.3125 & Pitting & 0.0995 \\
\hline $22 b$ & 0.3125 & Pitting & 0.1955 \\
\hline $21 b$ & 0.3125 & Pitting & 0.303 \\
\hline & & & \\
\hline & & & \\
\hline & & & \\
\hline
\end{tabular}

Training data

Test data 
Table 2: Trial 2 training and test dataset

\begin{tabular}{|c|c|c|c|}
\hline Specimen \# & Plate thickness (in) & Indication & Defect Depth (in) \\
\hline $00 a$ & 0.5 & None & $N / A$ \\
\hline $03 a$ & 0.5 & Pitting & 0.0945 \\
\hline $02 a$ & 0.5 & Pitting & 0.198 \\
\hline $01 a$ & 0.5 & Pitting & 0.3005 \\
\hline $10 a$ & 0.375 & None & $\mathrm{N} / \mathrm{A}$ \\
\hline $13 a$ & 0.375 & Pitting & 0.1105 \\
\hline $12 a$ & 0.375 & Pitting & 0.199 \\
\hline $11 a$ & 0.375 & Pitting & 0.298 \\
\hline $20 a$ & 0.3125 & None & $\mathrm{N} / \mathrm{A}$ \\
\hline $23 a$ & 0.3125 & Pitting & 0.0995 \\
\hline $22 a$ & 0.3125 & Pitting & 0.1955 \\
\hline $21 a$ & 0.3125 & Pitting & 0.303 \\
\hline $00 \mathrm{~b}$ & 0.5 & None & N/A \\
\hline $03 b$ & 0.5 & Pitting & 0.0945 \\
\hline $02 \mathrm{~b}$ & 0.5 & Pitting & 0.198 \\
\hline $01 \mathrm{~b}$ & 0.5 & Pitting & 0.3005 \\
\hline $10 \mathrm{~b}$ & 0.375 & None & $\mathrm{N} / \mathrm{A}$ \\
\hline $13 b$ & 0.375 & Pitting & 0.1105 \\
\hline $12 \mathrm{~b}$ & 0.375 & Pitting & 0.199 \\
\hline $11 \mathrm{~b}$ & 0.375 & Pitting & 0.298 \\
\hline $20 \mathrm{~b}$ & 0.3125 & None & $\mathrm{N} / \mathrm{A}$ \\
\hline $23 b$ & 0.3125 & Pitting & 0.0995 \\
\hline $22 \mathrm{~b}$ & 0.3125 & Pitting & 0.1955 \\
\hline $21 b$ & 0.3125 & Pitting & 0.303 \\
\hline
\end{tabular}


Table 3: Trial 3 training and test dataset

\begin{tabular}{|c|c|c|c|}
\hline Specimen \# & Plate thickness (in) & Indication & Defect Depth (in) \\
\hline $00 a$ & 0.5 & None & N/A \\
\hline $03 a$ & 0.5 & Pitting & 0.0945 \\
\hline $02 a$ & 0.5 & Pitting & 0.198 \\
\hline $01 a$ & 0.5 & Pitting & 0.3005 \\
\hline $10 a$ & 0.375 & None & N/A \\
\hline $13 a$ & 0.375 & Pitting & 0.1105 \\
\hline $12 a$ & 0.375 & Pitting & 0.199 \\
\hline $11 a$ & 0.375 & Pitting & 0.298 \\
\hline $20 a$ & 0.3125 & None & N/A \\
\hline $23 a$ & 0.3125 & Pitting & 0.0995 \\
\hline $22 a$ & 0.3125 & Pitting & 0.1955 \\
\hline $21 a$ & 0.3125 & Pitting & 0.303 \\
\hline $00 b$ & 0.5 & None & N/A \\
\hline $03 b$ & 0.5 & Pitting & 0.0945 \\
\hline $02 b$ & 0.5 & Pitting & 0.198 \\
\hline $01 b$ & 0.5 & Pitting & 0.3005 \\
\hline $10 b$ & 0.375 & None & N/A \\
\hline $13 b$ & 0.375 & Pitting & 0.1105 \\
\hline $12 b$ & 0.375 & Pitting & 0.199 \\
\hline $11 b$ & 0.375 & Pitting & 0.298 \\
\hline $20 b$ & 0.3125 & None & N/A \\
\hline $23 b$ & 0.3125 & Pitting & 0.0995 \\
\hline $22 b$ & 0.3125 & Pitting & 0.1955 \\
\hline $21 b$ & 0.3125 & Pitting & 0.303 \\
\hline & & & \\
\hline
\end{tabular}

Training data

Test data

The results in Figures 2 through 10 represent the three trials for each of the three combinations of NDE data, with a red border surrounding the subplot figure of test data outputs. Included in each trial are the training data outputs and test data outputs of the neural network seen in the subplots (a) through (x). A red border surrounding the subplot figure designates the test data outputs. The subplots (a) through ( $\mathrm{x}$ ) represent the output for the specimens listed in the order of the trial tables, with the specimens designated for testing shown last, with a red border. For example, subplot (a) represents the training input and output from Specimen 00a, while subplot (b) represents Specimen 03a, and so on. In certain combinations, outlying training data instances were excluded from training and testing, causing there to be fewer subplots in those cases. Each subplot figure is segmented into six images. The first row includes the input data from each of the NDE testing modalities. The second row represents the redundant and complementary output predicted by the algorithm while the third 
row shows the desired redundant and complementary output. All of the results are properly labeled and formatted in this manner.

There is an important difference to note between training data and test data. Training data is used to determine the synaptic weights of the network. Both training inputs and desired outputs must be provided to the network in the training process. In the case of training data seen in subplots in Figures 2-10, the first row, labeled "inputs," are the training inputs. The third row, labeled "desired output" are the outputs that the network is trained to produce when the associated inputs are given to the network. The second row shows the actual output that is produced when a fully trained network is given its own training data inputs as inputs. Test data, on the other hand, is never used to train the network. Therefore, the first row in a test data subplot is the actual input that is given to the network for testing. The second row is the actual (predicted) output of the network for test inputs; these are the test results. The images in the third row (created using the same technique that the training data desired outputs were created with) show, for test data, what the network should be producing as output. These images in the third row provide a means of evaluating the predicted testing data outputs.

Keeping these key differences between training and testing data in mind, it is therefore desired for the redundant desired output image to closely resemble (in theory, be exactly like) the redundant output. Similarly, the complementary desired output image must resemble the complementary output, as closely as possible. This is true for all subplots, for both training and test instances. In training instances, close similarity illustrates that the synaptic weights of the network are optimal and in test instances, close similarity illustrates that the network is able to interpolate for new instances of input data. 
Trial 1: UT \& MFL Results

\section{Ultrasound \& MFL Data Fusion Results}

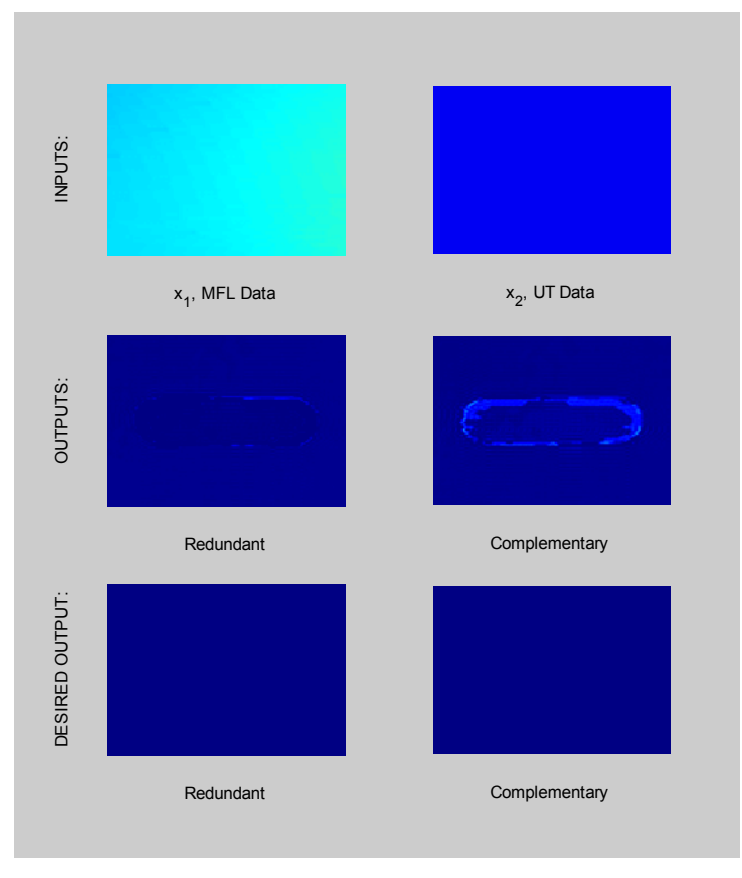

(a) Specimen 00a

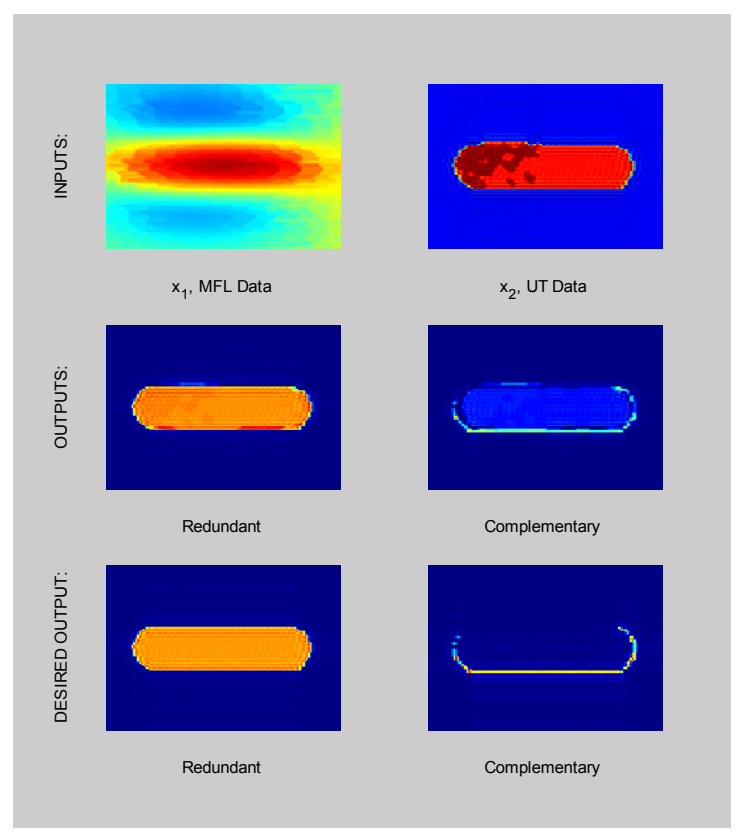

(c) Specimen 02a

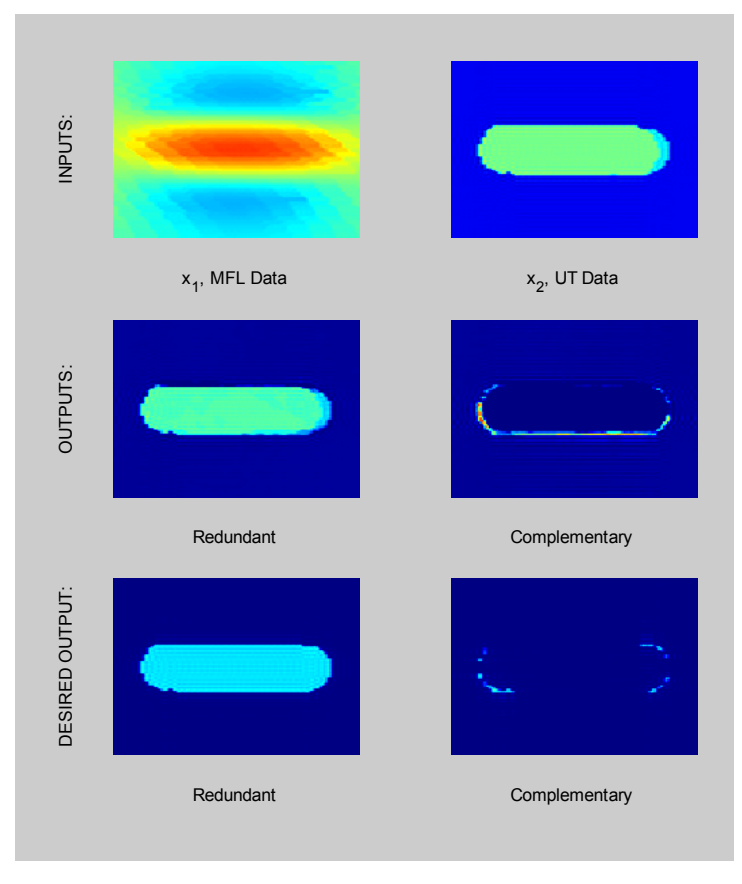

(b) Specimen 03a

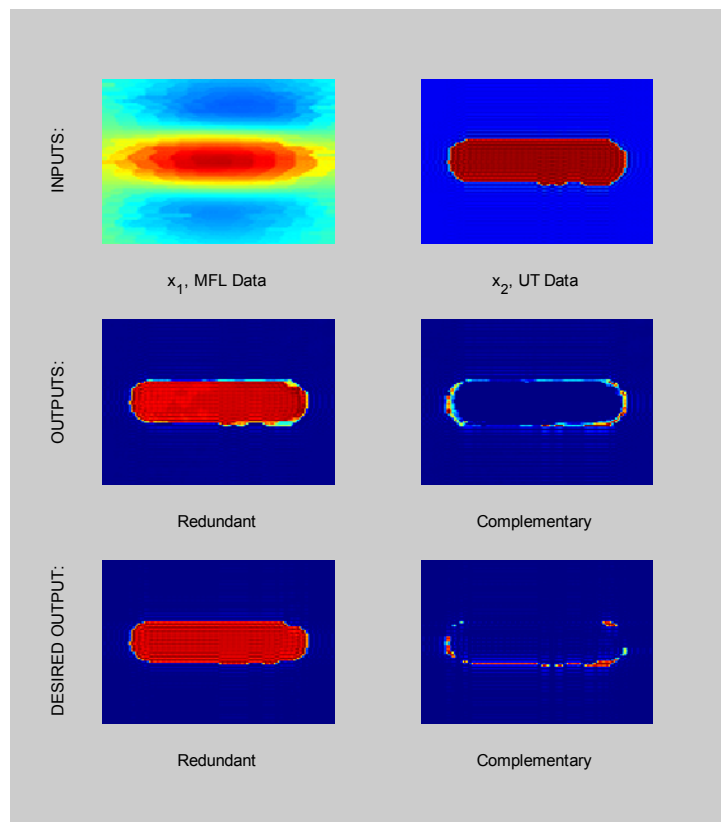

(d) Specimen 01a 
Trial 1: UT \& MFL Results (cont.)

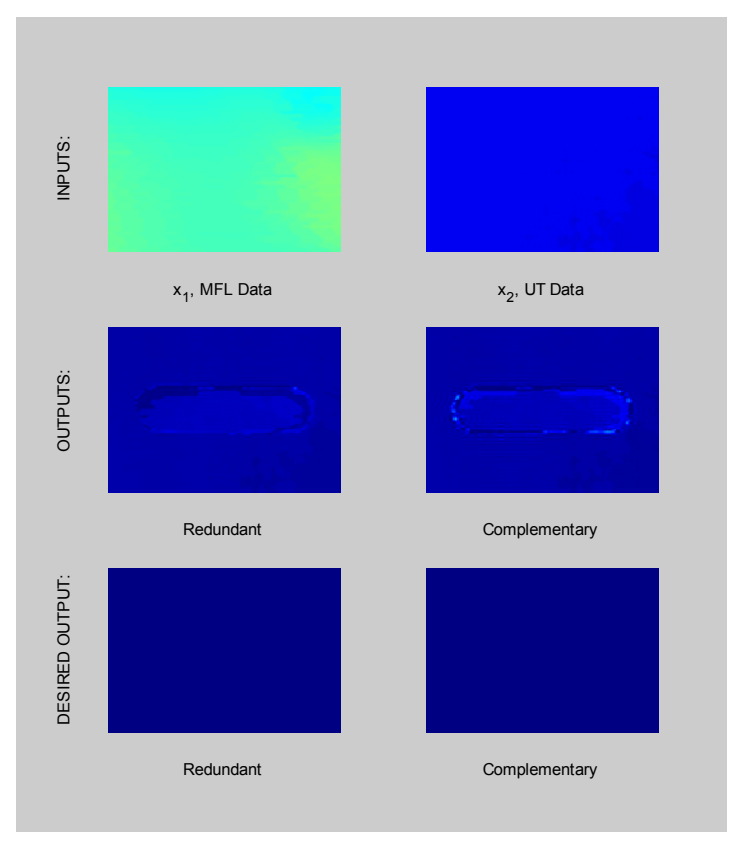

(e) Specimen 10a
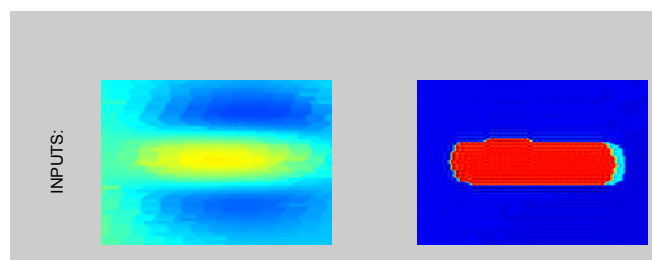

$\mathrm{x}_{1}$, MFL Data

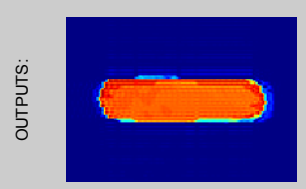

Redundant

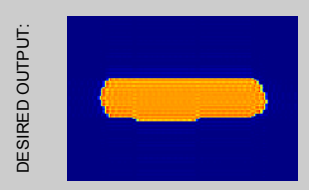

Redundant $\mathrm{x}_{2}$, UT Data

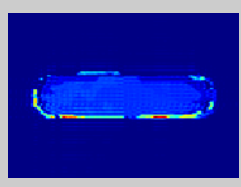

Complementary

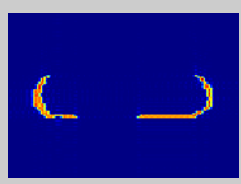

Complementary

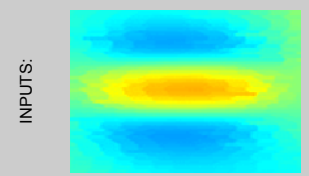

$\mathrm{x}_{1}$, MFL Data

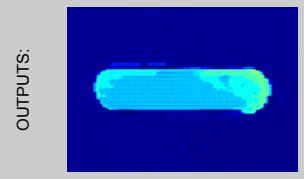

Redundant

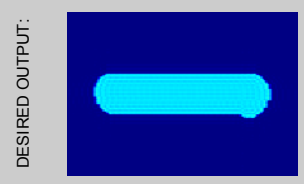

Redundant

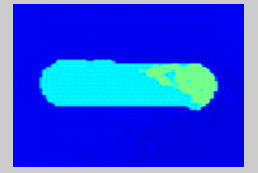

$\mathrm{x}_{2}$, UT Data

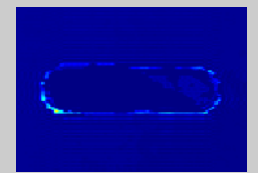

Complementary

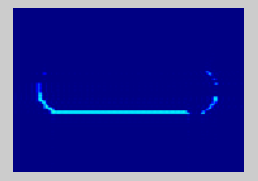

Complementary

(f) Specimen 13a

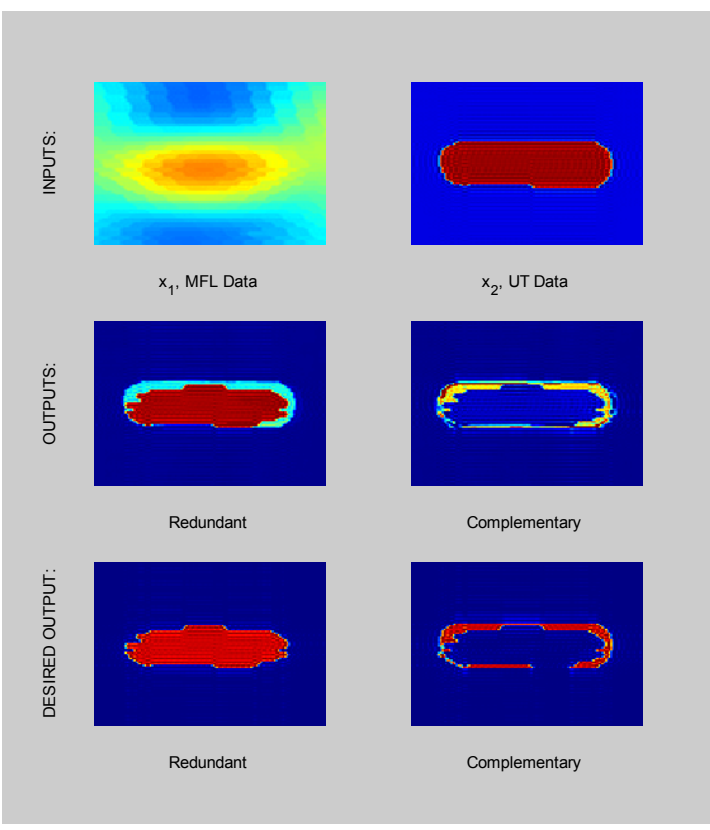

(h) Specimen 11a 
Trial 1: UT \& MFL Results (cont.)

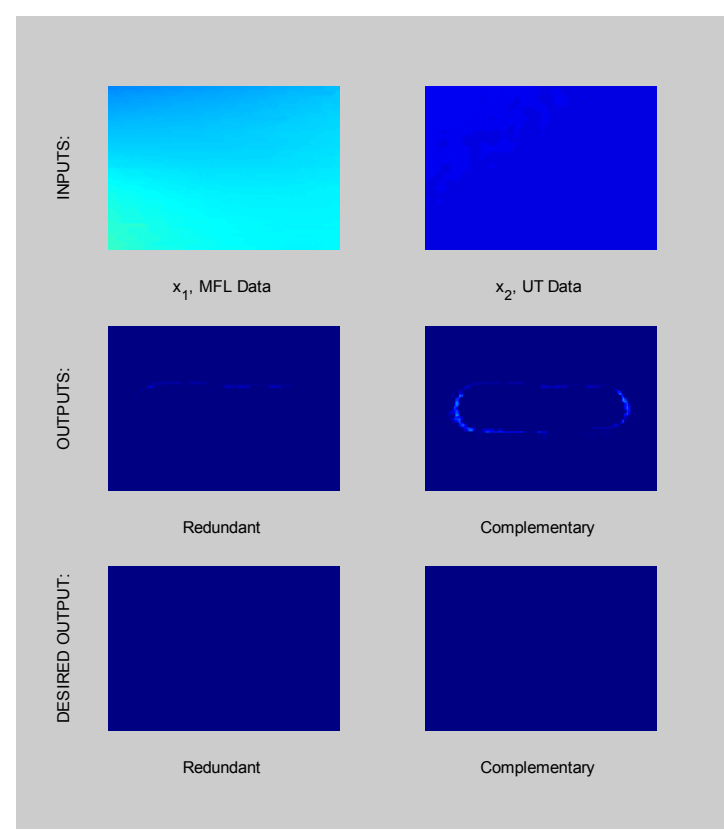

(i) Specimen 20a

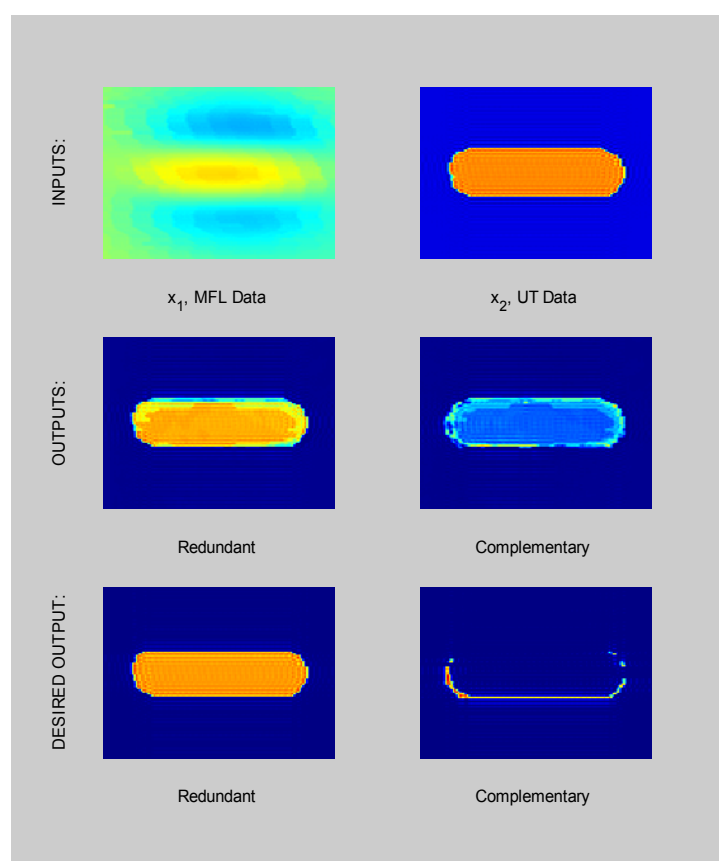

(k) Specimen 22a

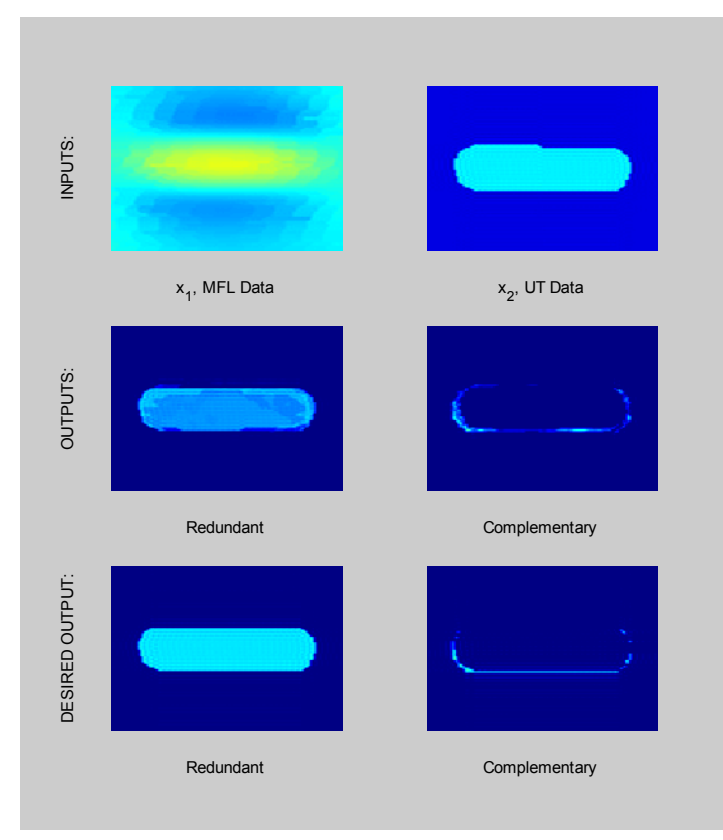

(j) Specimen 23a

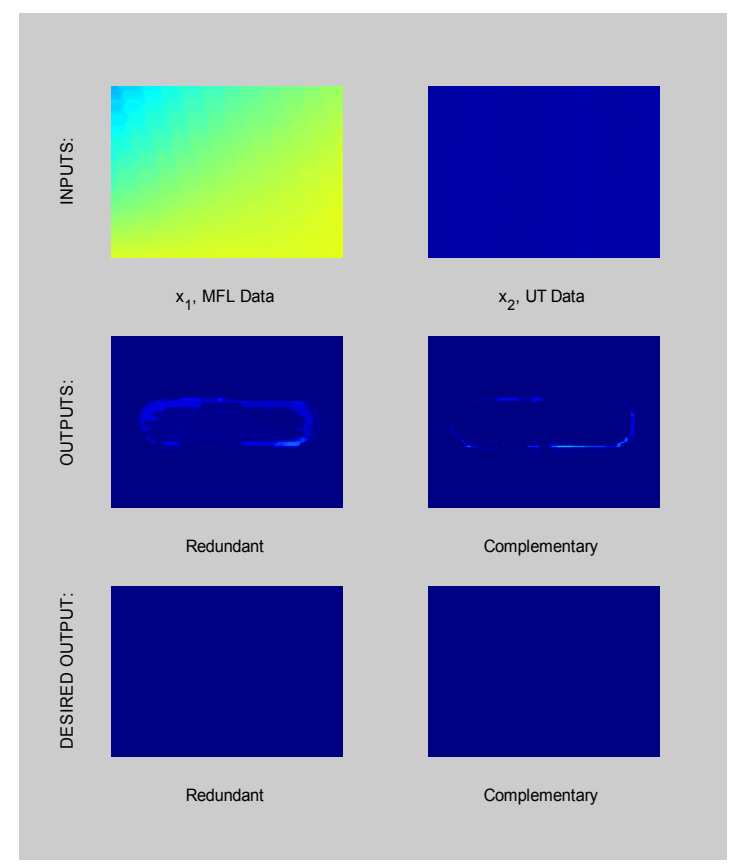

(l) Specimen 00b 
Trial 1: UT \& MFL Results (cont.)

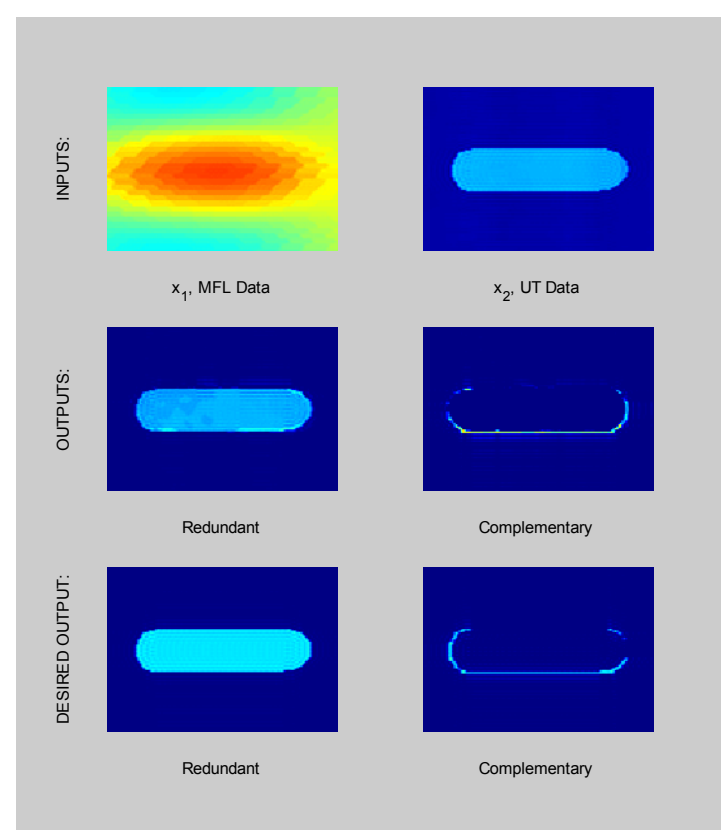

(m) Specimen 03b

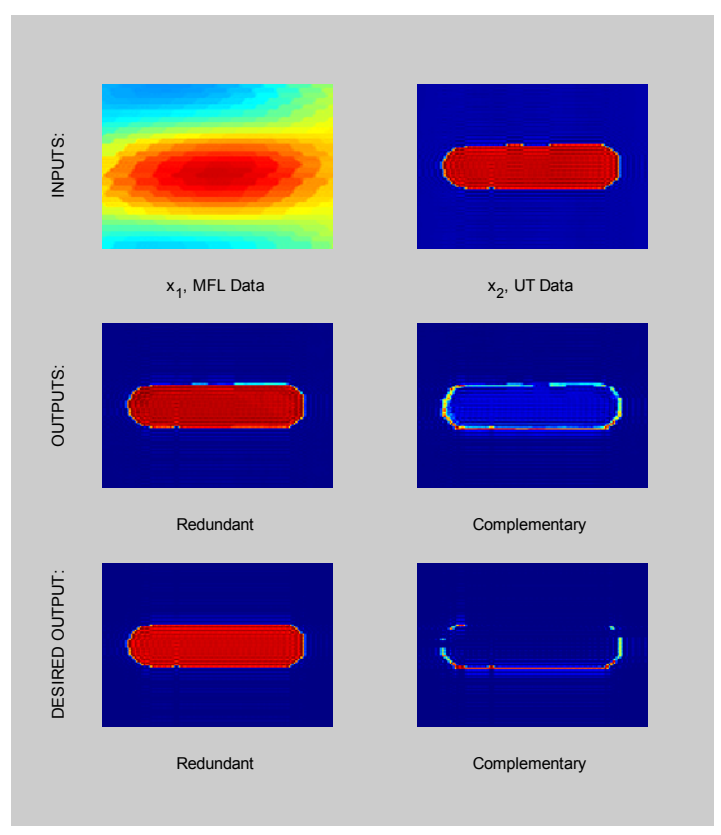

(o) Specimen 01b

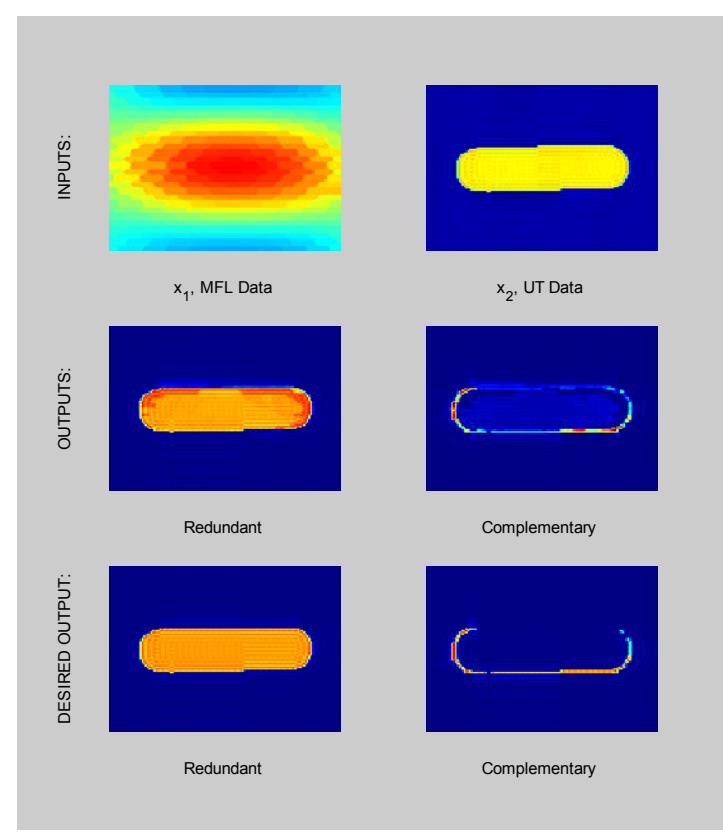

(n) Specimen 02b

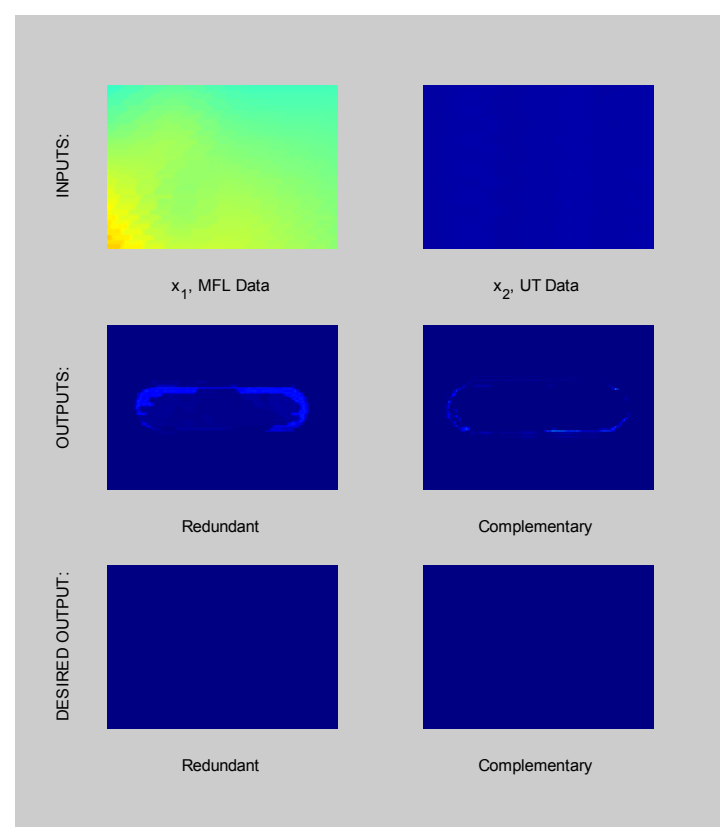

(p) Specimen 10b 
Trial 1: UT \& MFL Results (cont.)

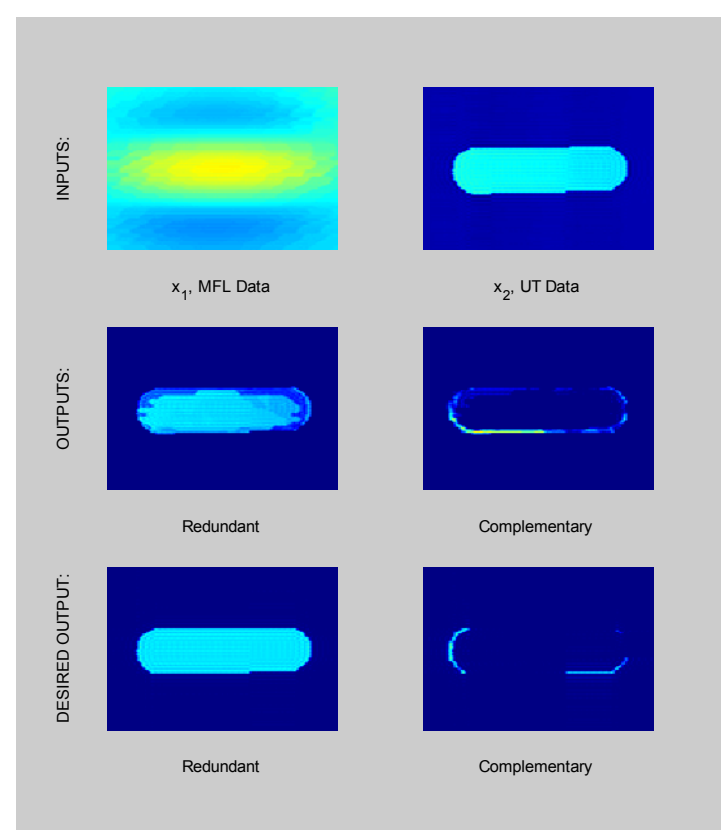

(q) Specimen 13b

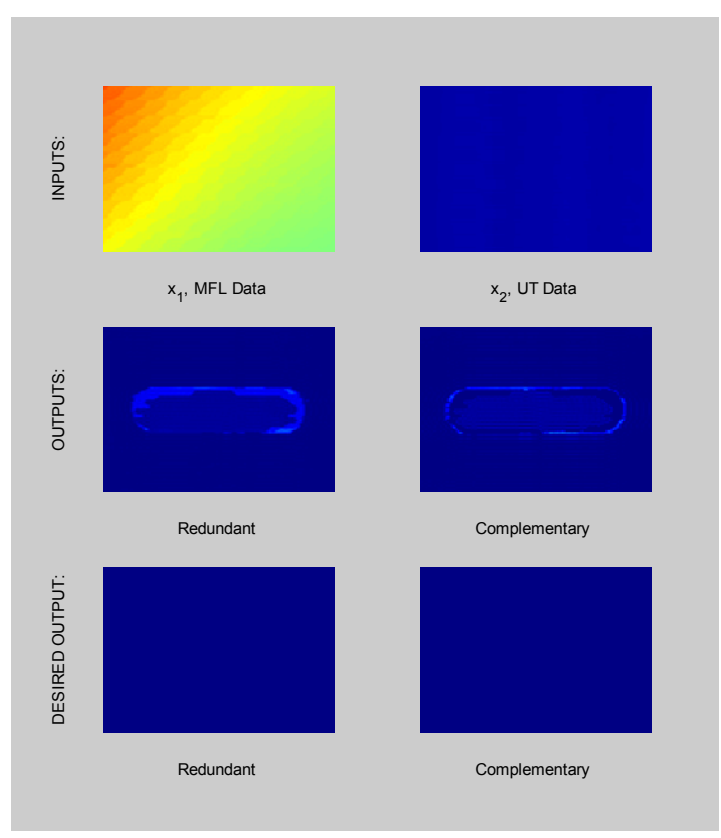

(s) Specimen 20b

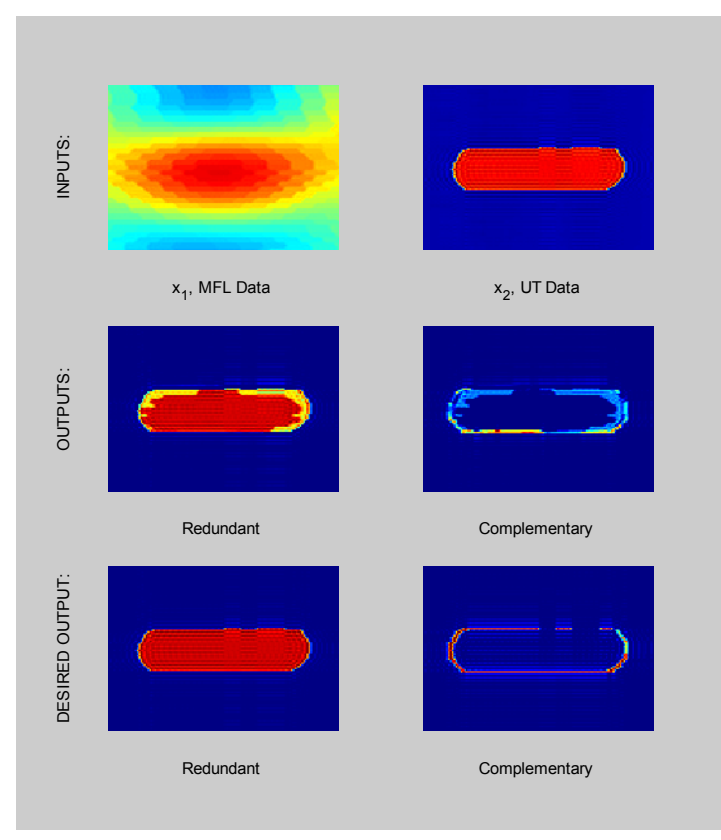

(r) Specimen 11b

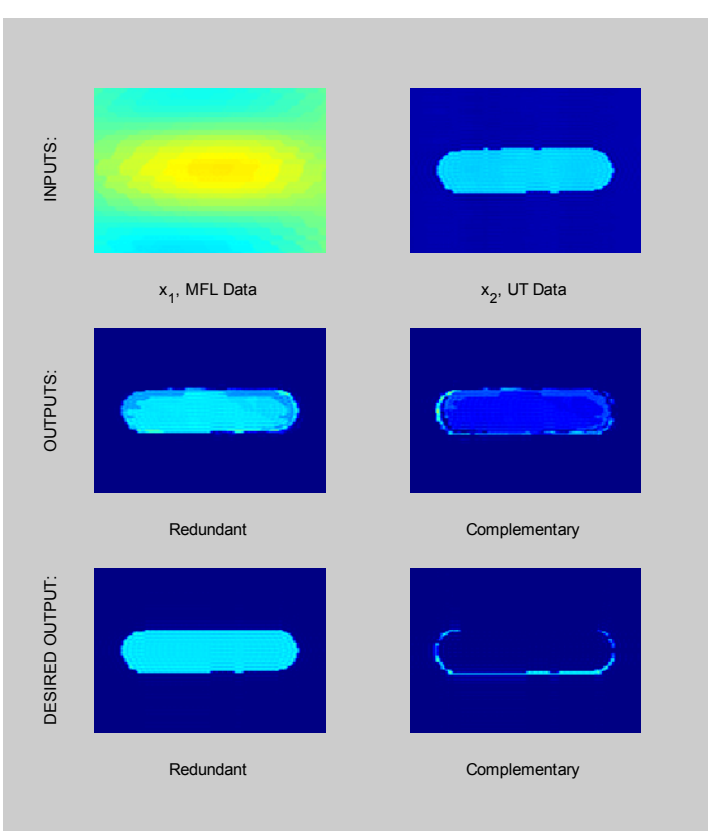

(t) Specimen 23b 
Trial 1: UT \& MFL Results (cont.)

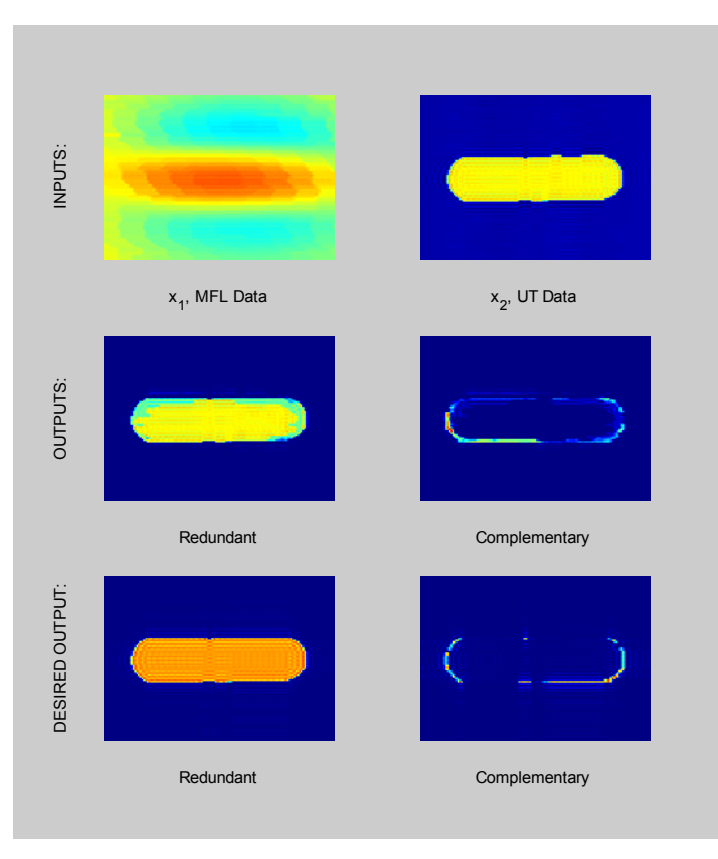

(u) Specimen 22b

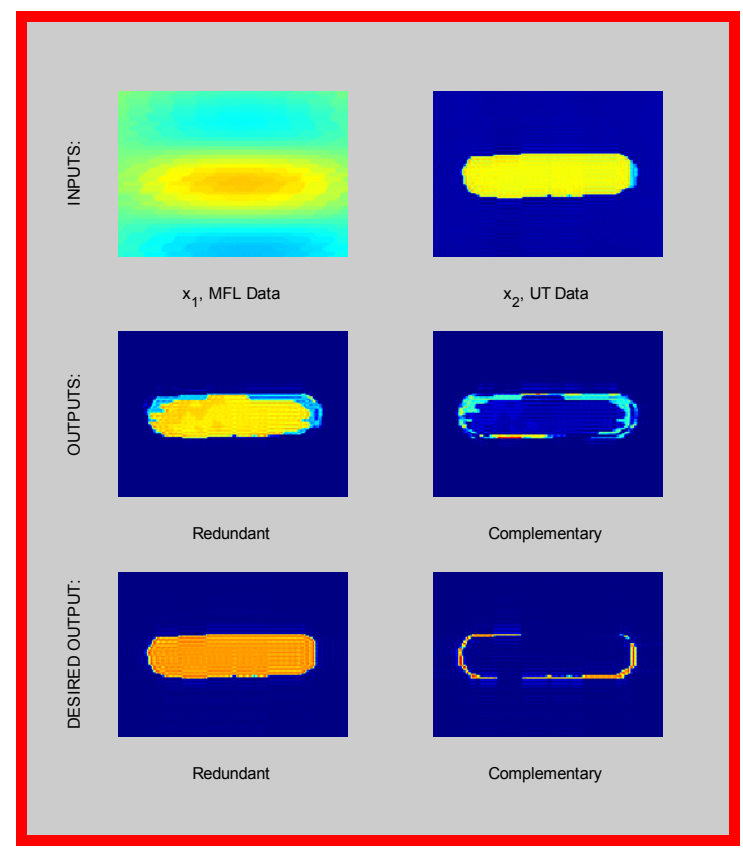

(v) Specimen 01a

Figure 2: UT \& MFL Combination Trial 1

Training Data: (a) - (u); Test Data: (v)

Trial 2: UT \& MFL Results

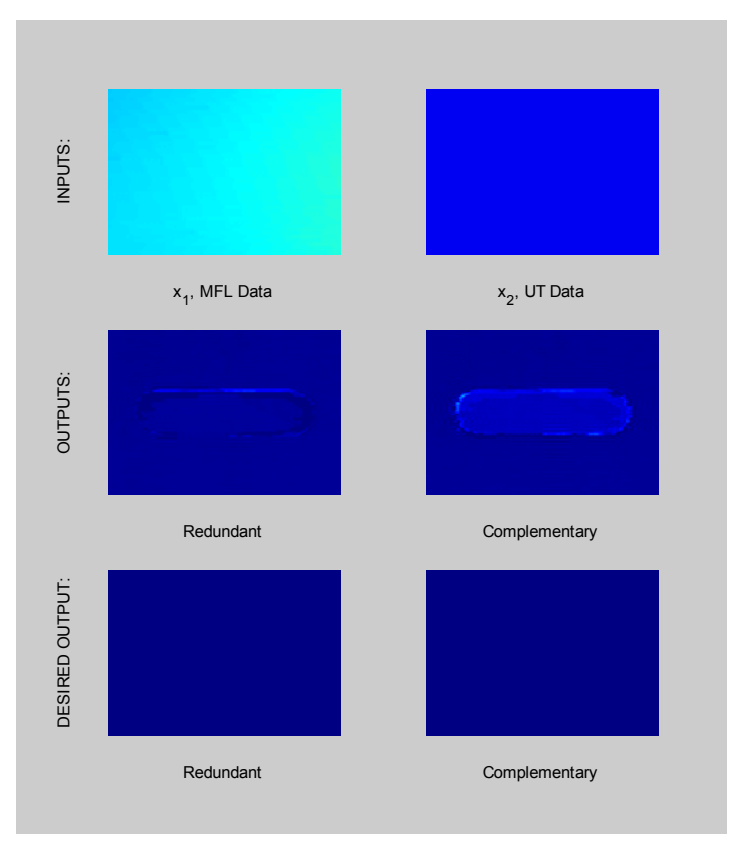

(a) Specimen 00a

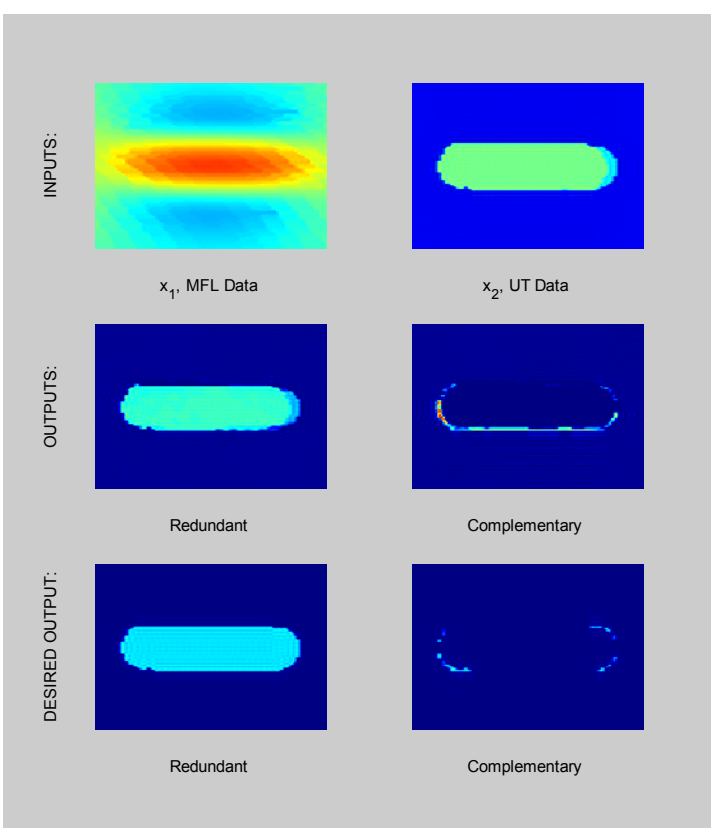

(b) Specimen 03a 
Trial 2: UT \& MFL Results (cont.)

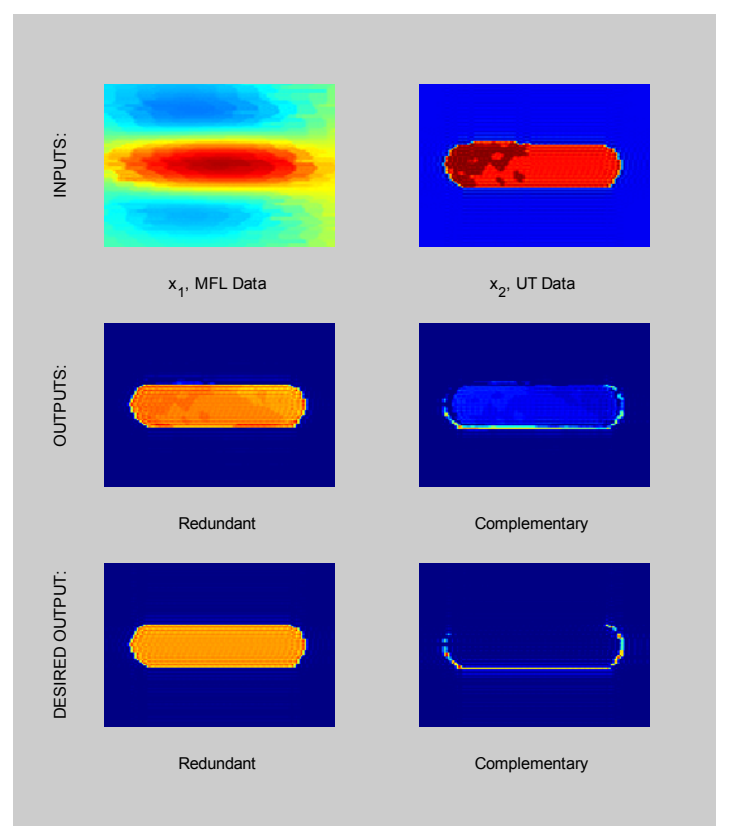

(c) Specimen 02a

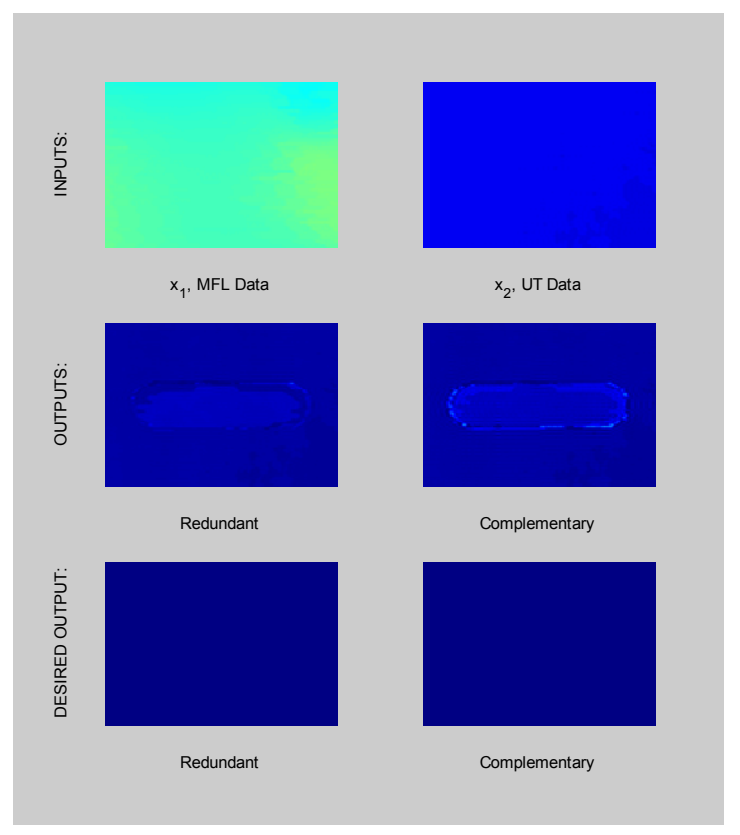

(e) Specimen 10a

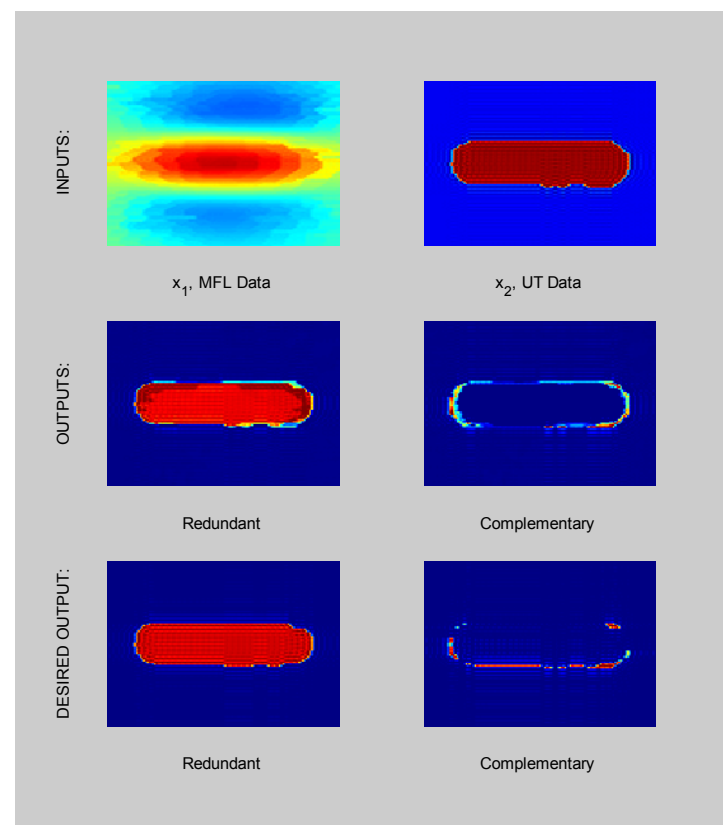

(d) Specimen 01a

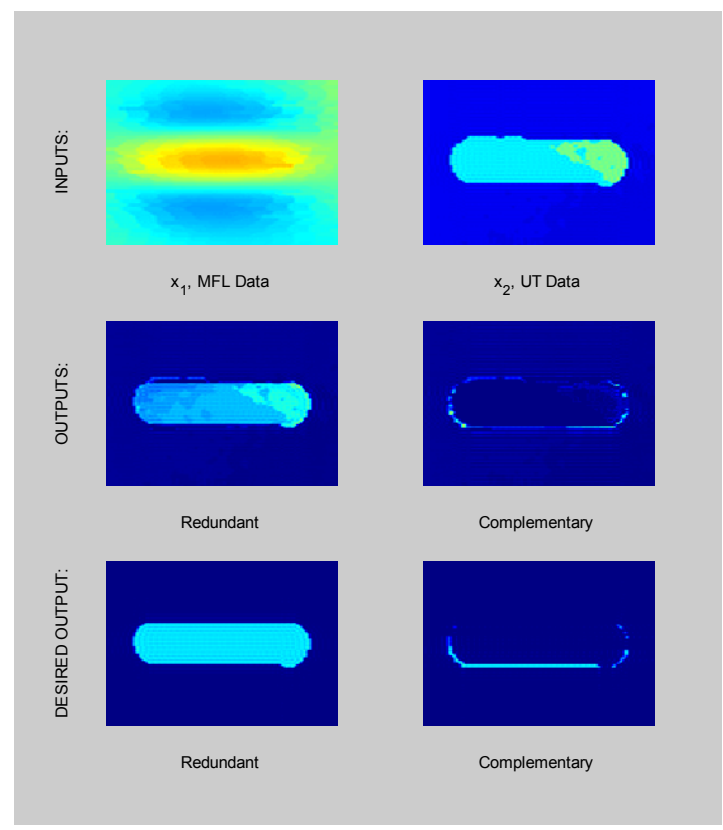

(f) Specimen 13a 
Trial 2: UT \& MFL Results (cont.)

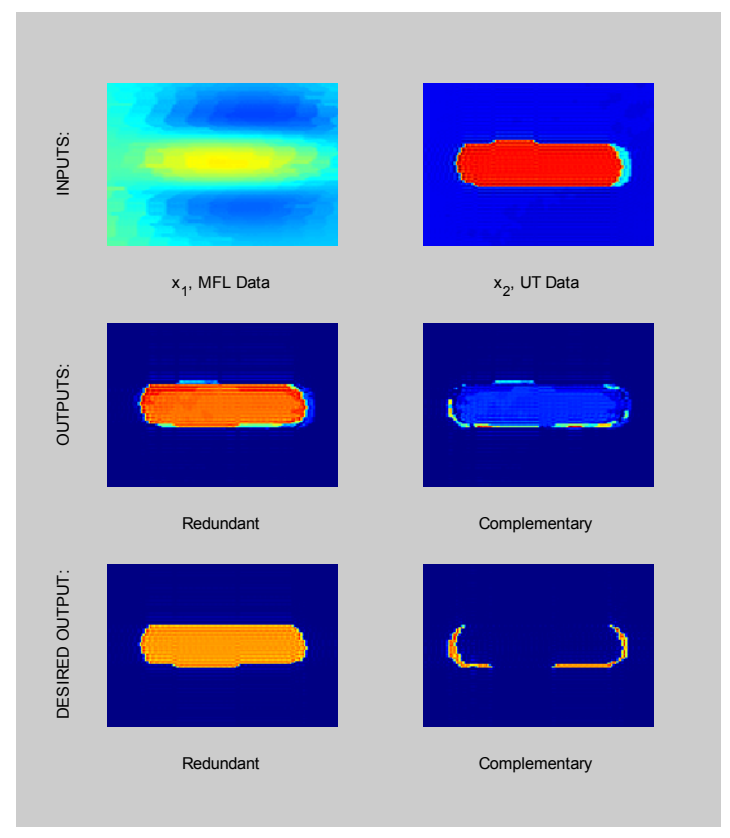

(g) Specimen 12a

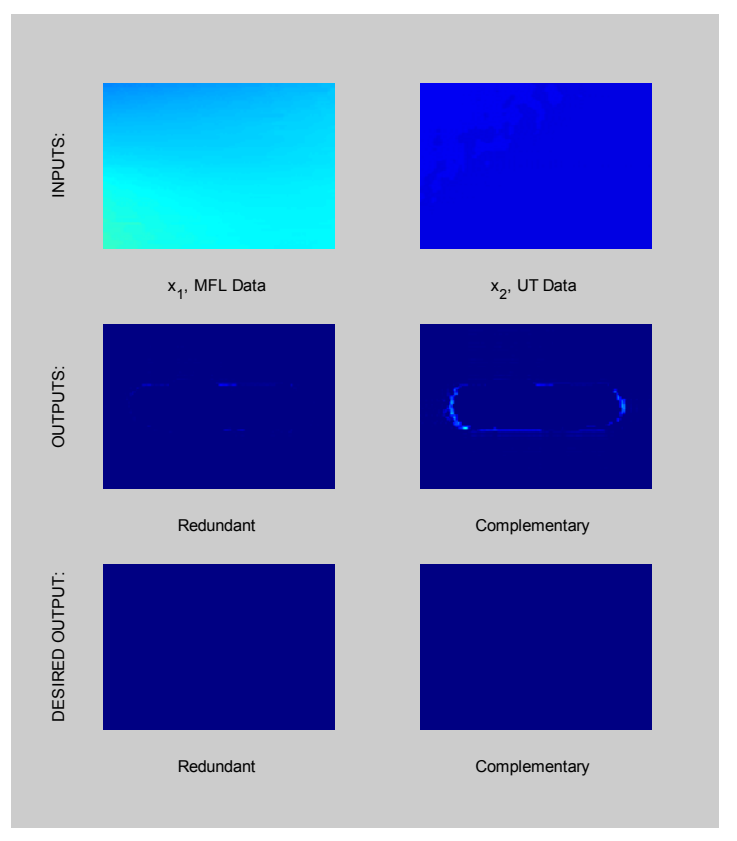

(i) Specimen 20a

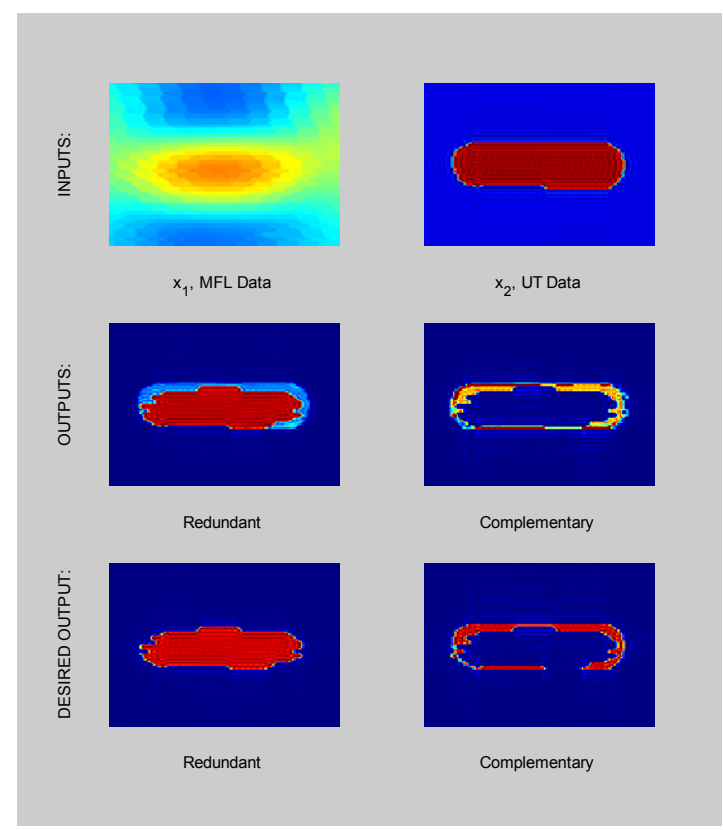

(h) Specimen 11a

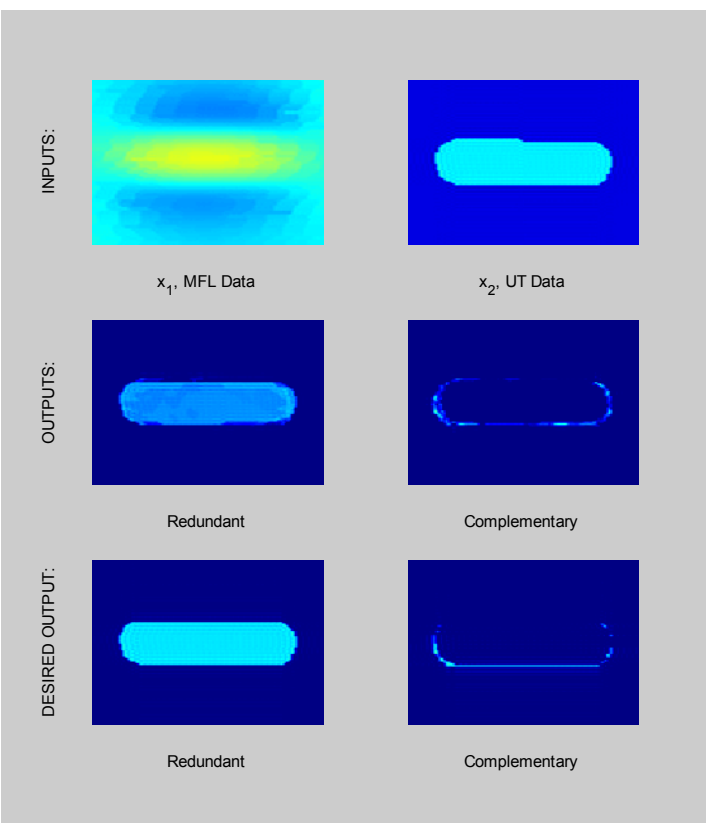

(j) Specimen 23a 
Trial 2: UT \& MFL Results (cont.)

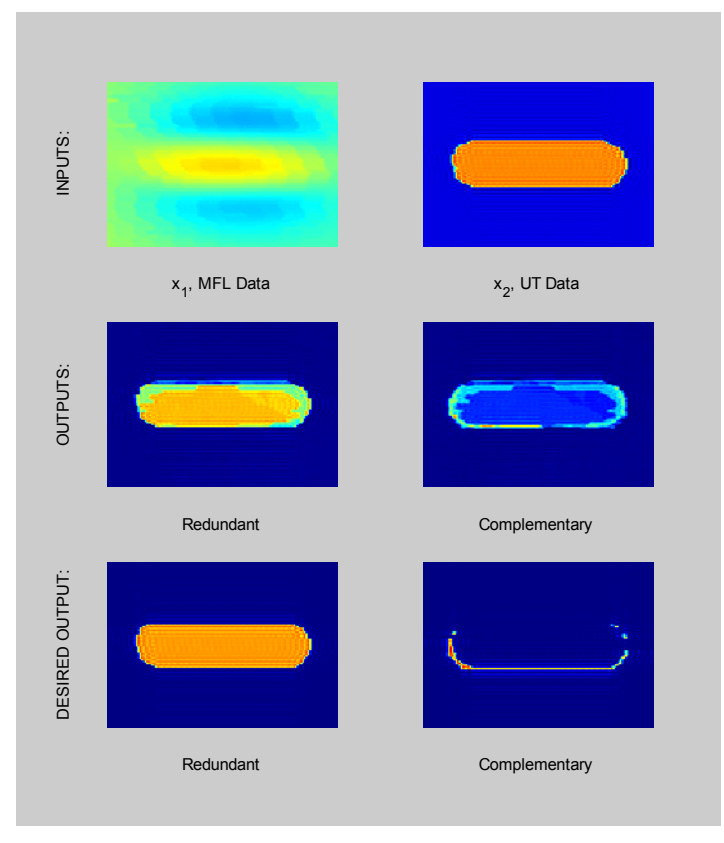

(k) Specimen 22a

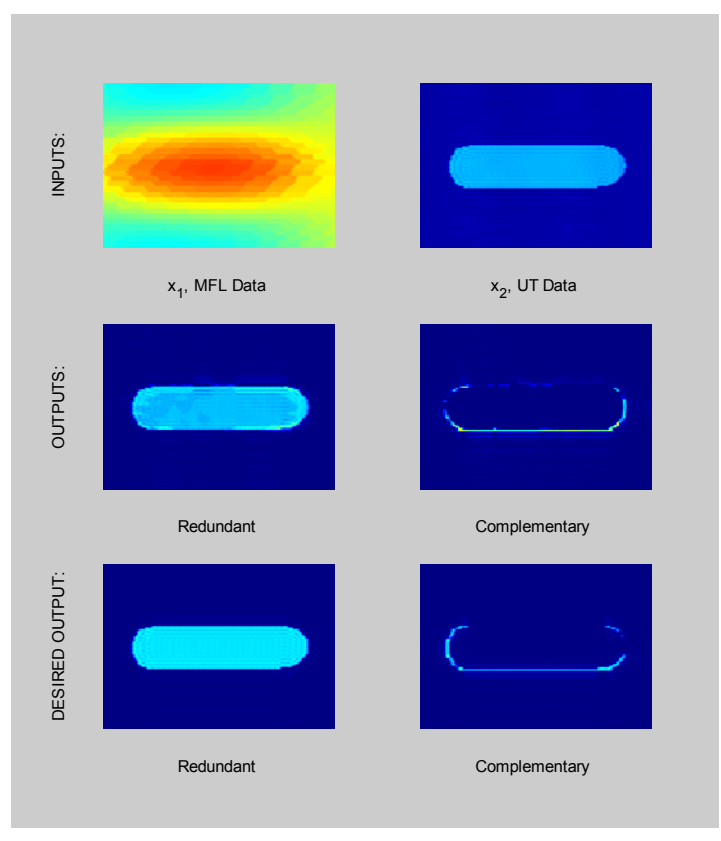

(m) Specimen 03b

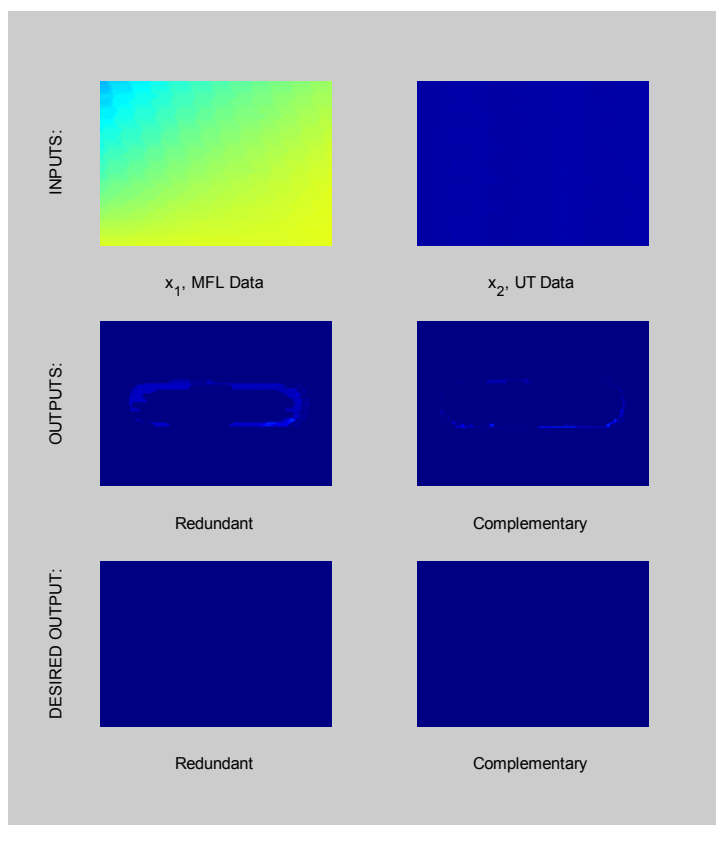

(l) Specimen 00b

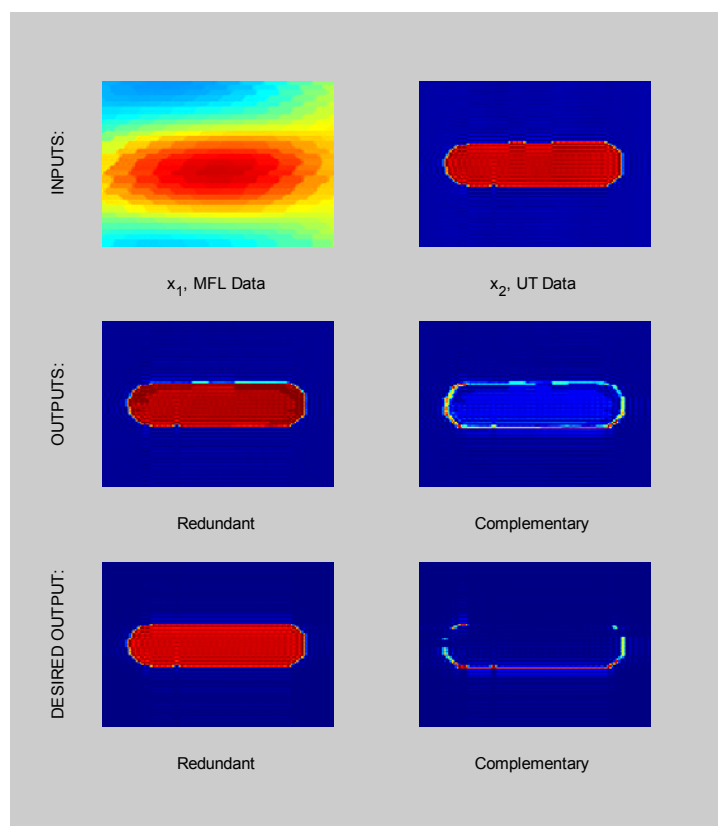

(n) Specimen 01b 
Trial 2: UT \& MFL Results (cont.)

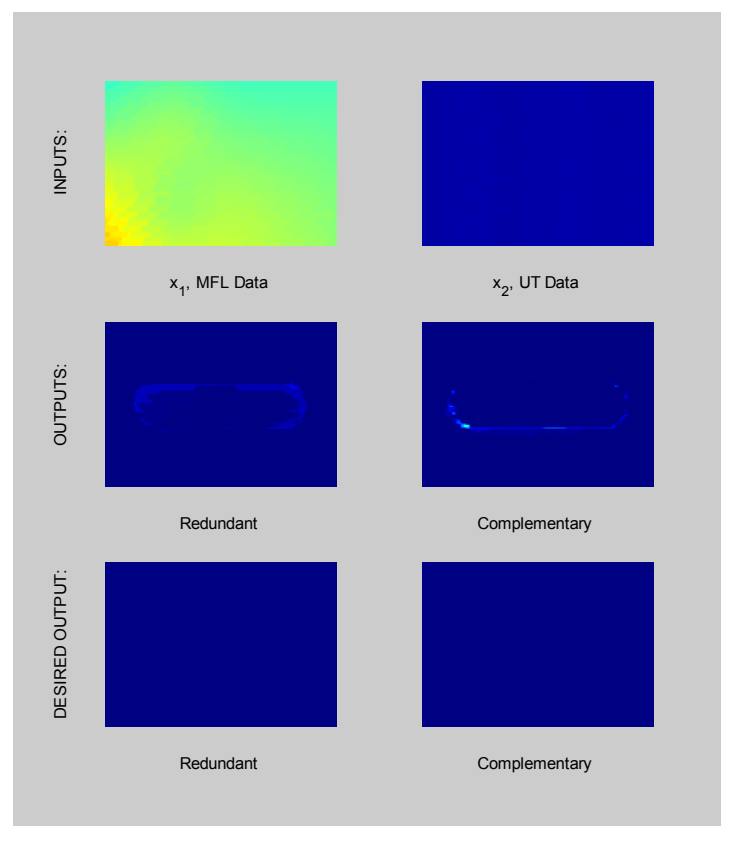

(o) Specimen 10b

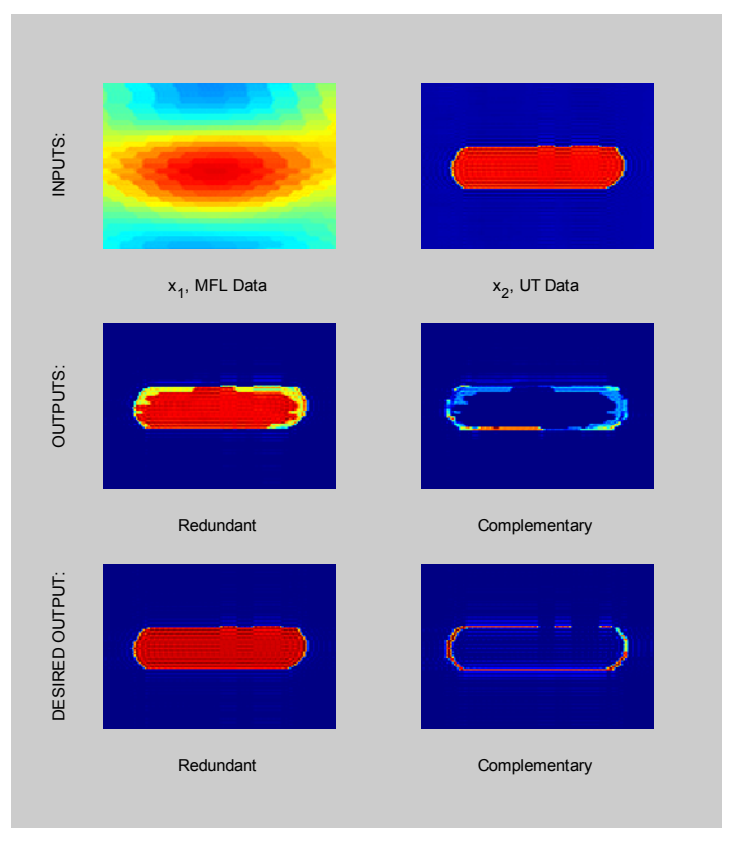

(q) Specimen 11b

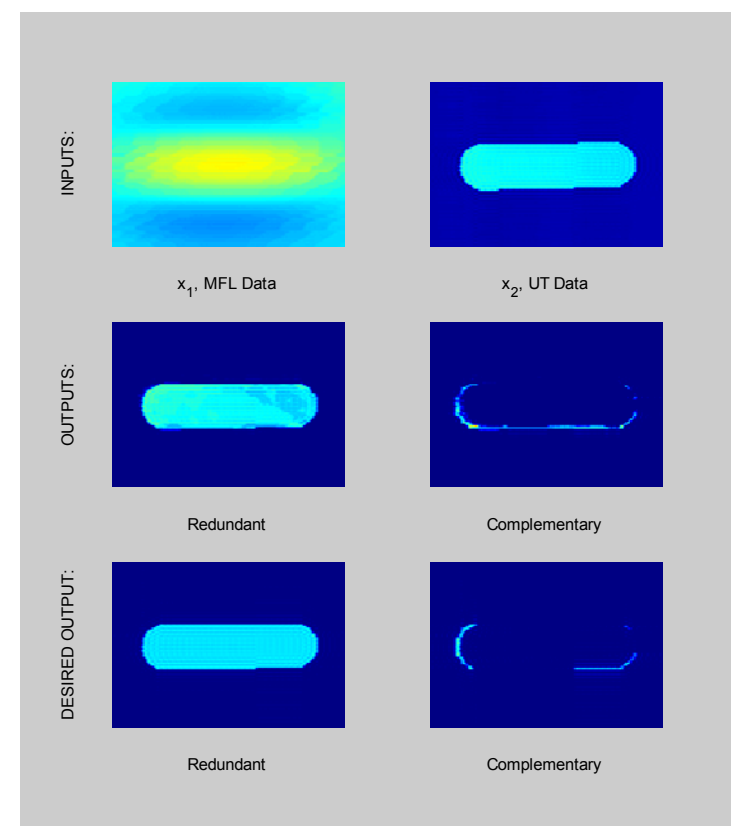

(p) Specimen 13b

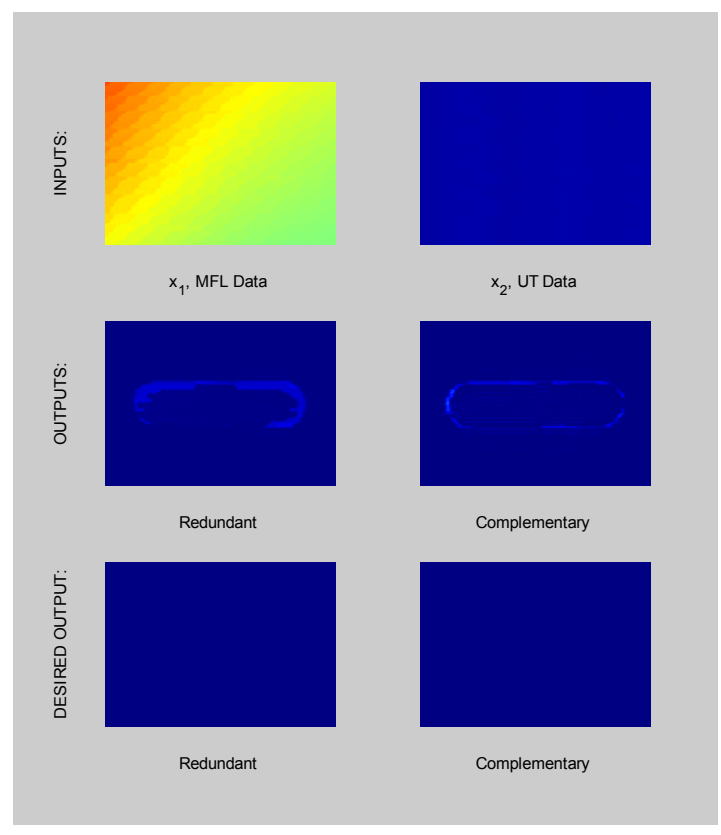

(r) Specimen 20b 
Trial 2: UT \& MFL Results (cont.)

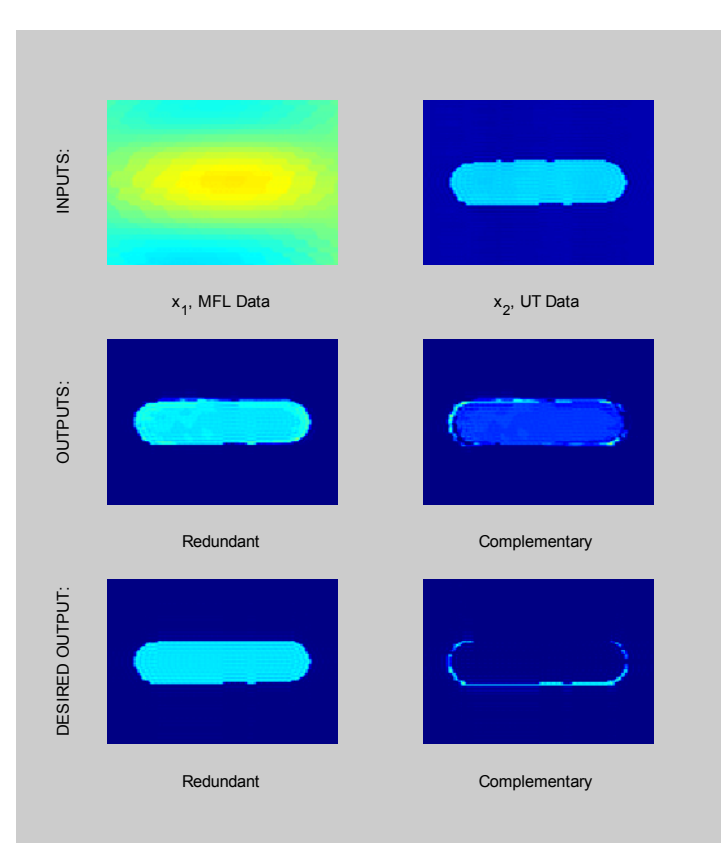

(s) Specimen 23b

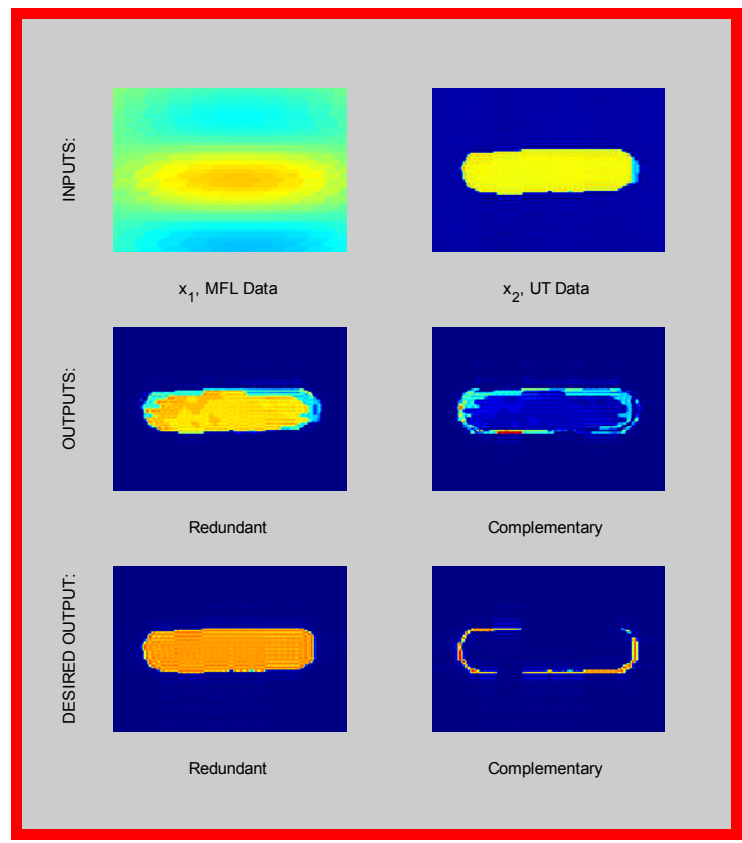

(u) Specimen 12b

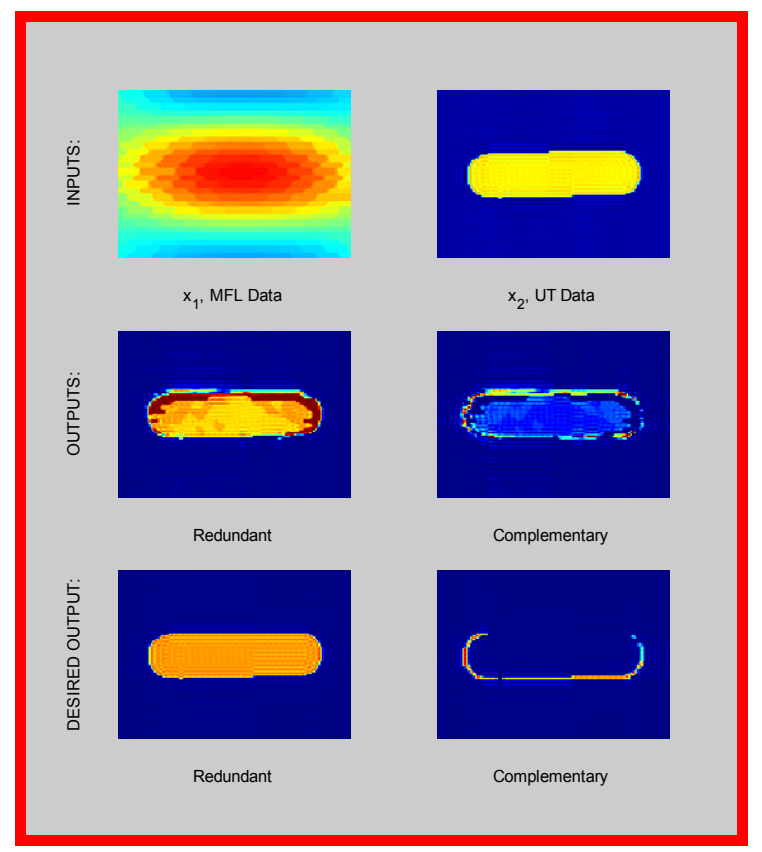

(t) Specimen 02b

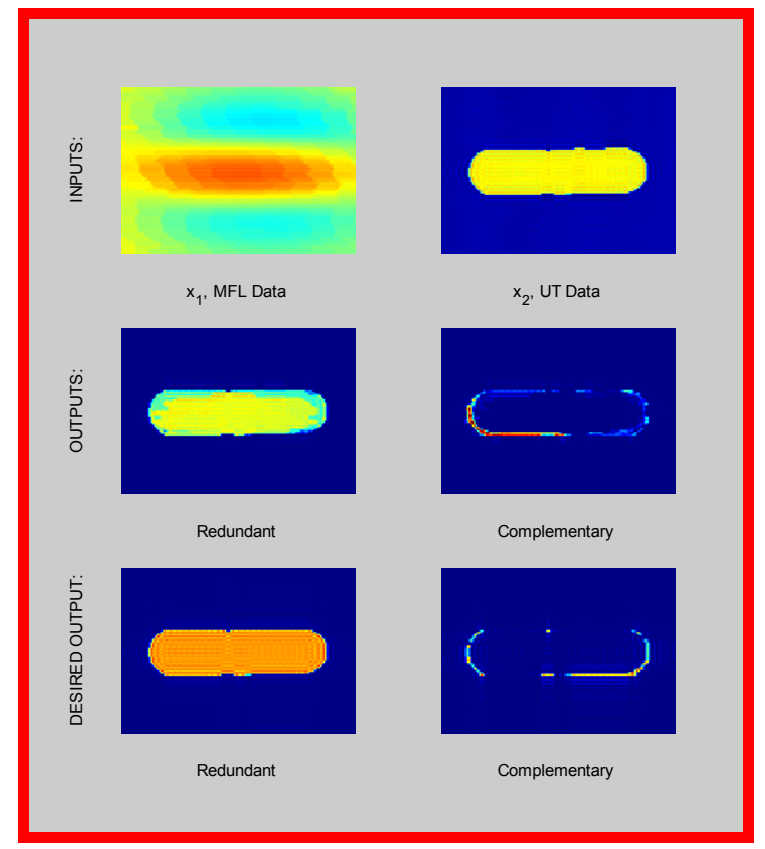

(v) Specimen 22b

Figure 3: UT \& MFL combination Trial 2.

Training Data: (a) - (s); Test Data: (t), (u), (v)

Trial 3: UT \& MFL Results 


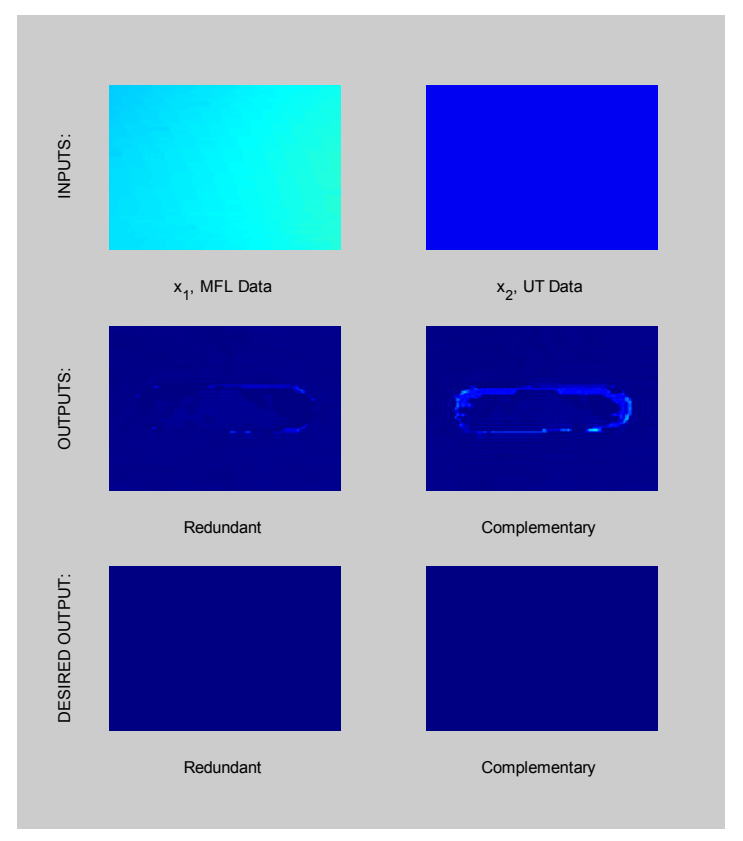

(a) Specimen 00a

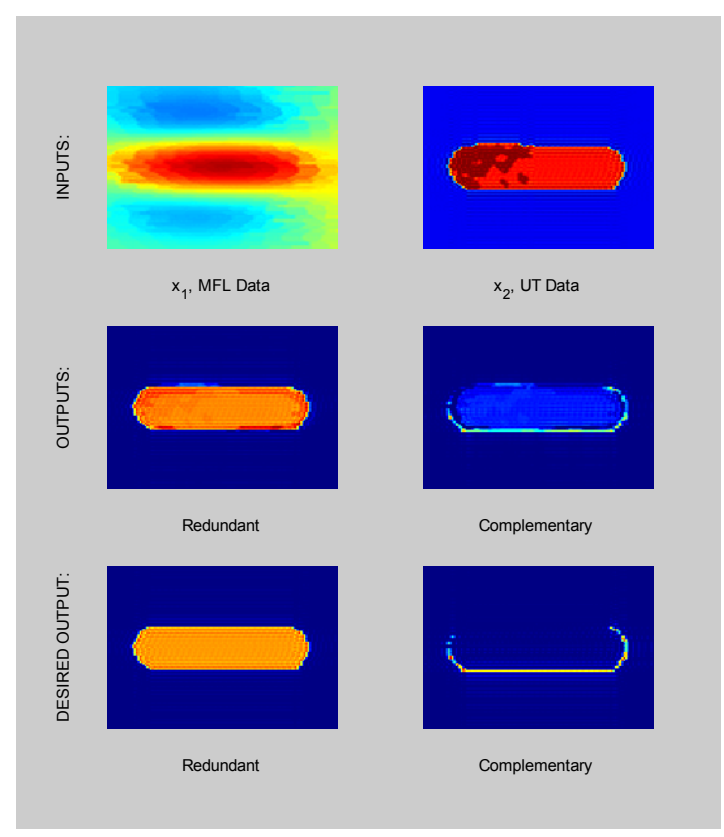

(c) Specimen 02a

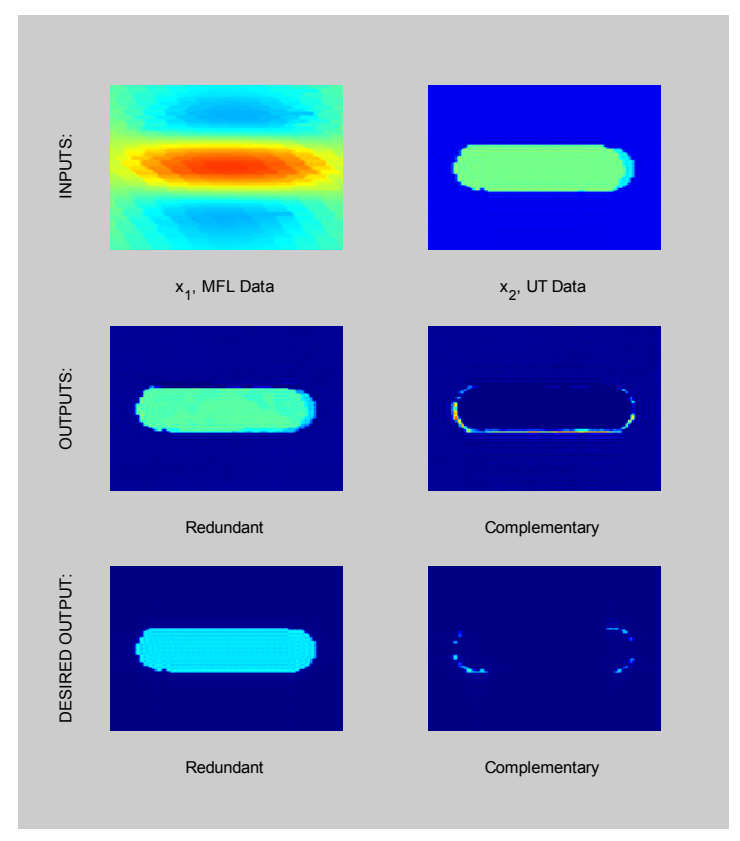

(b) Specimen 03a

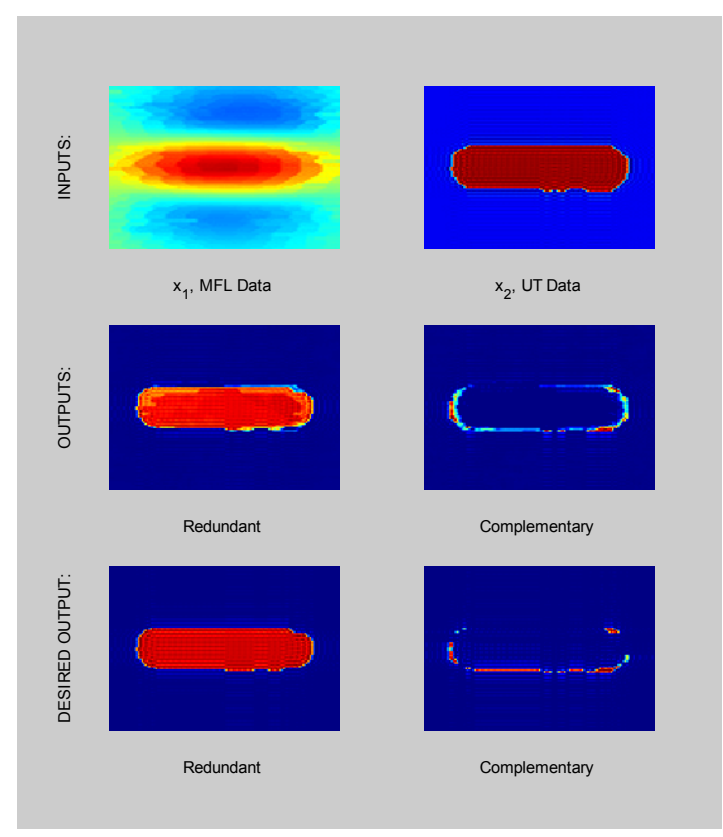

(d) Specimen 01a 
Trial 3: UT \& MFL Results (cont.)

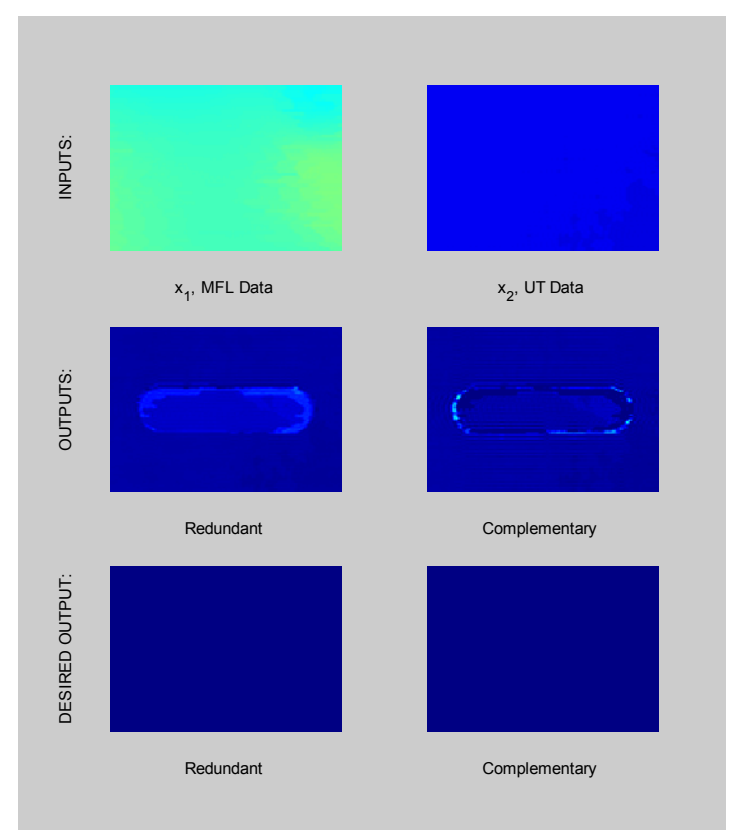

(e) Specimen 10a

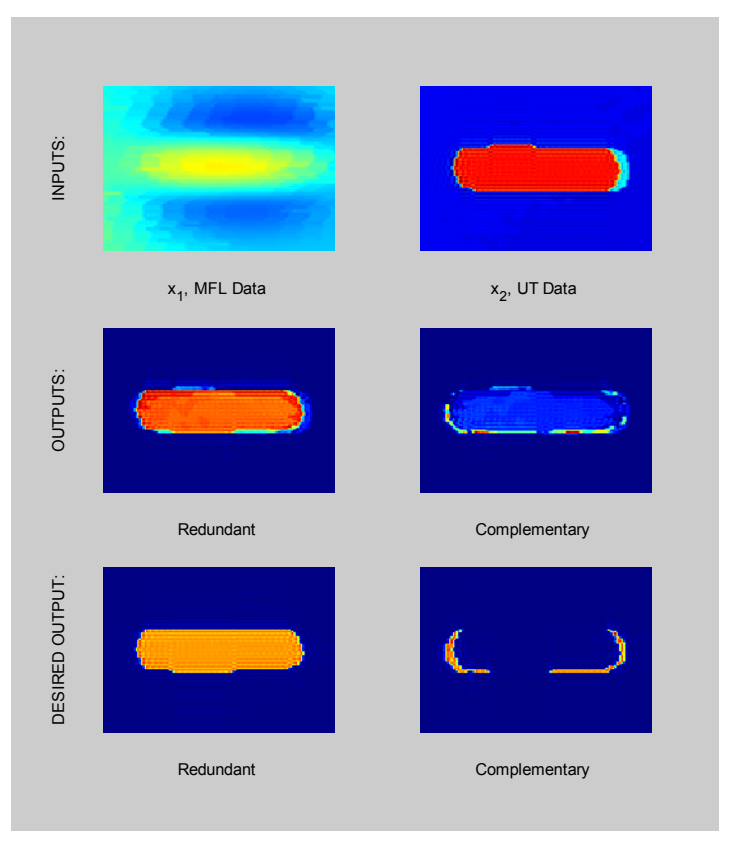

(g) Specimen 12a

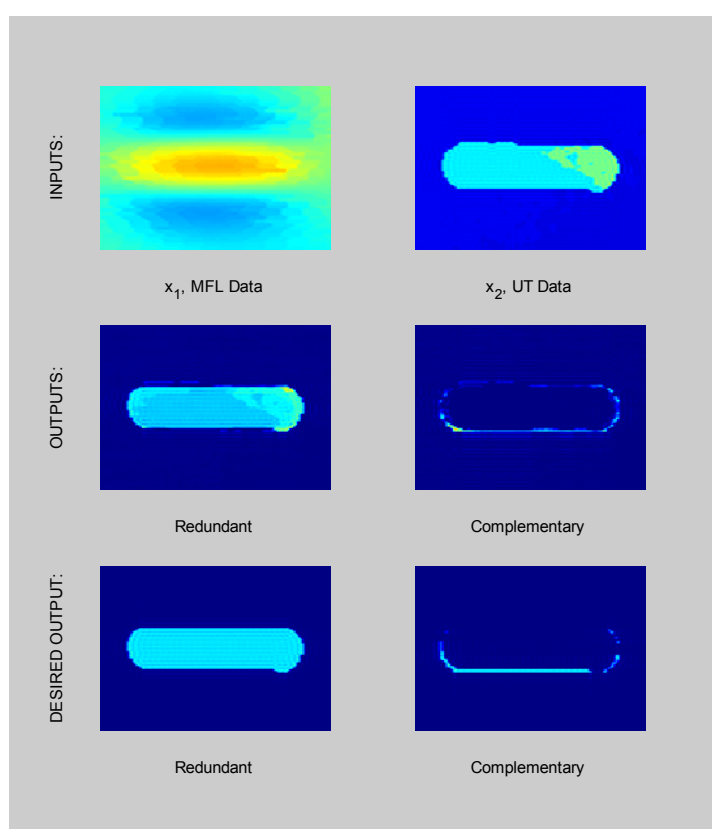

(f) Specimen 13a

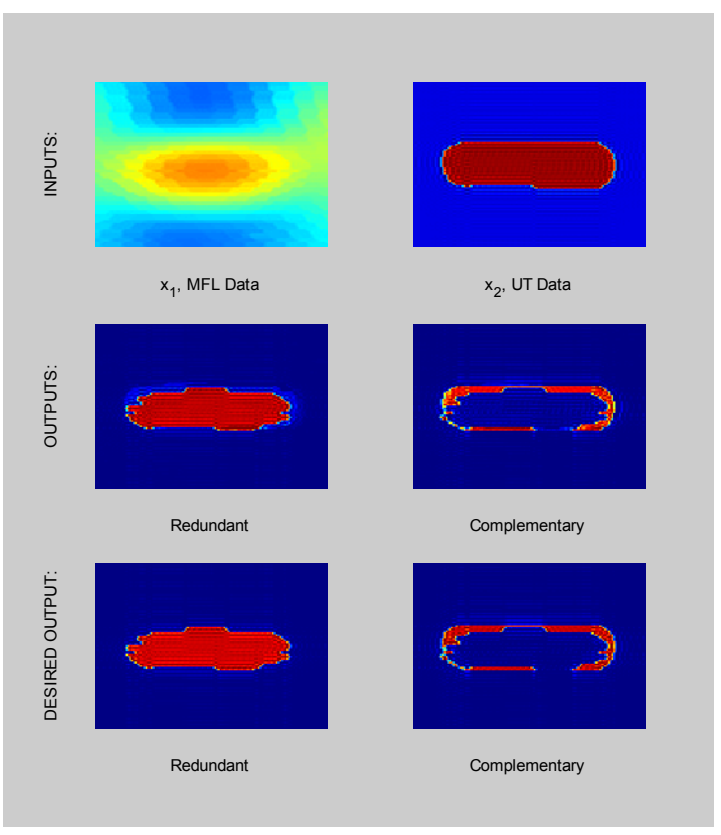

(h) Specimen 11a 
Trial 3: UT \& MFL Results (cont.)

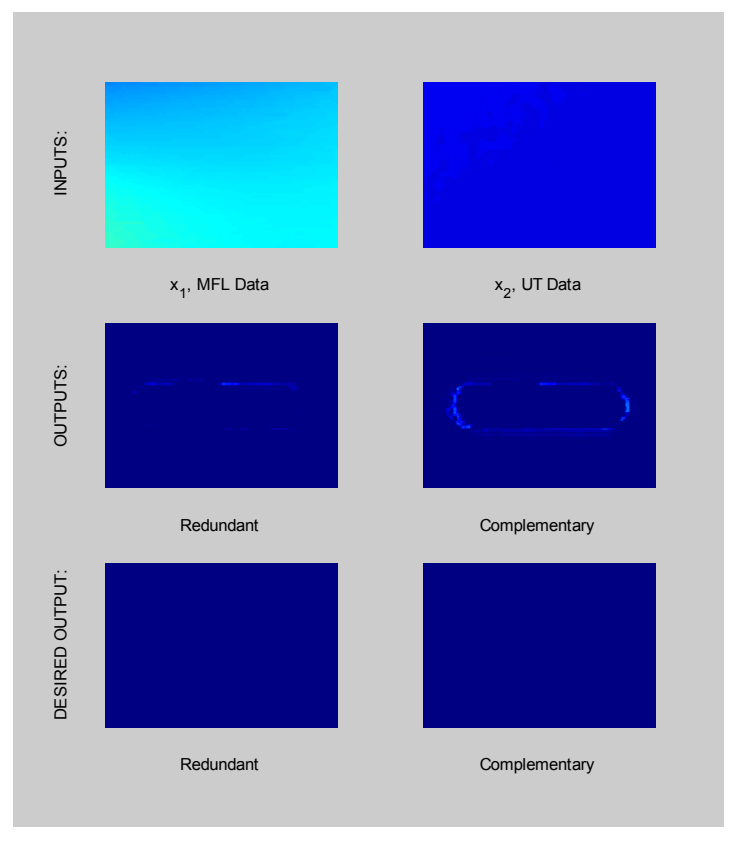

(i) Specimen 20a

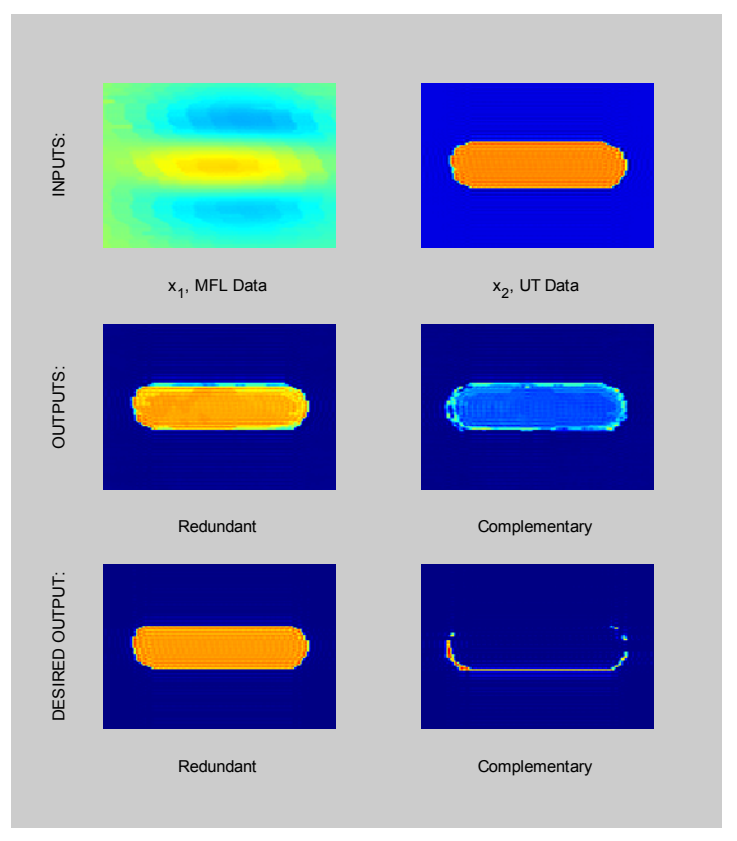

(k) Specimen 22a

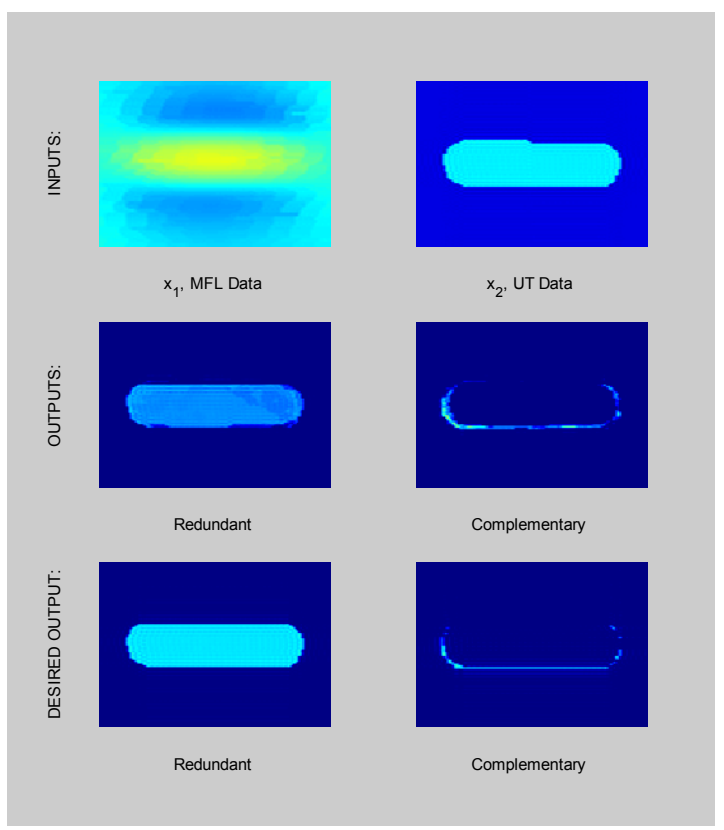

(j) Specimen 23a

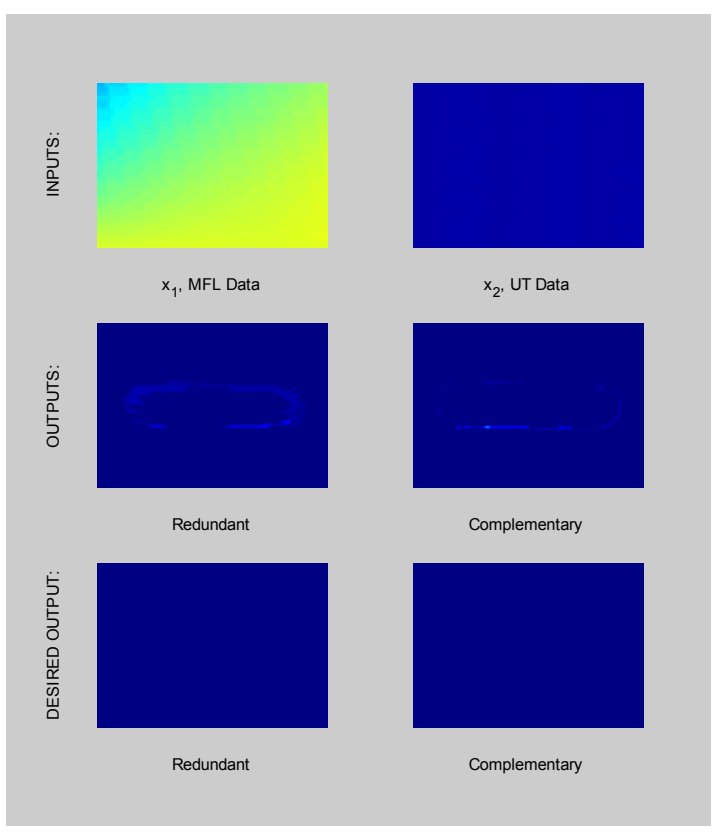

(l) Specimen 00b 
Trial 3: UT \& MFL Results (cont.)

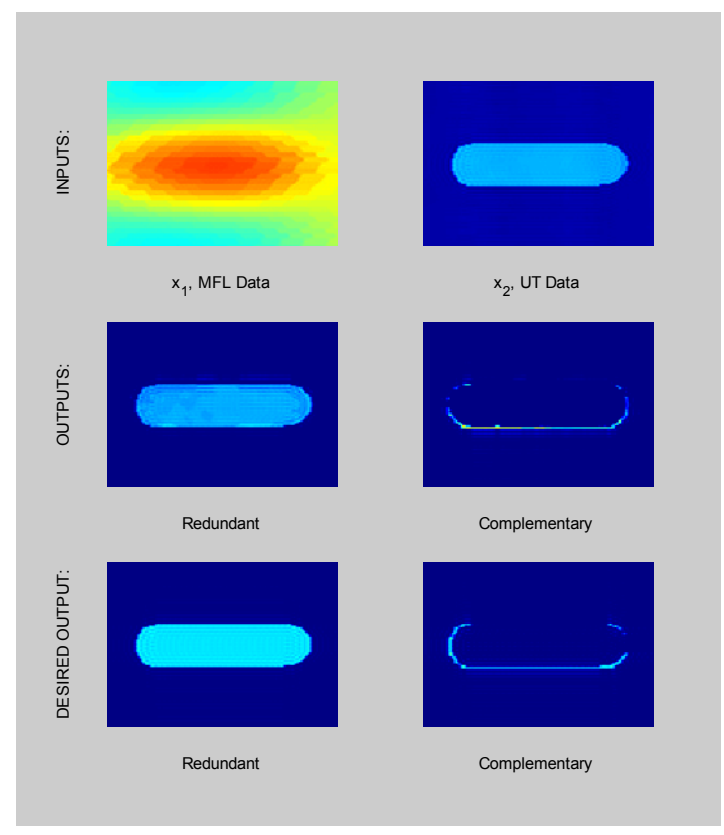

(m) Specimen 03b

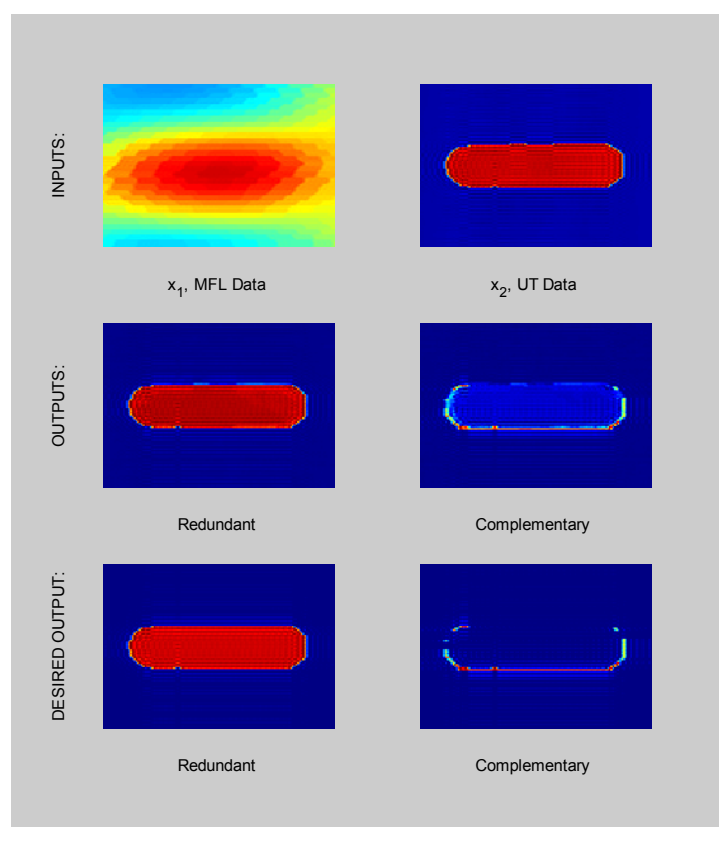

(o) Specimen 01b

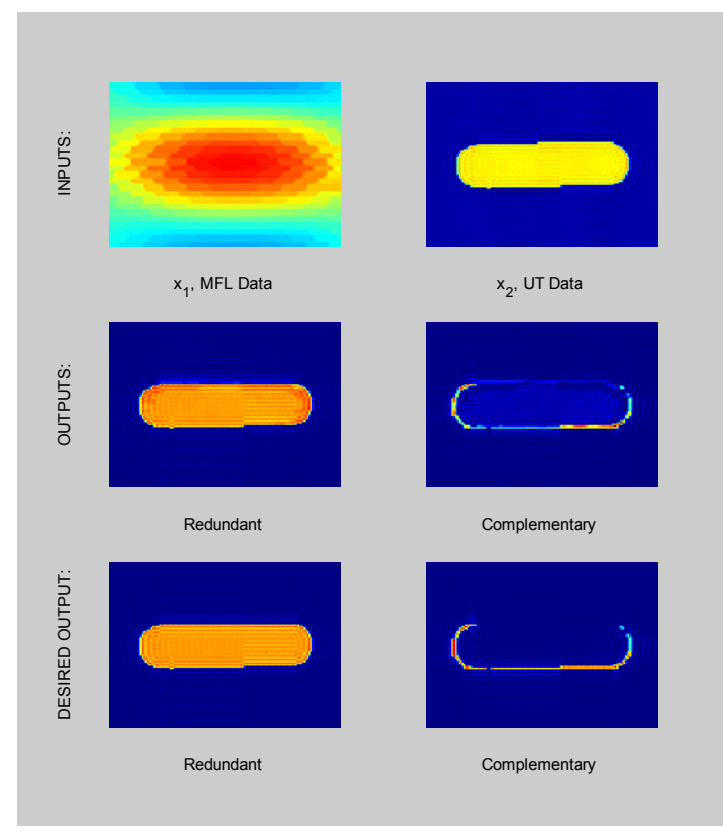

(n) Specimen 02b

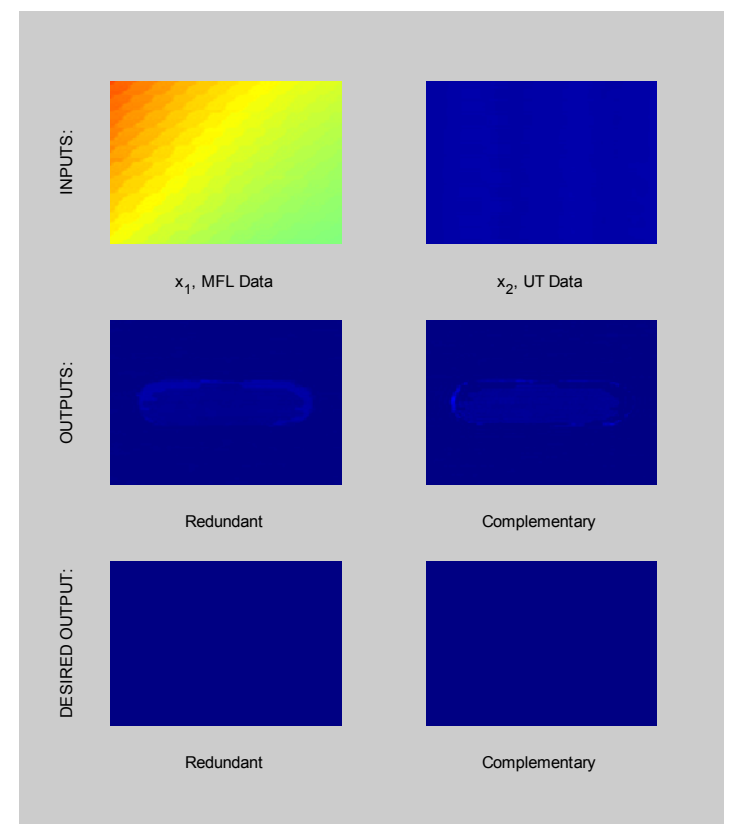

(p) Specimen 20b 
Trial 3: UT \& MFL Results (cont.)

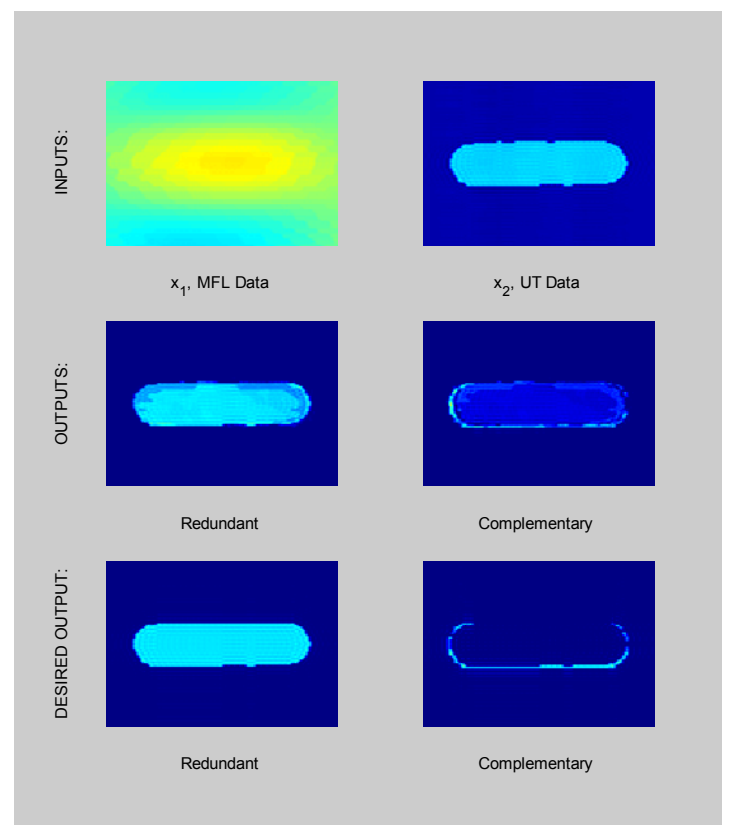

(q) Specimen 23b

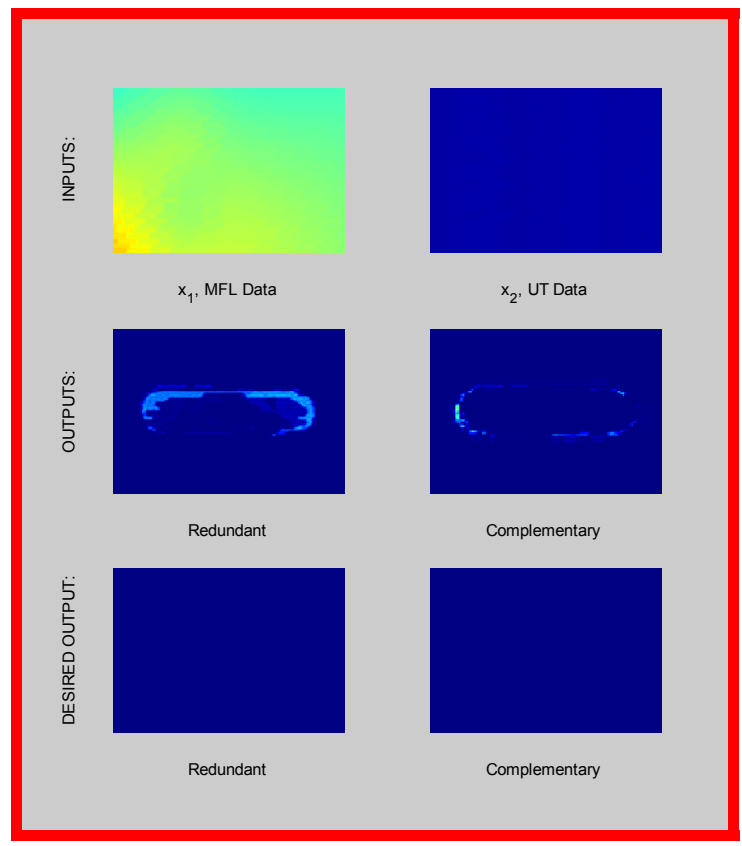

(s) Specimen 10b

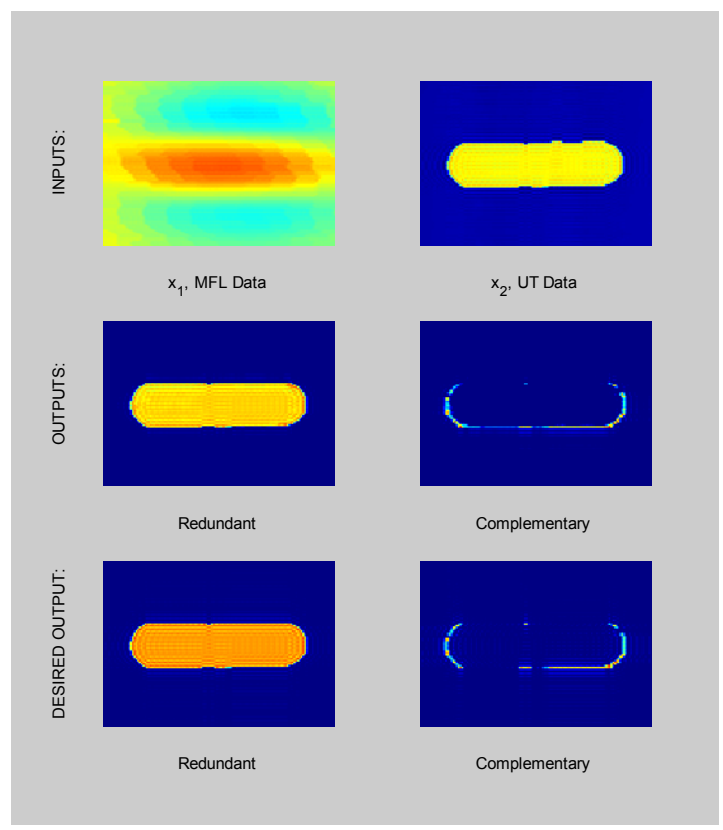

(r) Specimen 22b

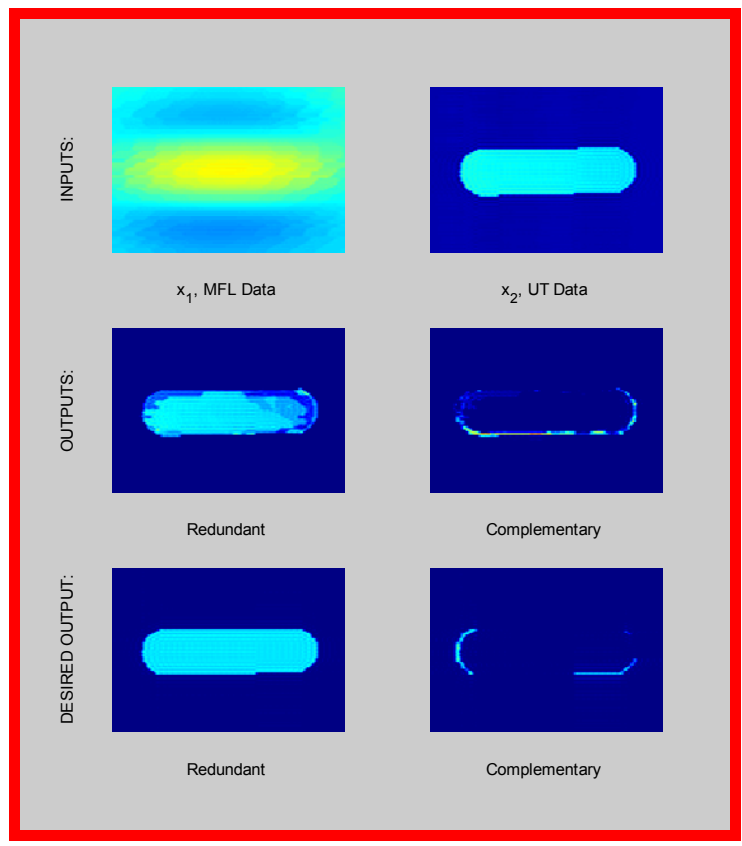

(t) Specimen 13b 
Trial 3: UT \& MFL Results (cont.)

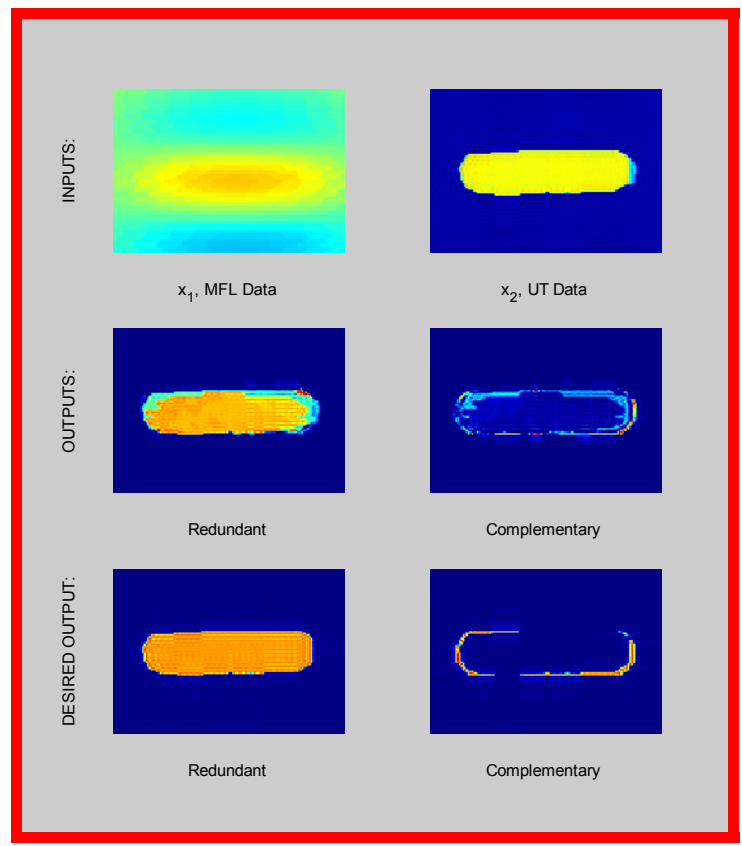

(u) Specimen 12b

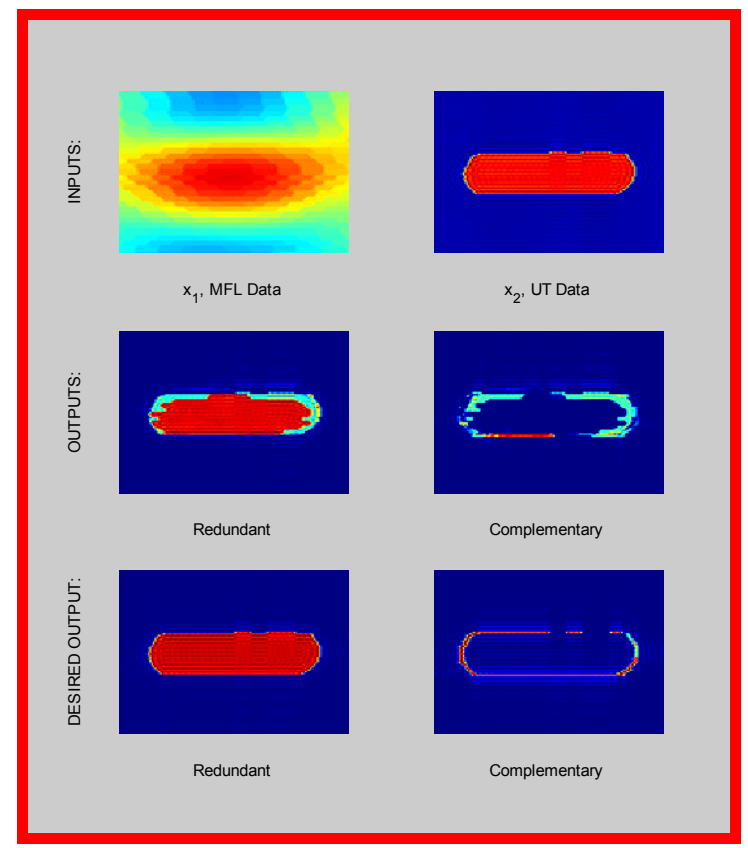

(v) Specimen 11b

Figure 4: UT \& MFL combination Trial 3.

Training Data: (a) - (r); Test Data: (s), (t), (u), (v) 
Trial 1: Thermal \& UT Results

\section{Thermal \& UT Data Fusion Results}

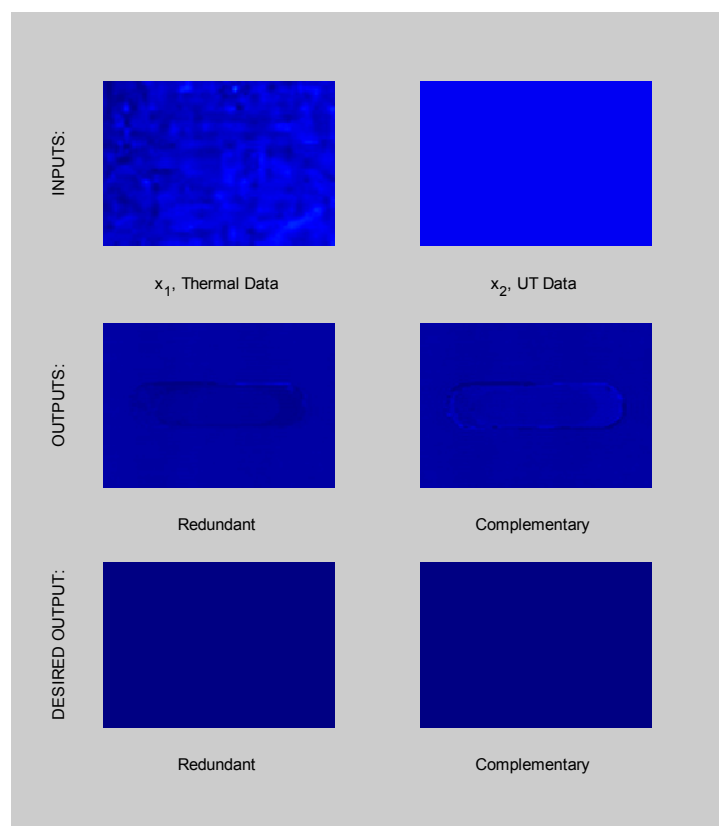

(a) Specimen 00a

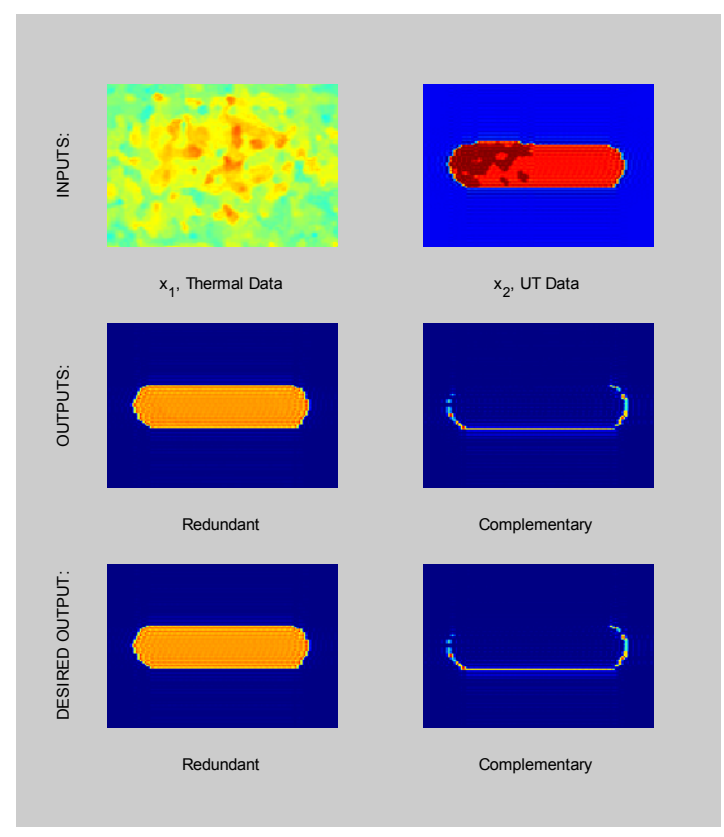

(c) Specimen 02a

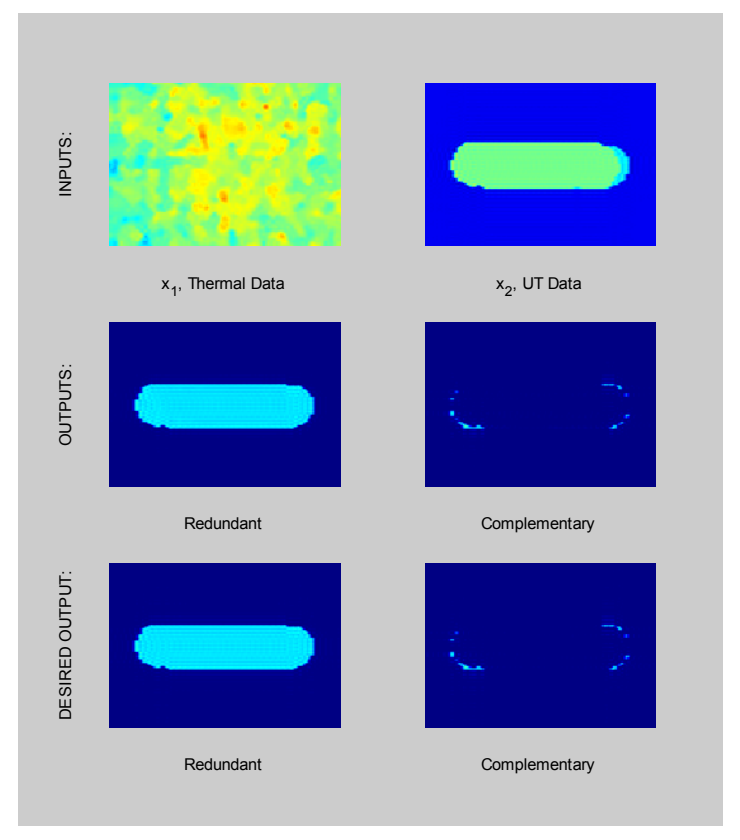

(b) Specimen 03a

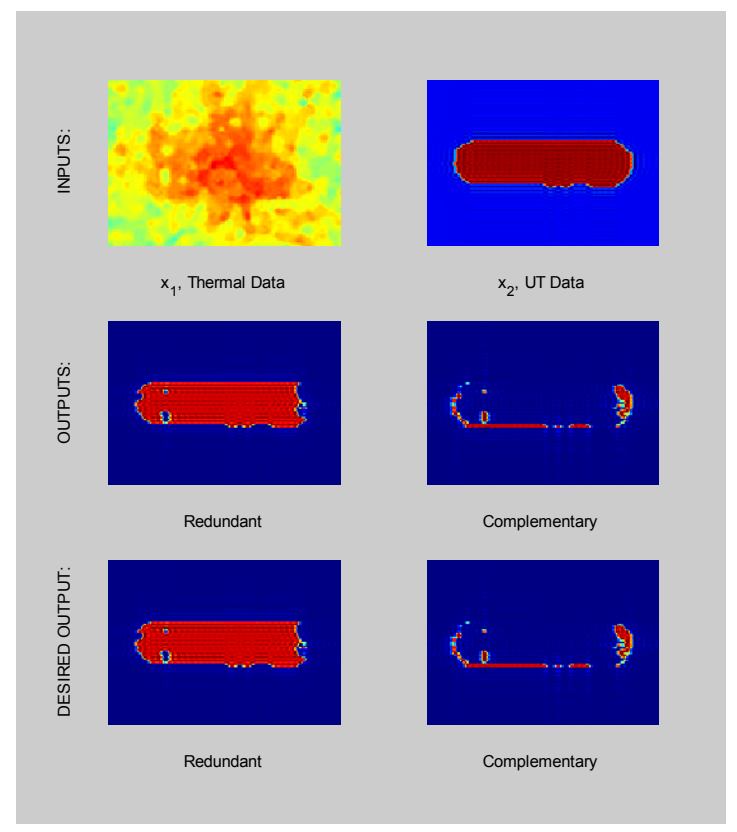

(d) Specimen 01a 
Trial 1: Thermal \& UT Results (cont.)

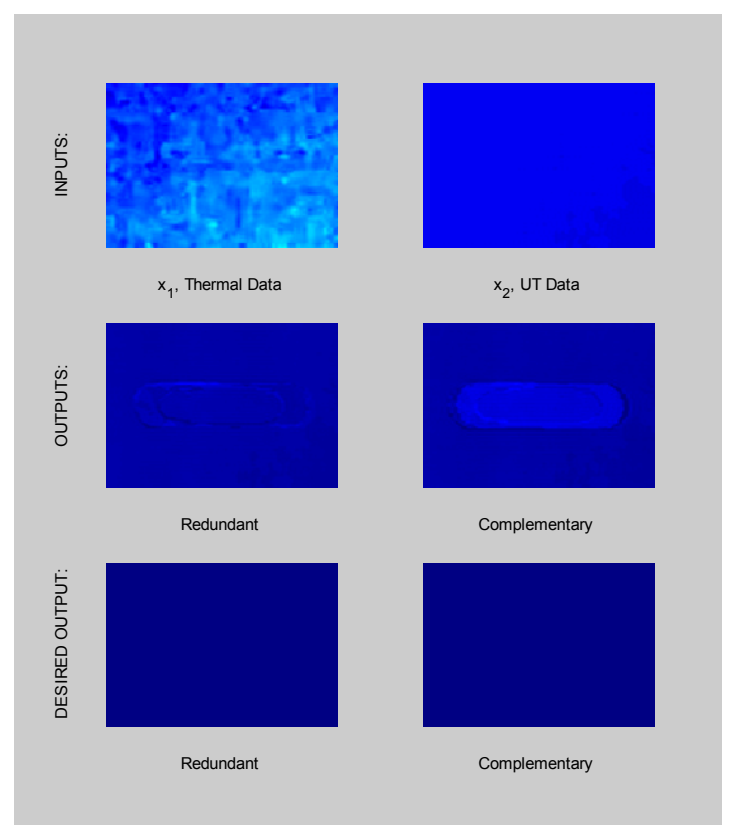

(e) Specimen 10a

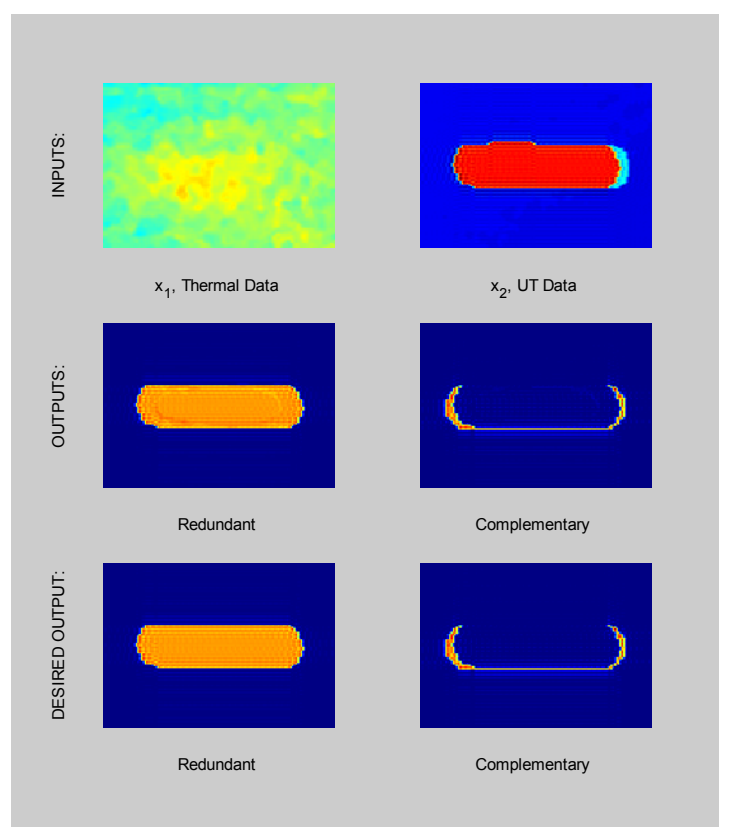

(g) Specimen 12a

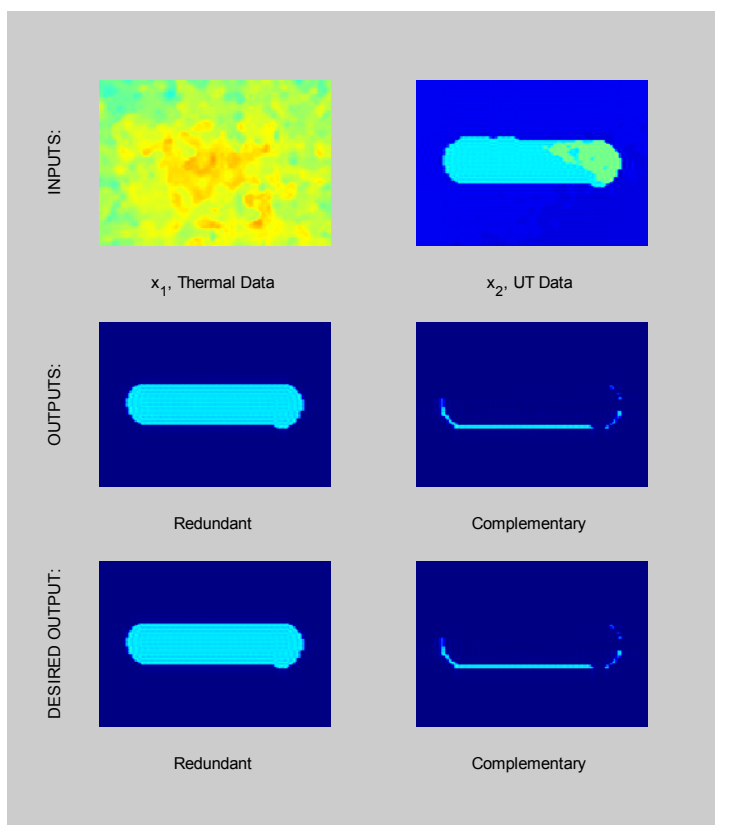

(f) Specimen 13a

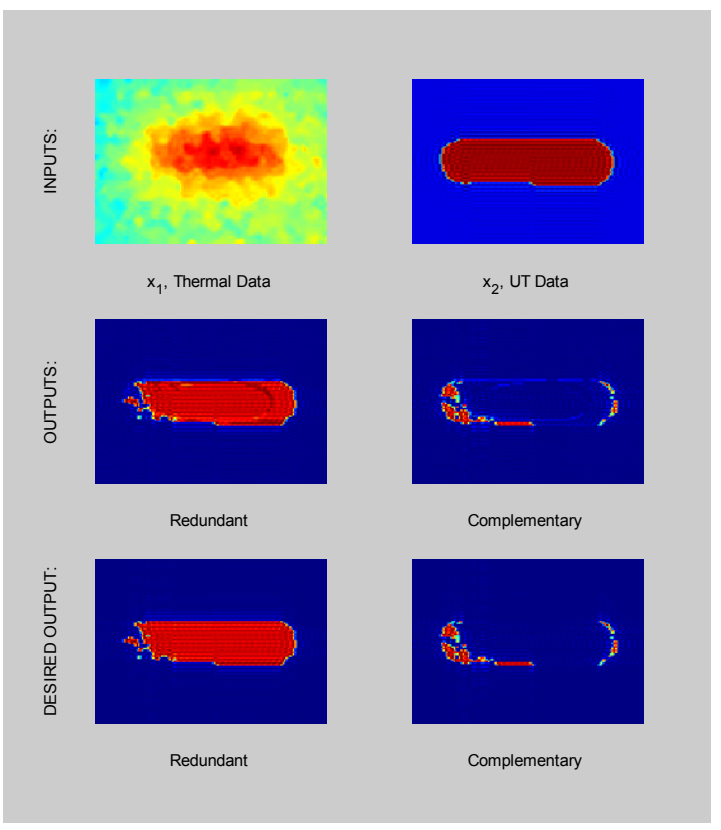

(h) Specimen 11a 
Trial 1: Thermal \& UT Results (cont.)

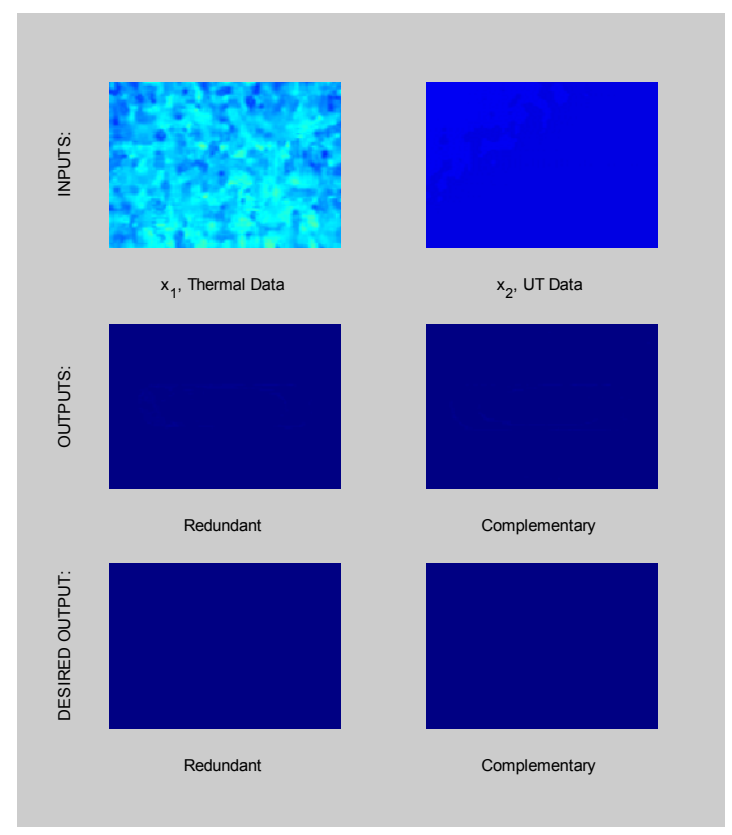

(i) Specimen 20a

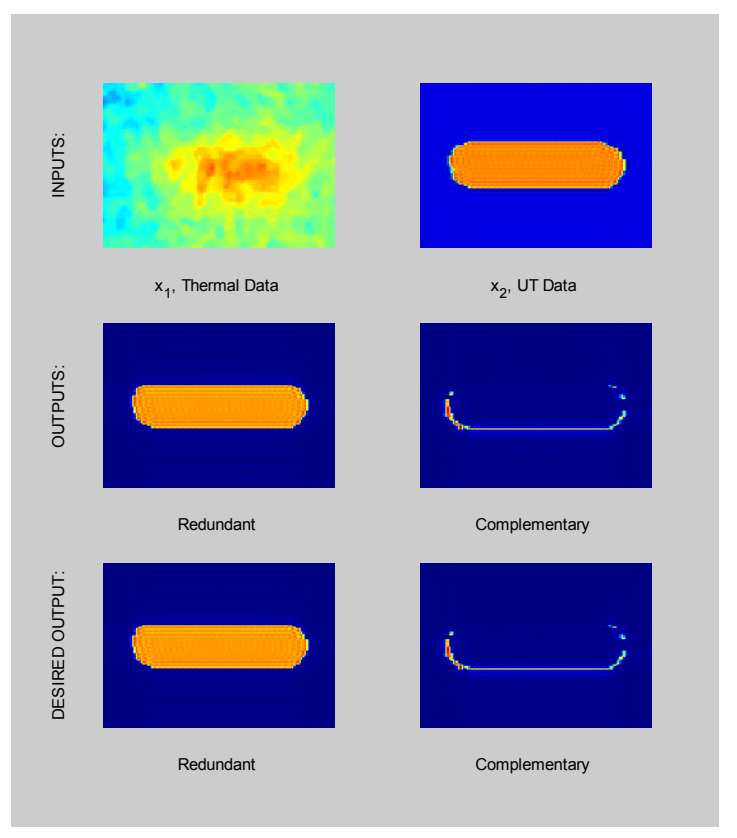

(k) Specimen 22a

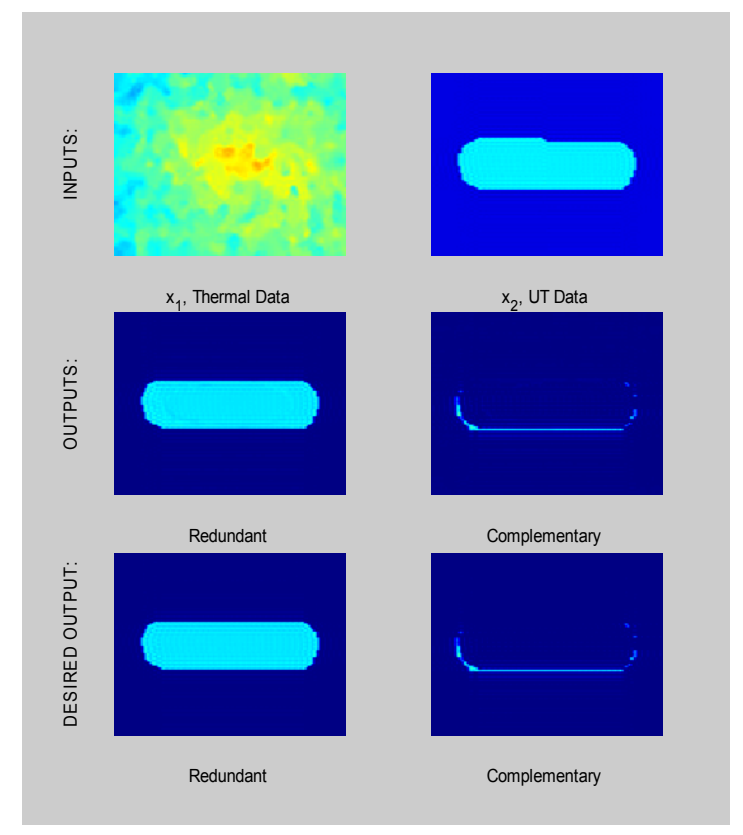

(j) Specimen 23a

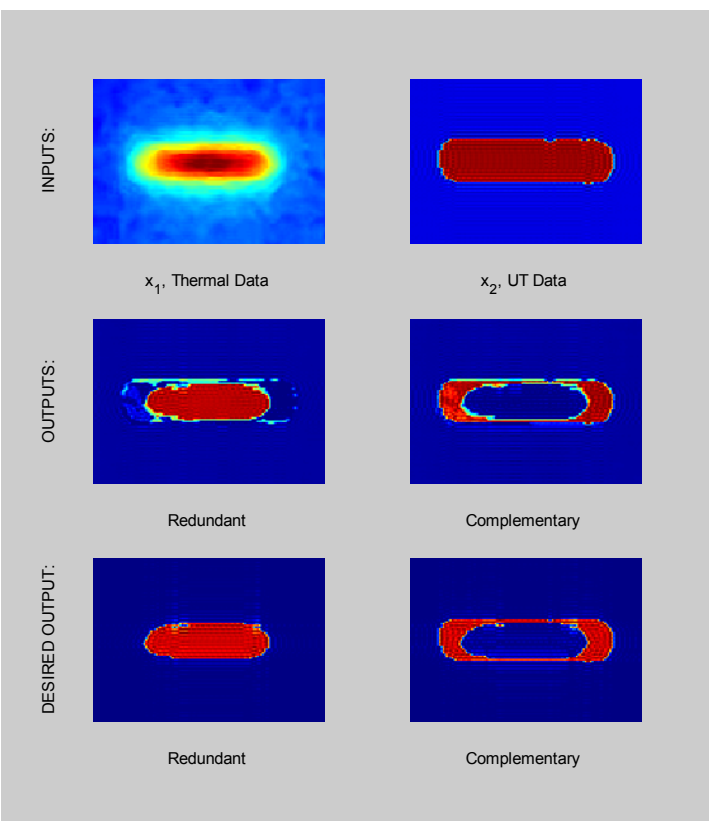

(l) Specimen 21a 
Trial 1: Thermal \& UT Results (cont.)

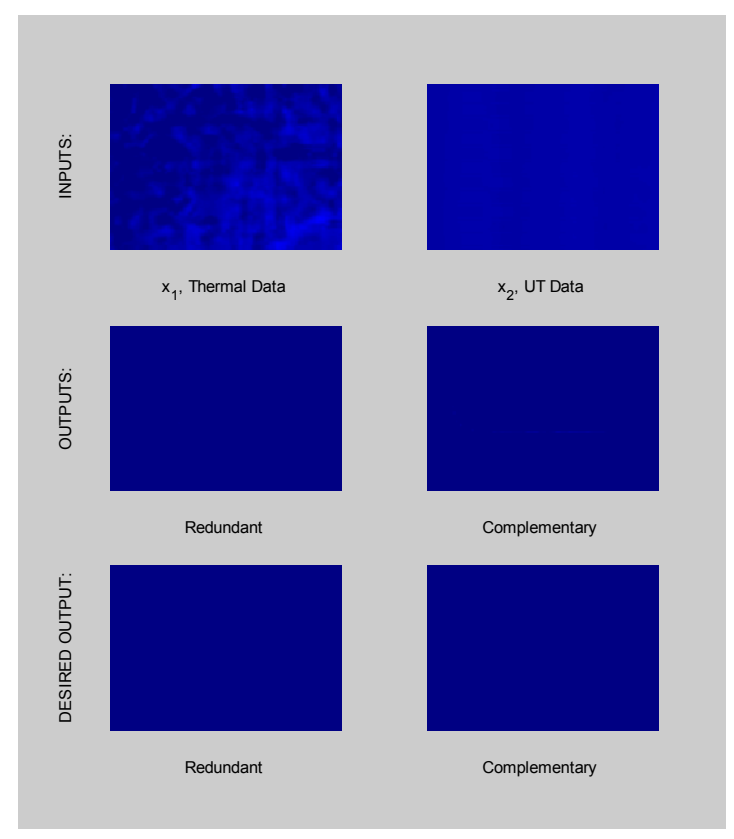

(m) Specimen 00b

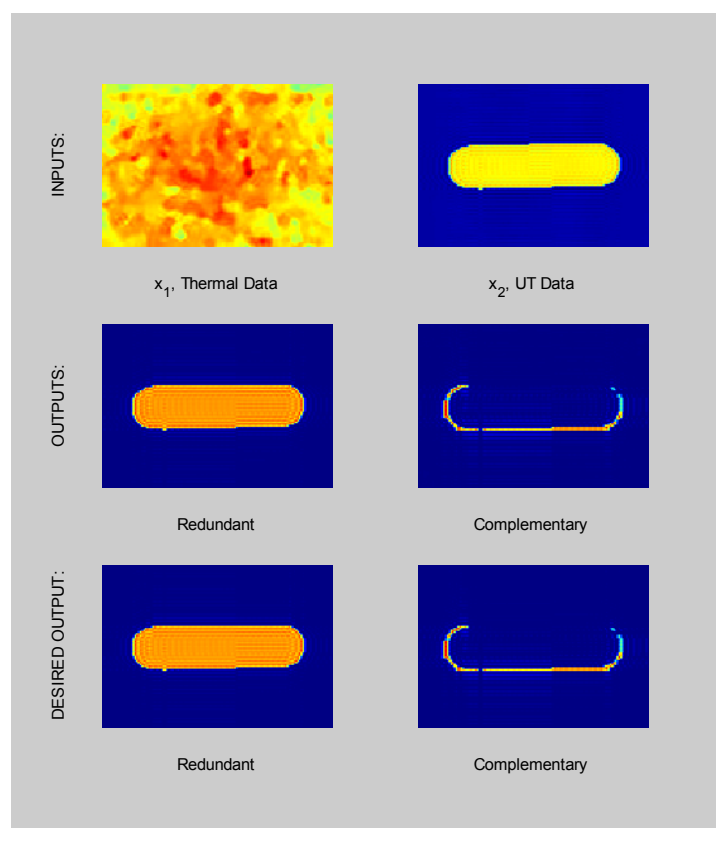

(o) Specimen 02b

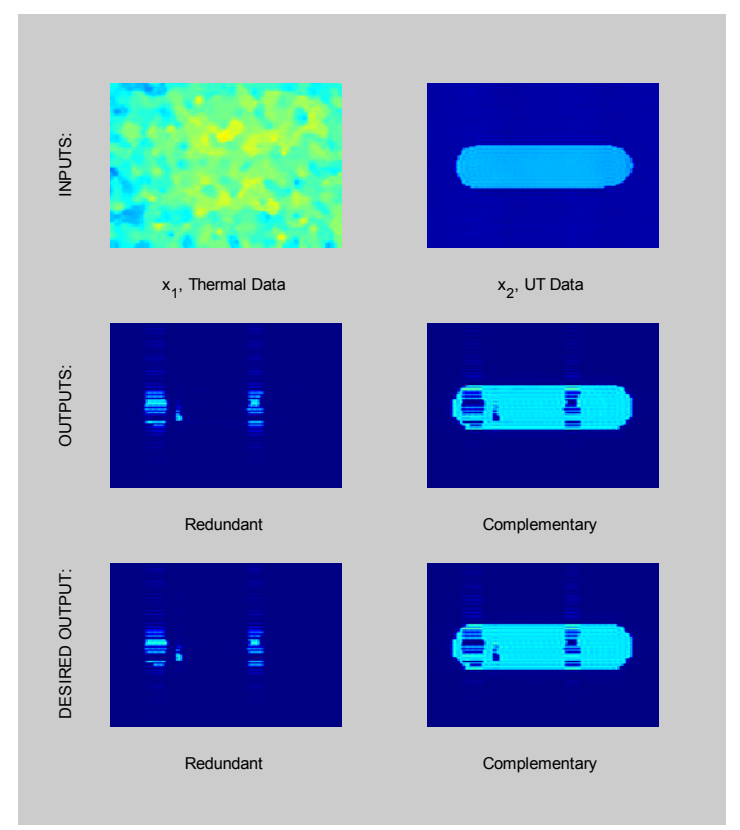

(n) Specimen 03b

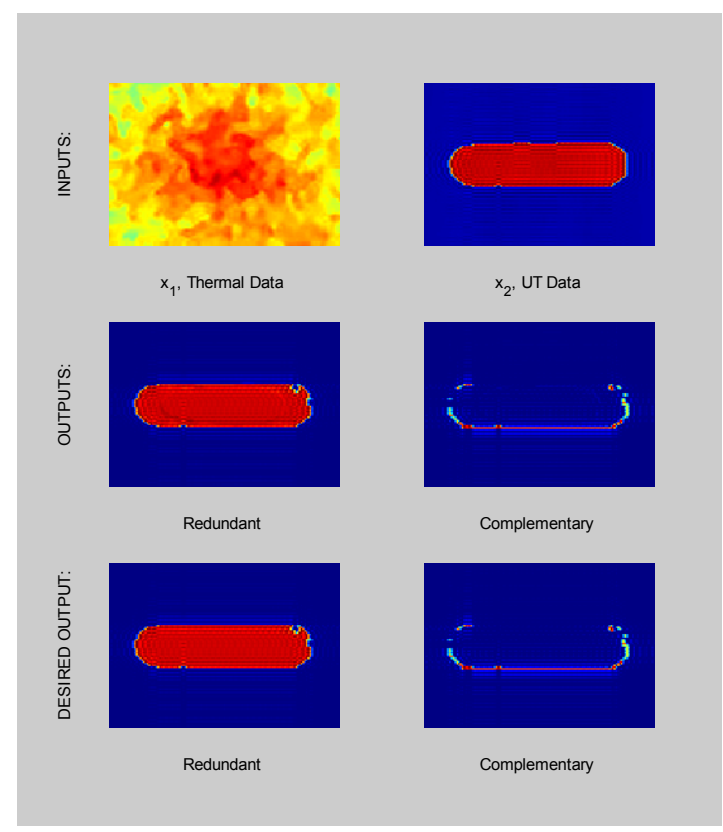

(p) Specimen 01b 
Trial 1: Thermal \& UT Results (cont.)

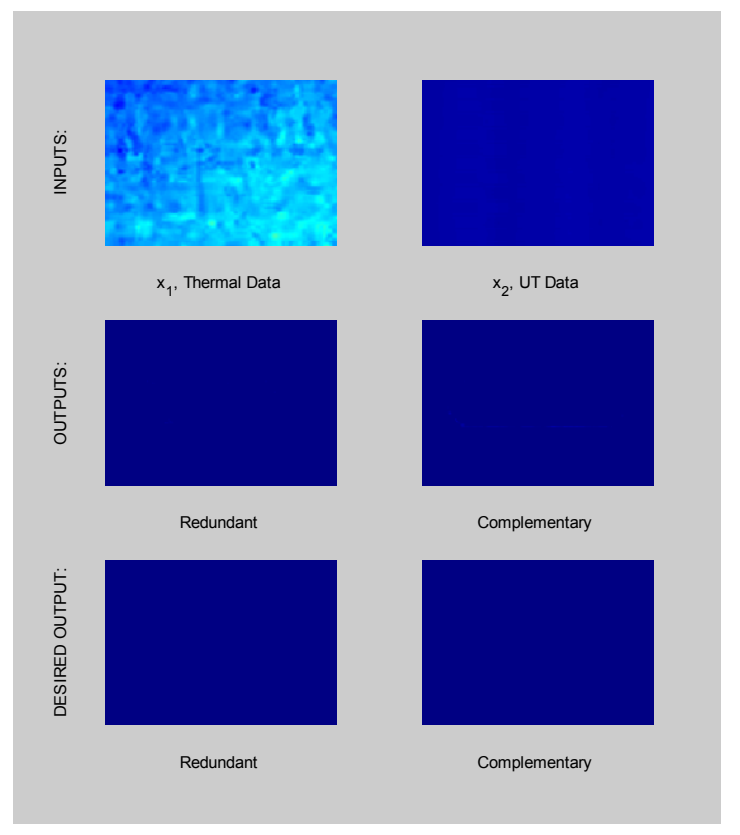

(q) Specimen 10b

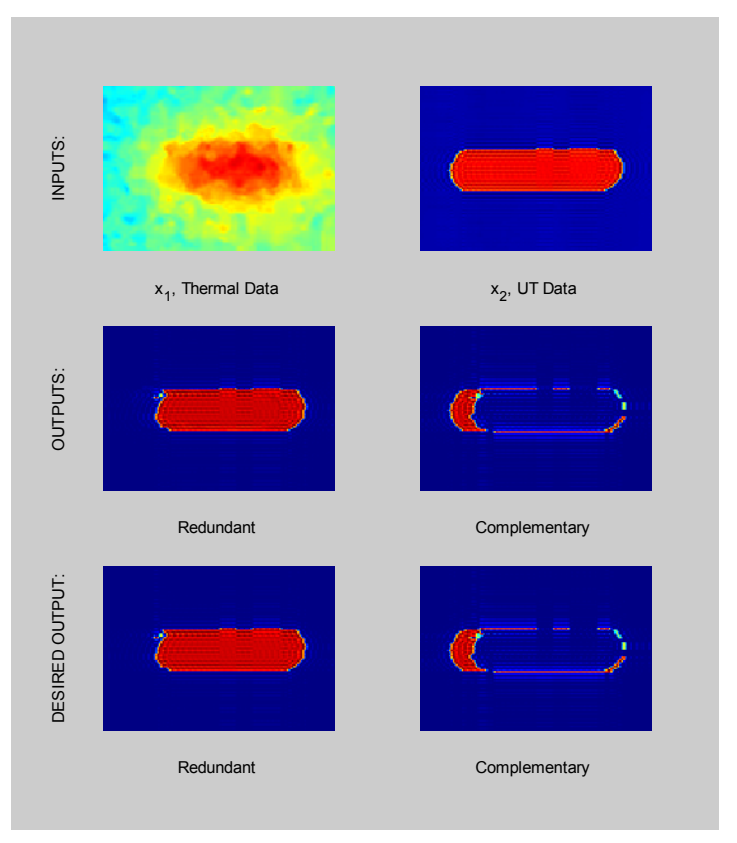

(s) Specimen 11b

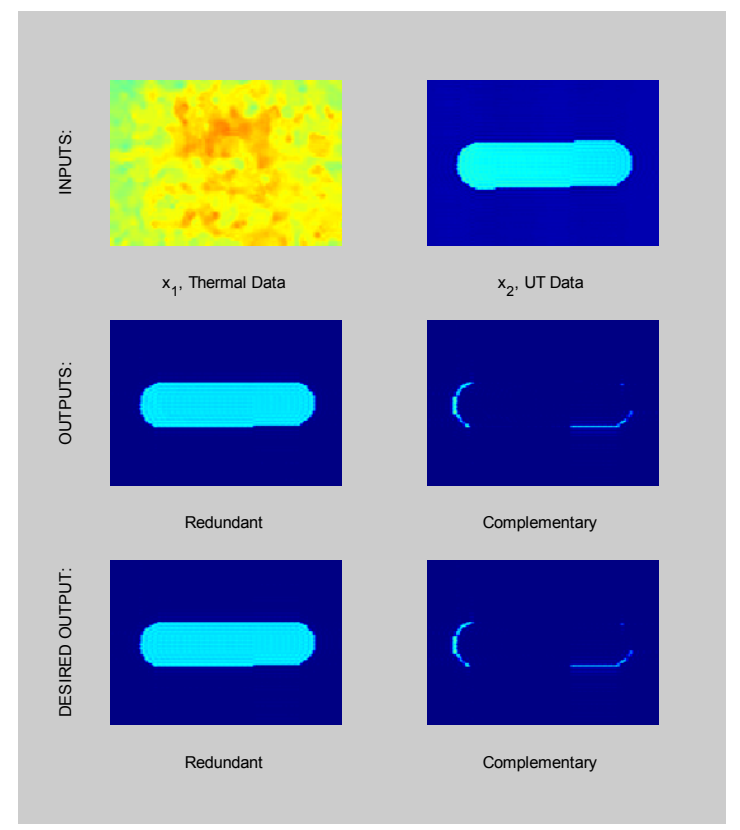

(r) Specimen 13b

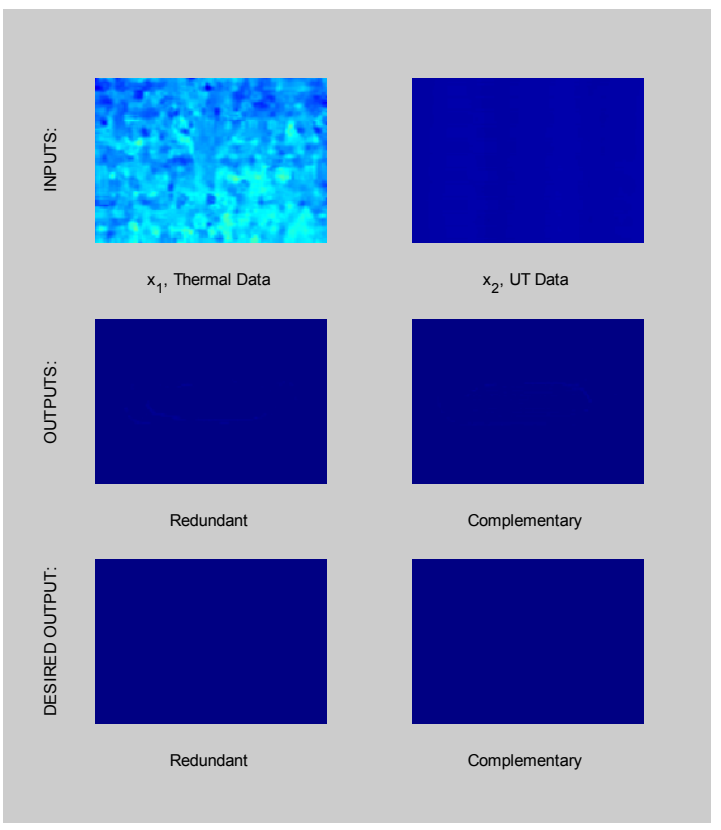

(t) Specimen 20b 
Trial 1: Thermal \& UT Results (cont.)

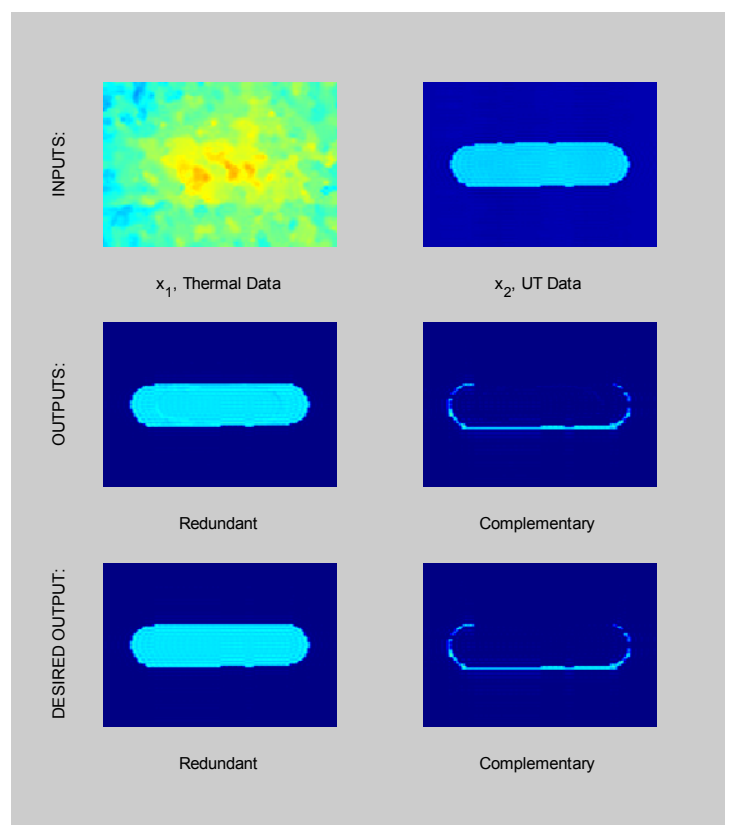

(u) Specimen 23b

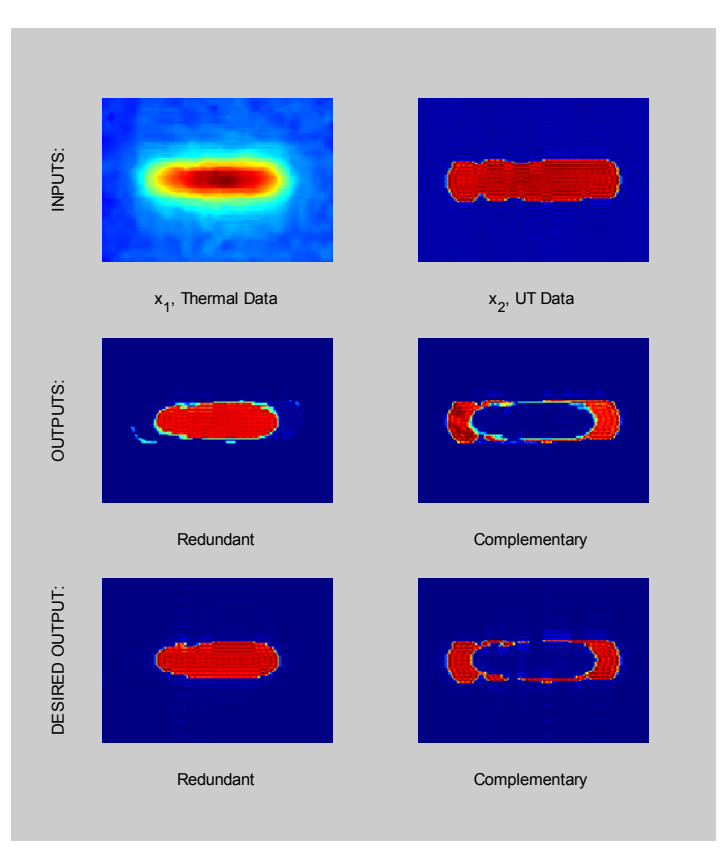

(w) Specimen 21b

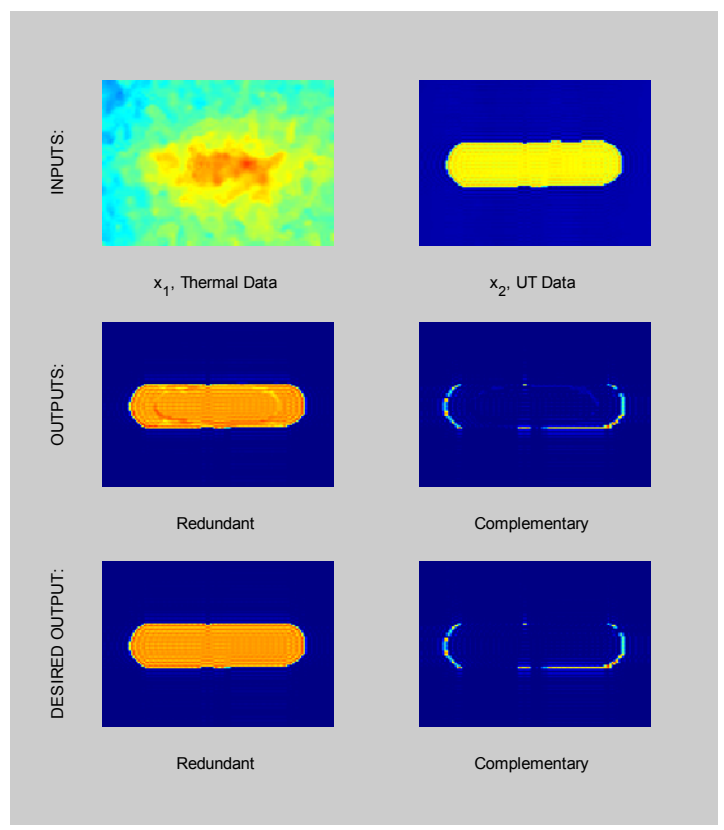

(v) Specimen 22b

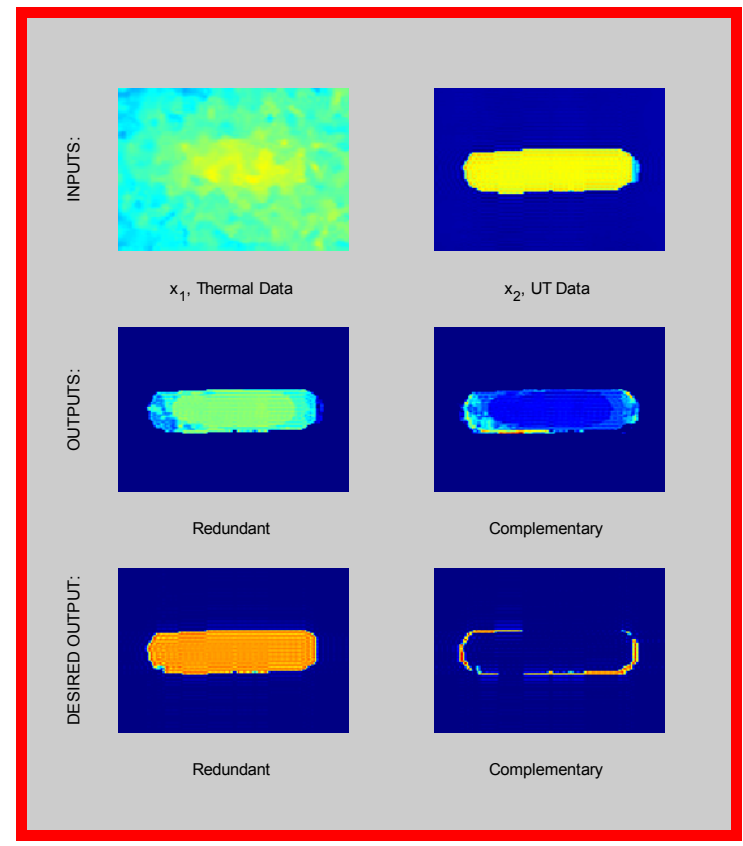

(x) Specimen 12b

Figure 5: Thermal \& UT combination Trial 1.

Training Data: (a) - (w); Test Data: (x) 
Trial 2: Thermal \& UT Results

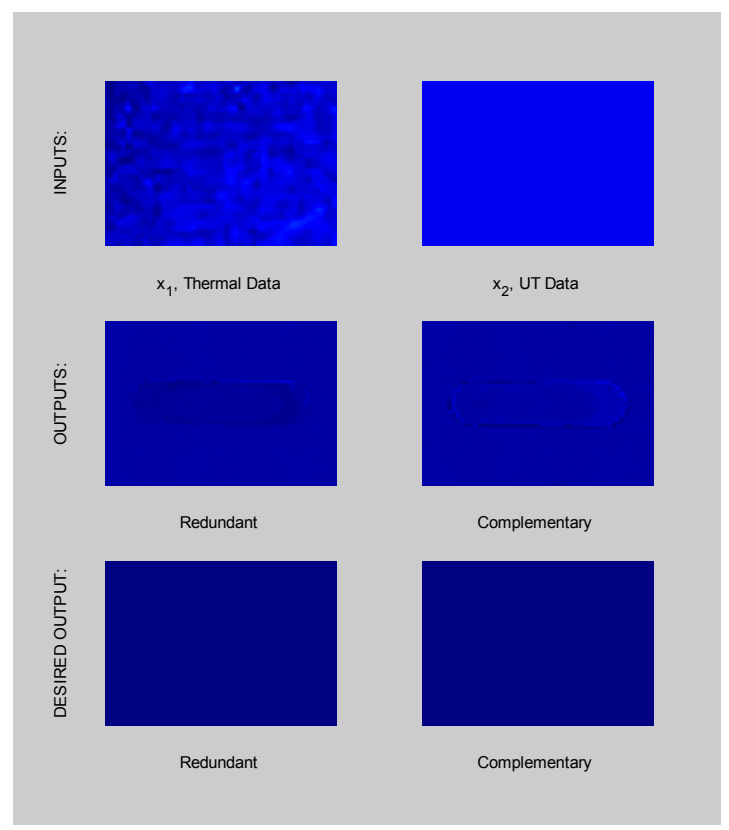

(a) Specimen 00a

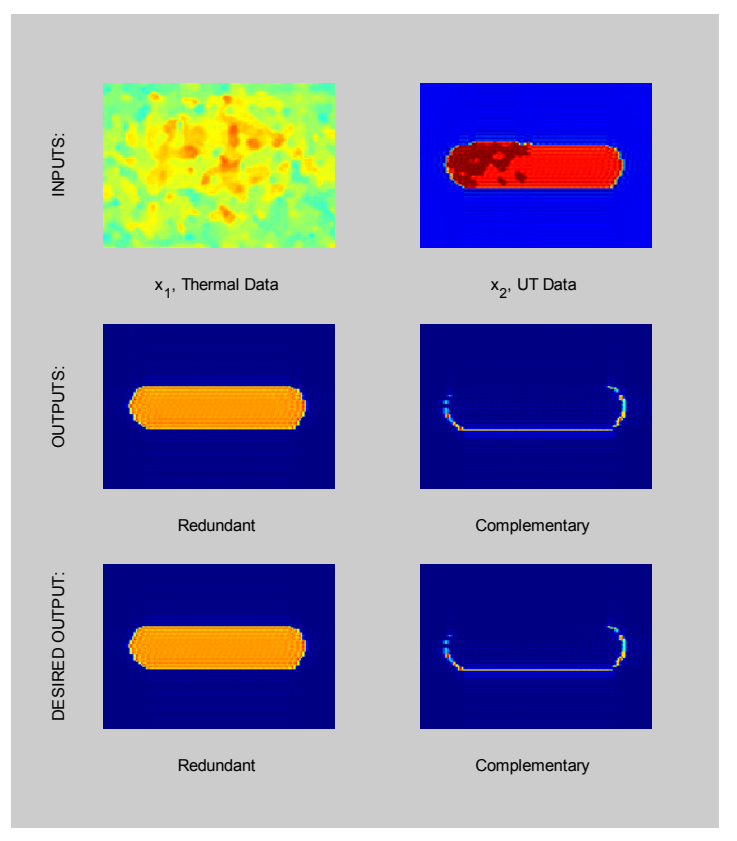

(c) Specimen 02a

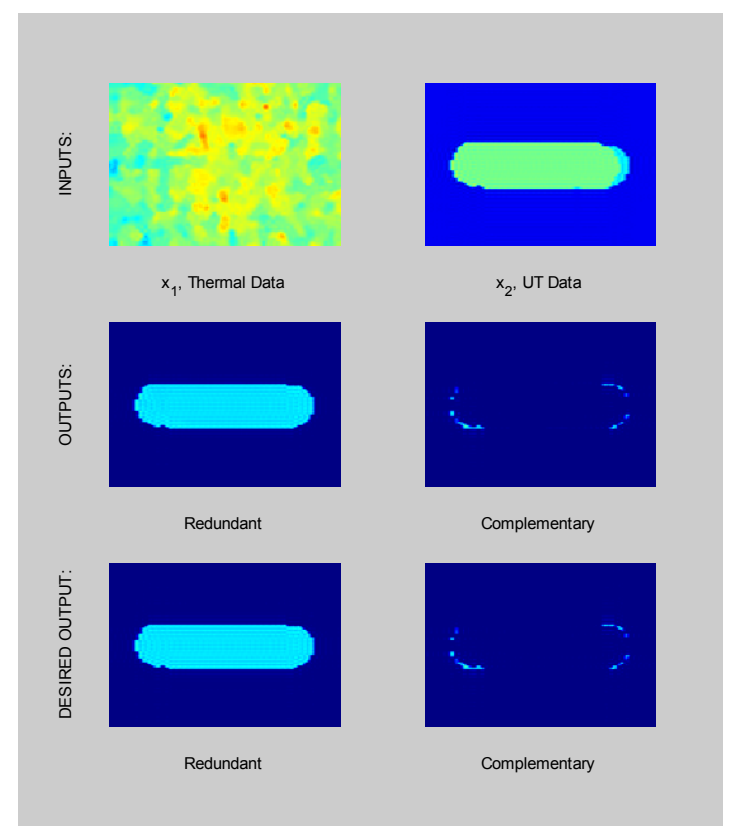

(b) Specimen 03a

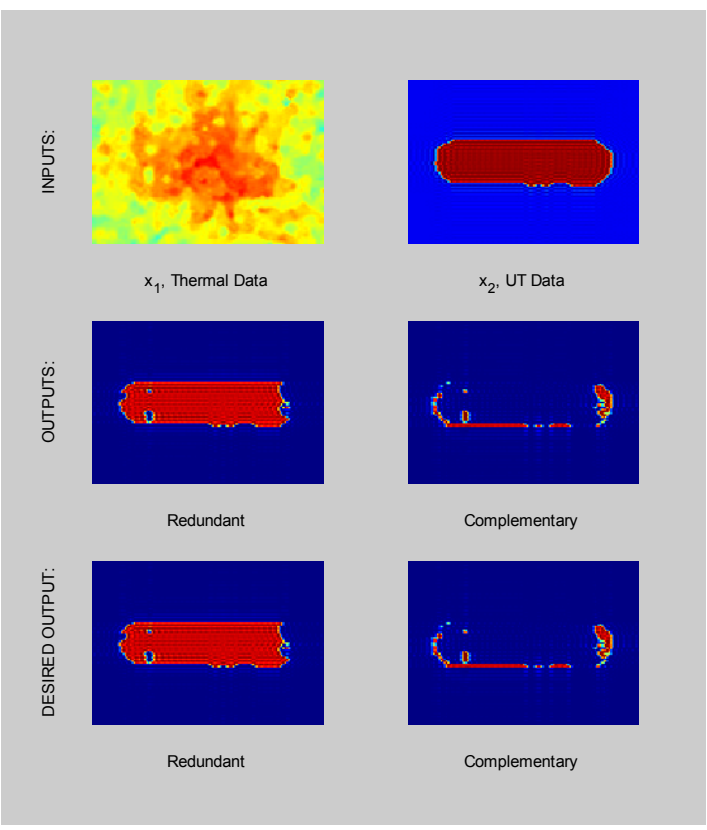

(d) Specimen 01a 
Trial 2: Thermal \& UT Results (cont.)

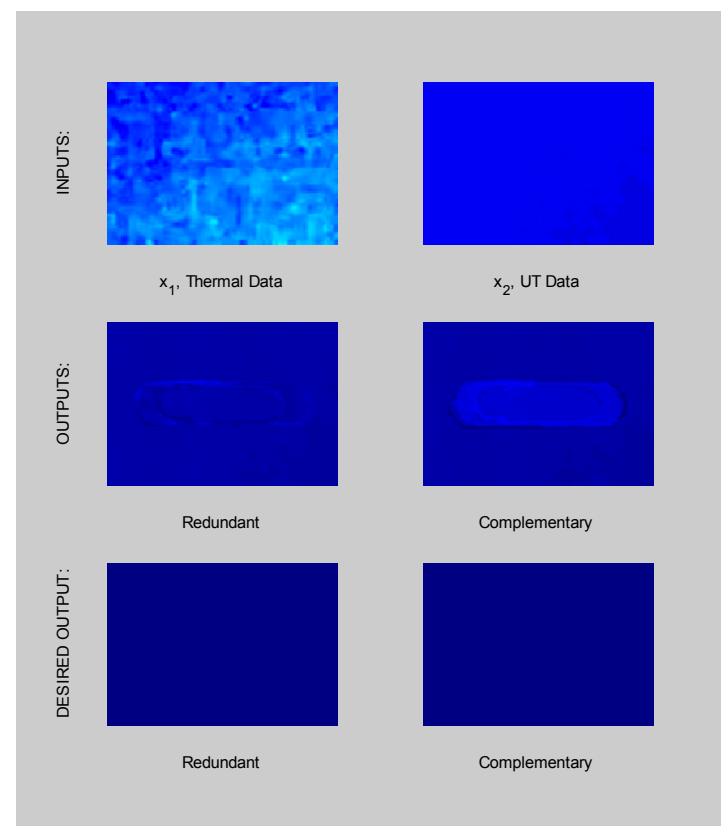

(e) Specimen 10a

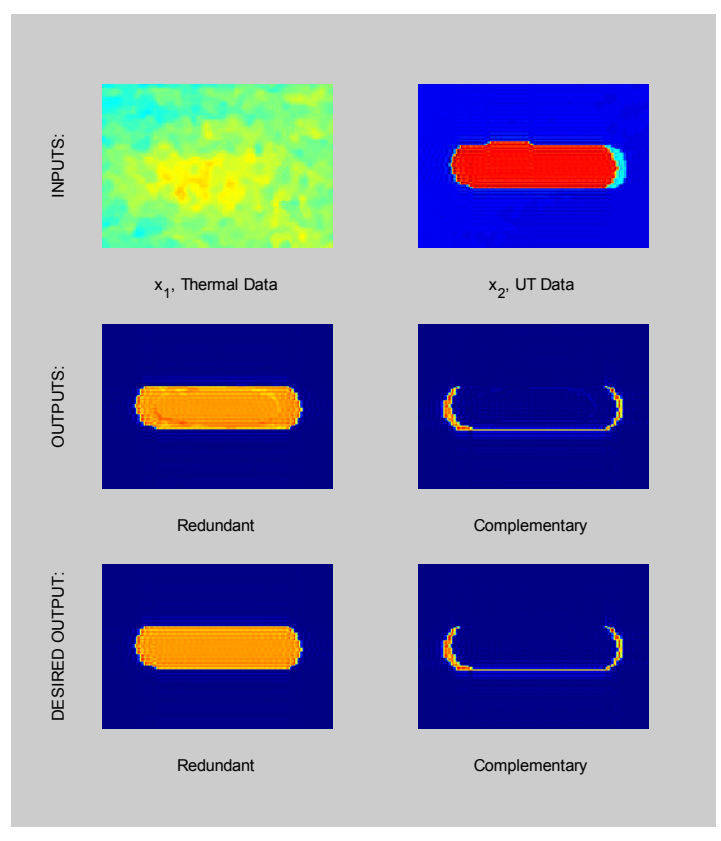

(g) Specimen 12a

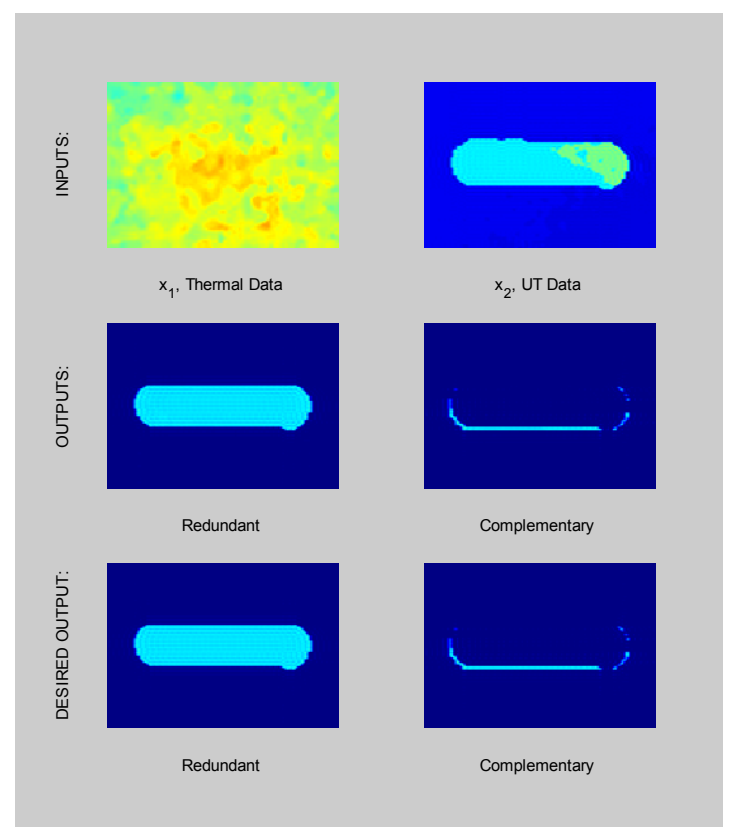

(f) Specimen 13a

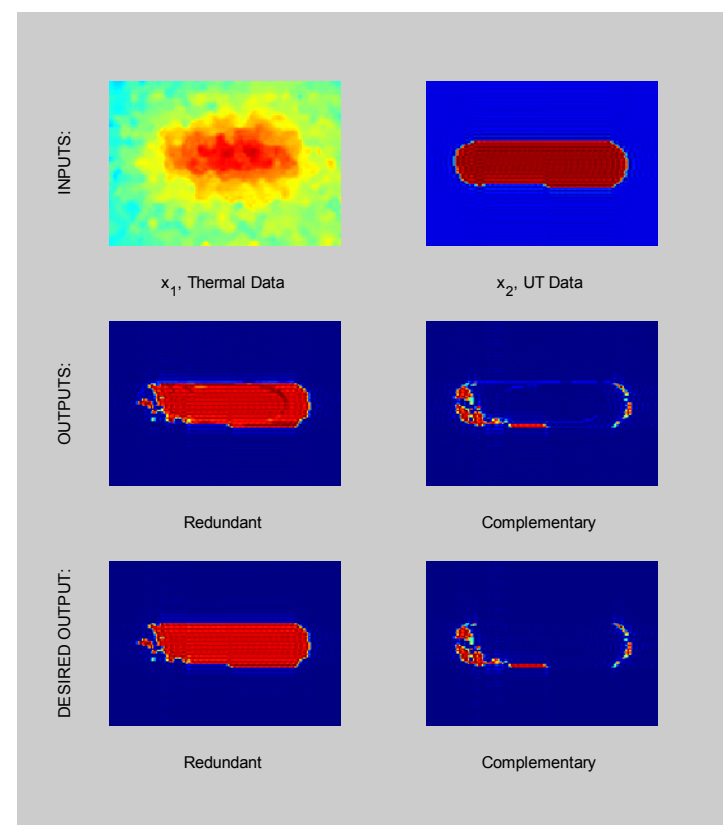

(h) Specimen 11a 
Trial 2: Thermal \& UT Results (cont.)

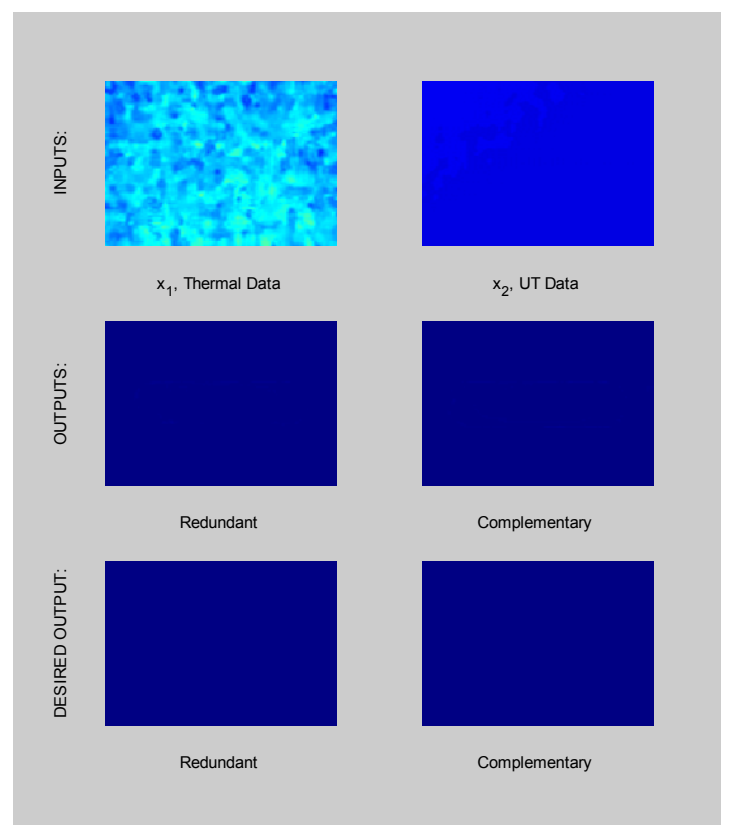

(i) Specimen 20a

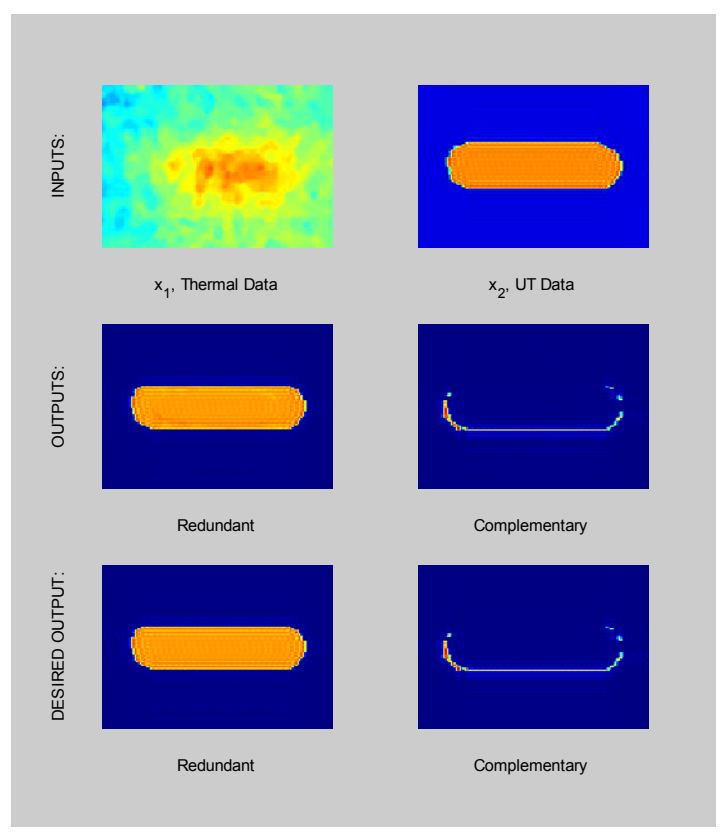

(k) Specimen 22a

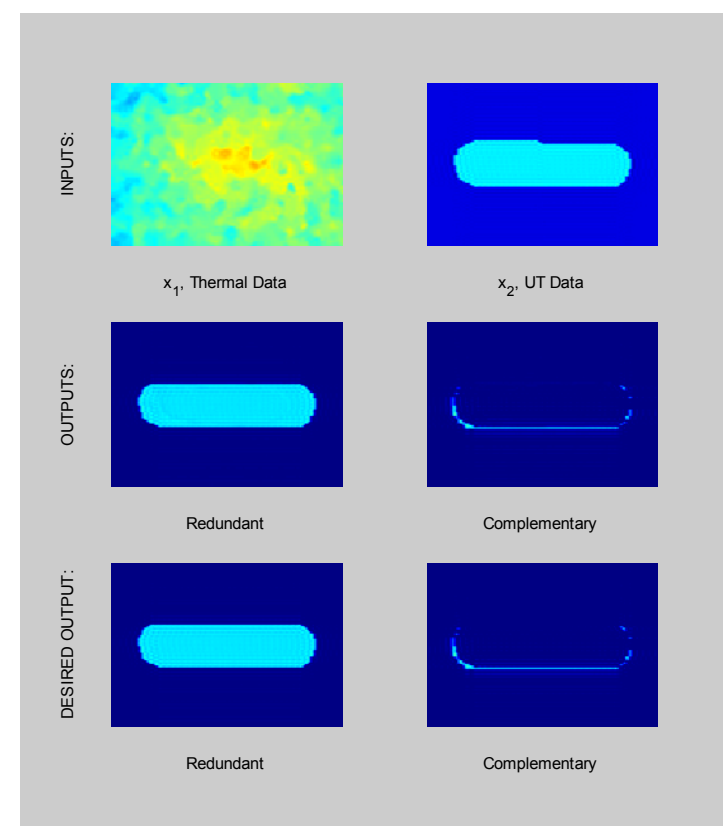

(j) Specimen 23a

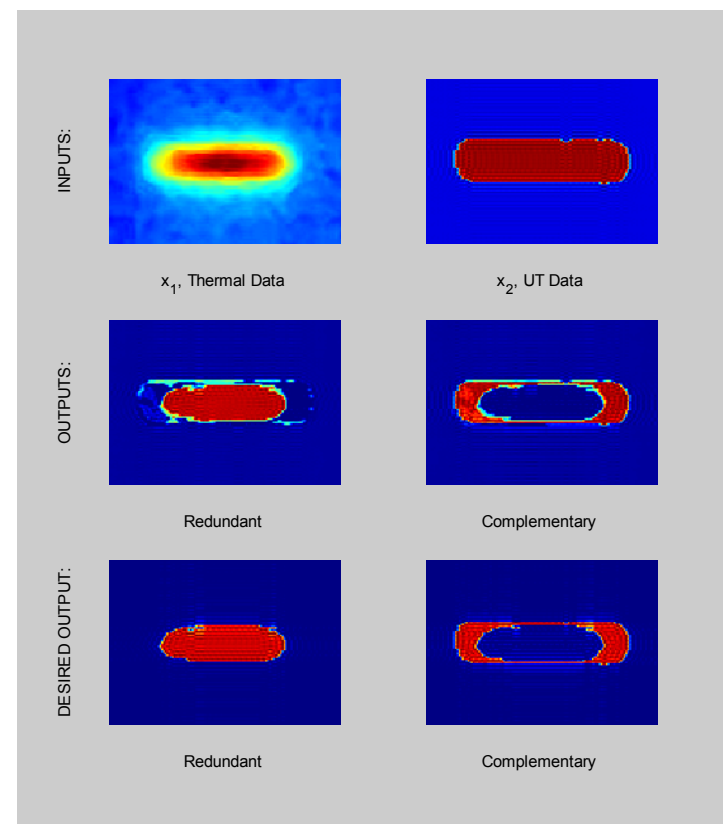

(l) Specimen 21a 
Trial 2: Thermal \& UT Results (cont.)

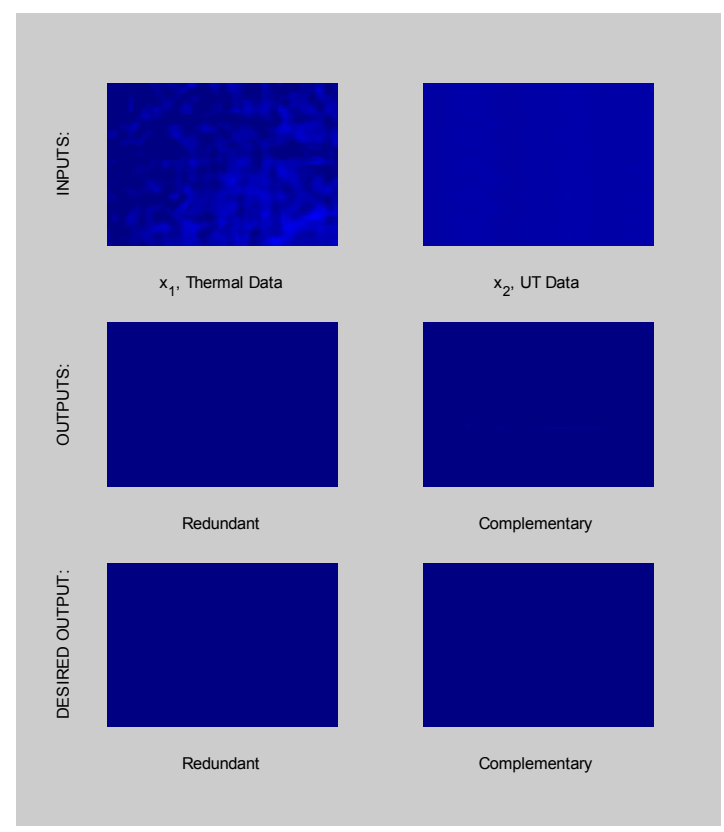

(m) Specimen 00b

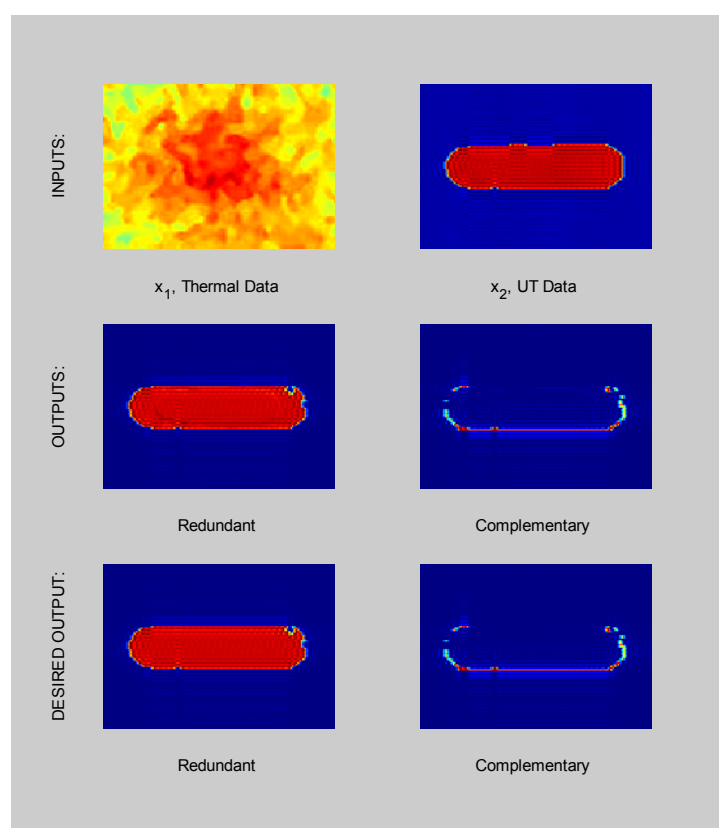

(o) Specimen 01b

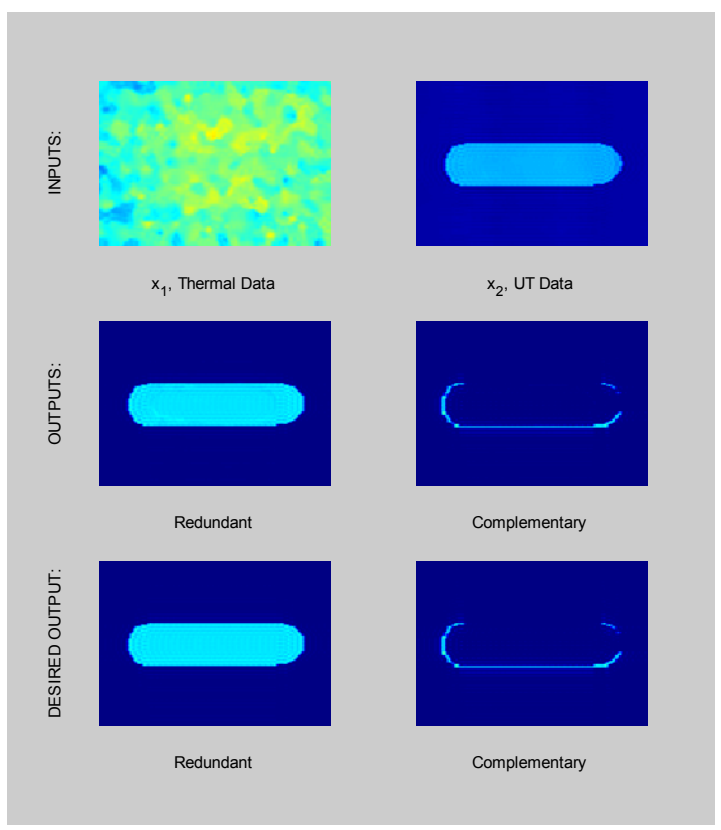

(n) Specimen 03b

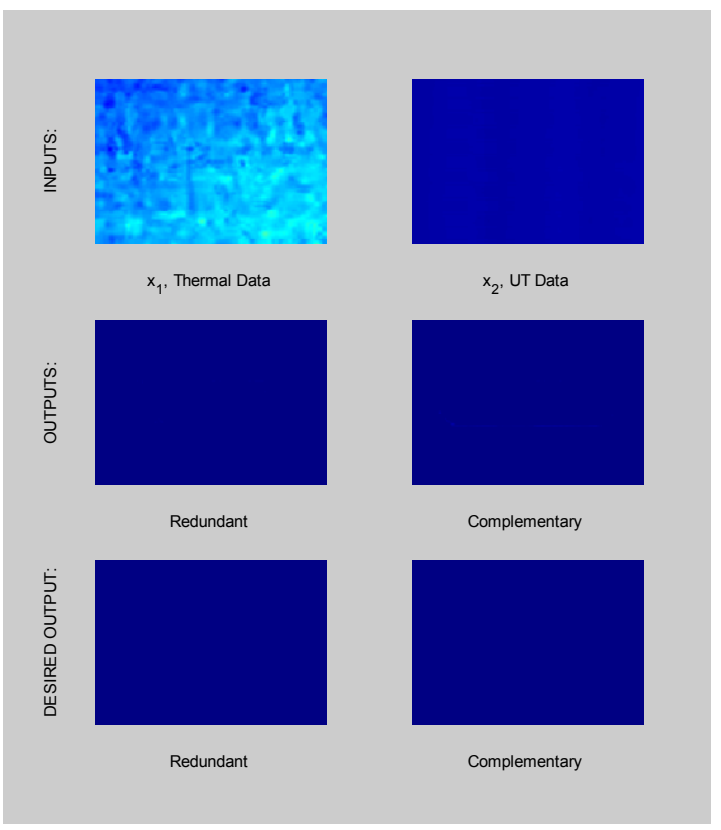

(p) Specimen 10b 
Trial 2: Thermal \& UT Results (cont.)

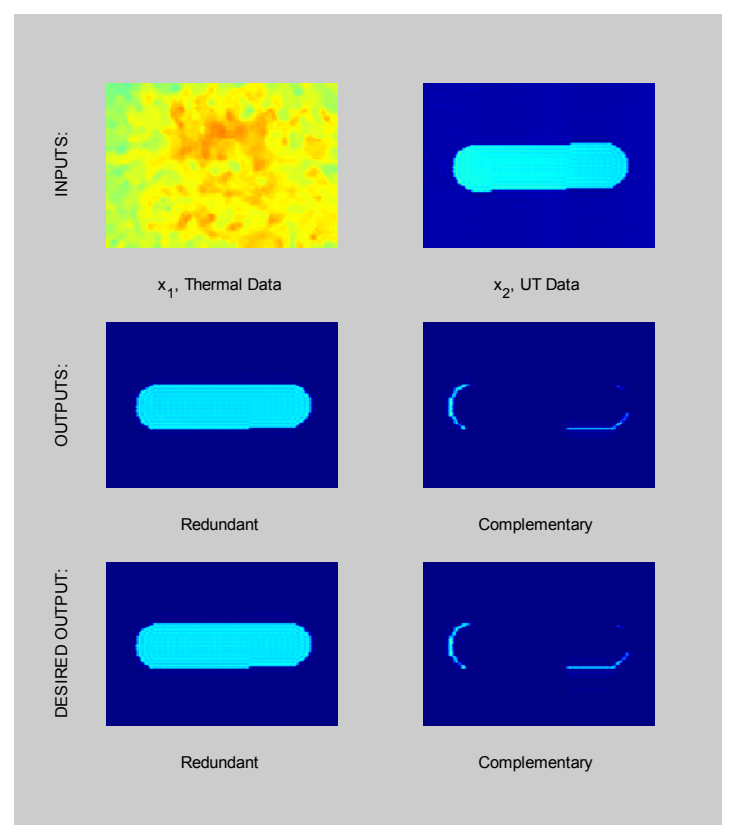

(q) Specimen 13b

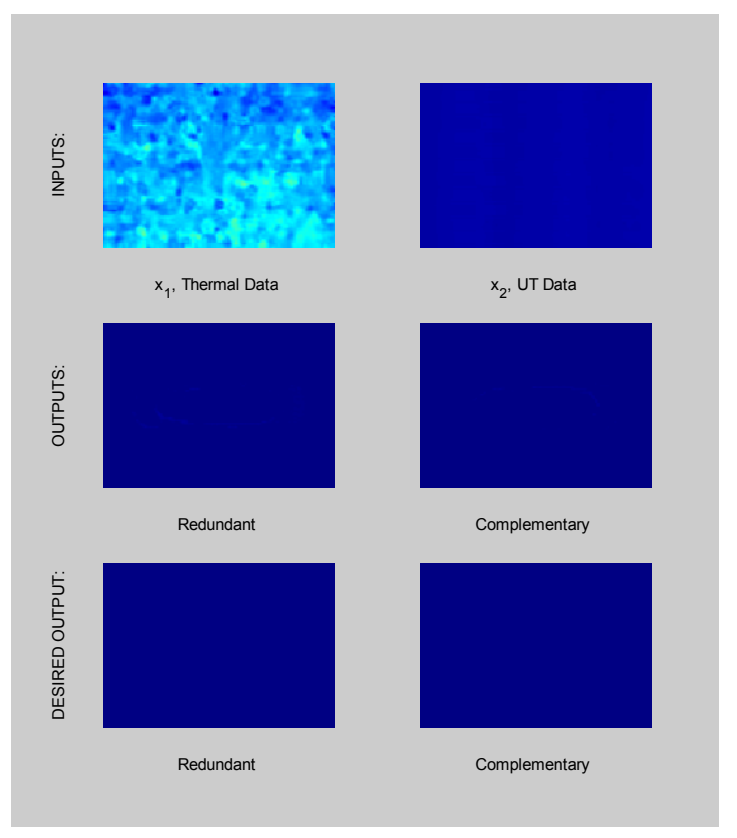

(s) Specimen 20b

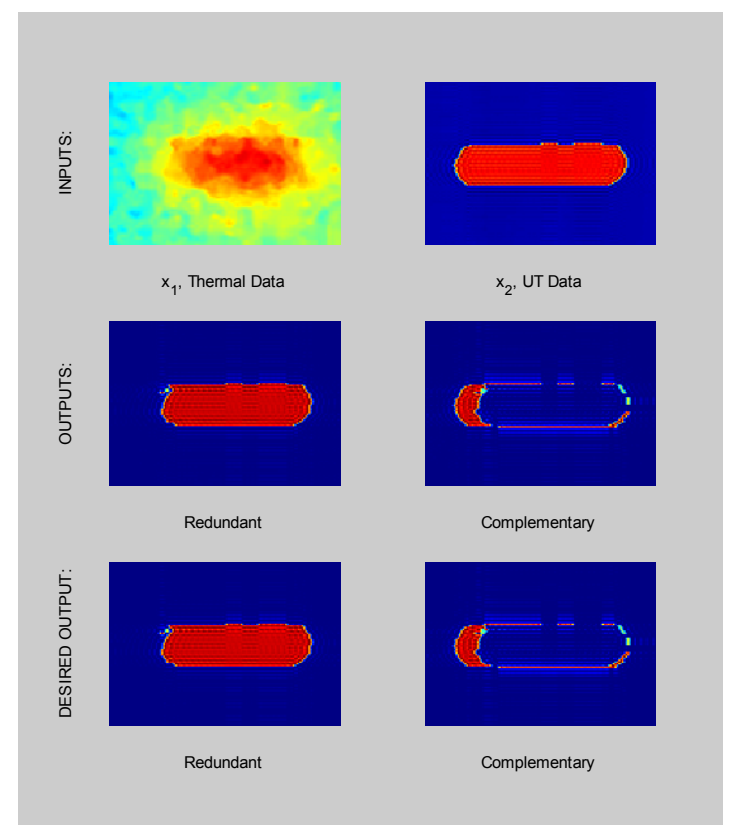

(r) Specimen 11b

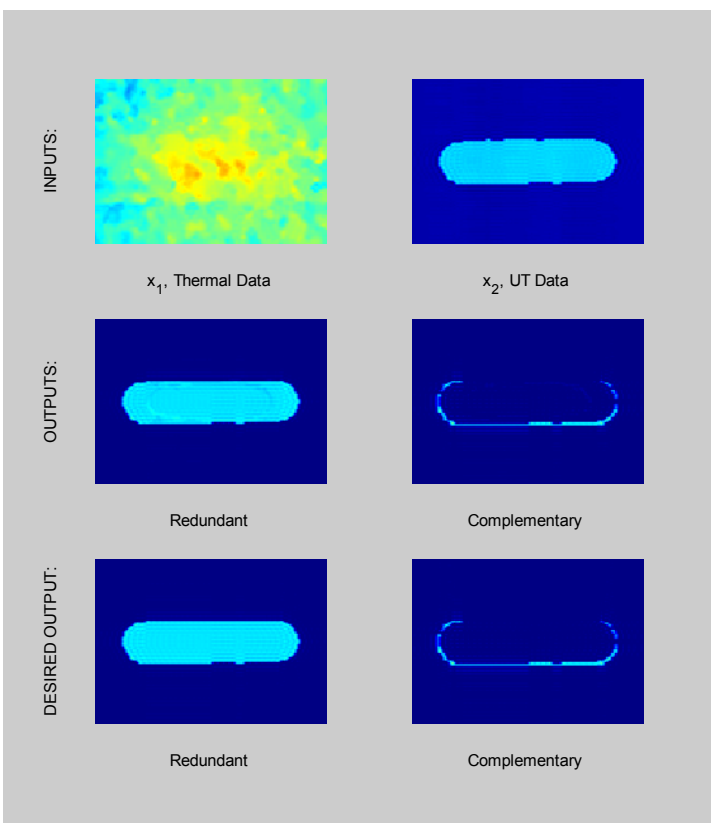

(t) Specimen 23b 
Trial 2: Thermal \& UT Results (cont.)

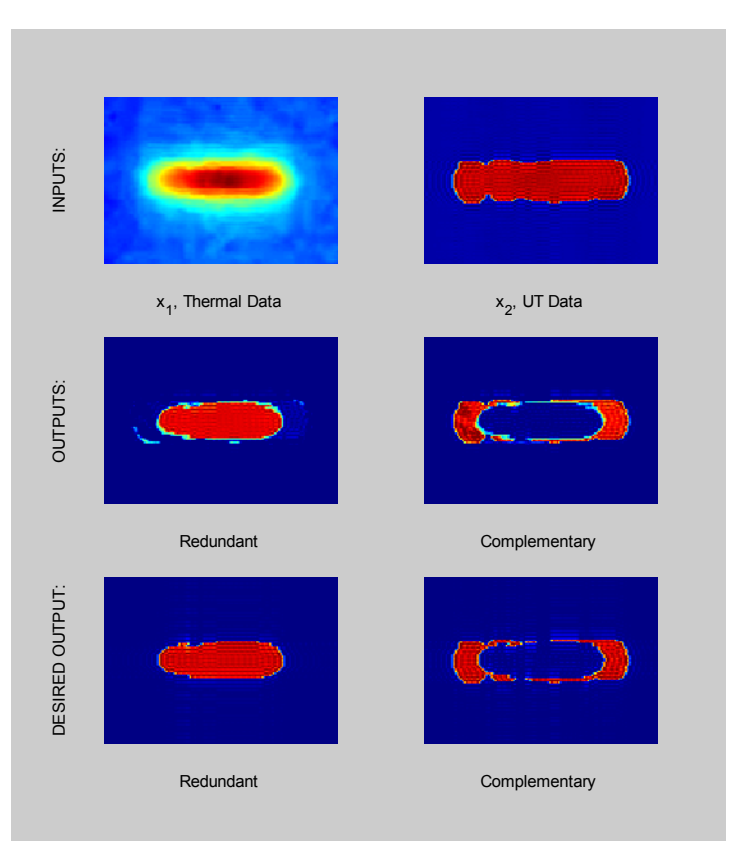

(u) Specimen 21b

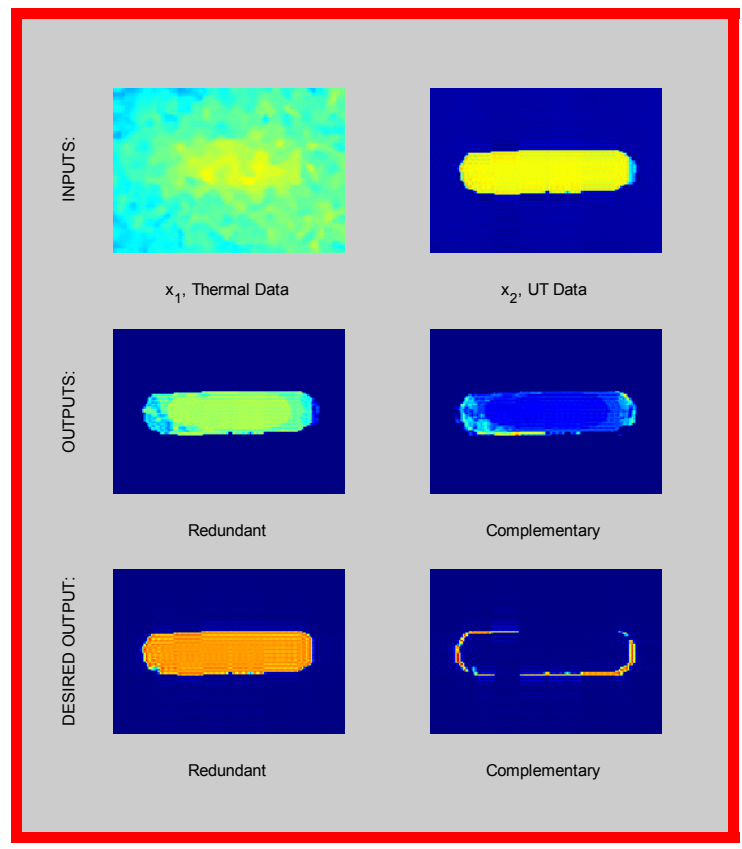

(w) Specimen 12b

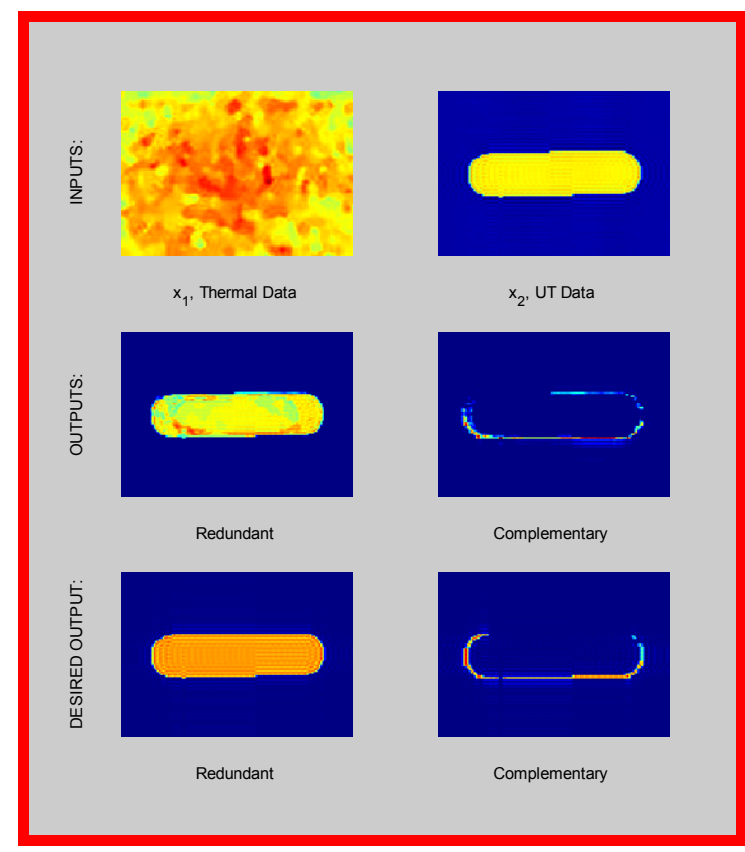

(v) Specimen 02b

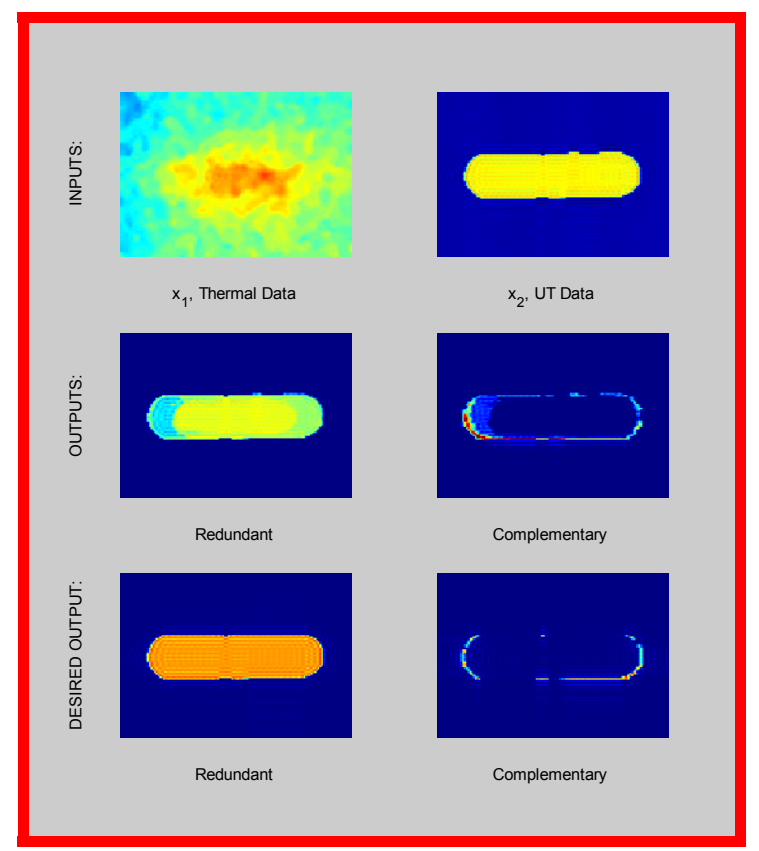

(x) Specimen 22b

Figure 6: Thermal \& UT combination Trial 2.

Training Data: (a) - (u); Test Data: (v), (w), (x) 
Trial 3: Thermal \& UT Results

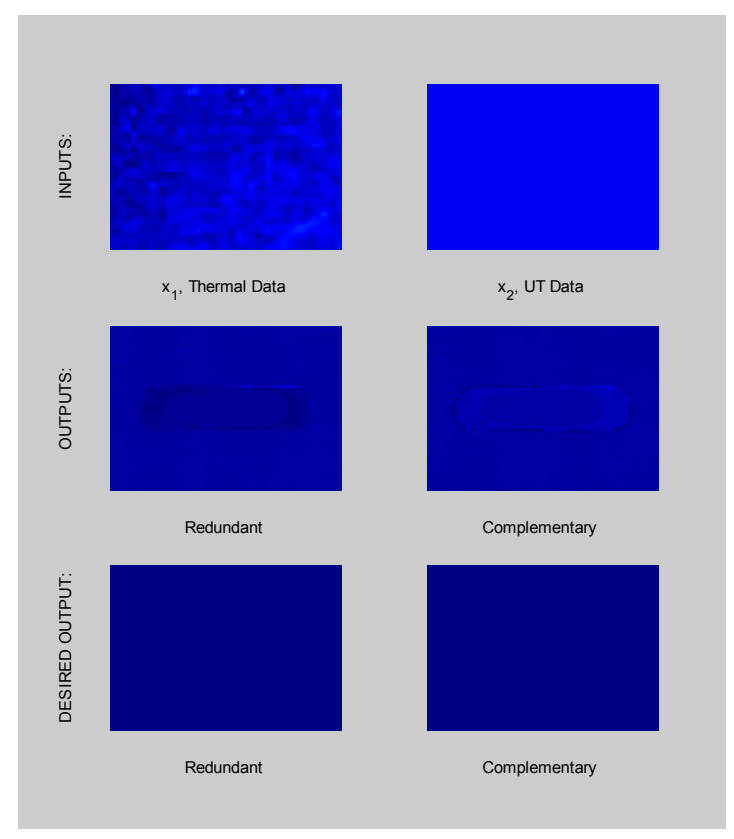

(a) Specimen 00a

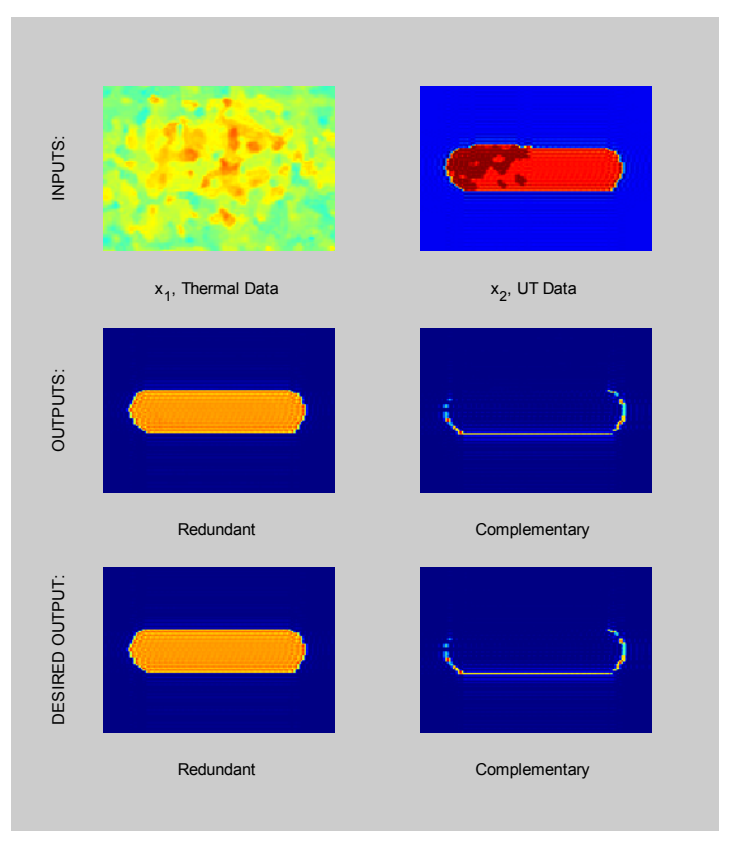

(c) Specimen 02a

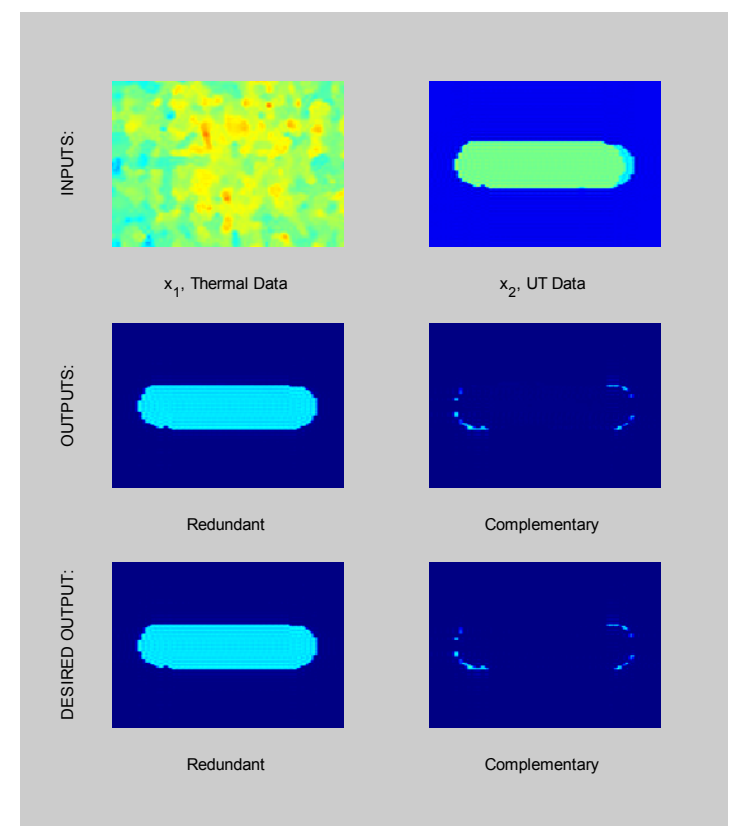

(b) Specimen 03a

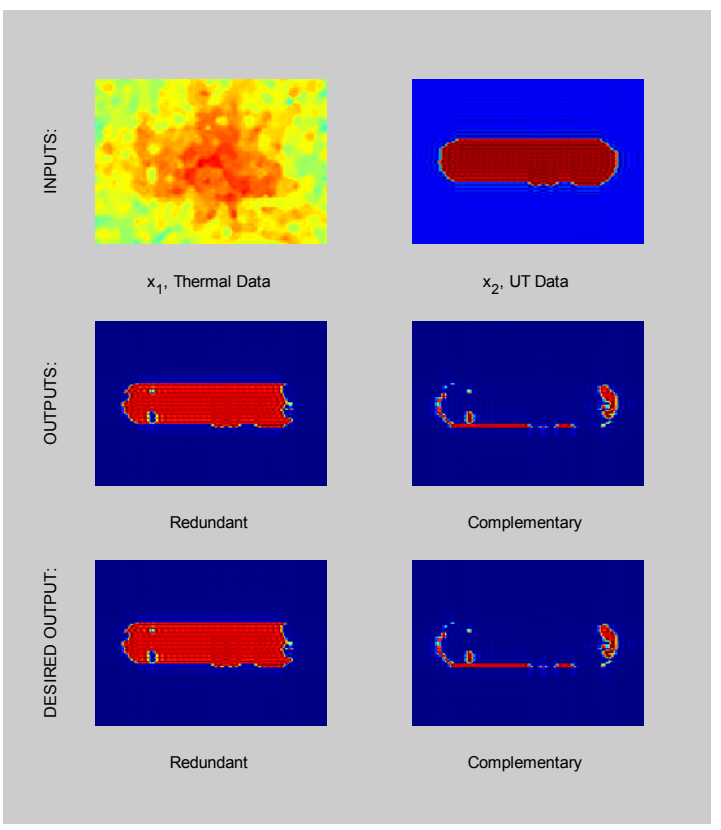

(d) Specimen 01a 
Trial 3: Thermal \& UT Results (cont.)

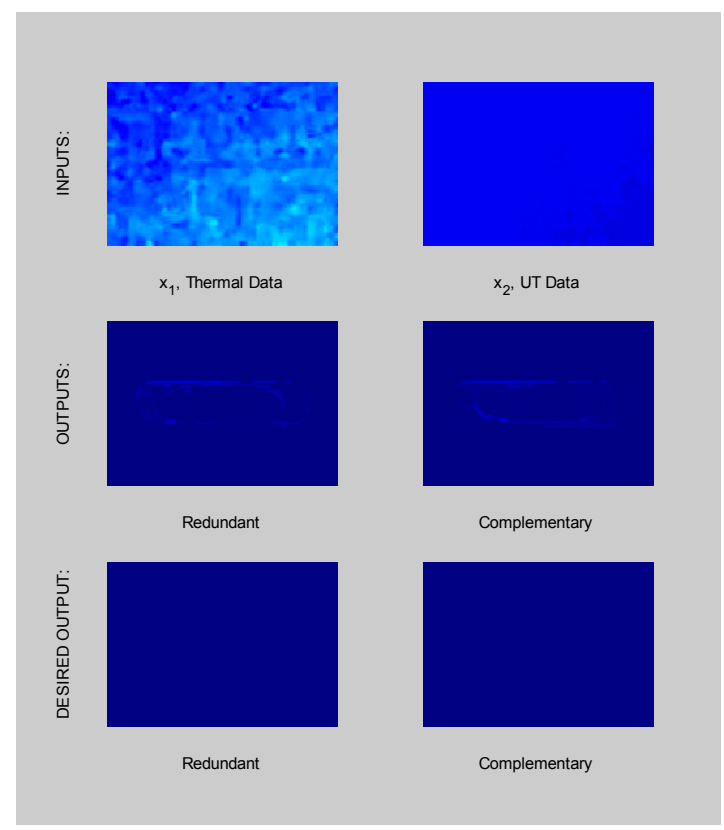

(e) Specimen 10a

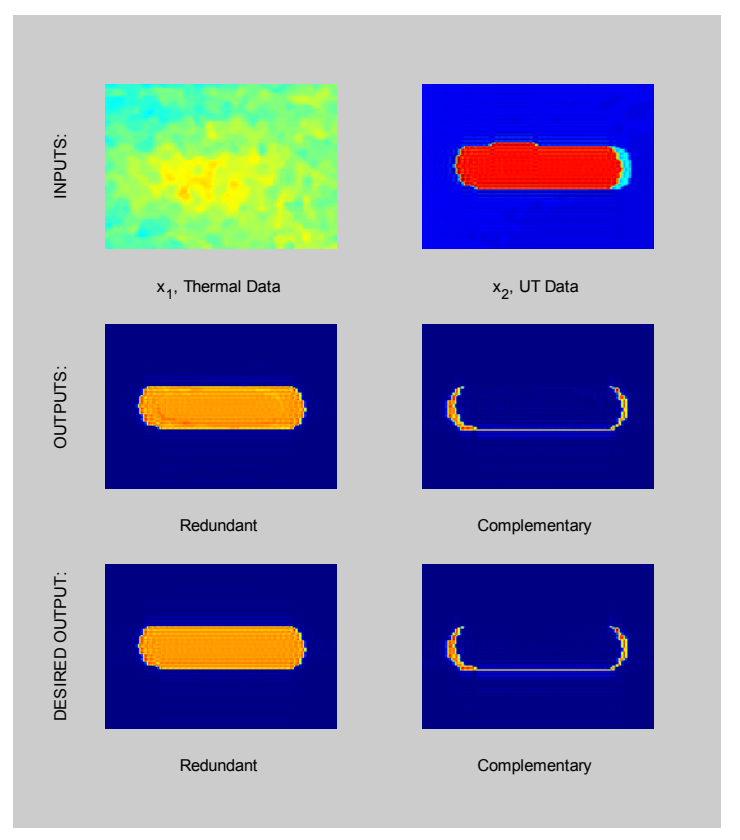

(g) Specimen 12a

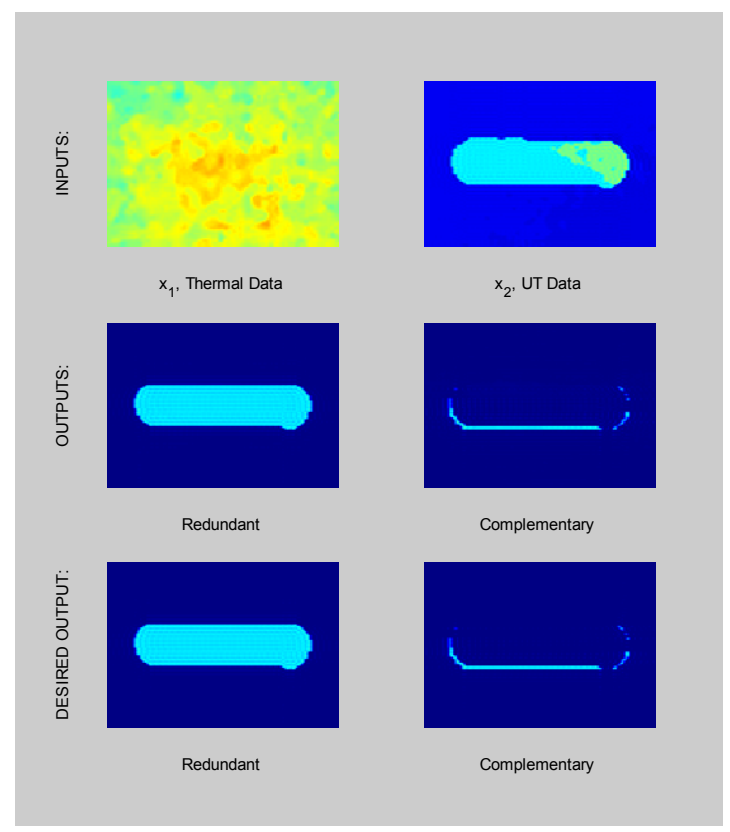

(f) Specimen 13a

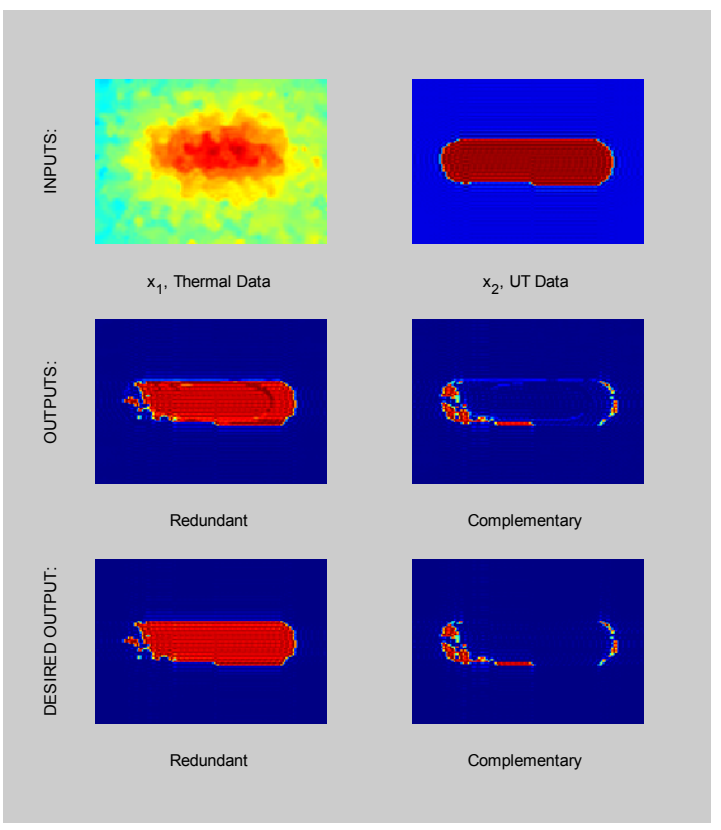

(h) Specimen 11a 
Trial 3: Thermal \& UT Results (cont.)

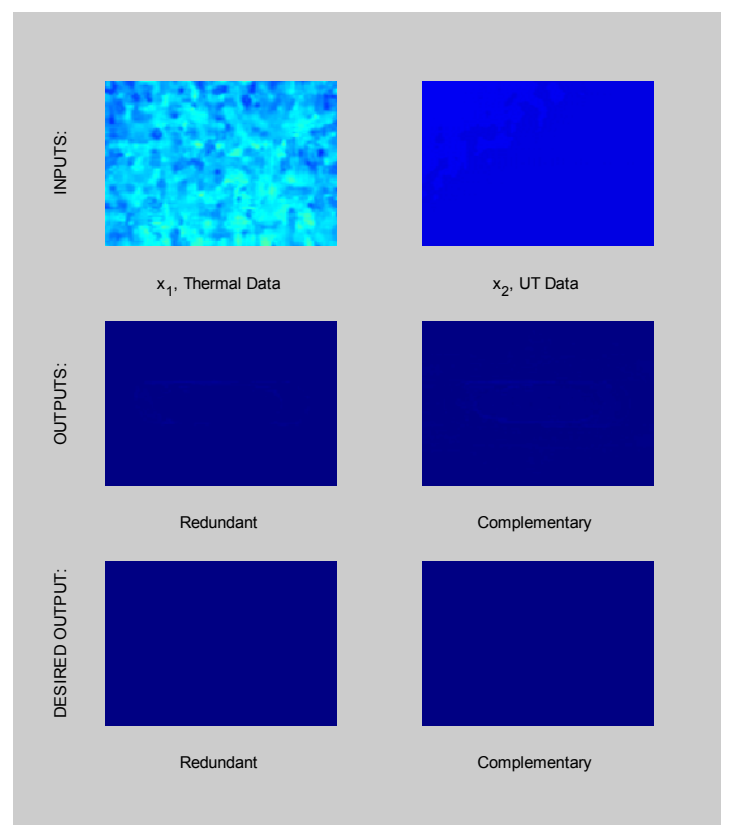

(i) Specimen 20a

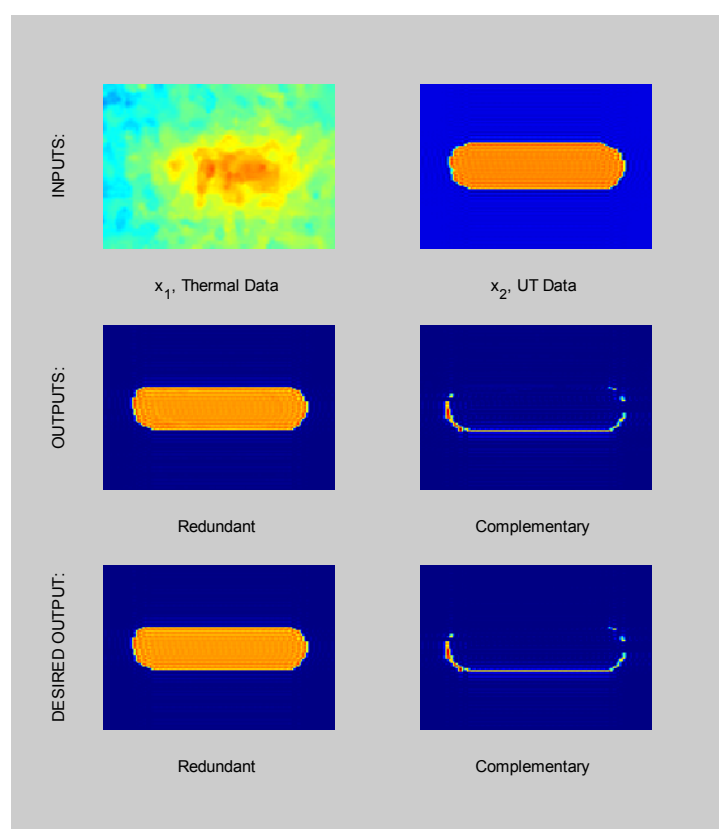

(k) Specimen 22a

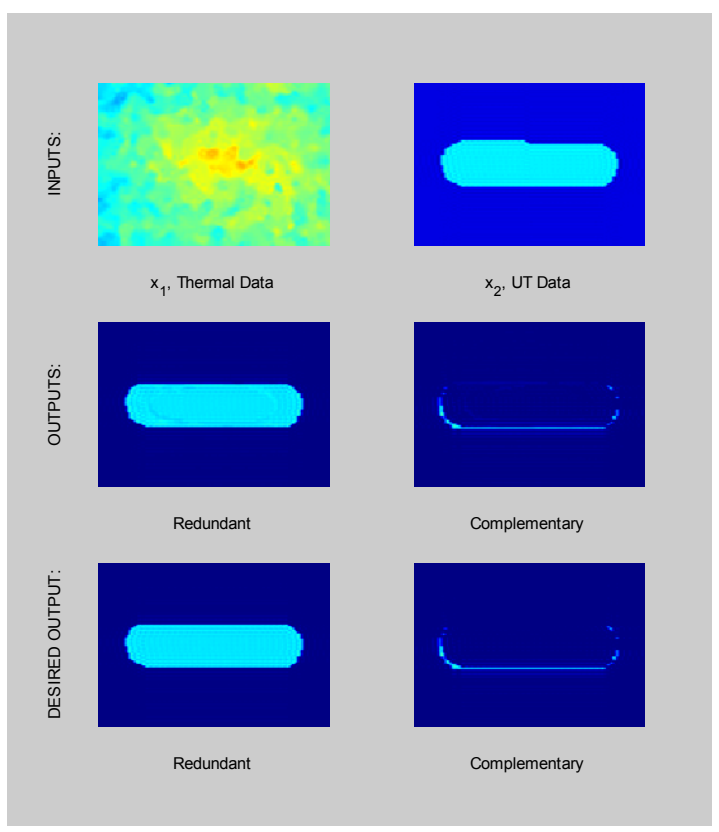

(j) Specimen 23a

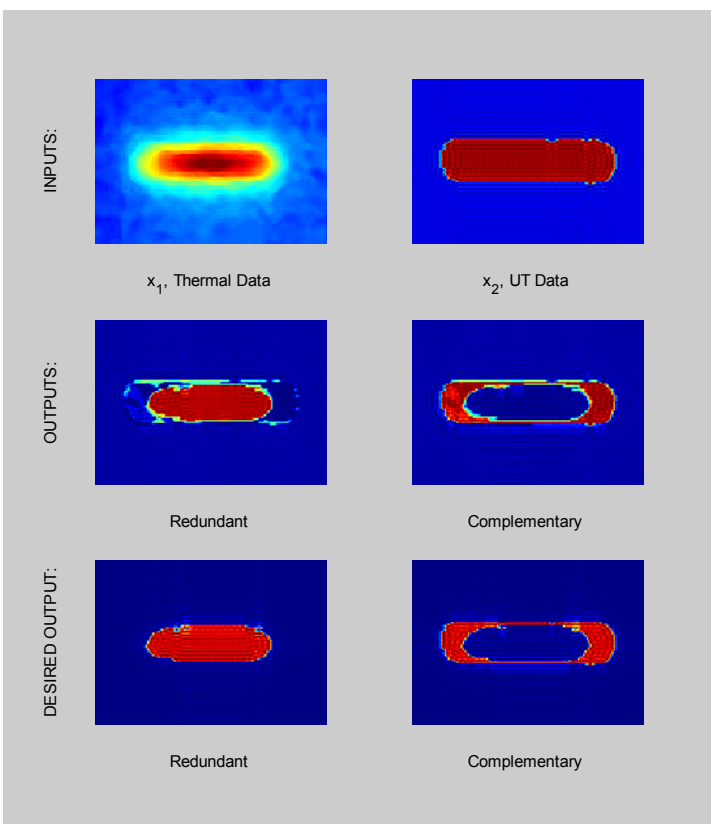

(l) Specimen 21a 
Trial 3: Thermal \& UT Results (cont.)

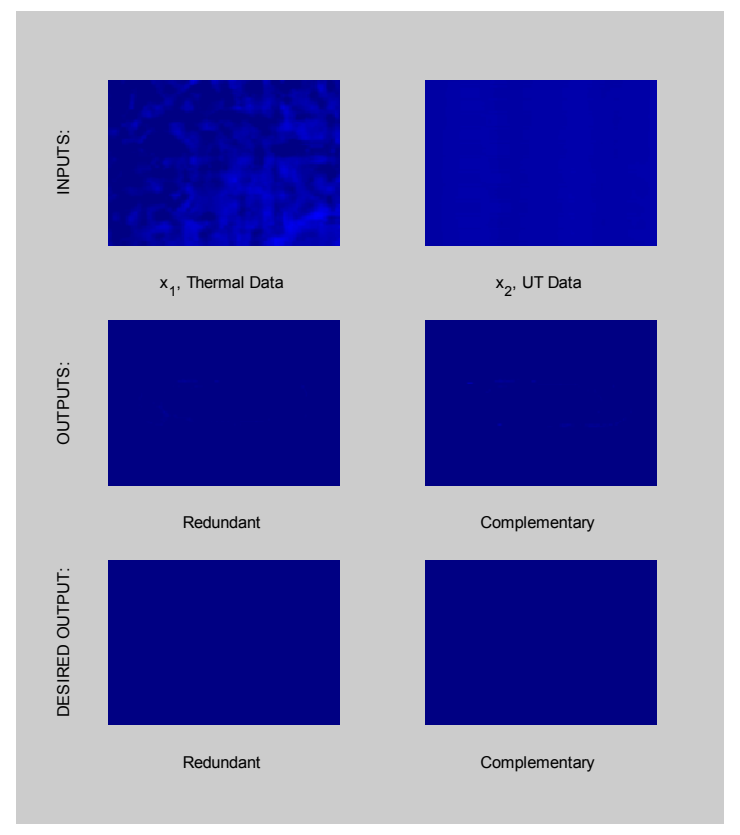

(m) Specimen 00b

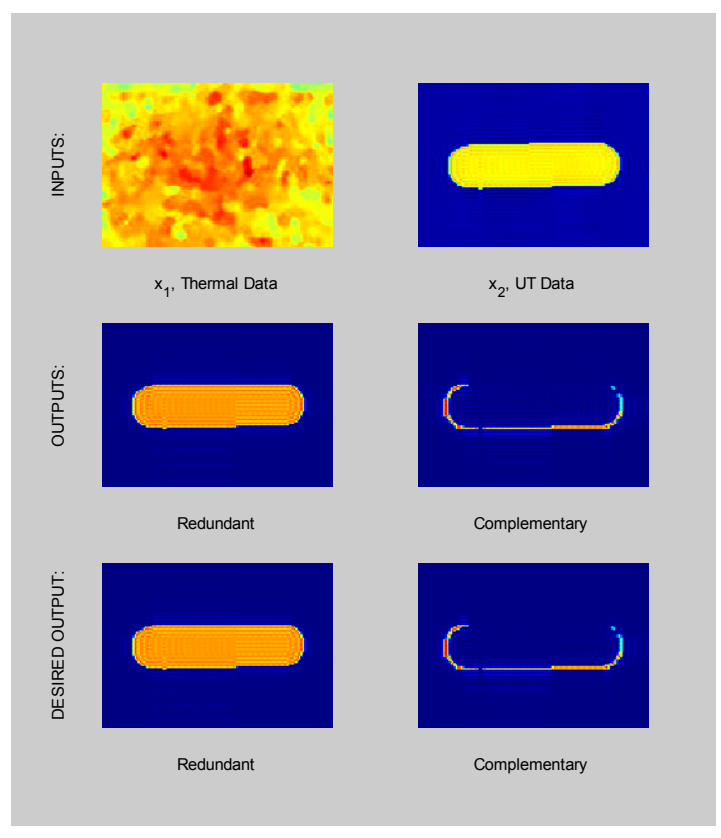

(o) Specimen 02b

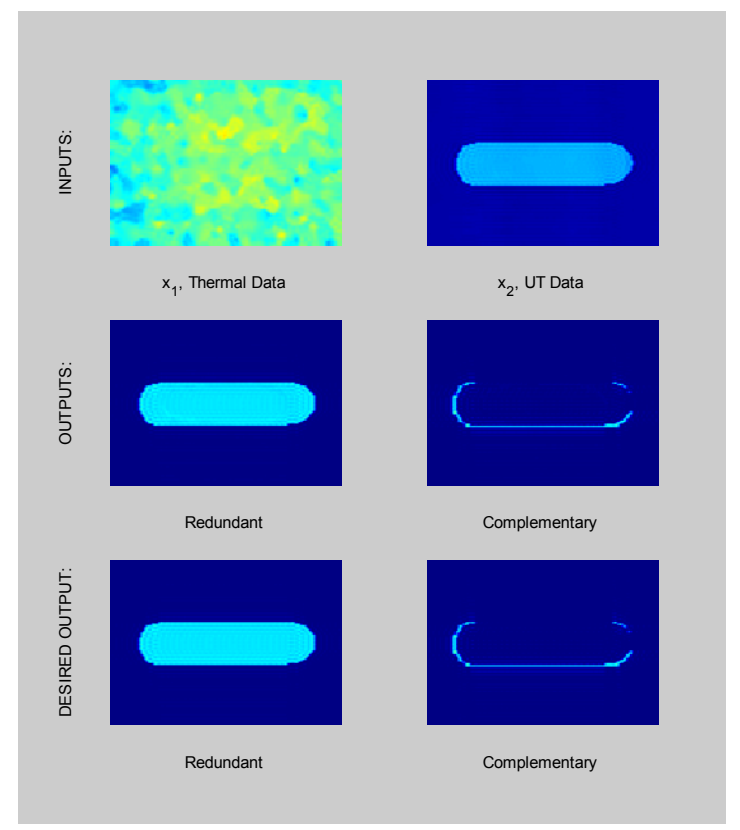

(n) Specimen 03b

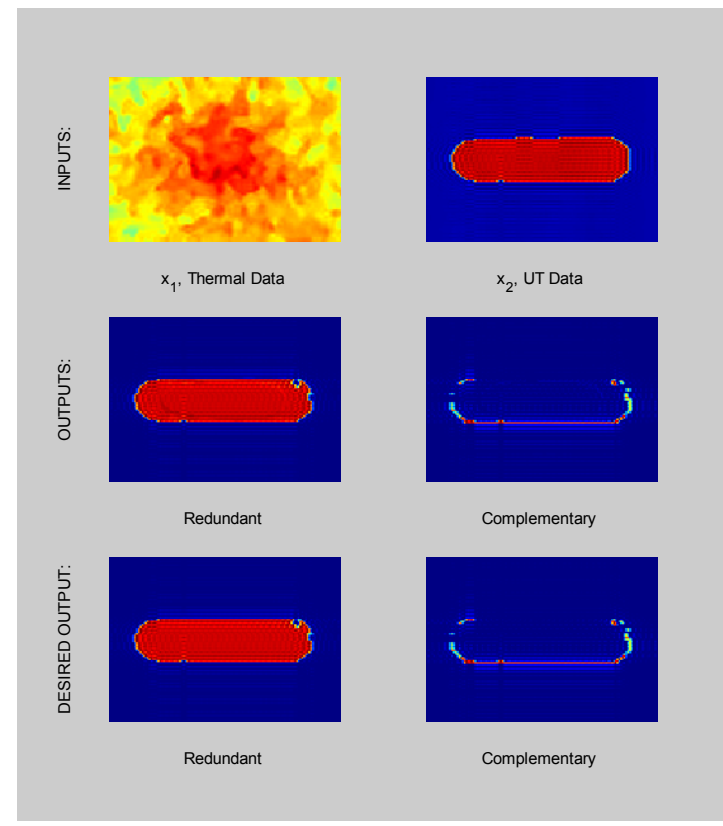

(p) Specimen 01b 
Trial 3: Thermal \& UT Results (cont.)

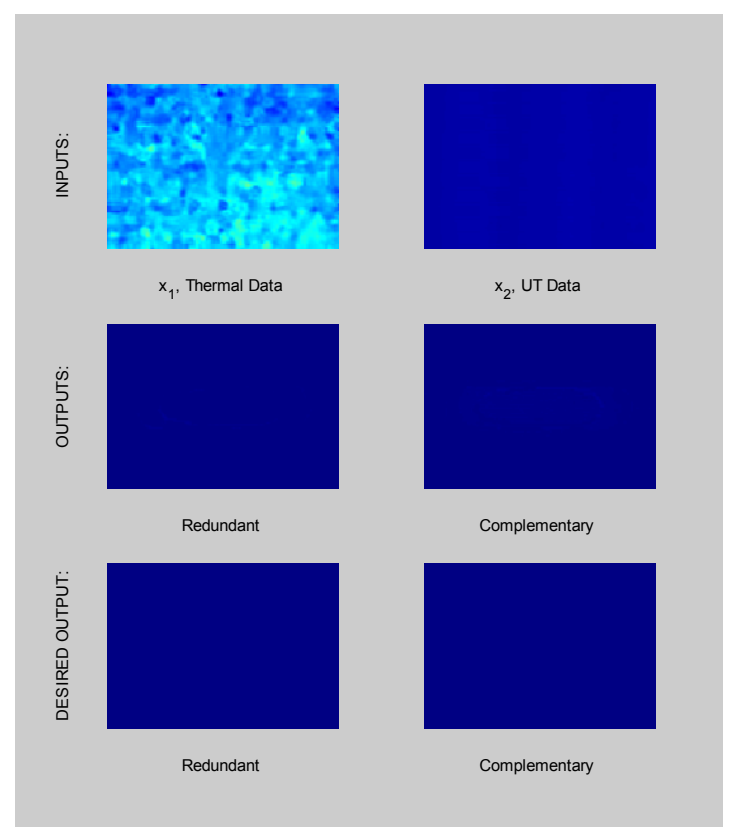

(q) Specimen 20b

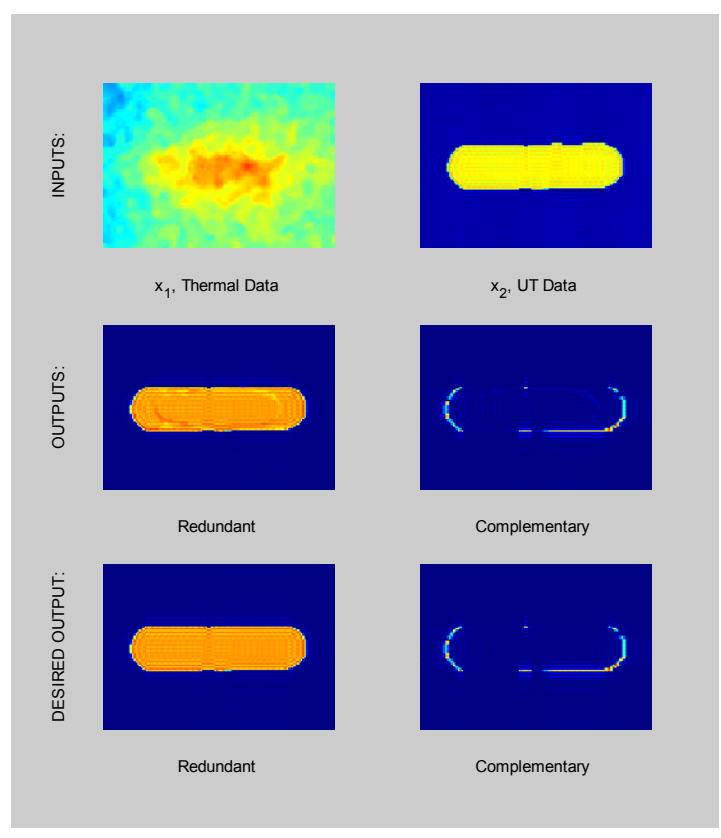

(s) Specimen 22b

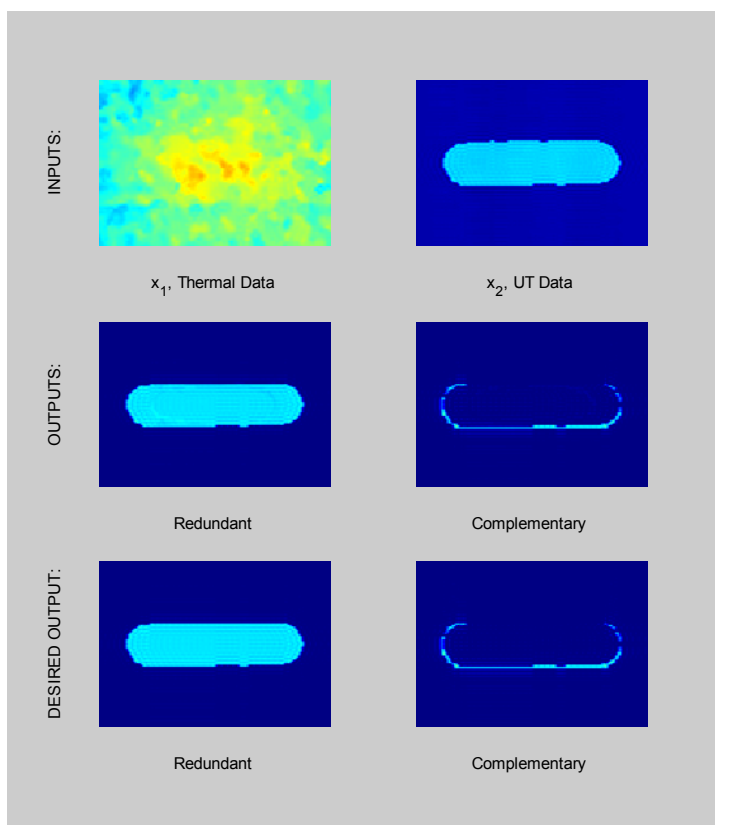

(r) Specimen 23b

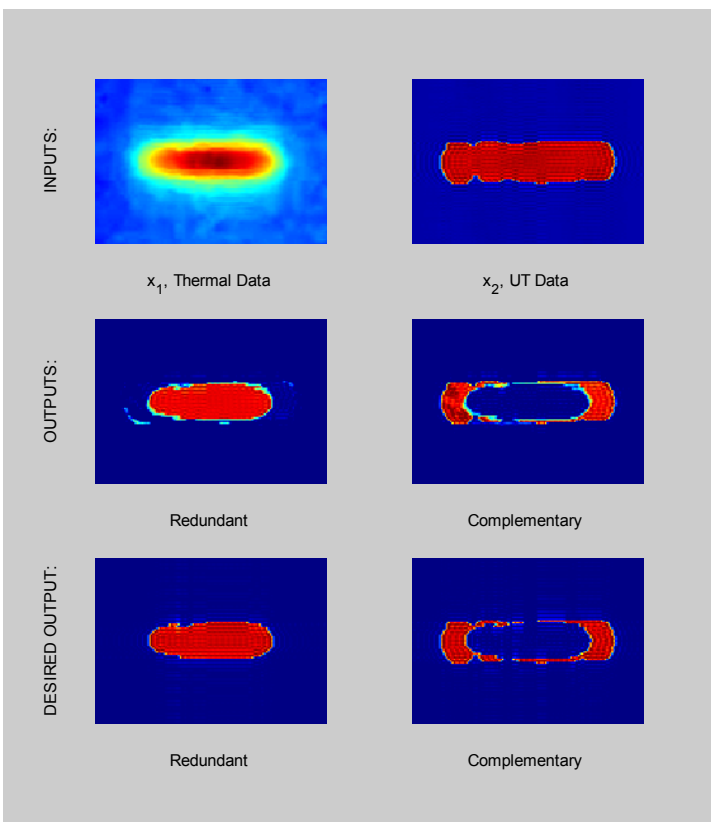

(t) Specimen 21b 
Trial 3: Thermal \& UT Results (cont.)

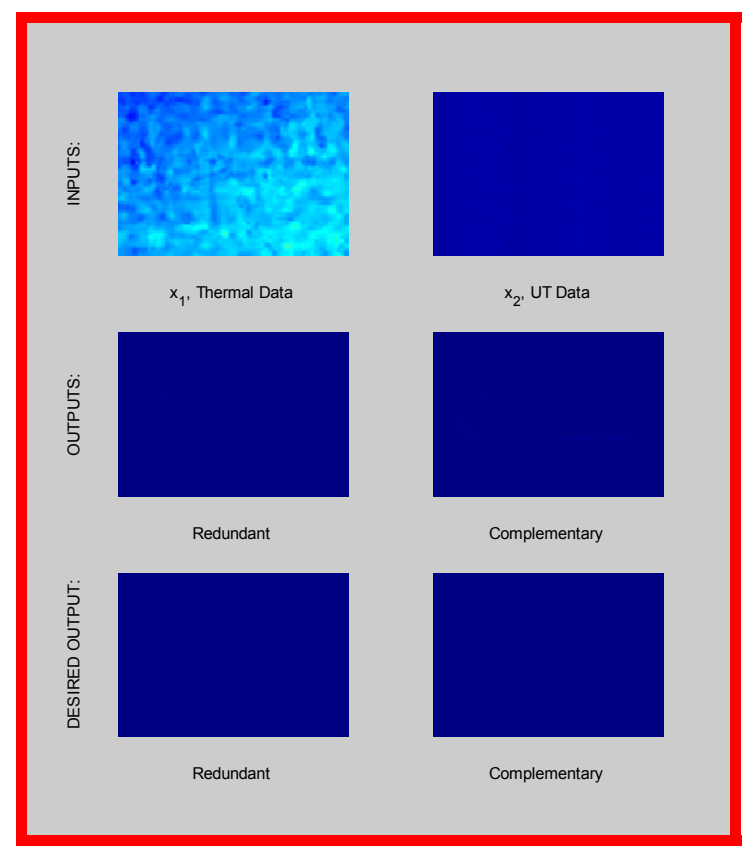

(u) Specimen 10b

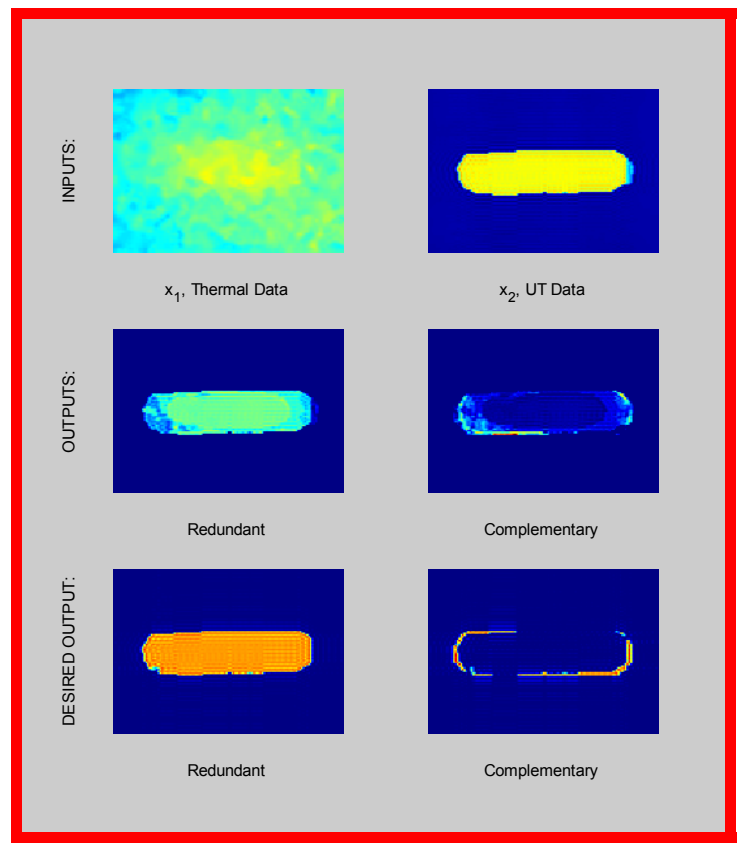

(w) Specimen 12b

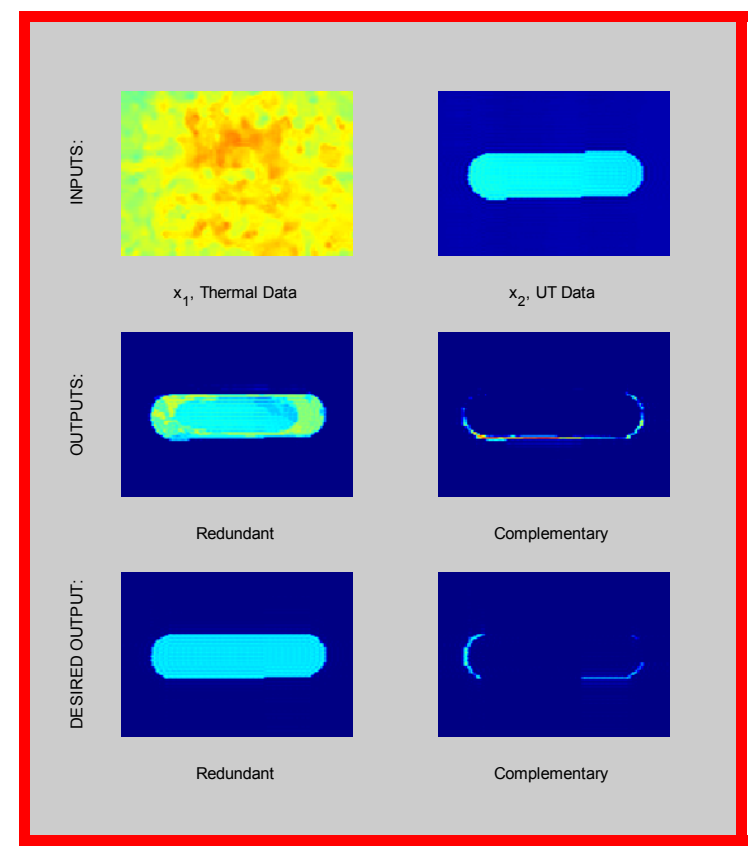

(v) Specimen 13b

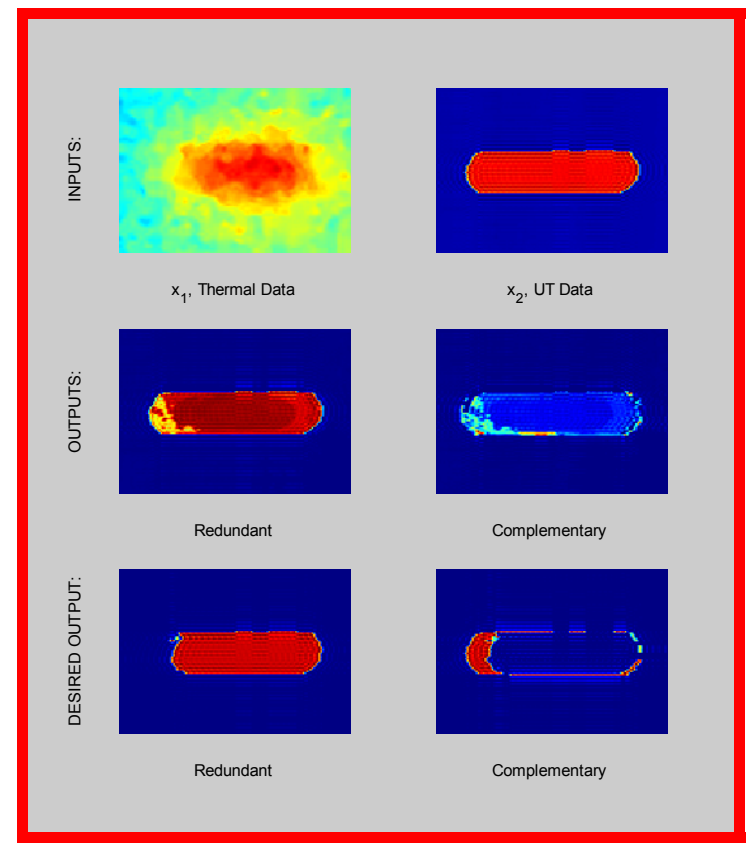

(x) Specimen 11b

Figure 7: Thermal \& UT combination Trial 3.

Training Data: (a) - (t); Test Data: (u), (v), (w), (x) 
MFL \& Thermal Data Fusion Results

Trial 1: MFL \& Thermal Results

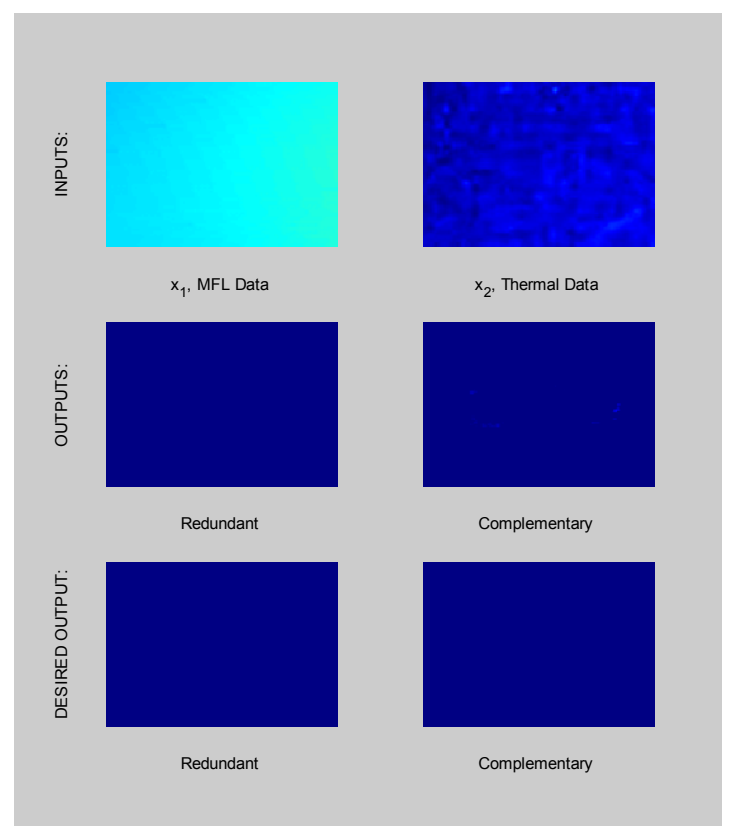

(a) Specimen 00a

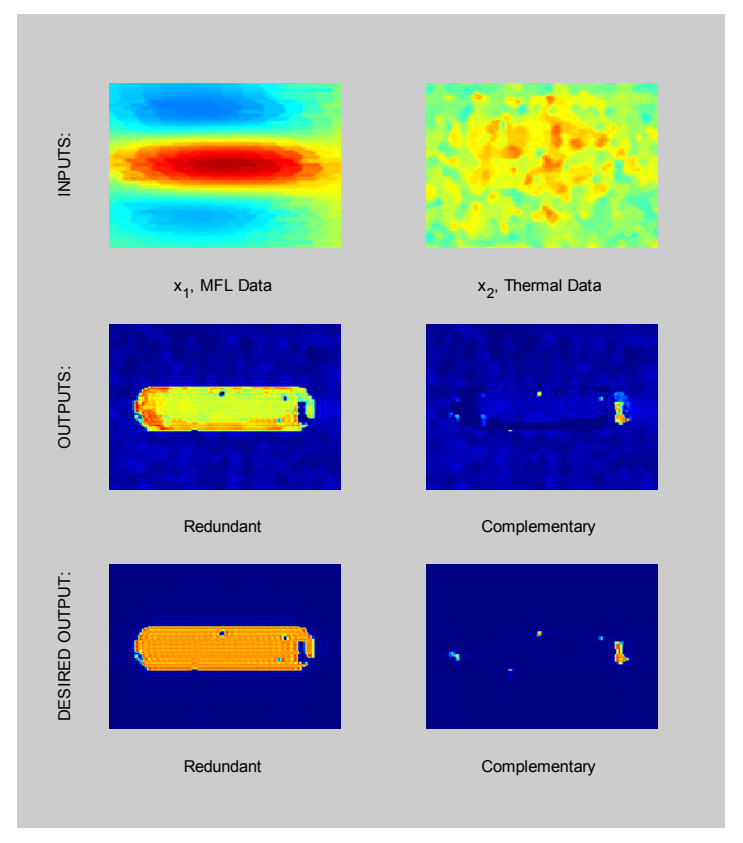

(c) Specimen 02a

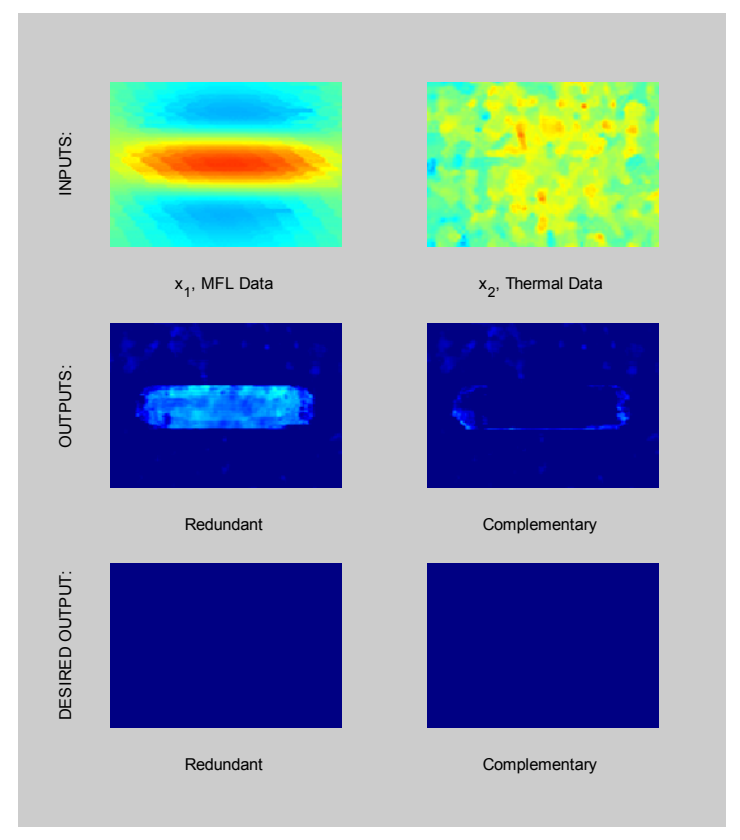

(b) Specimen 03a

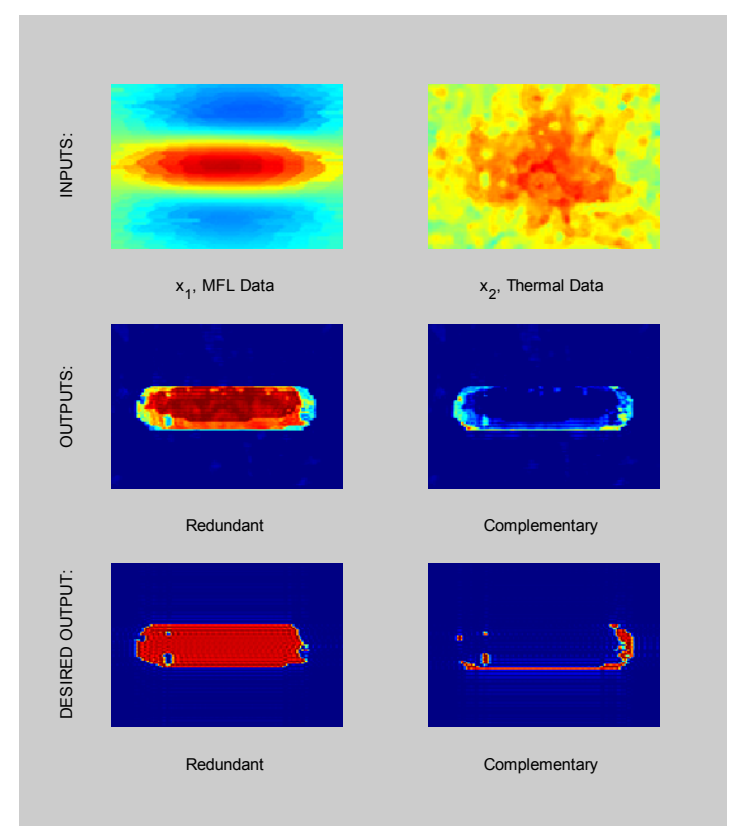

(d) Specimen 01a 
Trial 1: MFL \& Thermal Results (cont.)

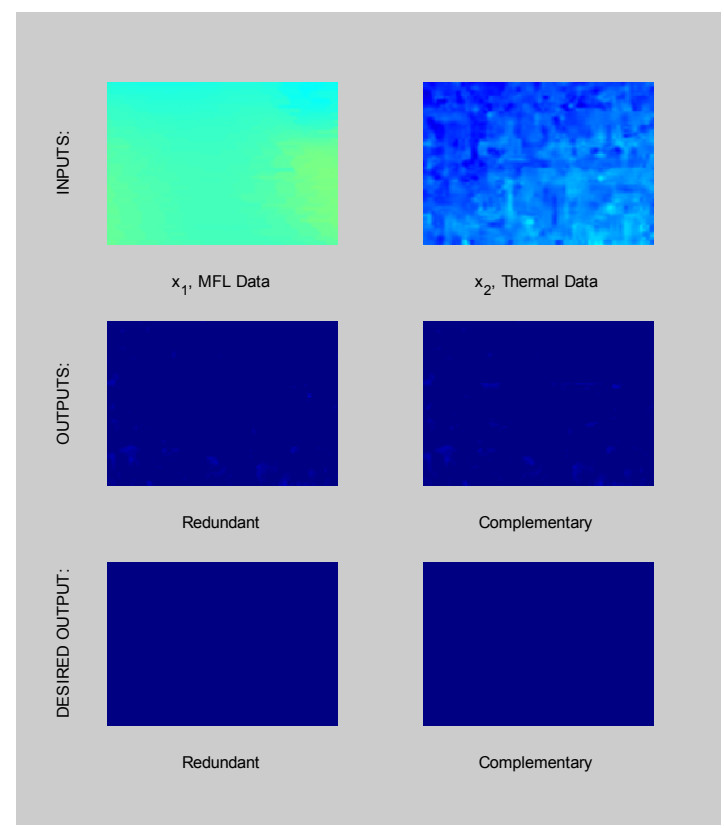

(e) Specimen 10a

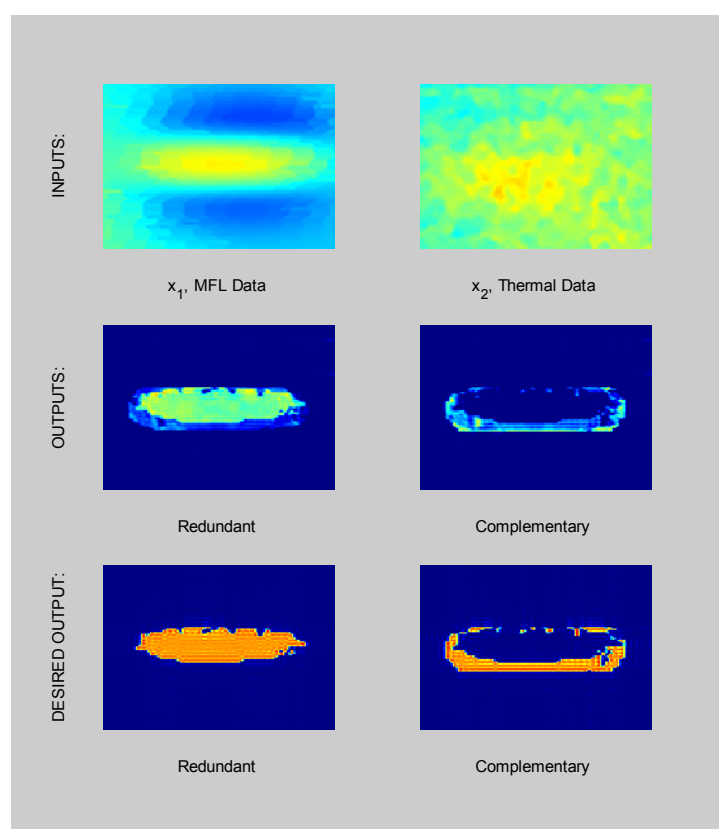

(g) Specimen 12a

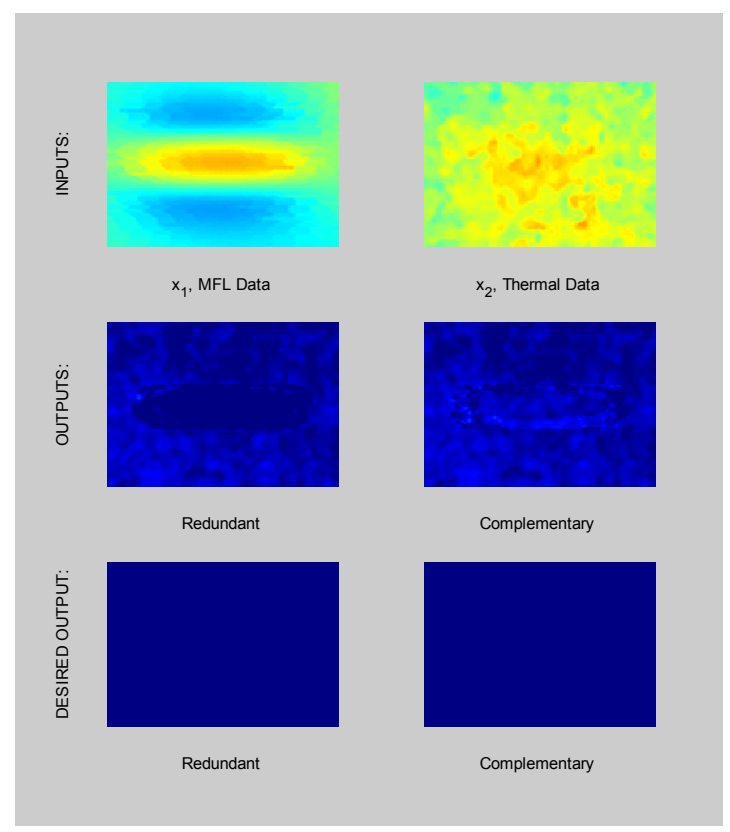

(f) Specimen 13a

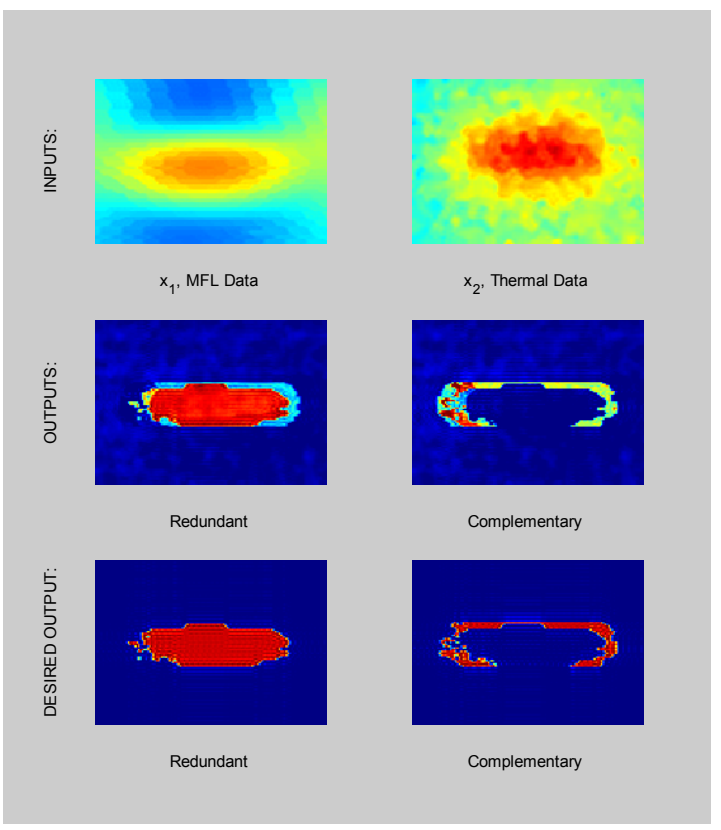

(h) Specimen 11a 
Trial 1: MFL \& Thermal Results (cont.)

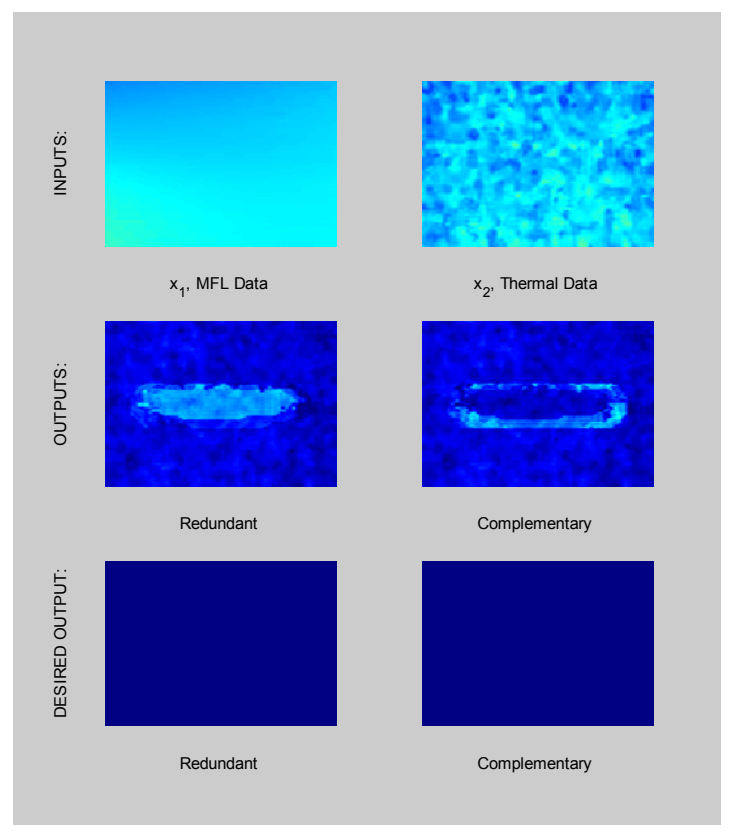

(i) Specimen 20a

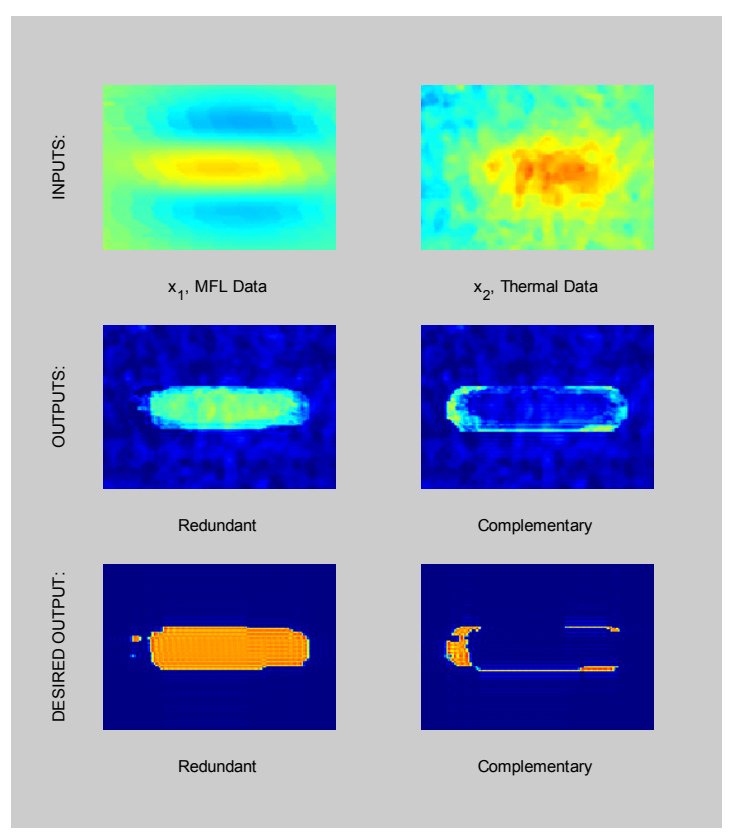

(k) Specimen 22a

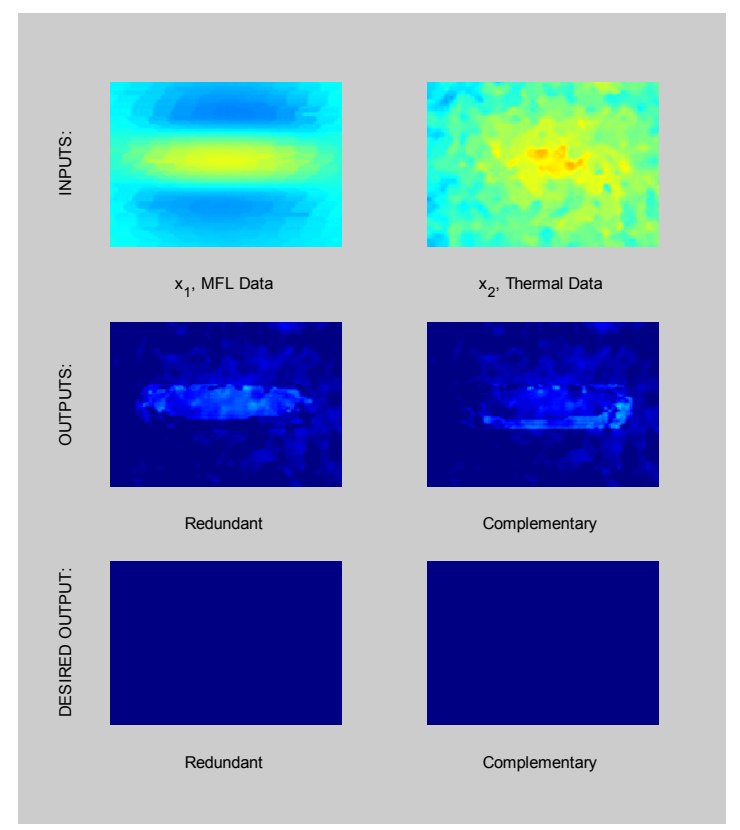

(j) Specimen 23a

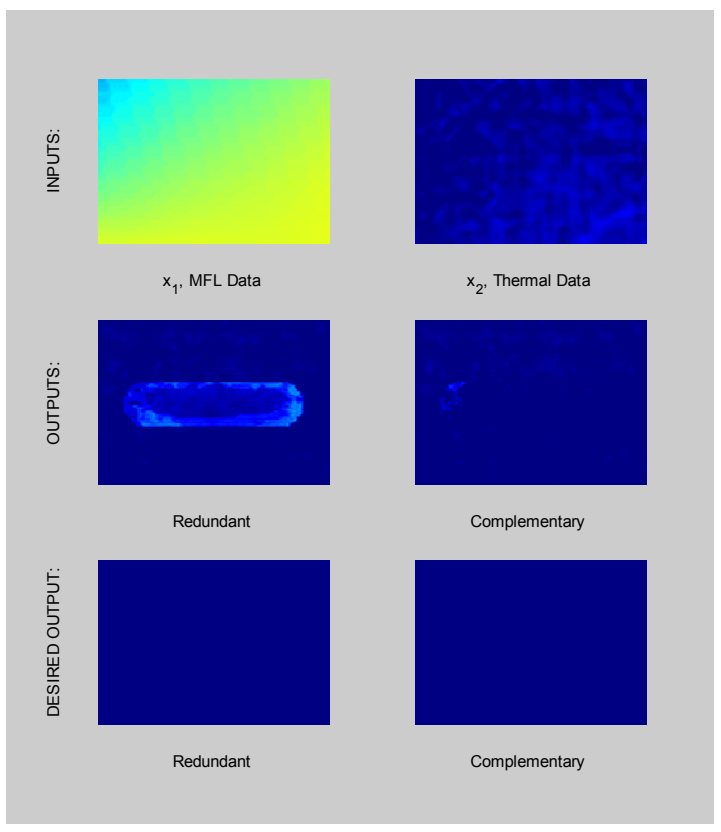

(l) Specimen 00b 
Trial 1: MFL \& Thermal Results (cont.)

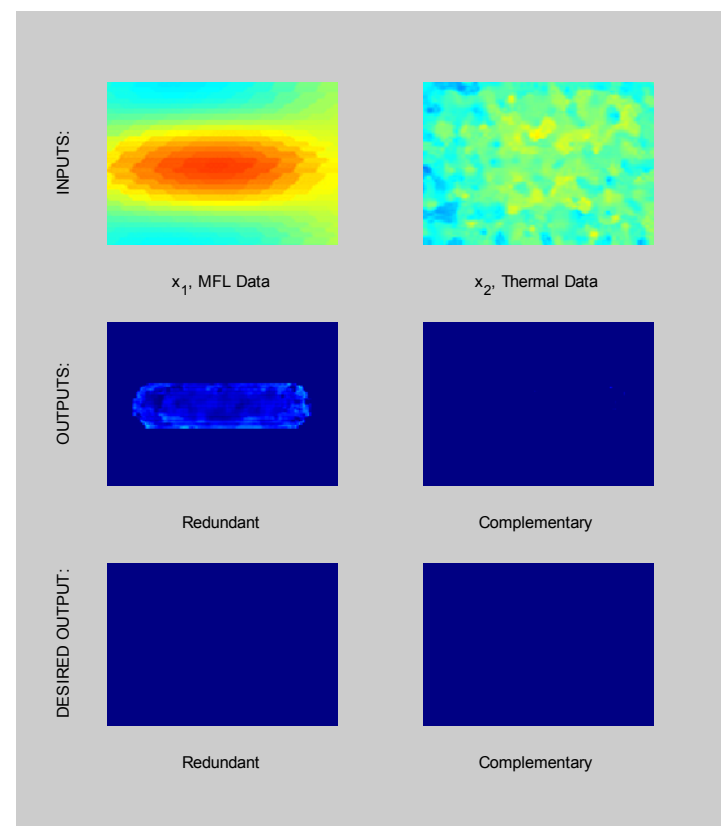

(m) Specimen 03b

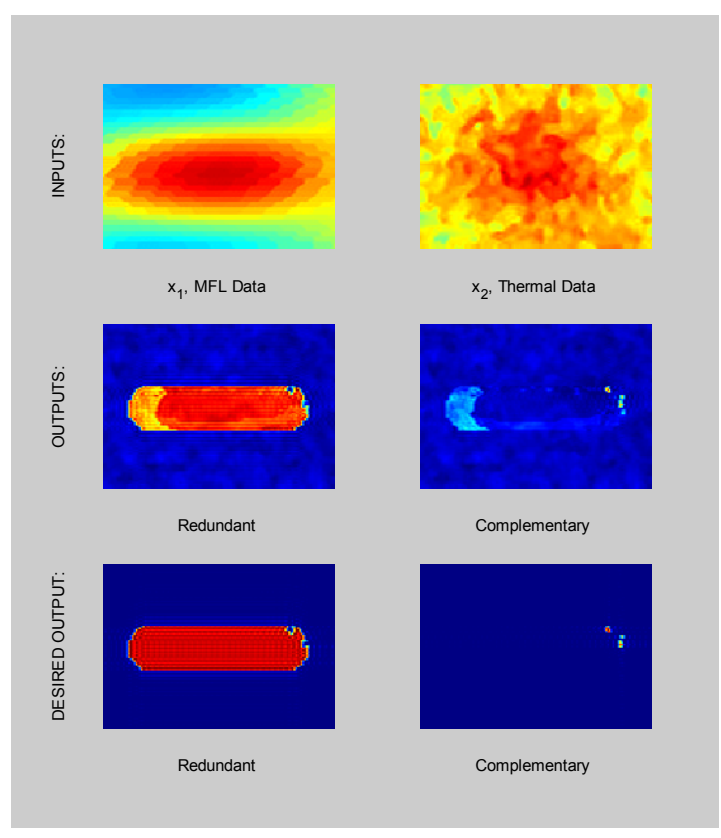

(o) Specimen 01b

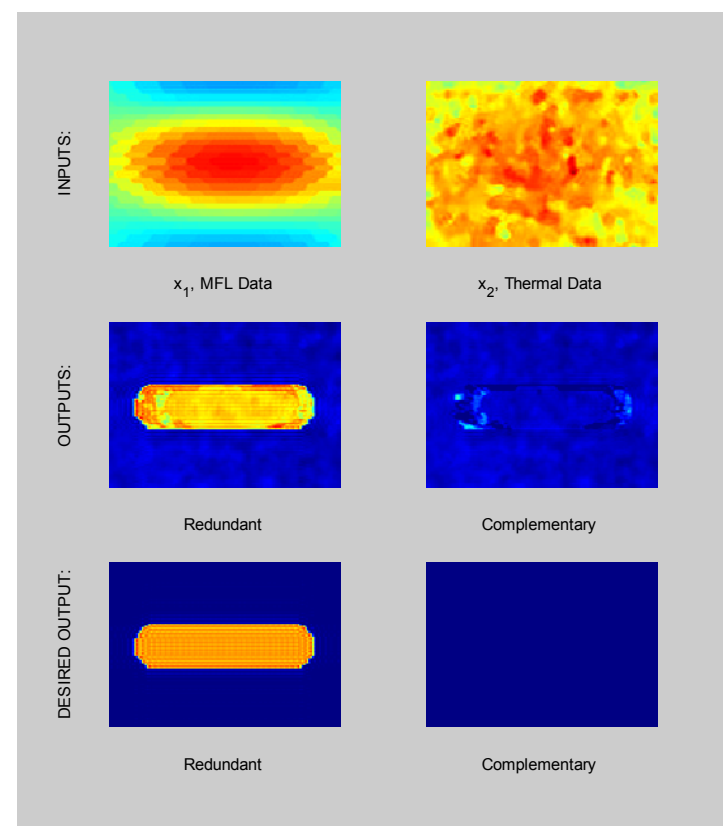

(n) Specimen 02b

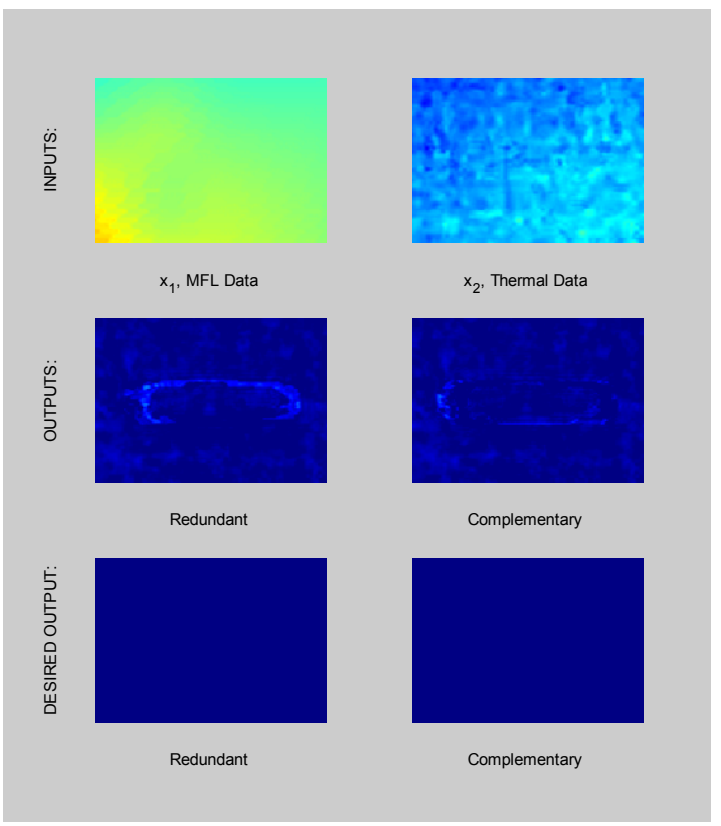

(p) Specimen 10b 
Trial 1: MFL \& Thermal Results (cont.)

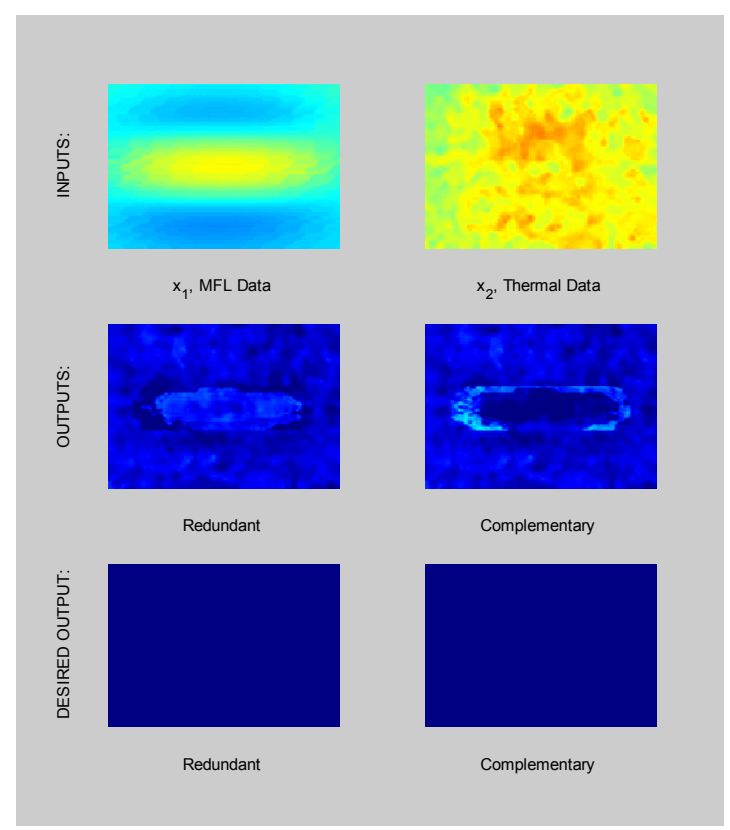

(q) Specimen 13b

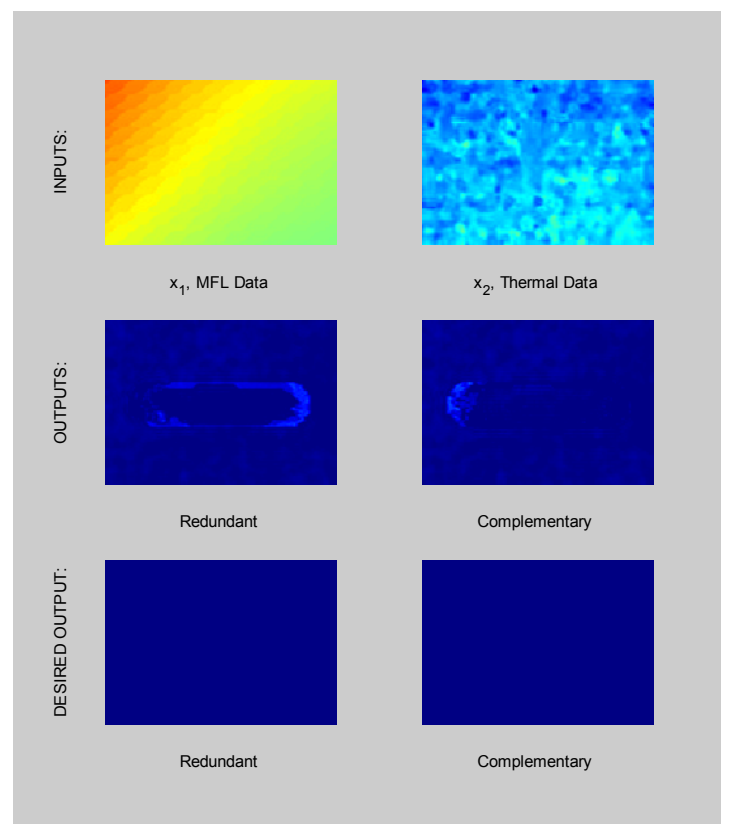

(s) Specimen 20b

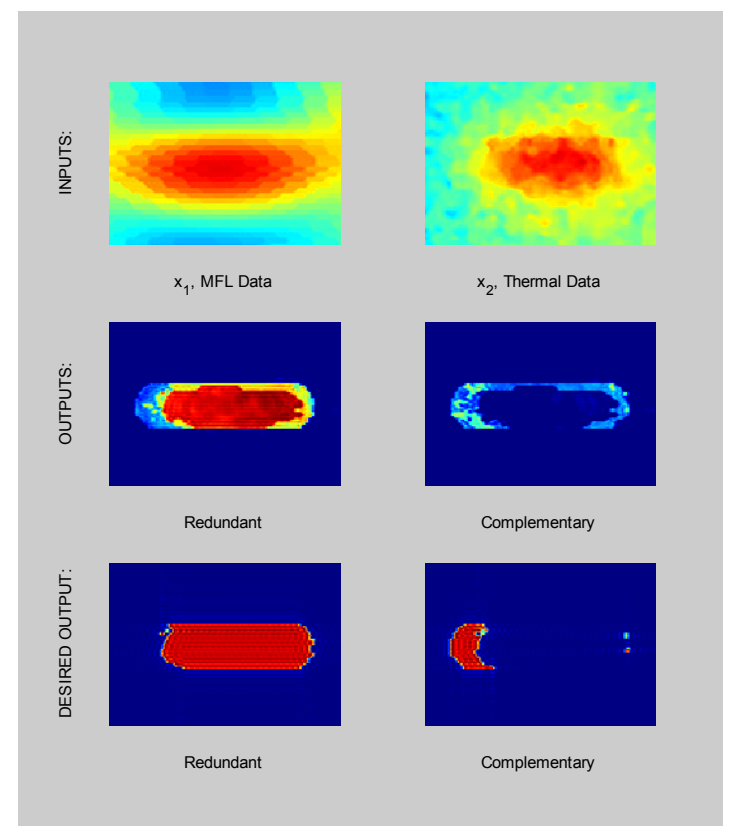

(r) Specimen 11b

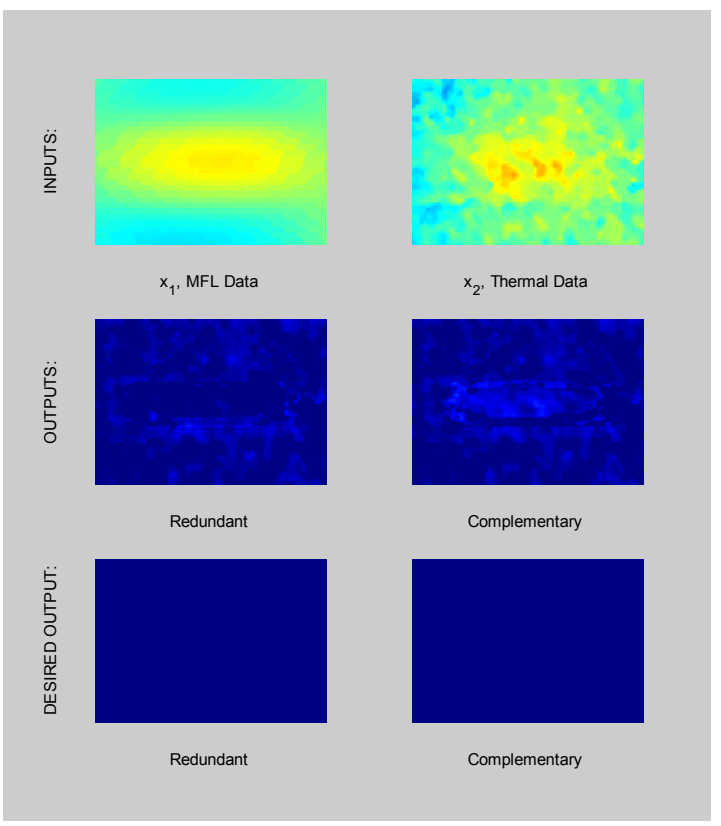

(t) Specimen 23b 
Trial 1: MFL \& Thermal Results (cont.)

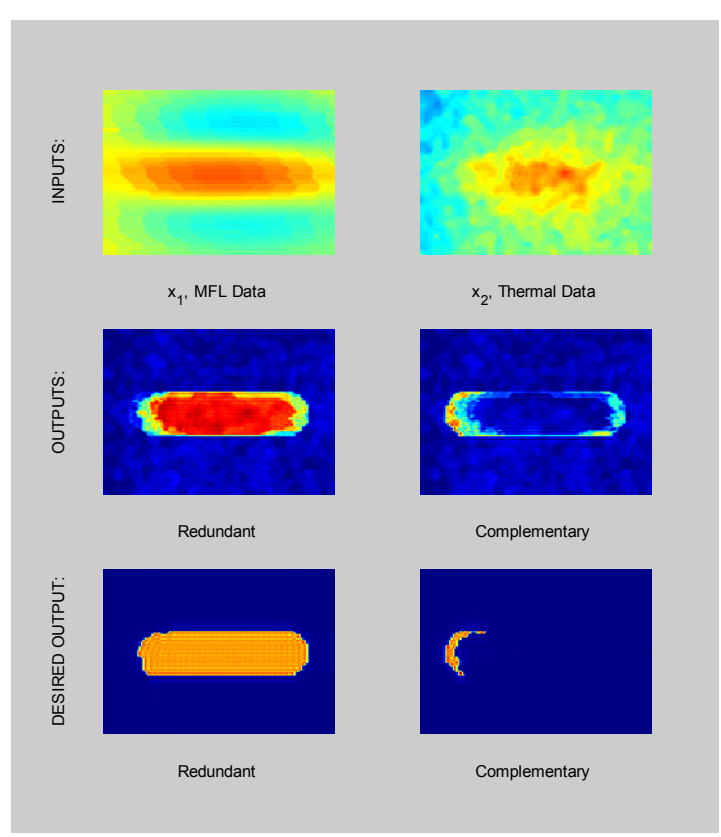

(u) Specimen 21b

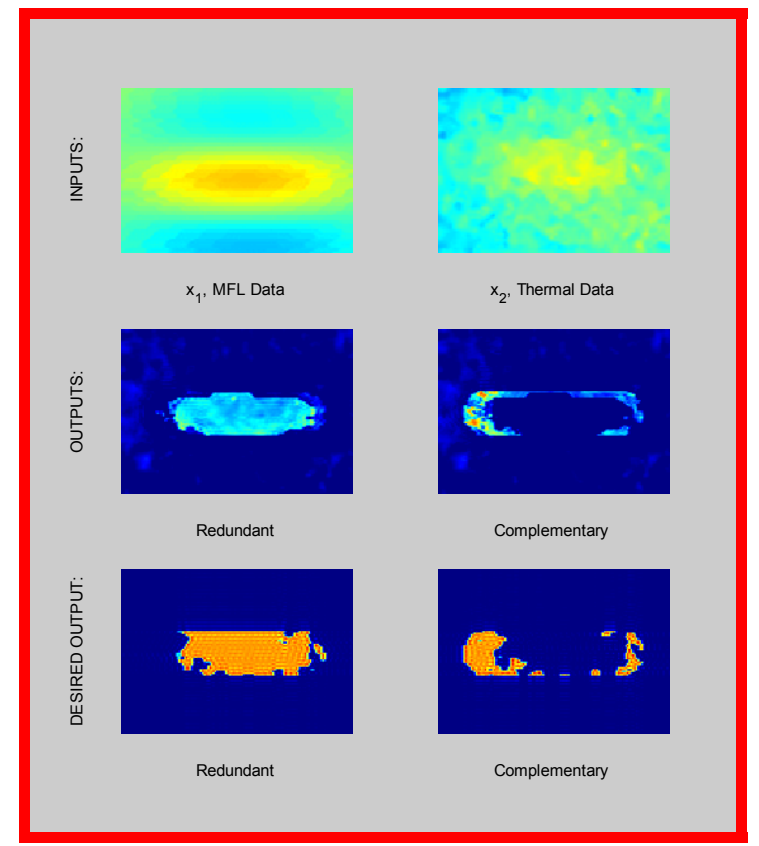

(v) Specimen 12b

Figure 8: MFL \& Thermal combination Trial 1.

Training Data: (a) - (u); Test Data: (v) 
Trial 2: MFL \& Thermal Results

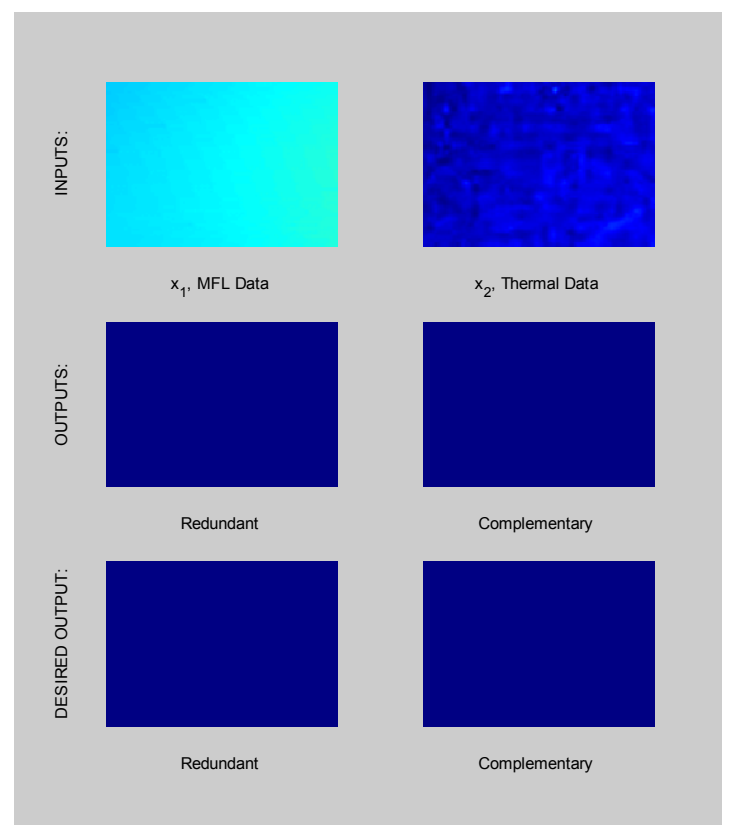

(a) Specimen 00a

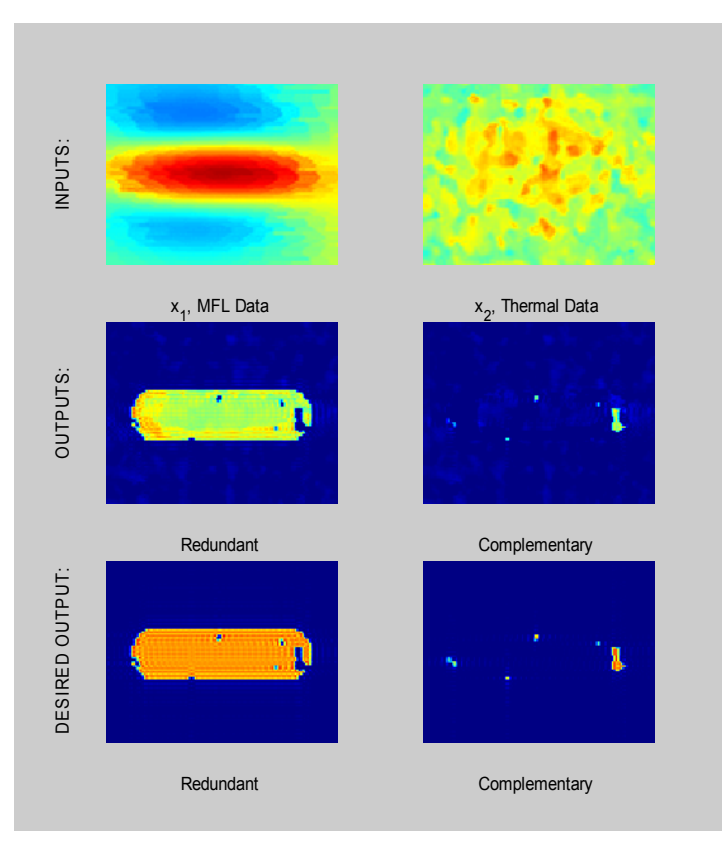

(c) Specimen 02a

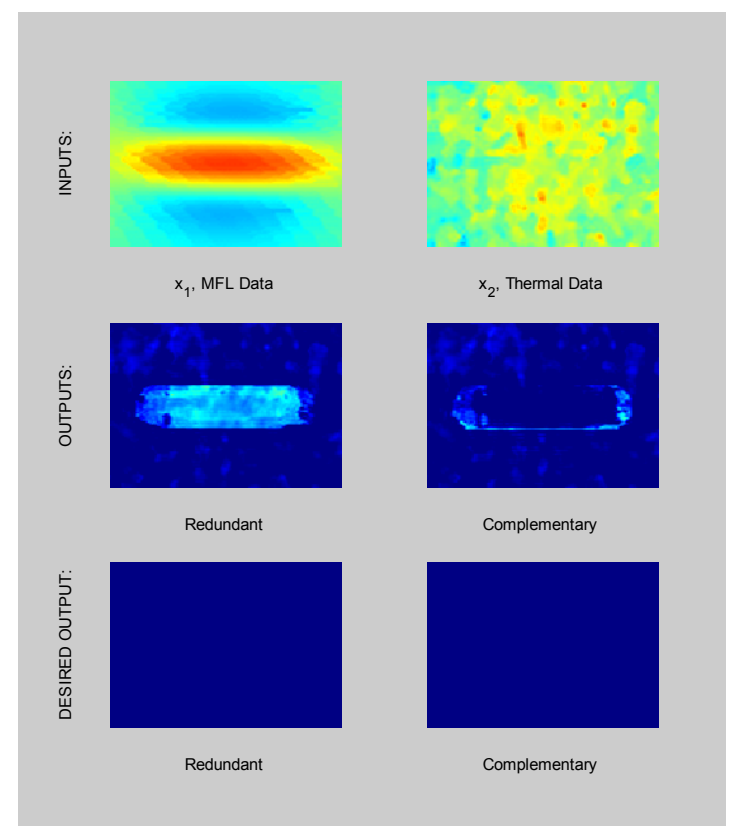

(b) Specimen 03a

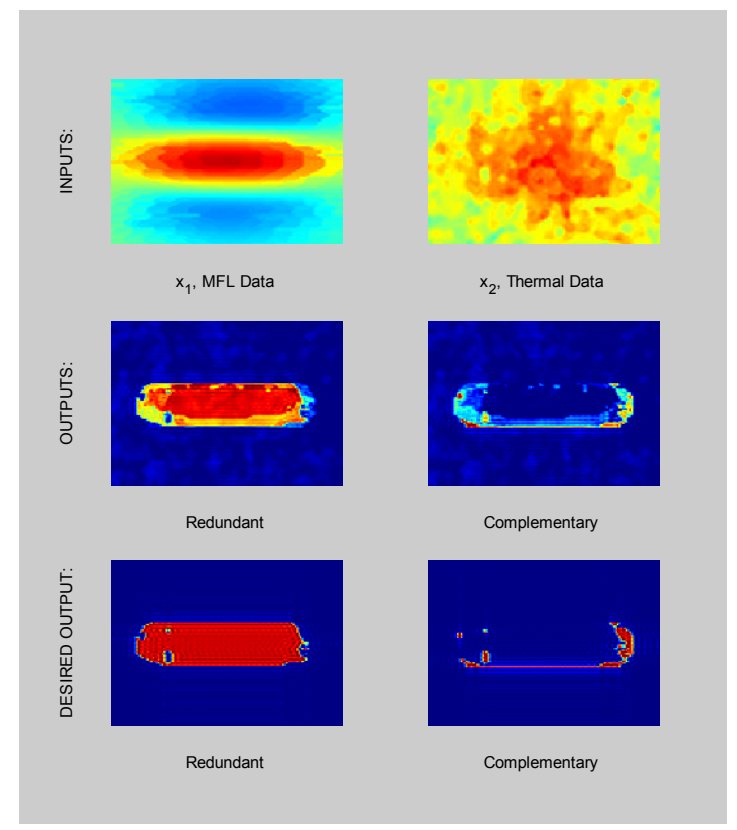

(d) Specimen 01a 
Trial 2: MFL \& Thermal Results (cont.)

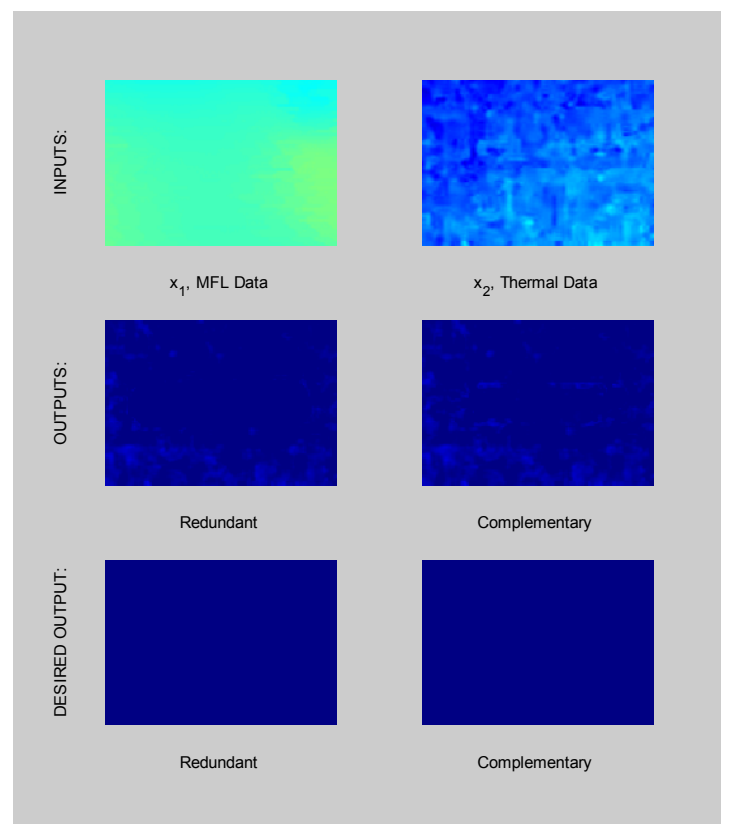

(e) Specimen 10a

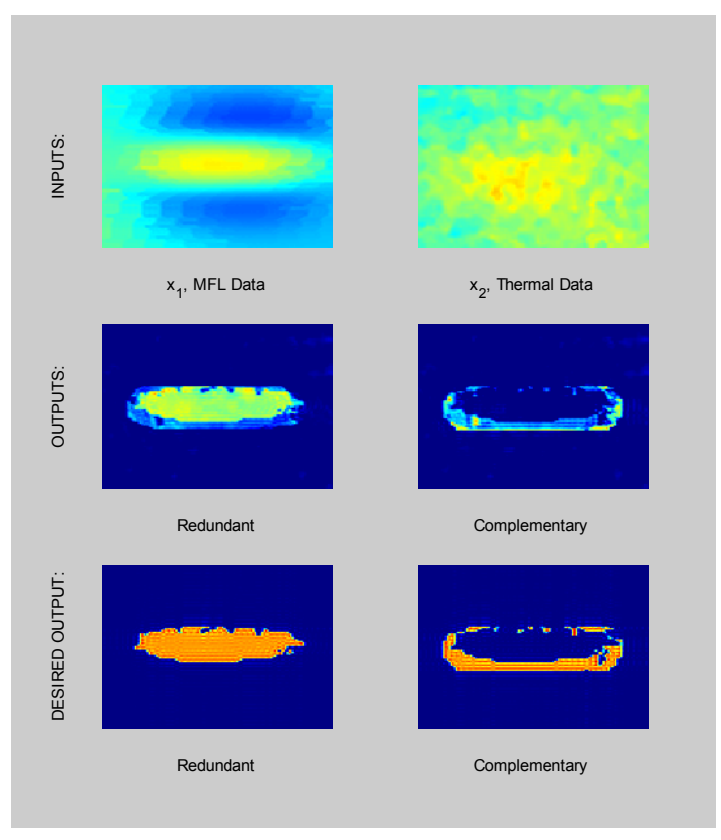

(g) Specimen 12a

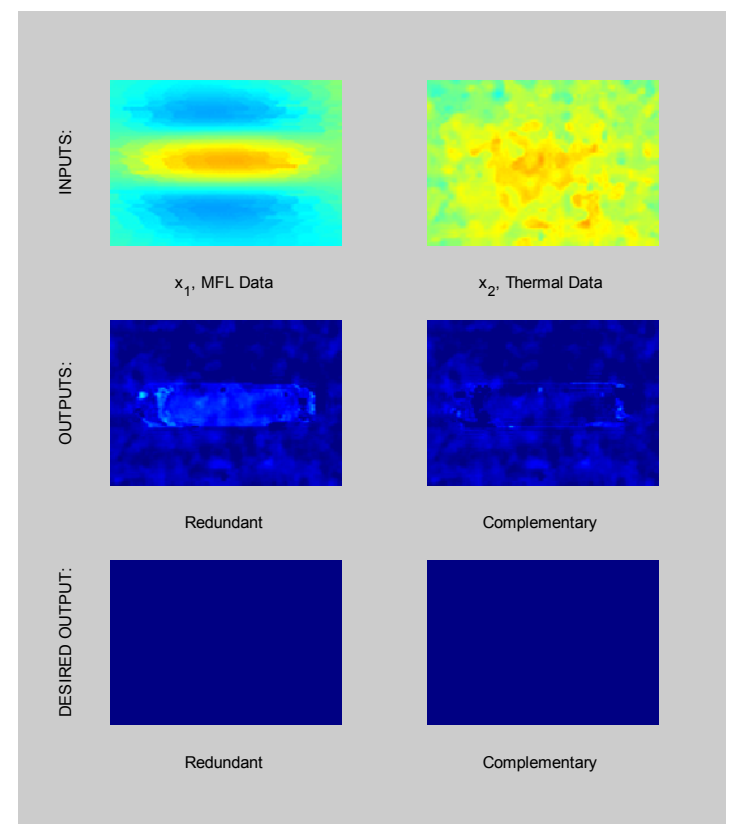

(f) Specimen 13a

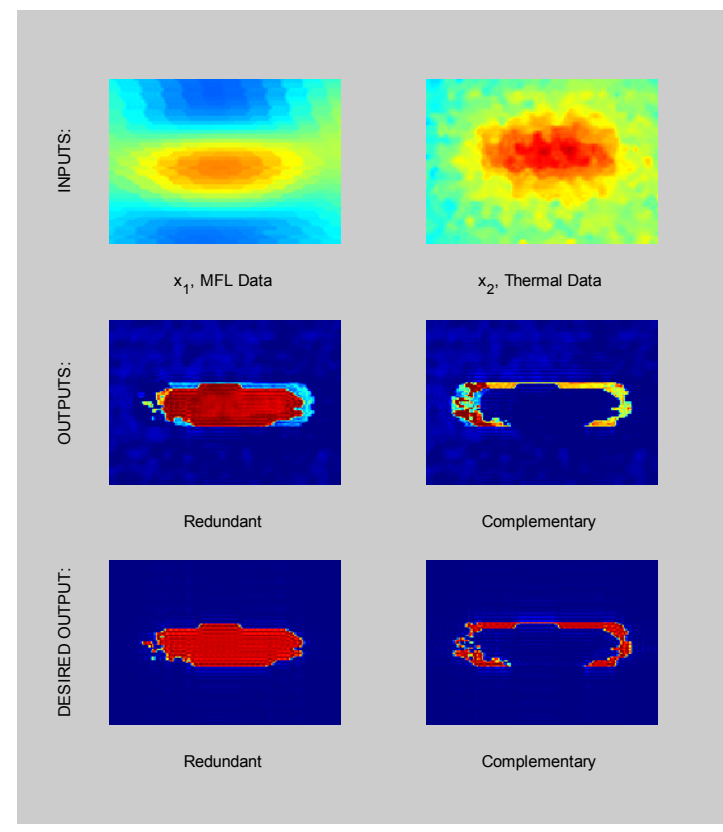

(h) Specimen 11a 
Trial 2: MFL \& Thermal Results (cont.)

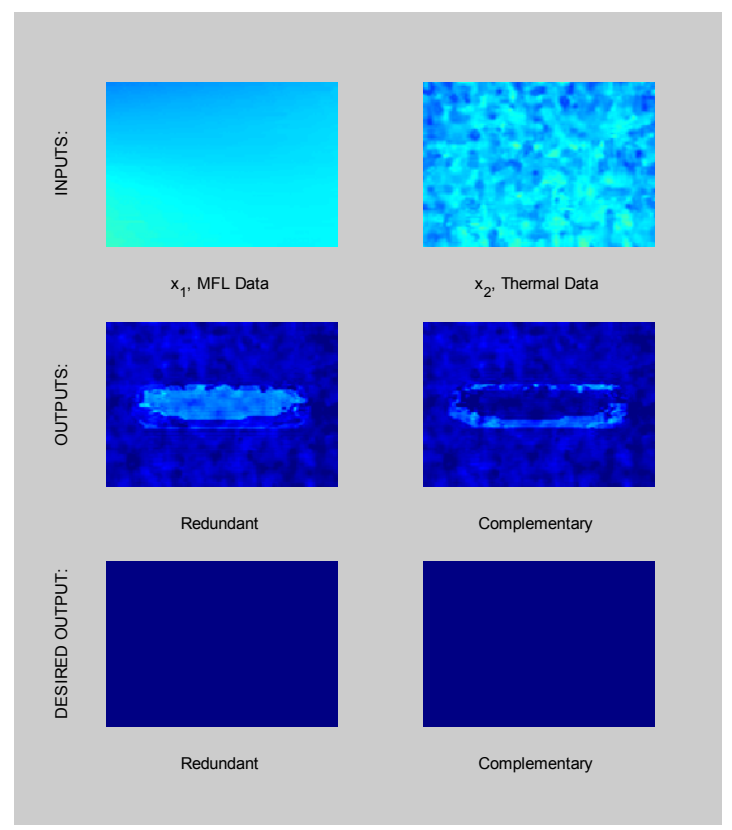

(i) Specimen 20a

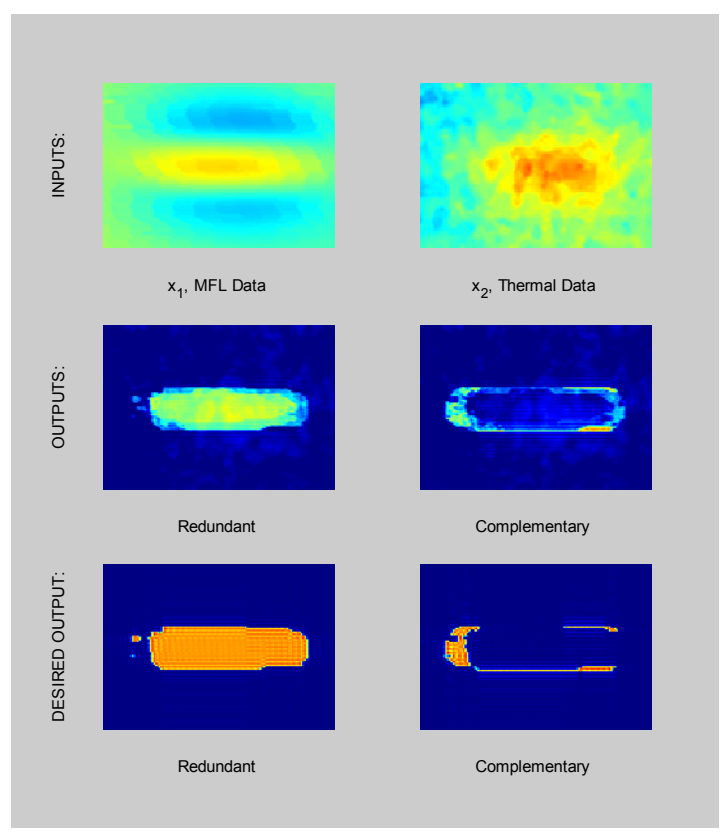

(k) Specimen 22a

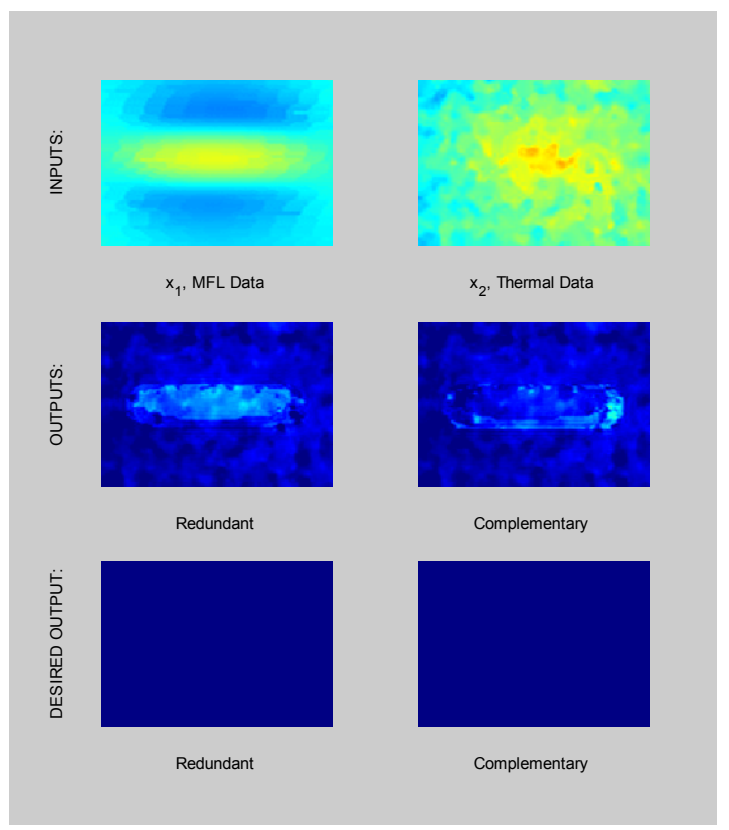

(j) Specimen 23a

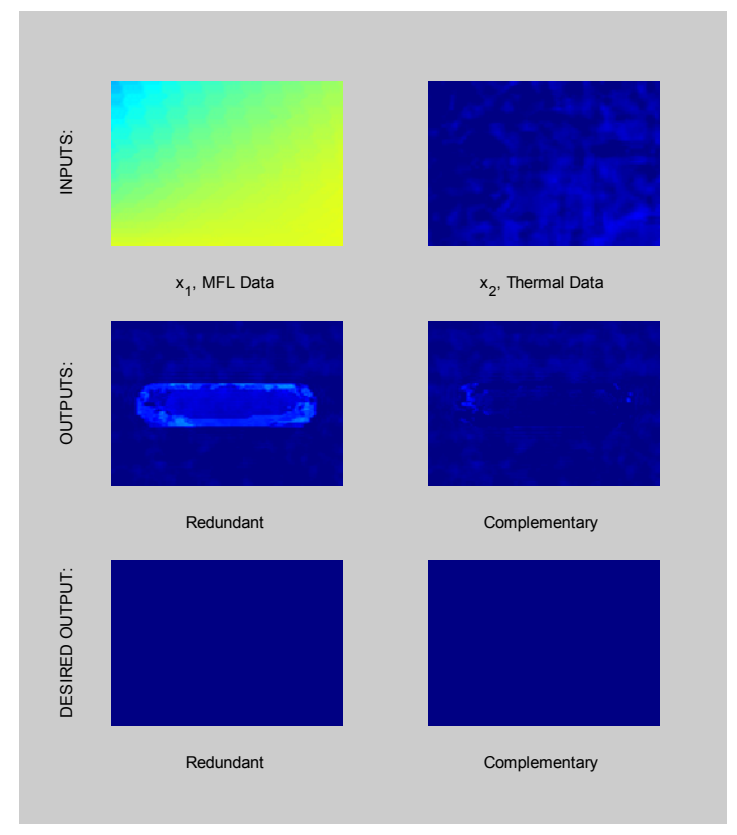

(l) Specimen 00b 
Trial 2: MFL \& Thermal Results (cont.)

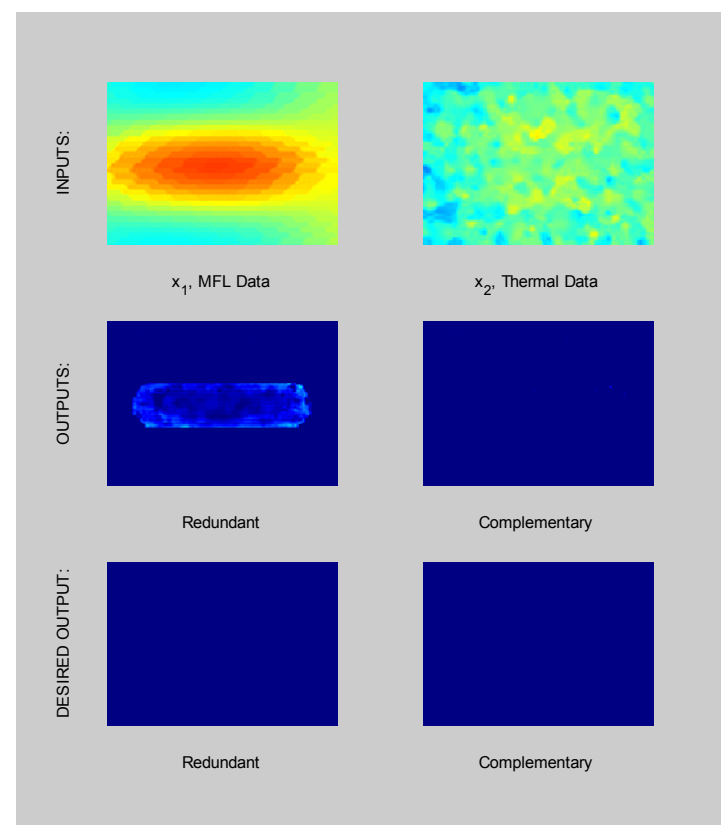

(m) Specimen 03b

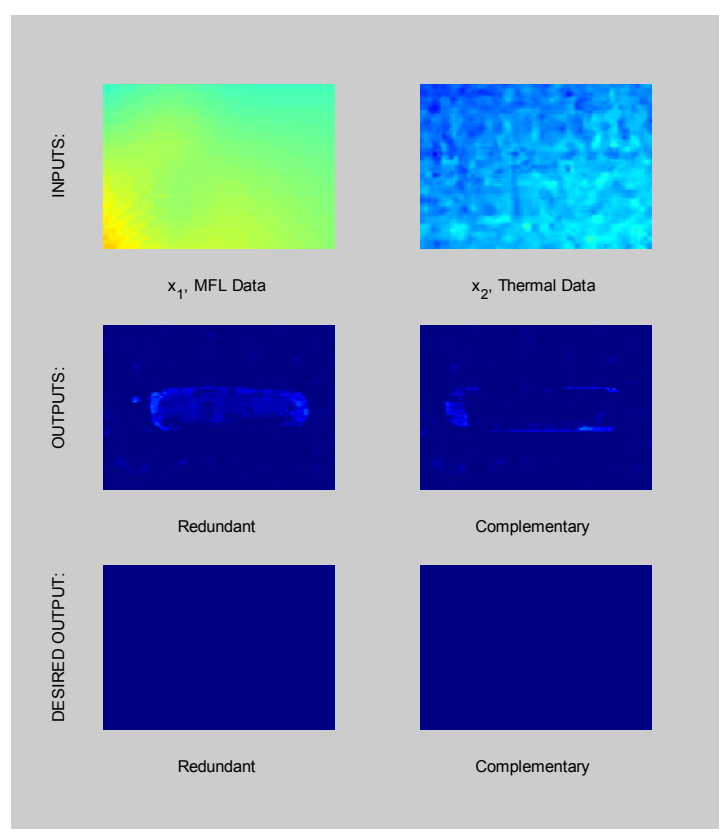

(o) Specimen 10b

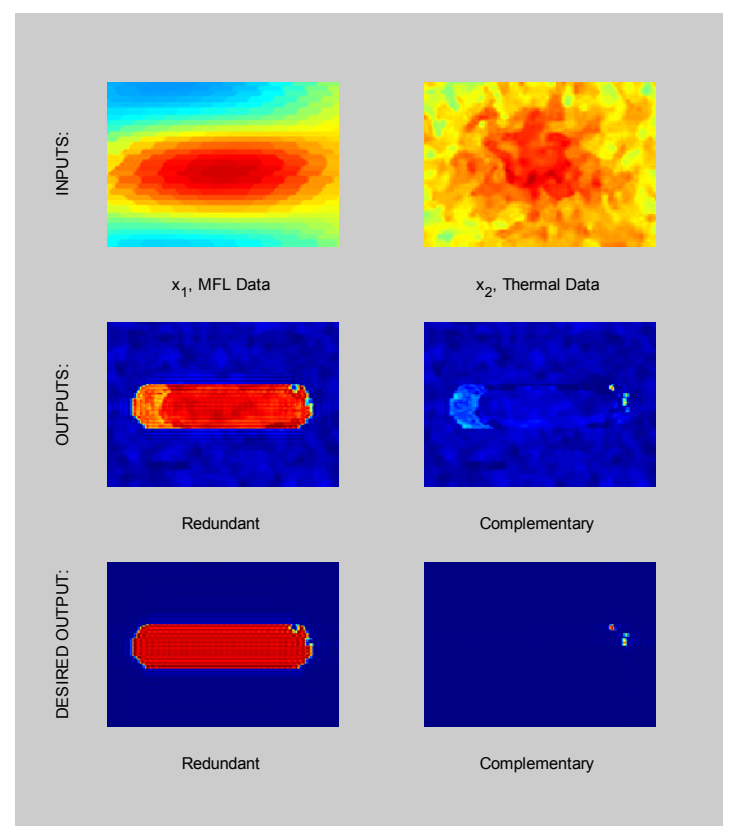

(n) Specimen 01b

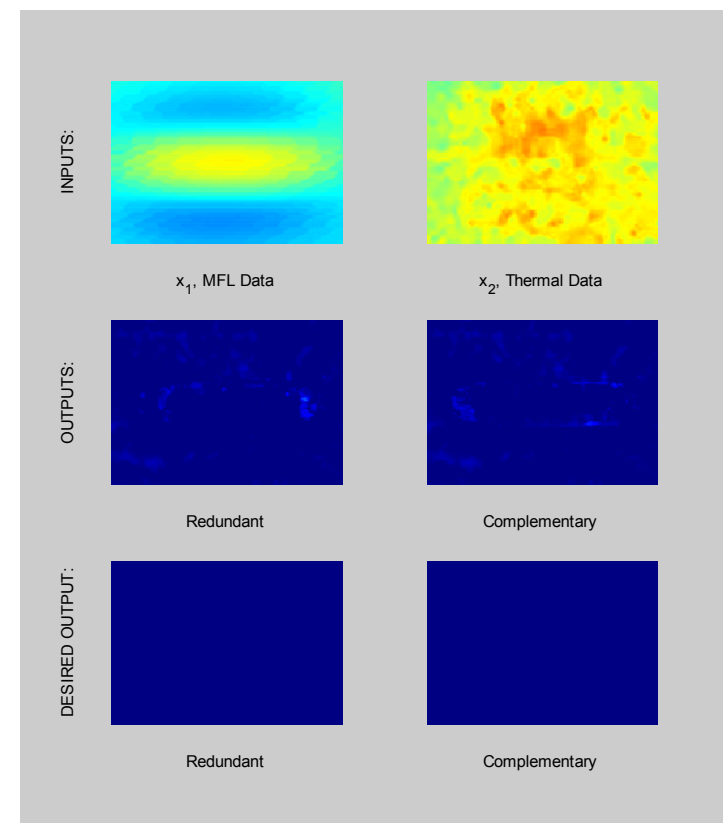

(p) Specimen 13b 
Trial 2: MFL \& Thermal Results (cont.)

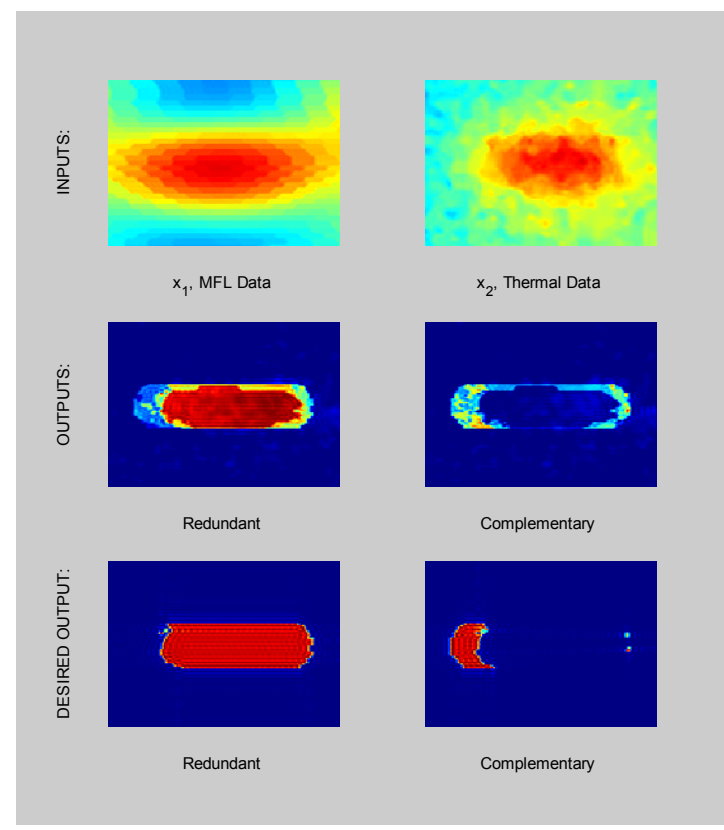

(q) Specimen 11b

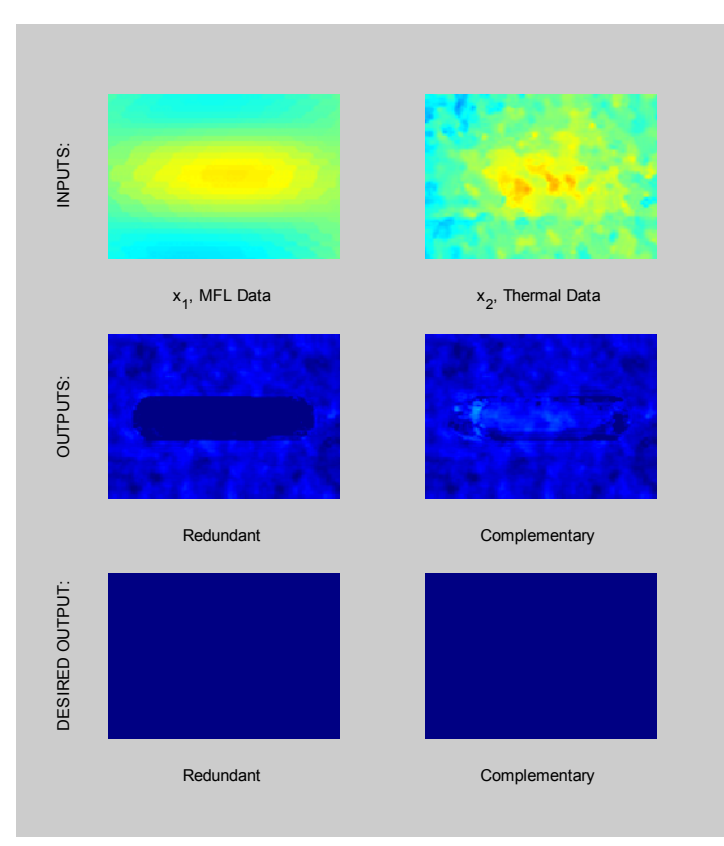

(s) Specimen 23b

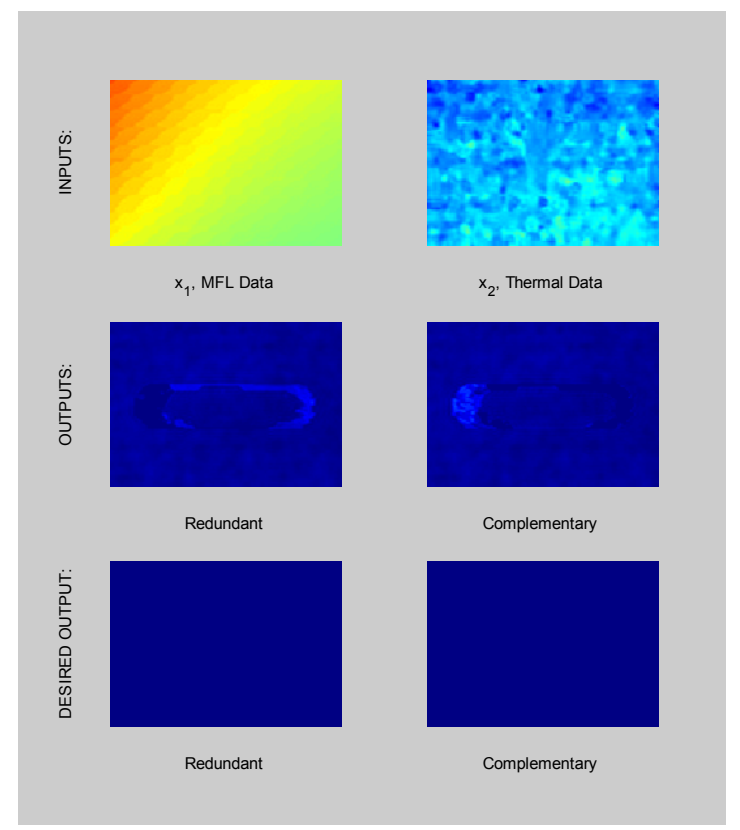

(r) Specimen 20b

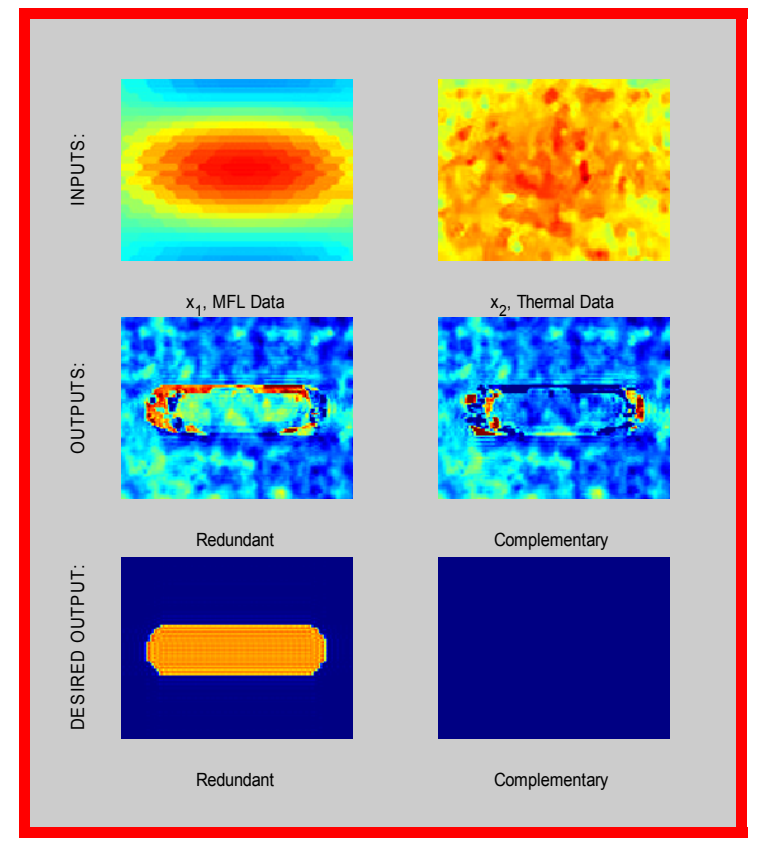

(t) Specimen 02b 
Trial 2: MFL \& Thermal Results (cont.)

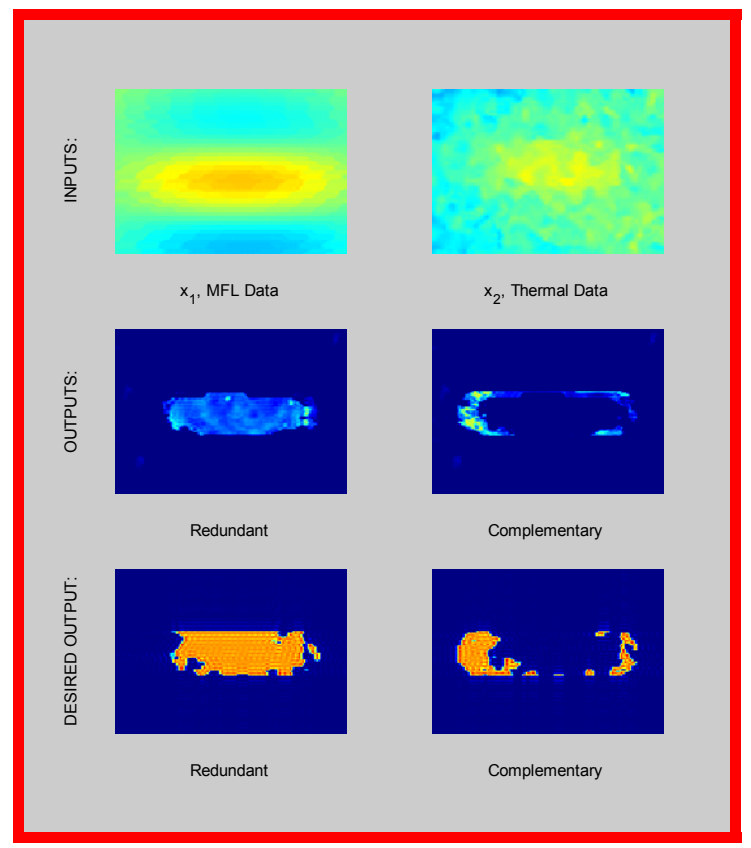

(u) Specimen 12b

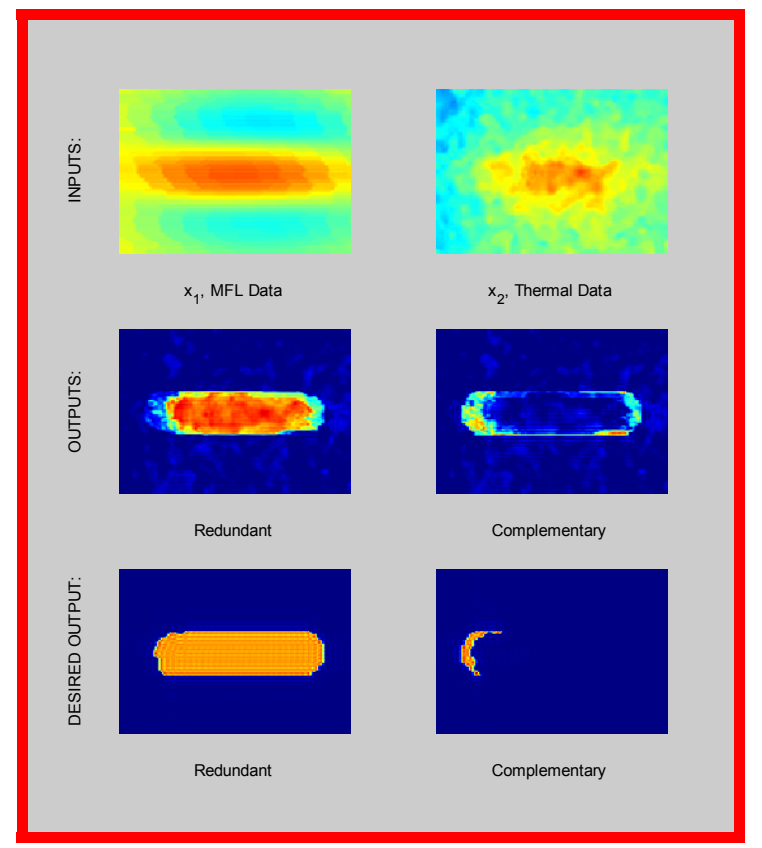

(v) Specimen 22b

Figure 9: MFL \& Thermal combination Trial 2.

Training Data: (a) - (s); Test Data: (t), (u), (v) 
Trial 3: MFL \& Thermal Results

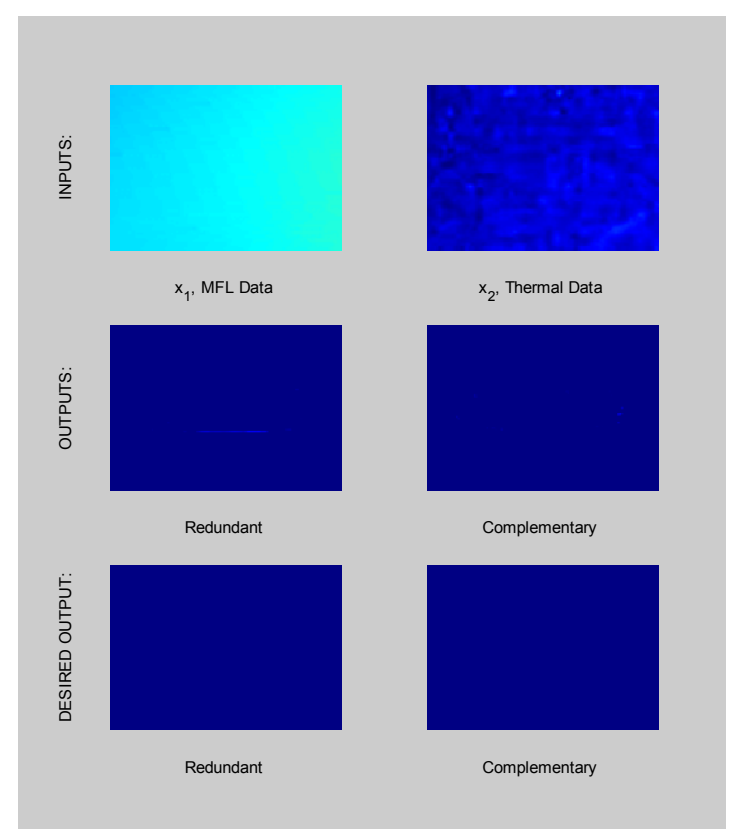

(a) Specimen 00a

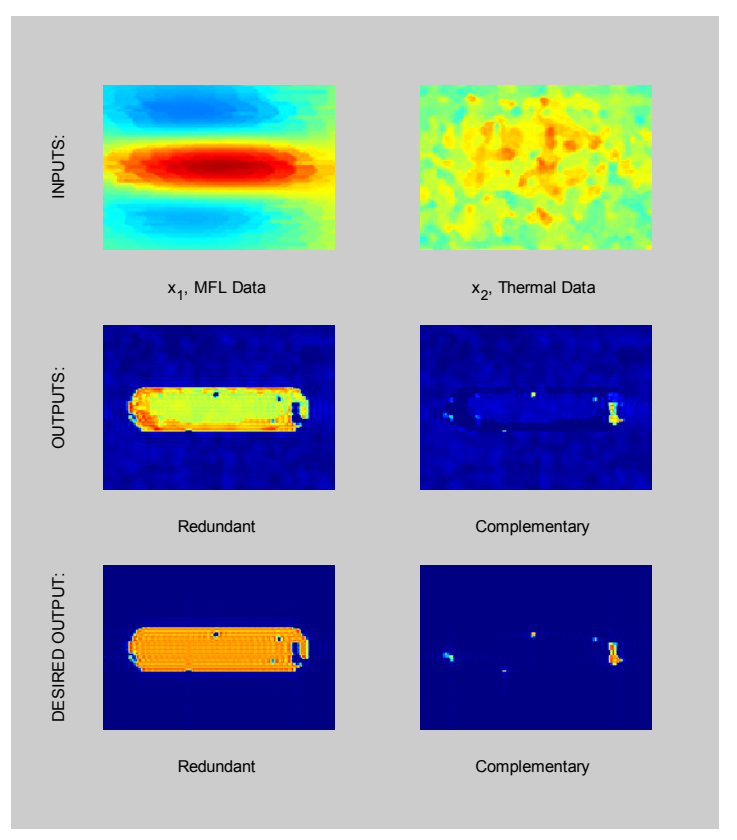

(c) Specimen 02a

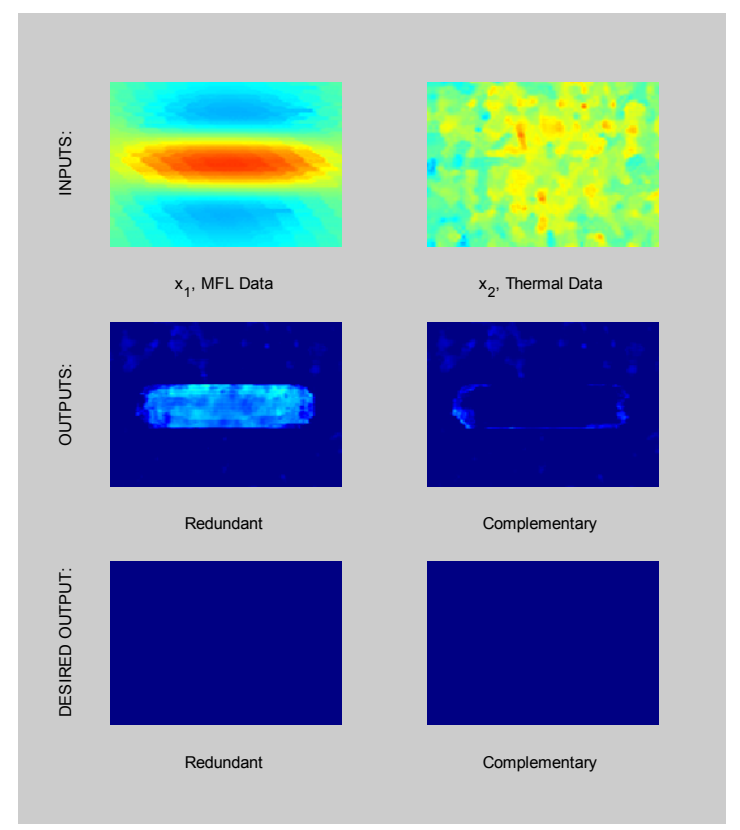

(b) Specimen 03a

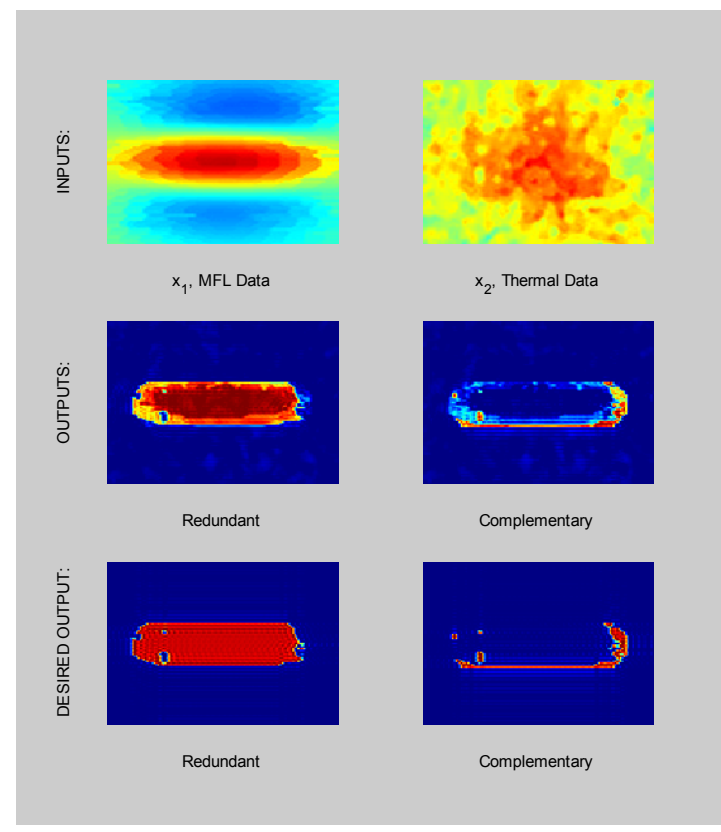

(d) Specimen 01a 
Trial 3: MFL \& Thermal Results (cont.)

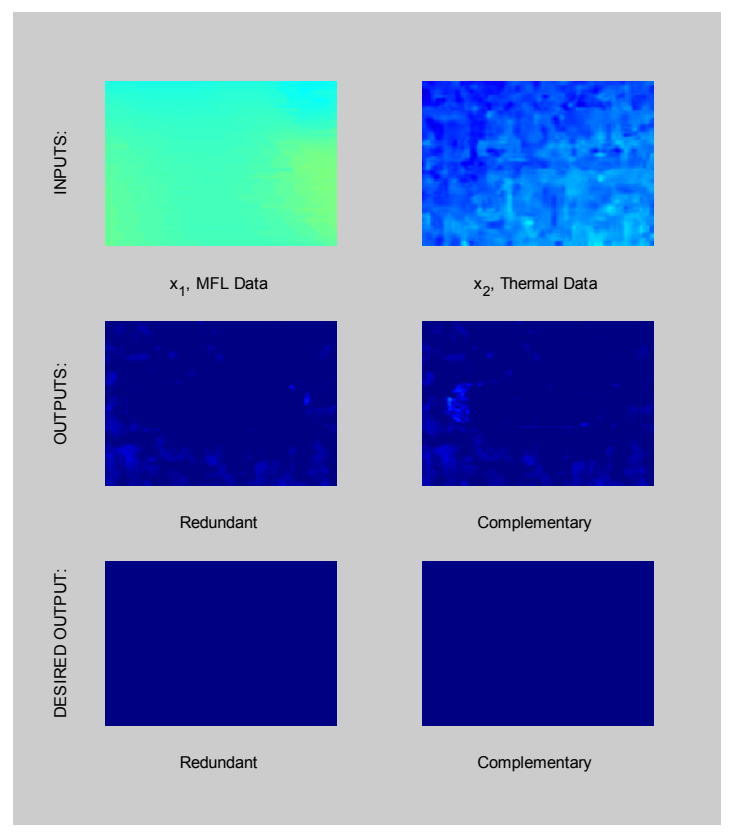

(e) Specimen 10a

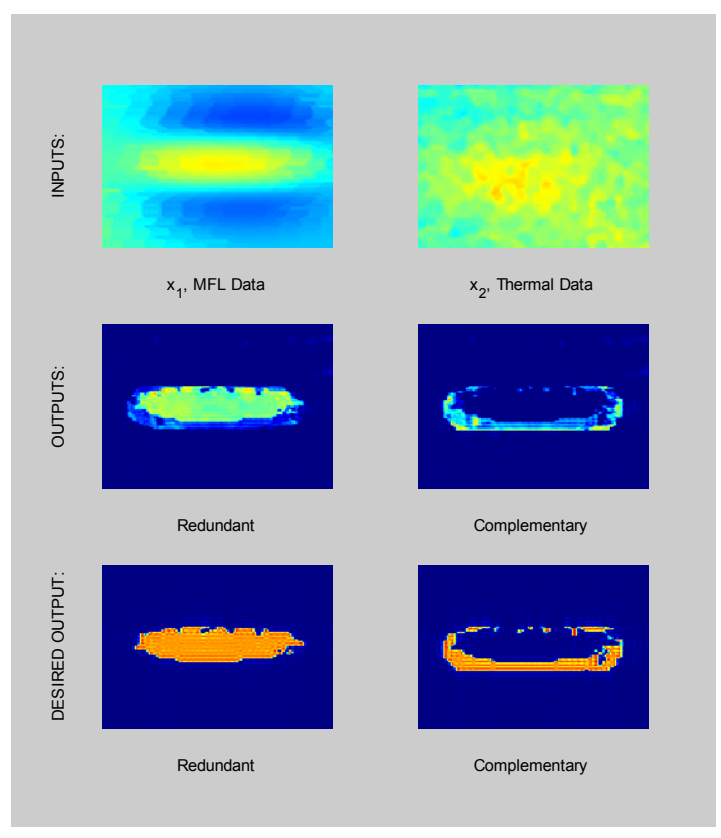

(g) Specimen 12a

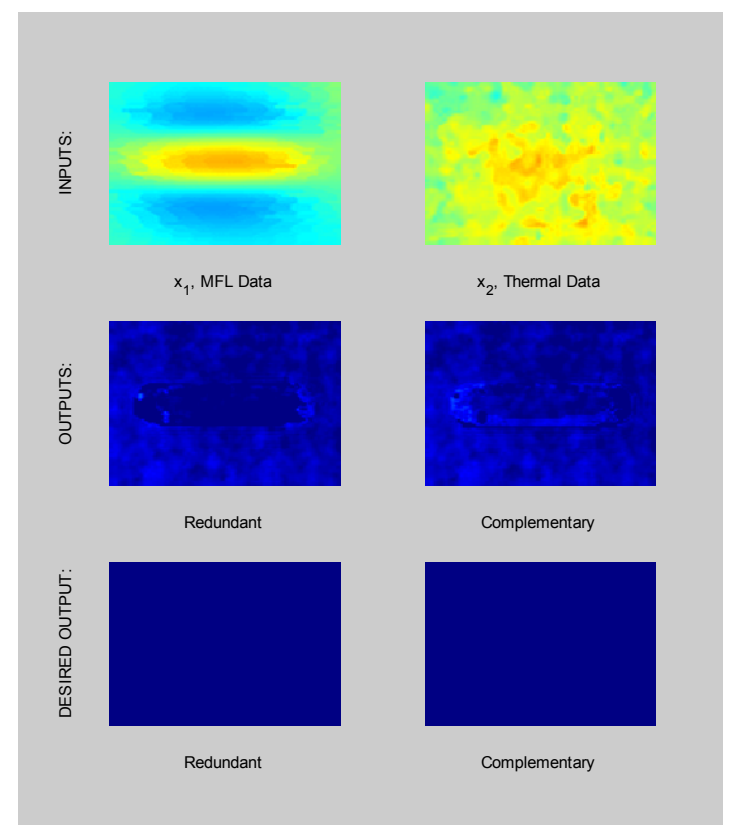

(f) Specimen 13a

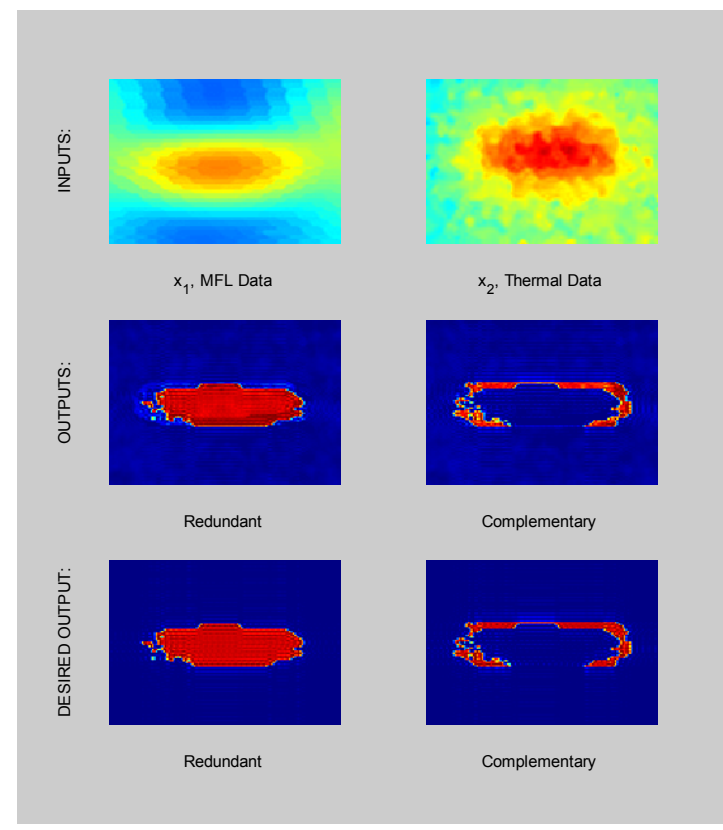

(h) Specimen 11a 
Trial 3: MFL \& Thermal Results (cont.)

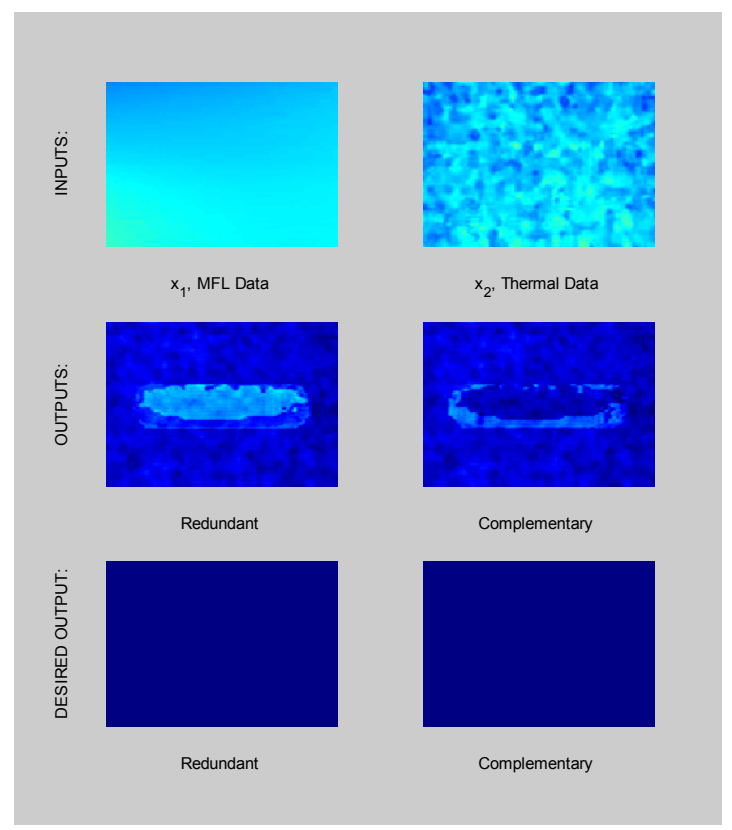

(i) Specimen 20a

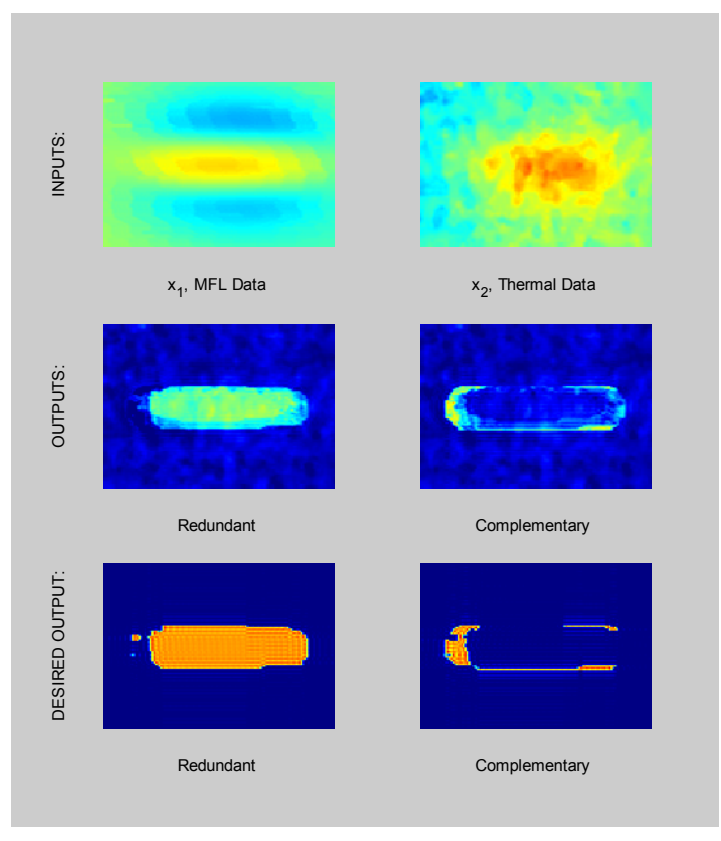

(k) Specimen 22a

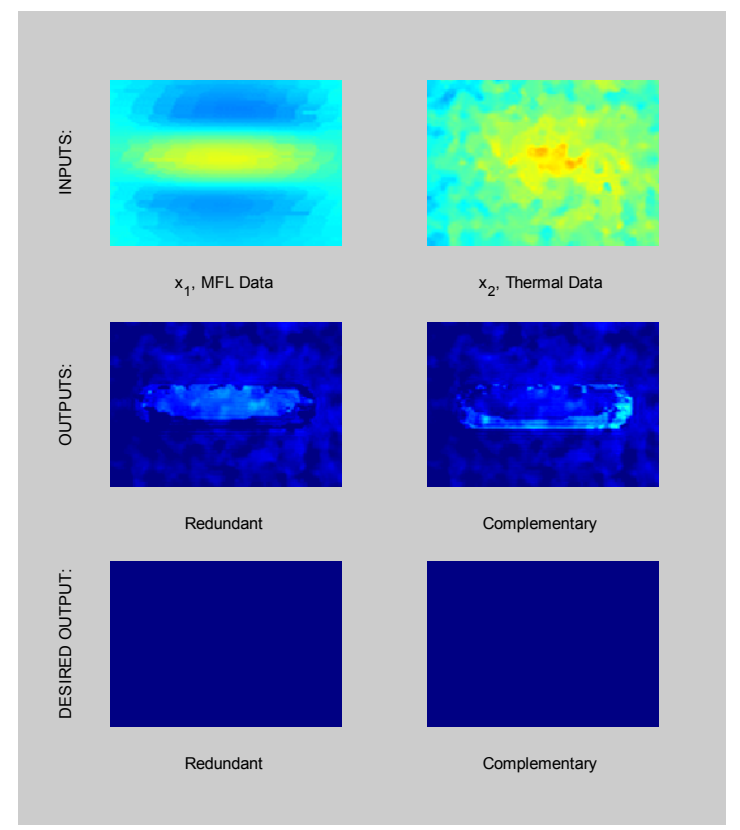

(j) Specimen 23a

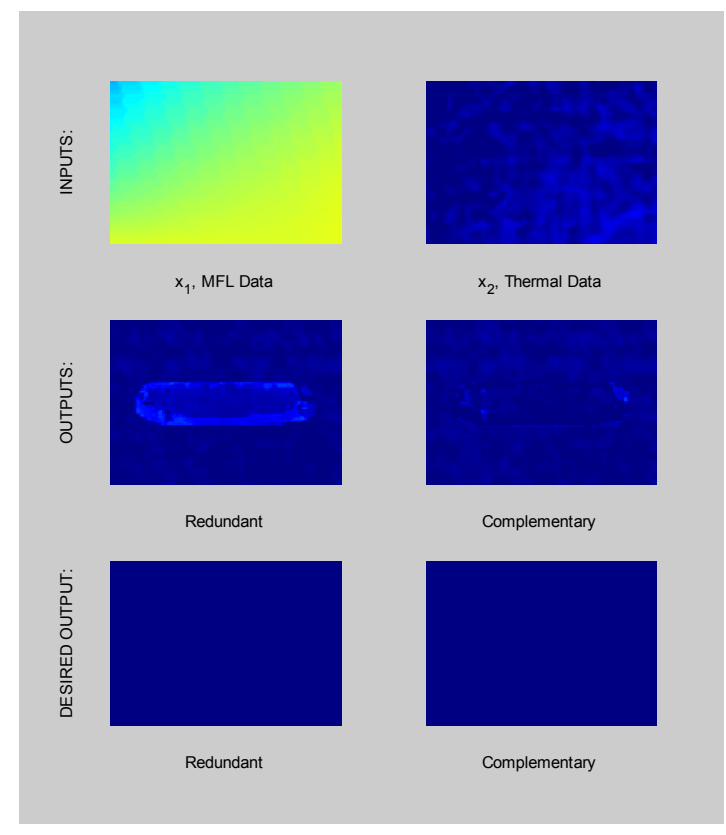

(l) Specimen 00b 
Trial 3: MFL \& Thermal Results (cont.)

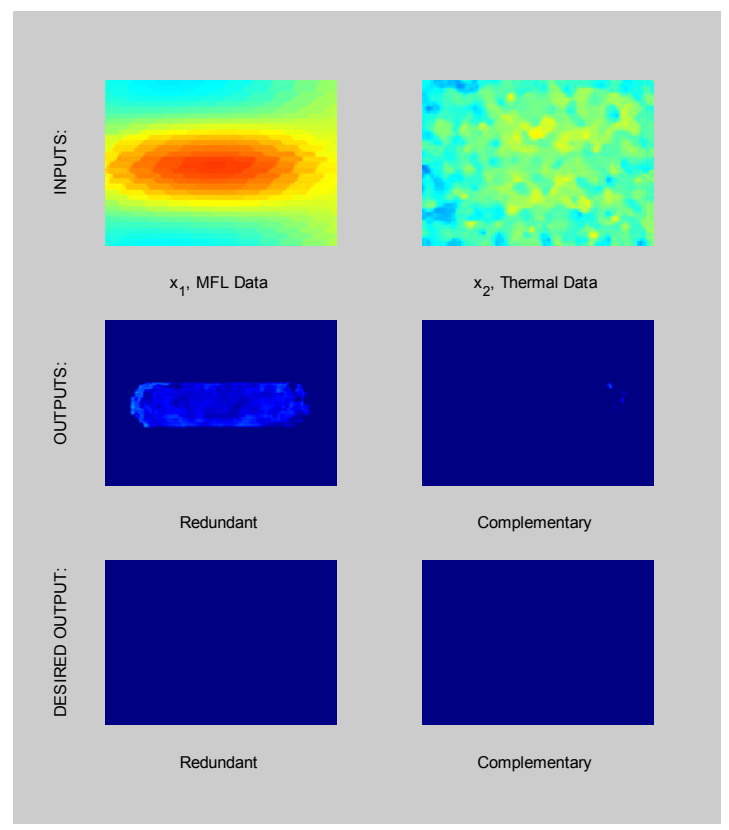

(m) Specimen 03b

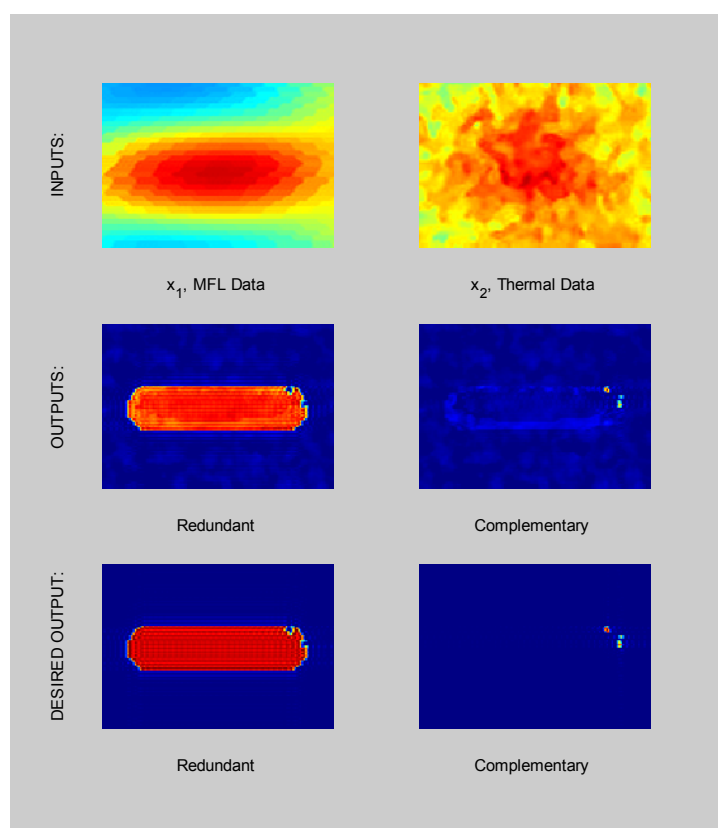

(o) Specimen 01b

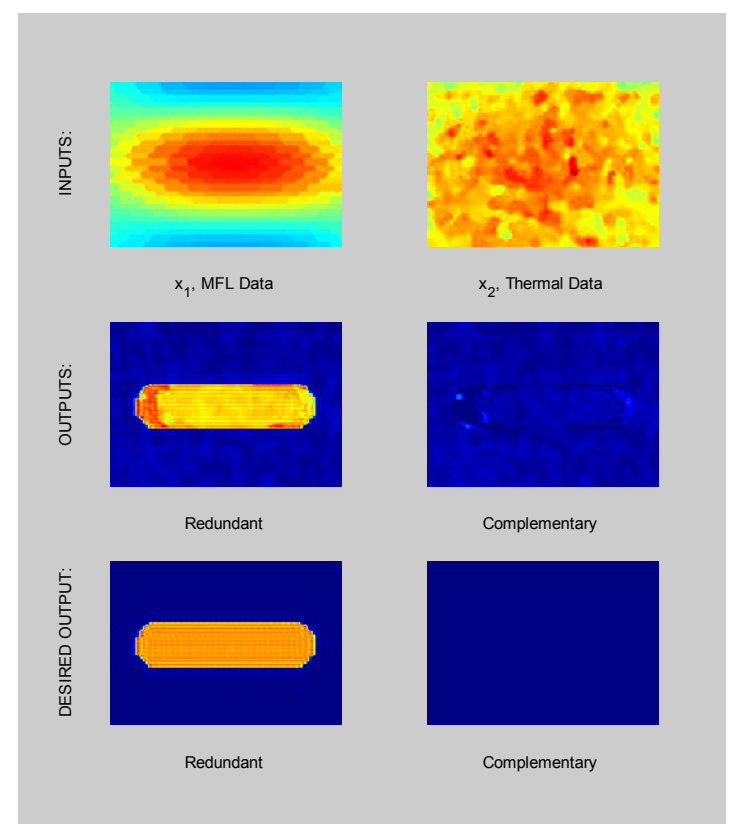

(n) Specimen 02b

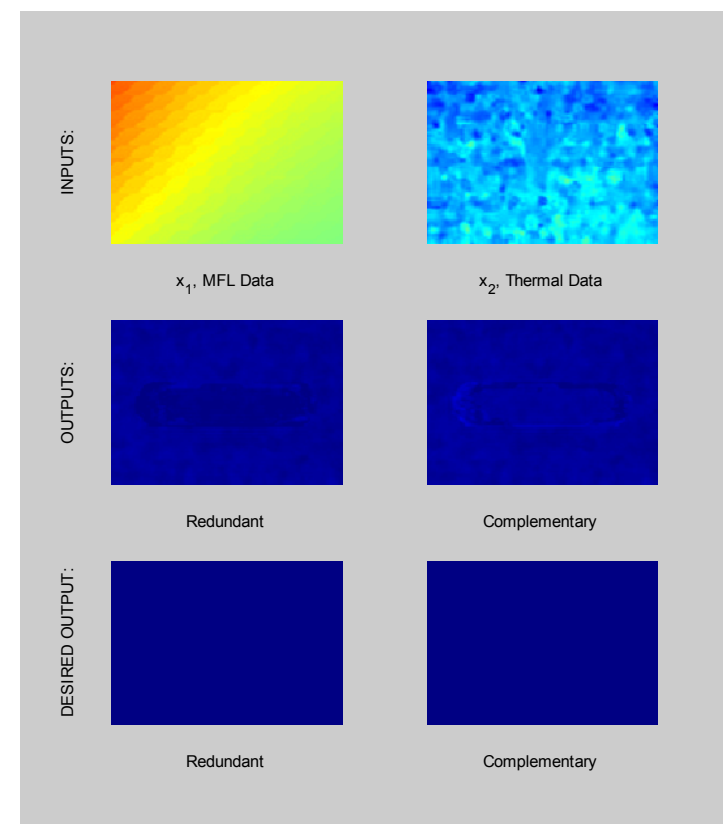

(p) Specimen 20b 
Trial 3: MFL \& Thermal Results (cont.)

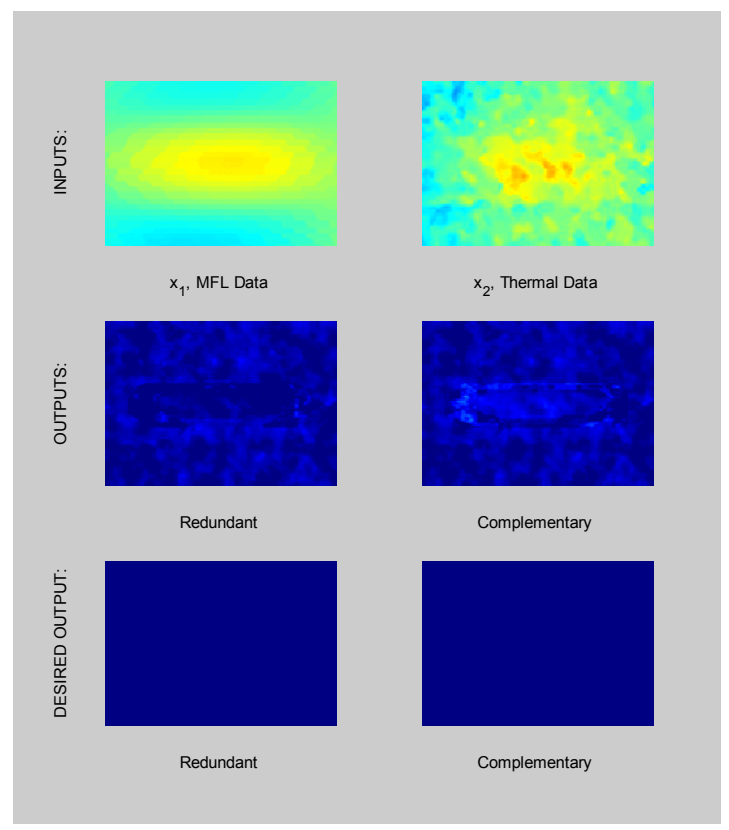

(q) Specimen 23b

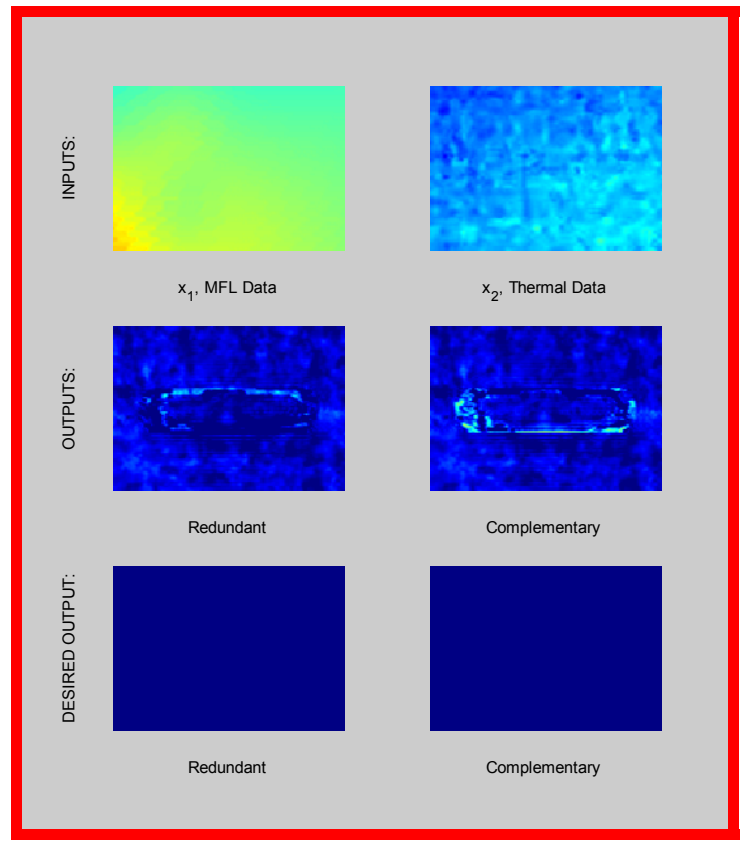

(s) Specimen 10b

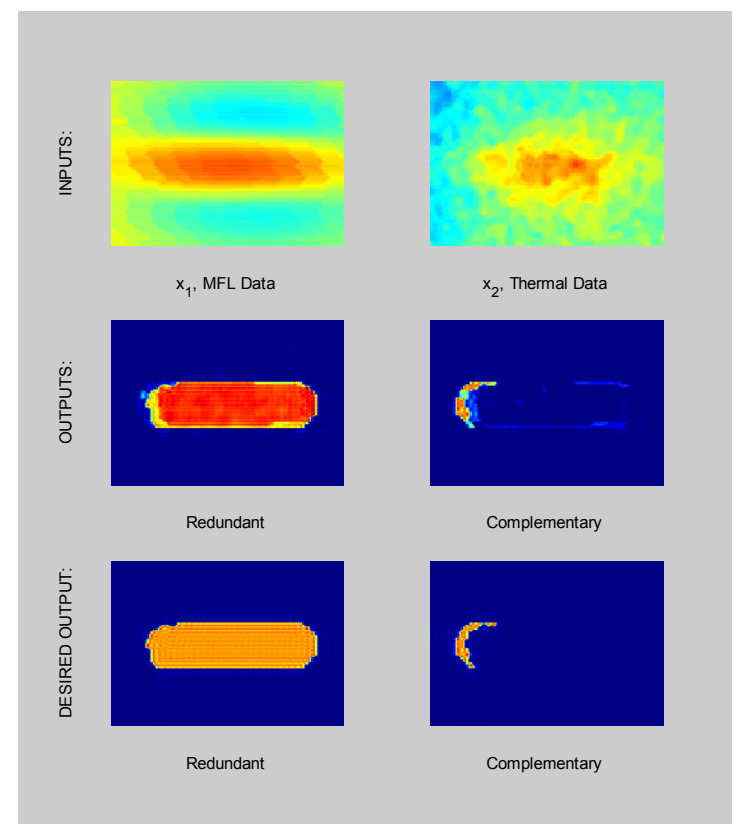

(r) Specimen 22b

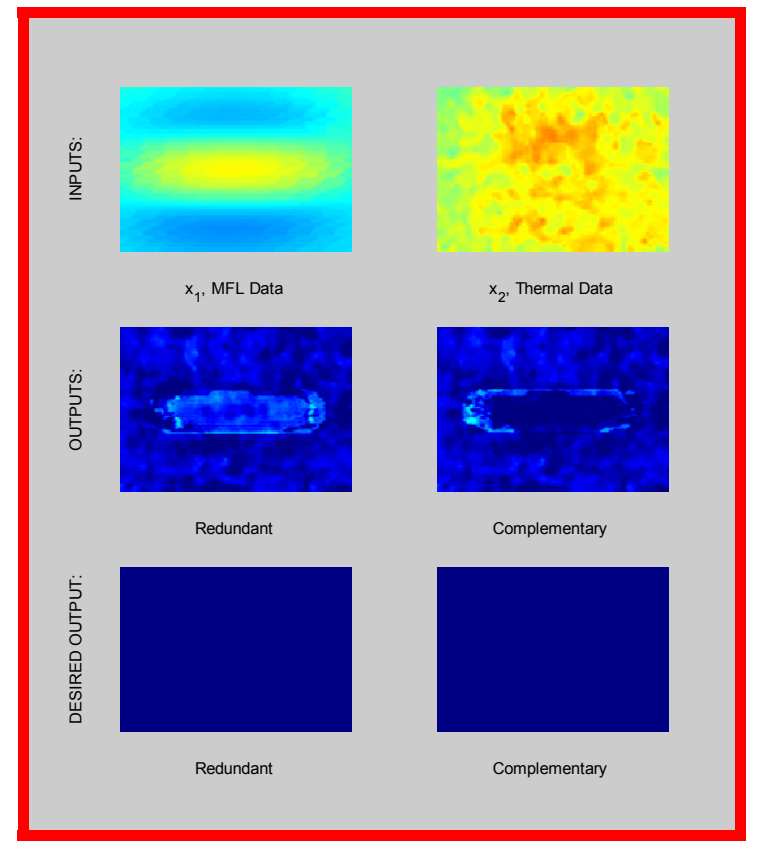

(t) Specimen 13b 
Trial 3: MFL \& Thermal Results (cont.)

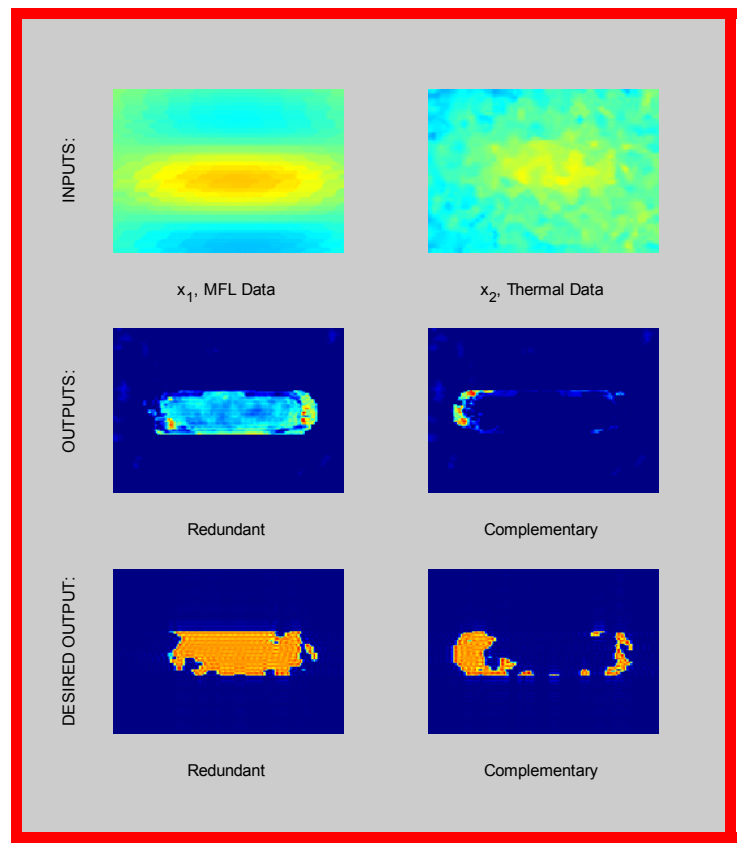

(u) Specimen 12b

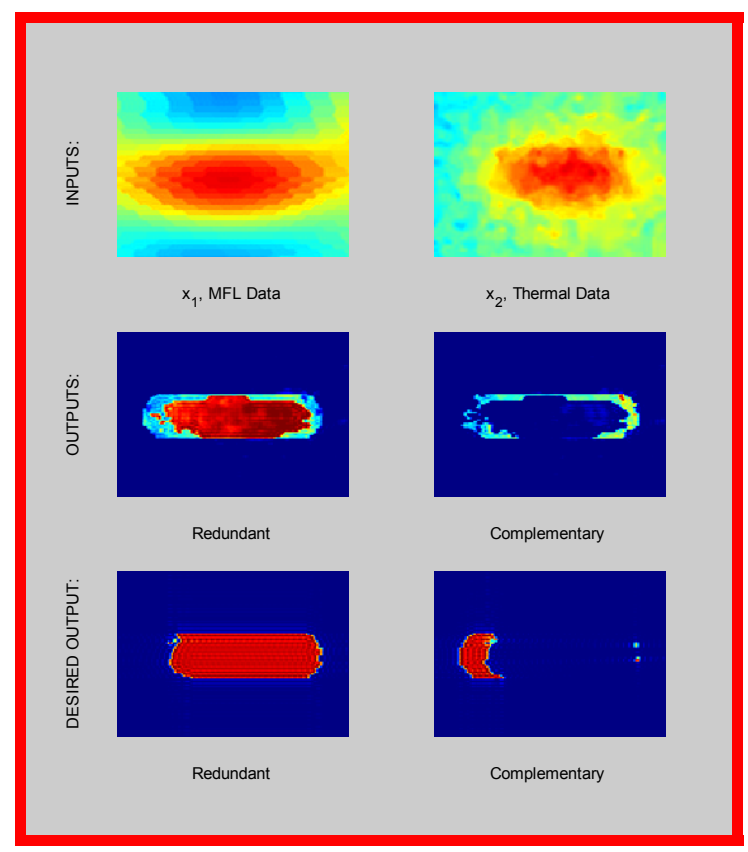

(v) Specimen 11b

Figure 10: MFL \& Thermal combination Trial 3.

Training Data: (a) - (r); Test Data: (s), (t), (u), (v) 
In order to perform a redundant and complementary data extraction process on acoustic emission data it is first necessary to define how the location of $\mathrm{AE}$ source points corresponds to the position and shape of the actual defect. Unlike the homogeneous NDE sources, acoustic emission data does not provide the user with geometrical shape information of the defect, but only provides an estimation of where the defect is located. To perform redundant and complementary data extraction it is first necessary to relate the acoustic emission point data to a specific defect location area. Once this process has been performed the homogeneous data fusion process can be employed to extract the redundant and complementary information.

The acoustic emission testing performed in the laboratory seemed to indicate that the acoustic emission source locations do not fall directly over the defect area, but rather form clusters located around the defect. From this information the assumption can be made that the defect is located somewhere in the area surrounded by the AE source locations. In order to perform the data fusion process it is necessary to characterize this area of the defect so it can be combined with the other NDE testing modalities. The first method in characterizing this area was to perform a K-means clustering algorithm on the raw AE data to determine how many clusters the data had been formed into and which points fell into each cluster. Figure 11 shows the AE data from the uniaxial specimen with the 0.16 inch deep defect after the cluster algorithm has been performed. In this case two separate classes of data have been clearly defined on either side of the defect.

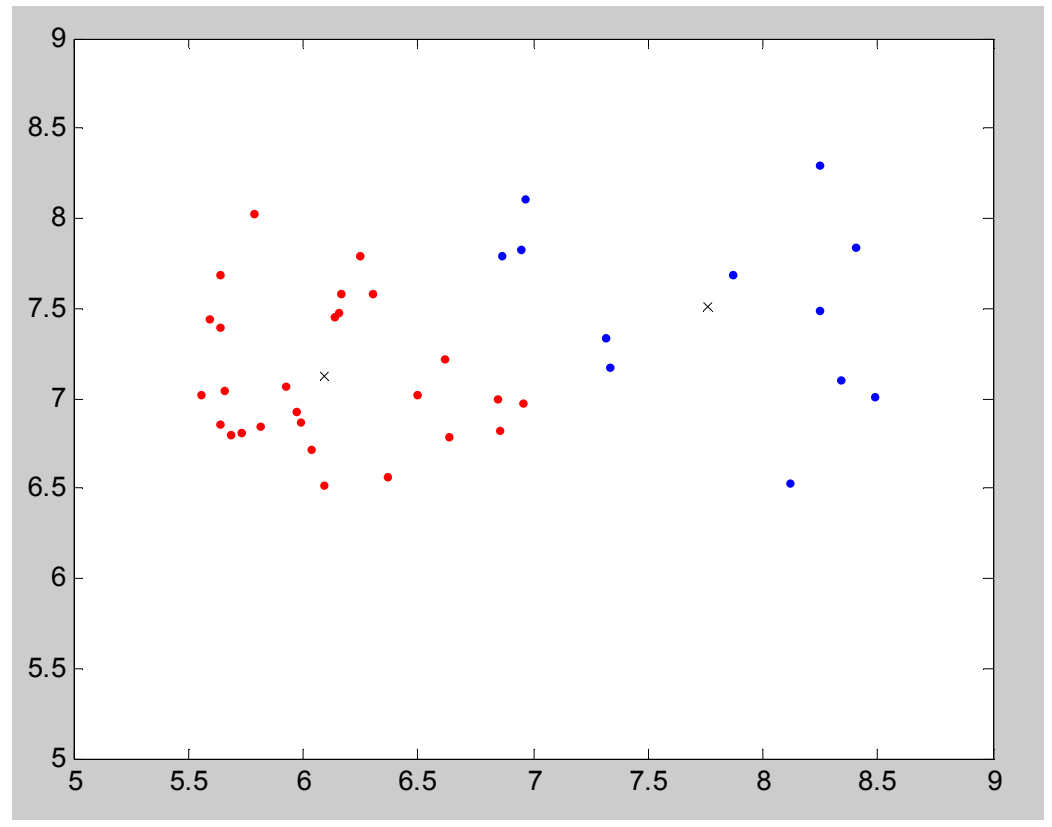

Figure 11: K-means clustering on AE data of uniaxial specimen with 0.16 " deep defect. 
After the two classes of data have been defined, a Parzen windows density estimation [2] is performed on each class separately which can be seen in Figure 12. The Parzen windows approximation fits a two-dimensional Gaussian plot over each class of the data to demonstrate the areas of higher density of the AE sources.

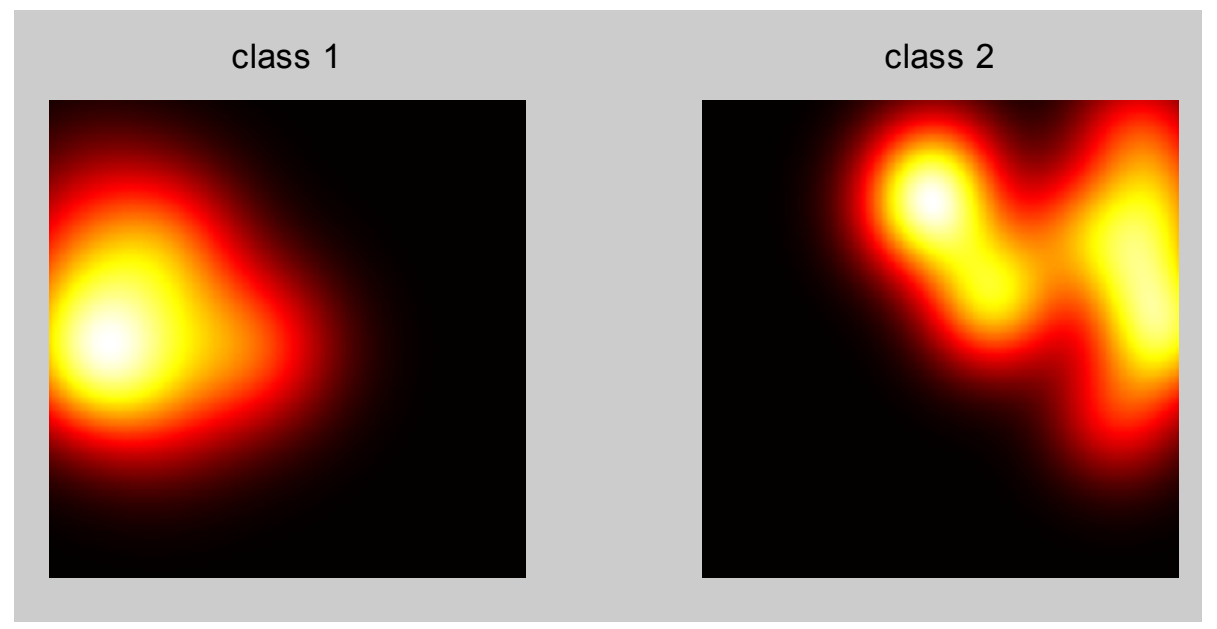

Figure 12: Parzen windows density estimation of each class separately.

The Parzen window images are then overlaid on top of each other. Where the density estimations overlap becomes the region in which the $\mathrm{AE}$ data predicts the presence of a defect. To perform this process the Parzen window images are converted to binary images seen in Figure 13. A logical AND is then performed between the two image matrices to determine where the images overlap.

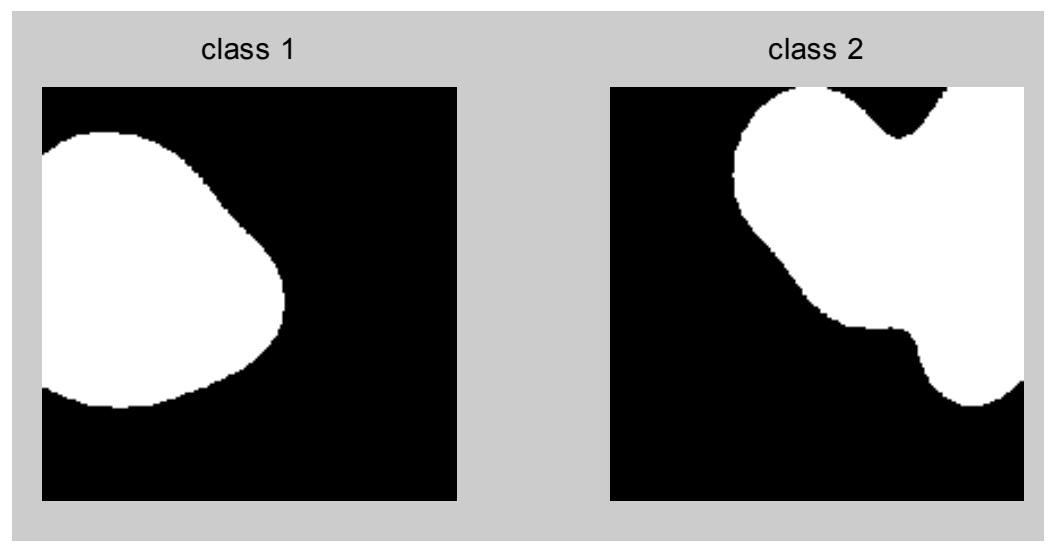

Figure 13: Binary representation of Parzen windows AE images.

For the AE tests performed in this project, the number of clusters of data surrounding the defect could vary from one to four classes. The overlapping areas of the Parzen windows images were then weighted from 0 to 1 in increments of 0.25 , i.e., if two classes overlap, the overlapped area will be weighted at 50 percent, and for three classes overlapping 75 percent and so on. This allows for a high volume of data surrounding the defect to have a stronger influence on the data 
fusion process. Figure 14 shows the resulting defect area represented from the acoustic emission source data shown above in Figure 11.

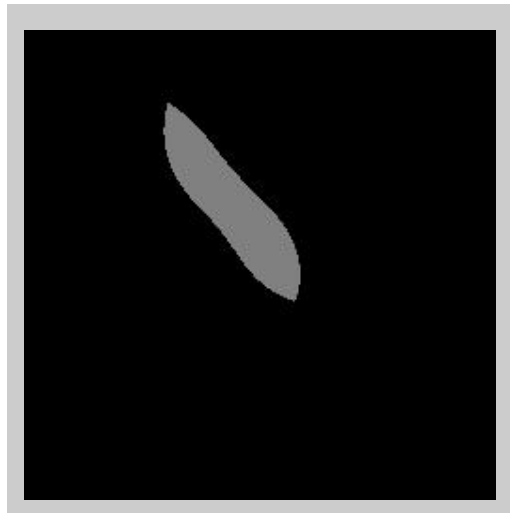

Figure 14: Defect location representation from AE data.

The above process was performed on all of the acoustic emission data to generate AE location estimation plots. The resulting images seen in Figure 15 can then be input to the redundant and complementary data extraction algorithm.

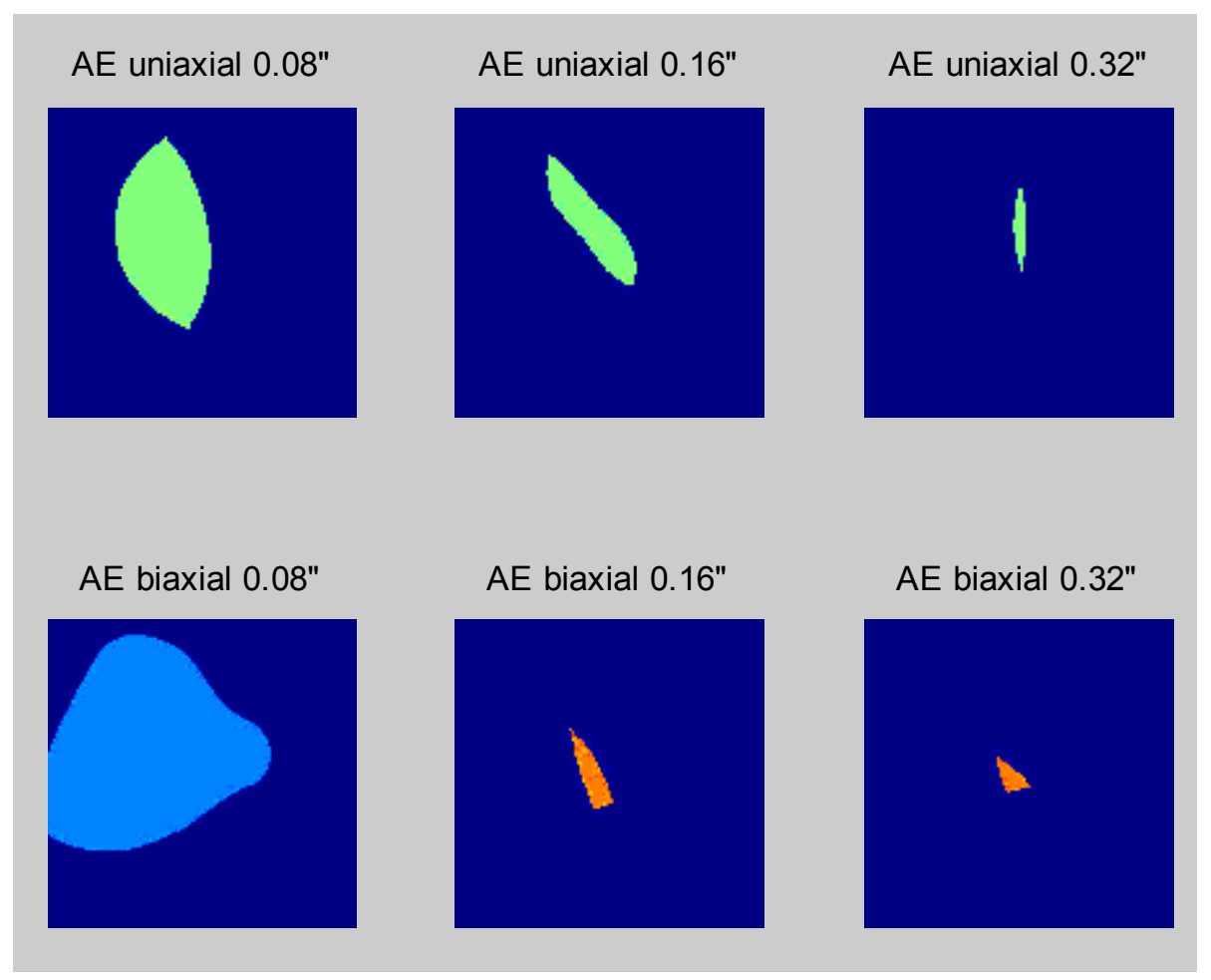

Figure 15: AE location estimation plots for the data fusion algorithm. 
The heterogeneous redundant and complementary data fusion combination process was performed on ultrasonic and acoustic emission data taken of test specimen suite 2. Since only six data instances were available for training and testing purposes only two trials were performed. Trials 1 and 2 seen in Table 4 and 5 show the division of training and test data.

Table 4: Trial 1 training and test dataset.

\begin{tabular}{|c|c|c|c|c|}
\hline Specimen \# & Type & Plate Thickness (in) & Indication & Crack Depth (in) \\
\hline Uni08 & Uniaxial & 0.5 & SCC & 0.08 \\
\hline Uni16 & Uniaxial & 0.5 & SCC & 0.16 \\
\hline Uni32 & Uniaxial & 0.5 & SCC & 0.32 \\
\hline Bi08 & Biaxial & 0.5 & SCC & 0.08 \\
\hline Bi16 & Biaxial & 0.5 & SCC & 0.16 \\
\hline Bi32 & Biaxial & 0.5 & SCC & 0.32 \\
\hline
\end{tabular}

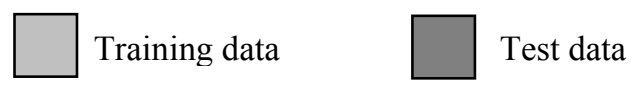

Table 5: Trial 2 training and test dataset.

\begin{tabular}{|c|c|c|c|c|}
\hline Uni08 & Uniaxial & 0.5 & SCC & 0.08 \\
\hline Uni16 & Uniaxial & 0.5 & SCC & 0.16 \\
\hline Uni32 & Uniaxial & 0.5 & SCC & 0.32 \\
\hline Bi08 & Biaxial & 0.5 & SCC & 0.08 \\
\hline Bi16 & Biaxial & 0.5 & SCC & 0.16 \\
\hline Bi32 & Biaxial & 0.5 & SCC & 0.32 \\
\hline
\end{tabular}

Training data $\quad \square$ Test data 
Acoustic Emission \& Ultrasound Data Fusion Results

Trial 1: AE \& UT Results

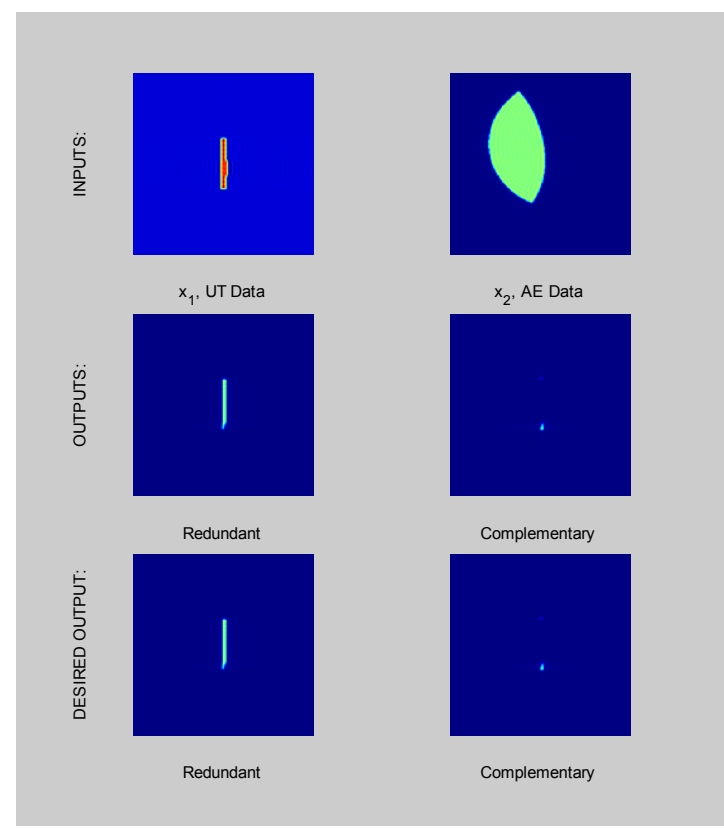

(a)

Trial 1: AE \& UT Results (cont.)

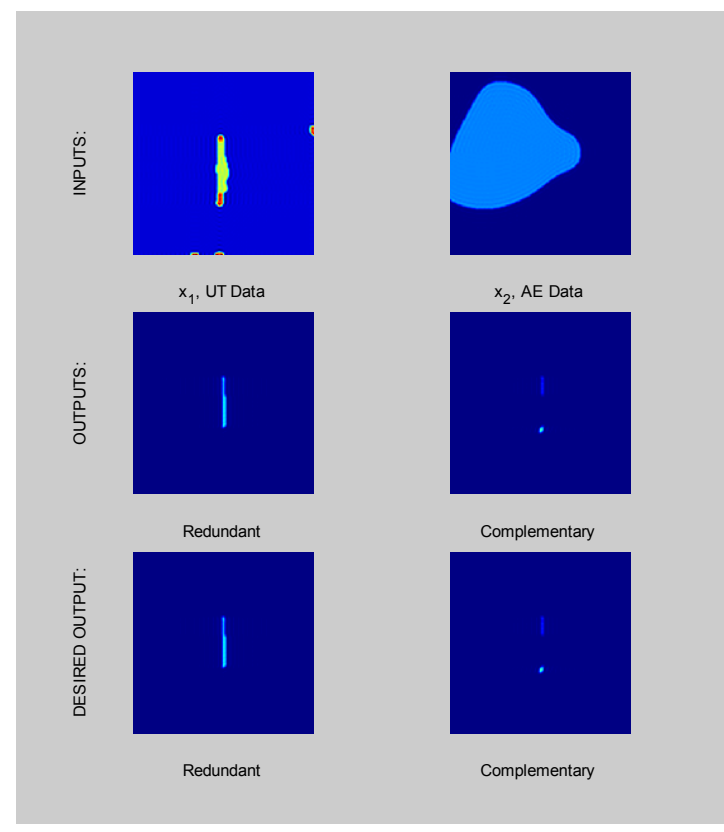

(c)

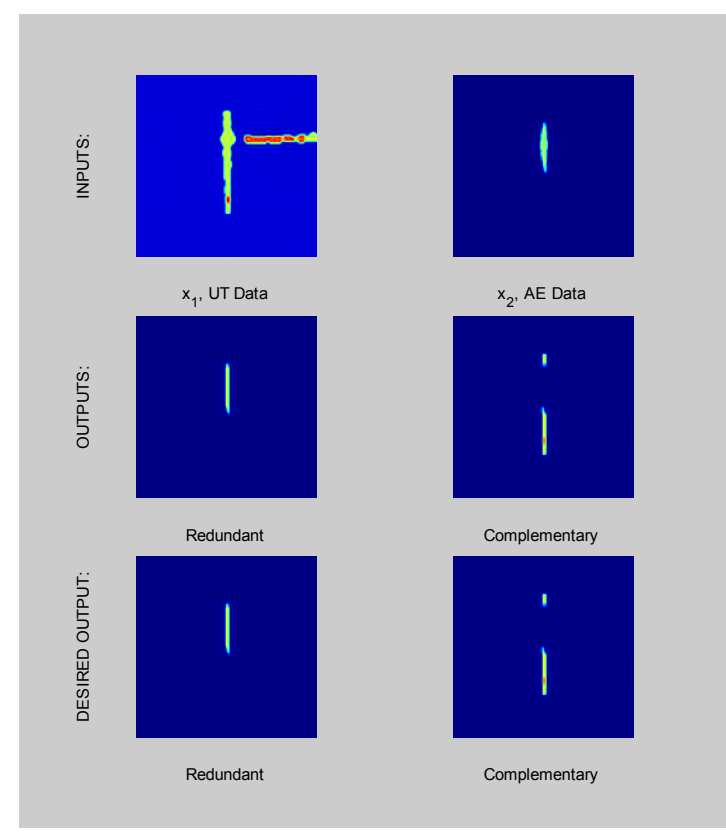

(b)

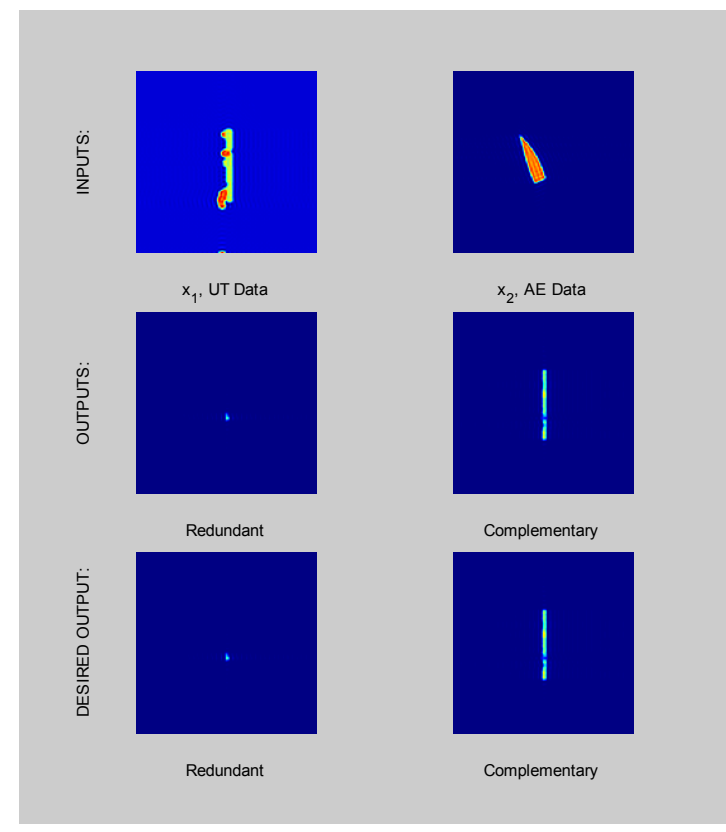

(d) 


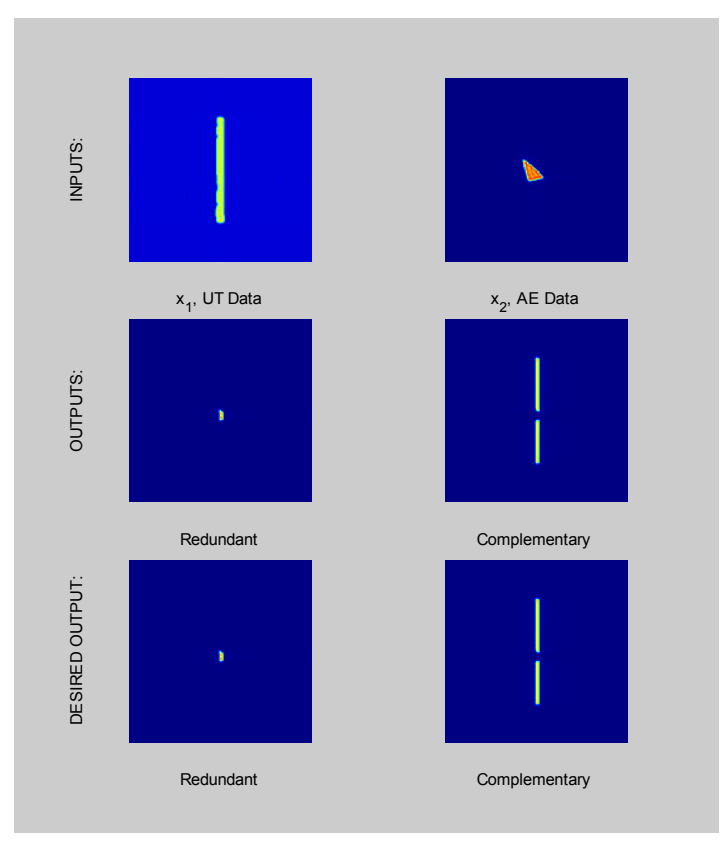

(e)

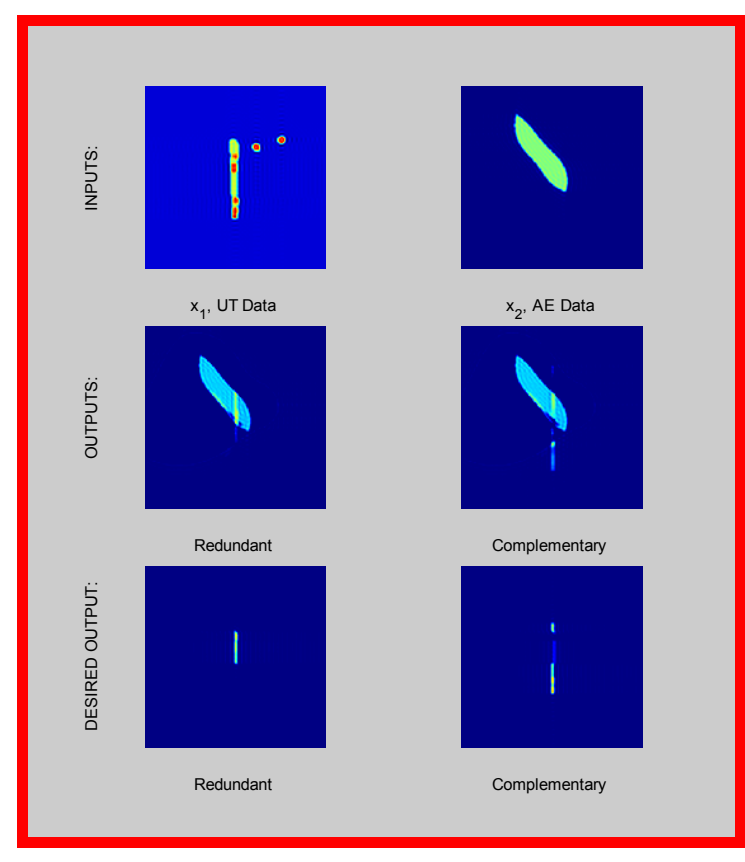

(f)

Figure 16: AE \& UT Combination Trial 1.

Training Data: (a) - (e); Test Data: (f) 
Trial 2: AE \& UT Results

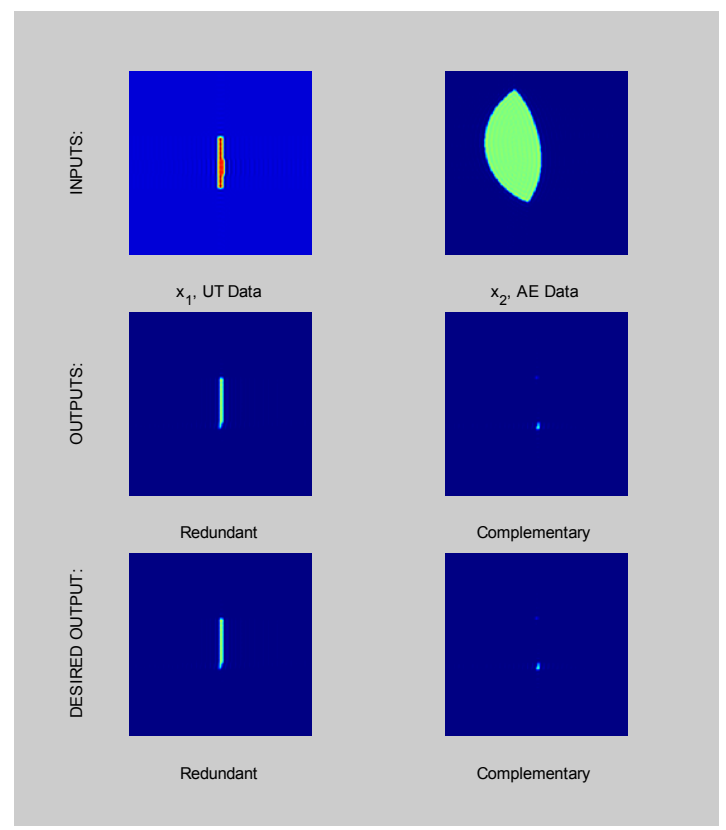

(a)

Trial 2: AE \& UT Results (cont.)

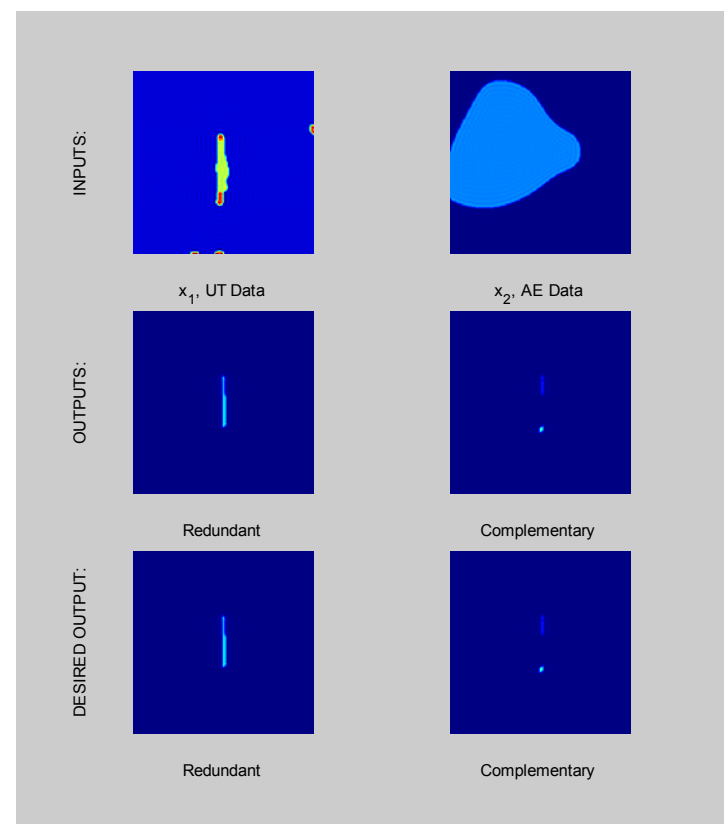

(c)

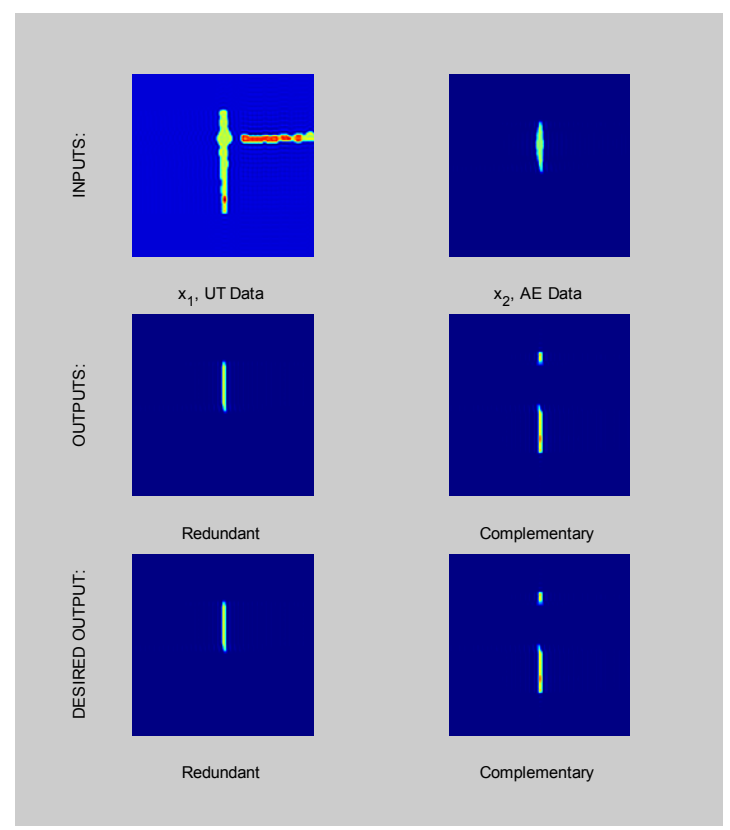

(b)
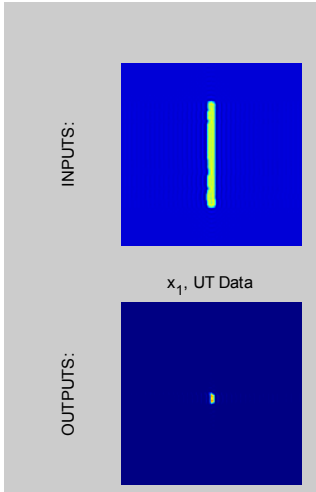

Redundant

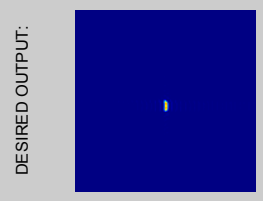

Redundant

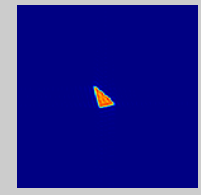

$\mathrm{x}_{2}$, AE Data

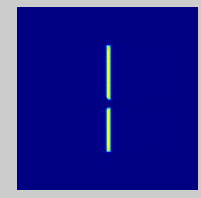

Complementary

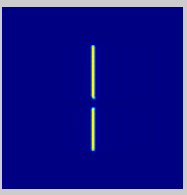

Complementary

(d) 


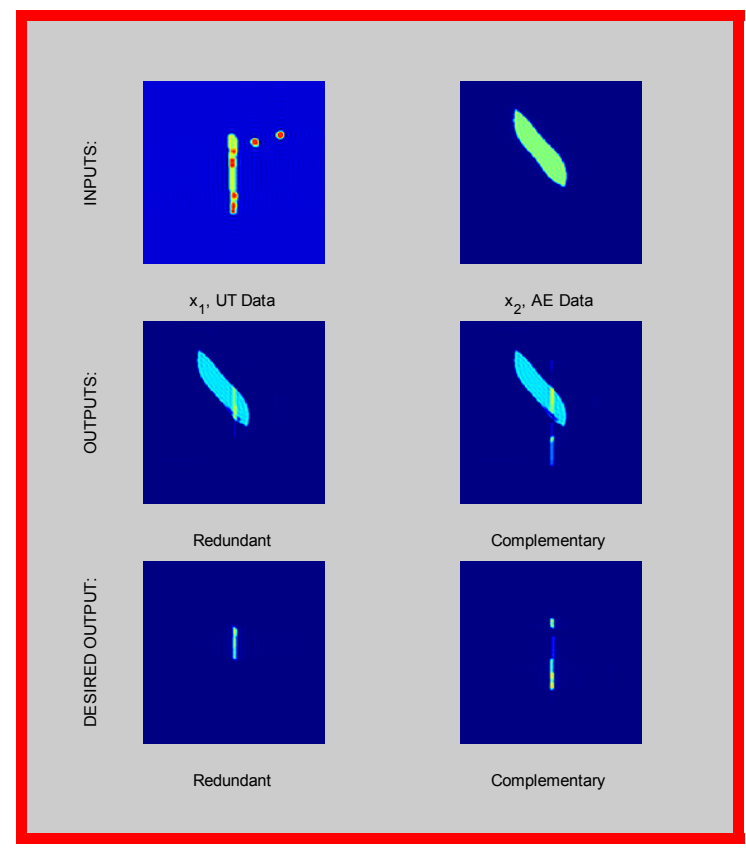

(e)

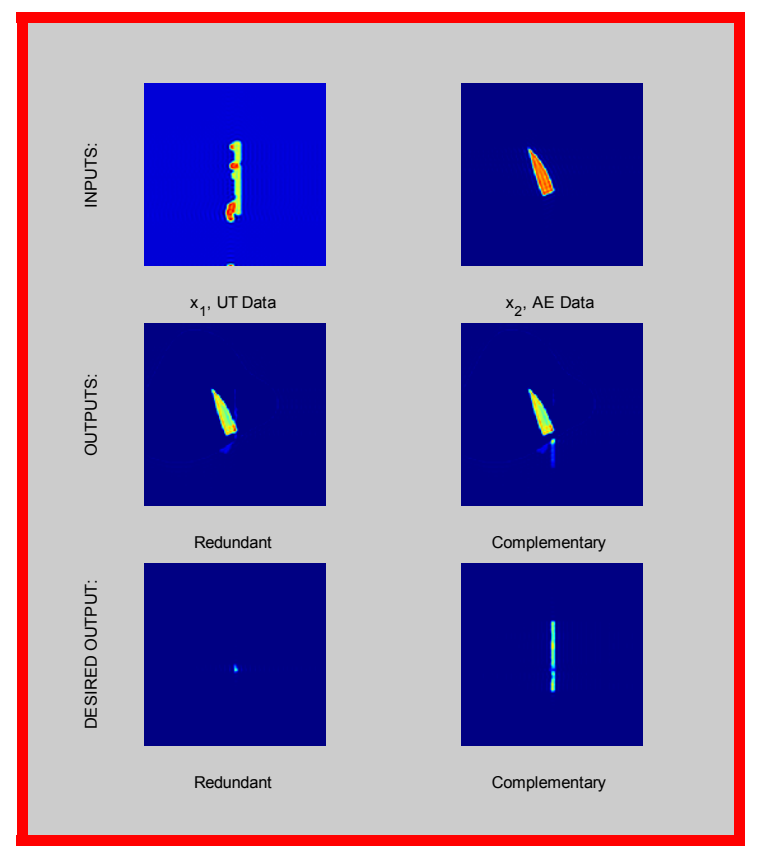

(f)

Figure 17: AE \& UT Combination Trial 2.

Training Data: (a) - (d); Test Data: (e), (f)

\section{Results and Discussion}

\section{Task 3.0 - Design and Development Data Fusion Algorithms}

This report concentrates on fusing information from heterogeneous sensors - MFL, UT, thermal and acoustic imaging NDE signatures that have been obtained from a test-specimen suite have been described in previous reports.

The following observation can be made based on the data fusion results shown in the previous section. There exists significant agreement between the predicted and desired redundant and complementary defect depth related information for all instances of training data - this indicates that the information provided to the neural network is distinct and the resulting matrices are non-singular; and is clearly visible especially the UT and MFL data combinations. The poorest performance occurs for MFL and thermal image data combinations - this is to be expected since MFL images exhibit "blooming" and cannot precisely identify the edges of the defect. This situation is worsened for thermal images where the shallowest defects are barely visible. 
The homogeneous data fusion results shown are summarized in the Figures 18, 19 and 20 for purposes of comparison. In analyzing the results, the mean squared error (MSE) is used as a measure of the difference between images, because it measures how far an estimator is from what it is trying to estimate. A lower MSE indicates predicted images that are more similar to the desired output than images with a higher MSE. In analyzing the training data, the MSE between the desired training output and the actual, or predicted, training output may be analyzed. In analyzing testing results, the MSE between the desired output and the actual, or predicted, testing output may be analyzed. The MSE is useful as a relative performance measure to compare different data sets - its use as an absolute measure of algorithm performance is limited. The mean squared error difference between the predicted and desired fused images for both redundant and complementary information, for test and training data bases is shown. The following observations can be made:

1. Over all three trials, the combination UT and MFL images produces the lowest MSE for both redundant and complementary information. This is to be expected, since quantity of information related to the geometry and location of the defect is present in the following NDE methods in decreasing order: UT, MFL, thermal imaging and AE.

2. For all three NDE data fusion combination, the lowest MSE was obtained in Trial 1, where the maximum amount of training data was present. This allowed the neural network data fusion algorithm to accurately interpolate instances of test data. The exact opposite is true of Trial 3.

3. For almost every data fusion combination and trial number, the redundant information extraction algorithm produced a lower MSE then the complementary information extraction algorithm. This is believed to be the result of the redundant training images containing more overall information then the corresponding complementary images.

4. Overall, the algorithm's predicted results matched the desired output values. The total average of the MSE for all combinations of test data was only 0.0201. Very few outputs did not meet the expectations due to poor input data for that test case. All the test data outputs are highlighted in red, where for the most part, the test data shows excellent results. 


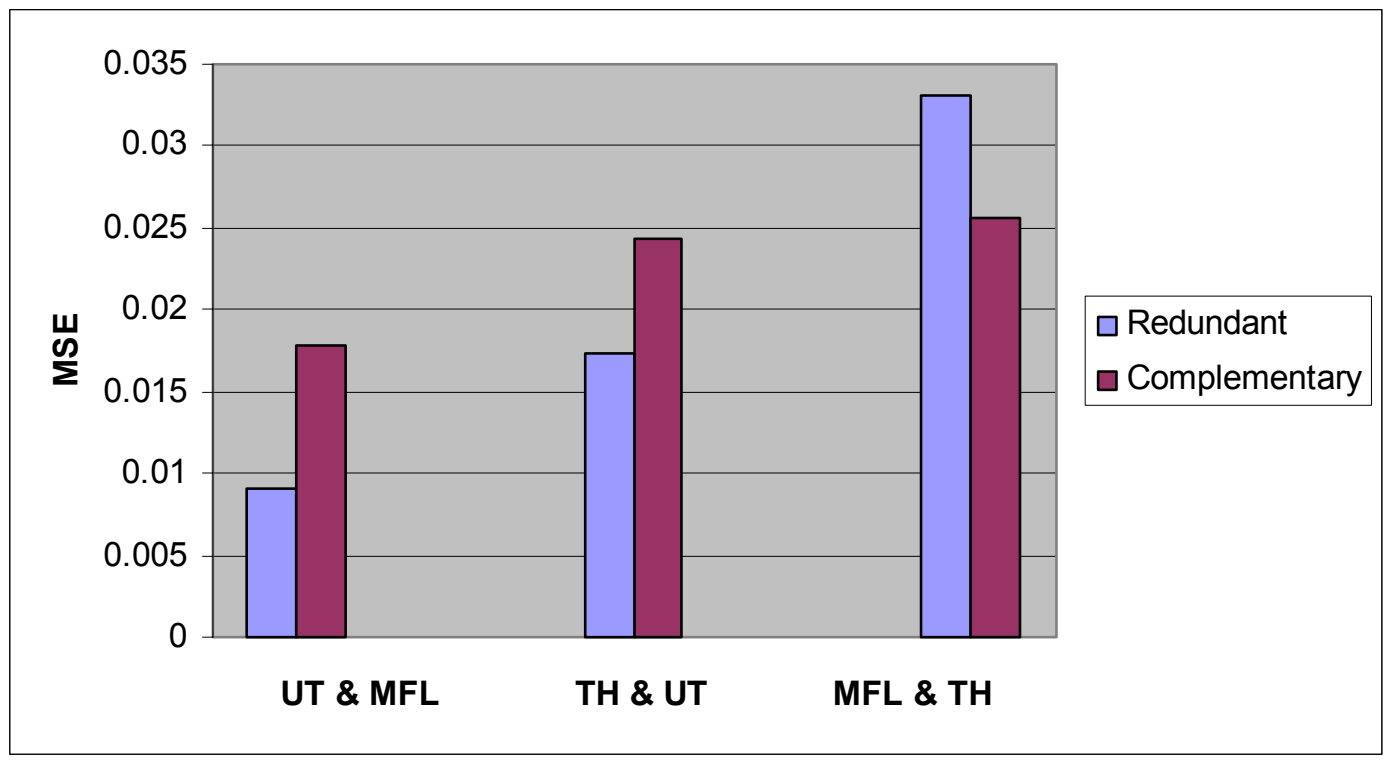

Figure 18: MSE plot of test data in Trial 1 for homogeneous data fusion.

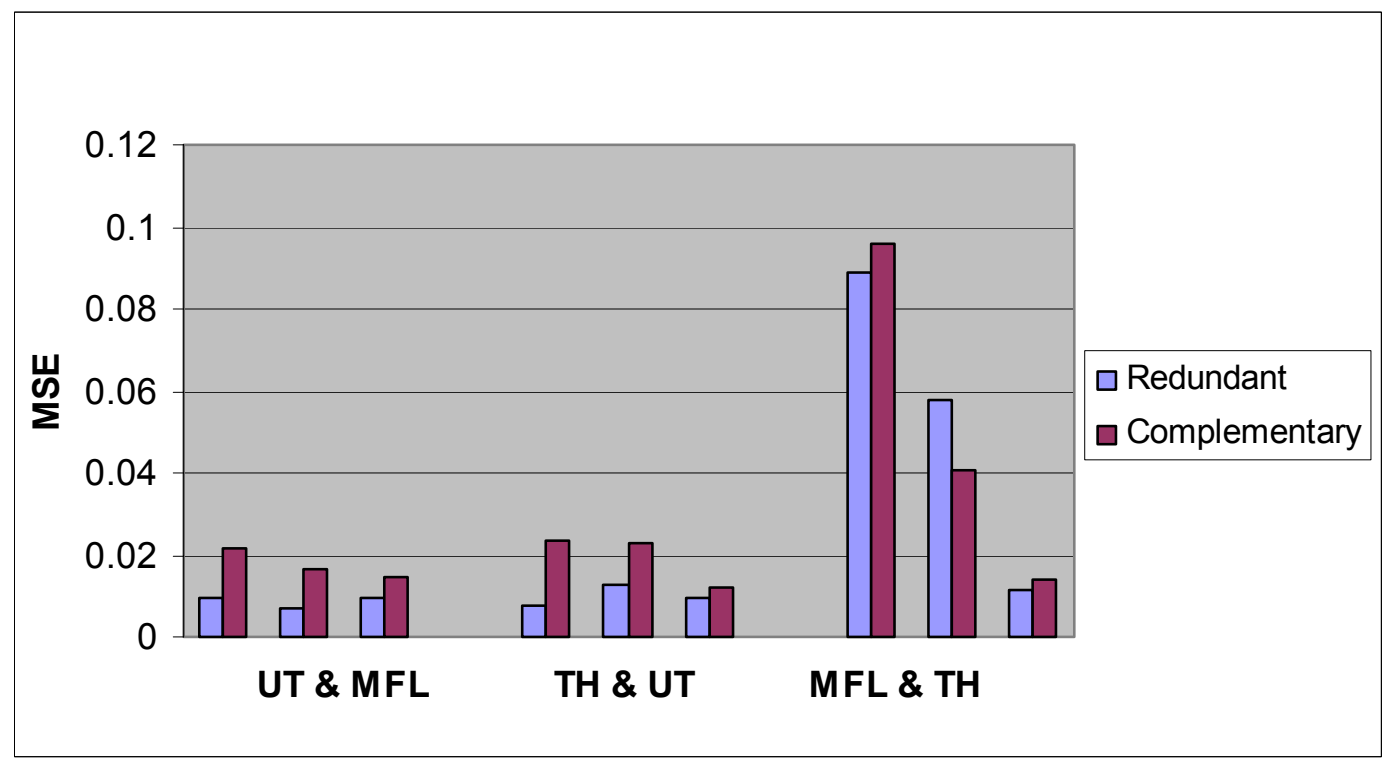

Figure 19: MSE plot of test data in Trial 2 for homogeneous data fusion. 


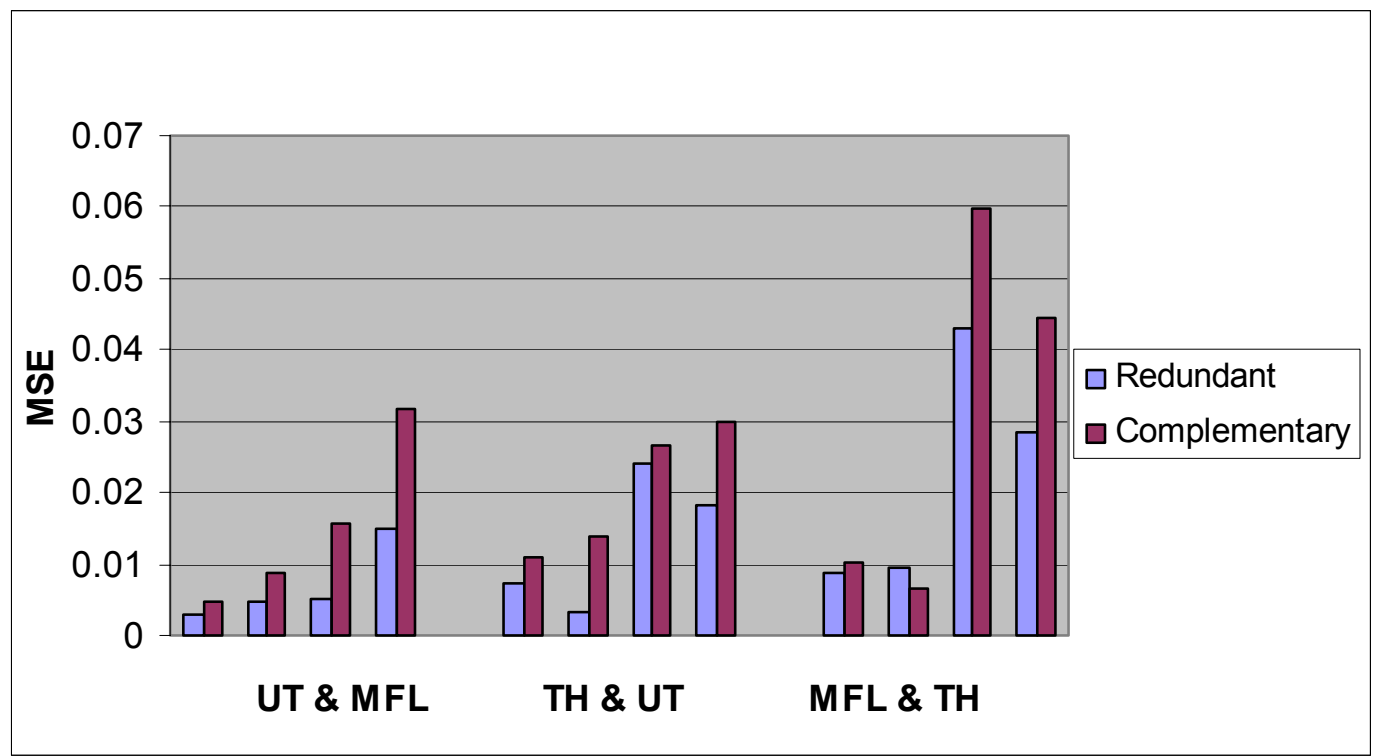

Figure 20: MSE plot of test data in Trial 3 for homogeneous data fusion.

The results from the acoustic emission and ultrasound combination show that both Trials 1 and 2 provided very good training data outputs with an average training output MSE of $2.86 \mathrm{x}$ $10^{-8}$. This indicates that the network was trained well and ready for testing. The subsequent test results are summarized in Figures 21 and 22 where the MSE of test outputs of Trial 1 for redundant and complementary portions are 0.0144 and 0.0156 , respectively. The MSE of test outputs of Trial 2 for redundant and complementary in uniaxial are 0.0121 and 0.0133 , respectively. The MSE of test outputs of Trial 2 for redundant and complementary in biaxial are 0.0187 and 0.0204 , respectively. In both cases the algorithm made an effort to extract the desired information and suppress the unwanted information with a limited amount of training and test data. Although it appears that the heterogeneous data fusion produced better results (lower MSE) than the homogeneous data fusion, with a greatly increased data set, it is expected the heterogeneous data fusion method will exhibit poorer performance. That is, as more data is used for training the heterogeneous network, it is expected that the MSE of testing results will begin to rise to levels closer to those error levels seen in the homogeneous network testing results. This is because the heterogeneous data was pre-processed using a Parzen windows technique to generate an equivalent homogeneous data set, before being fused with other homogeneous data. The accuracy of the resulting data fusion is critically dependent on the accuracy of the pre-processing. 


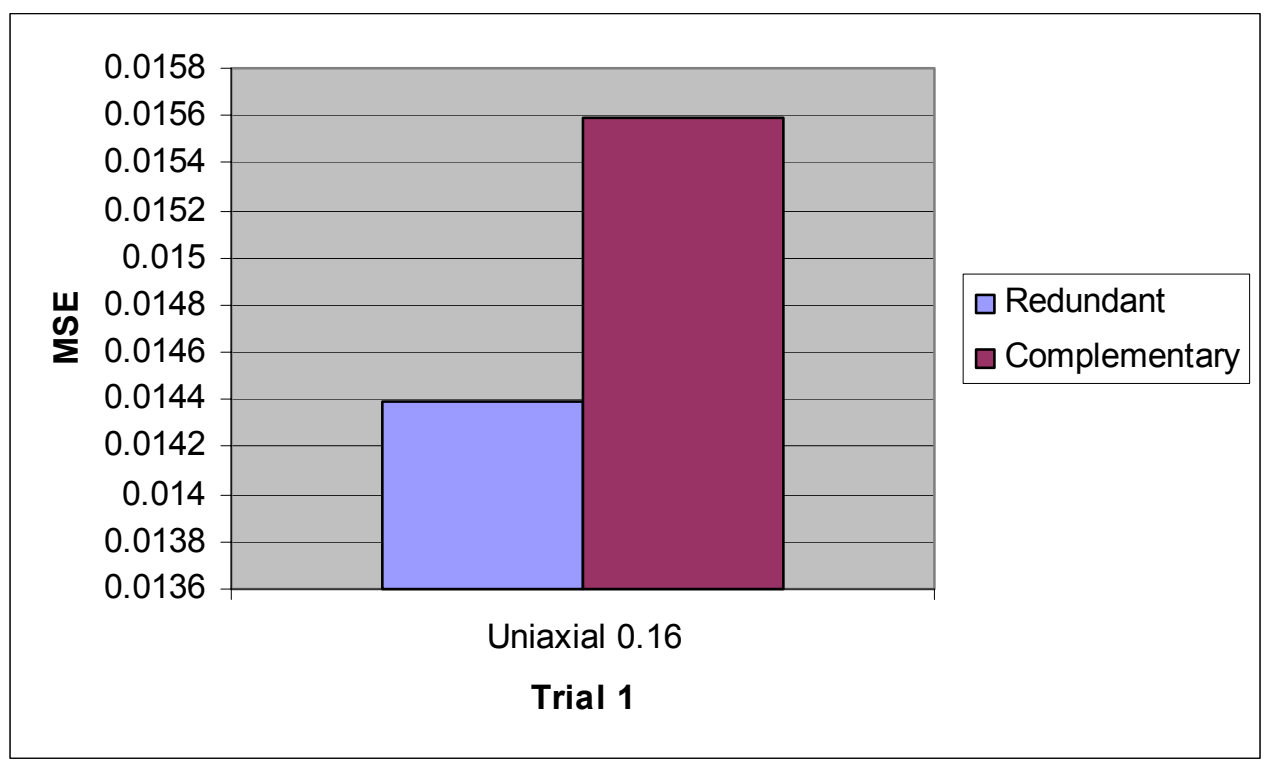

Figure 21: MSE plot of Trial 1 for heterogeneous data fusion.

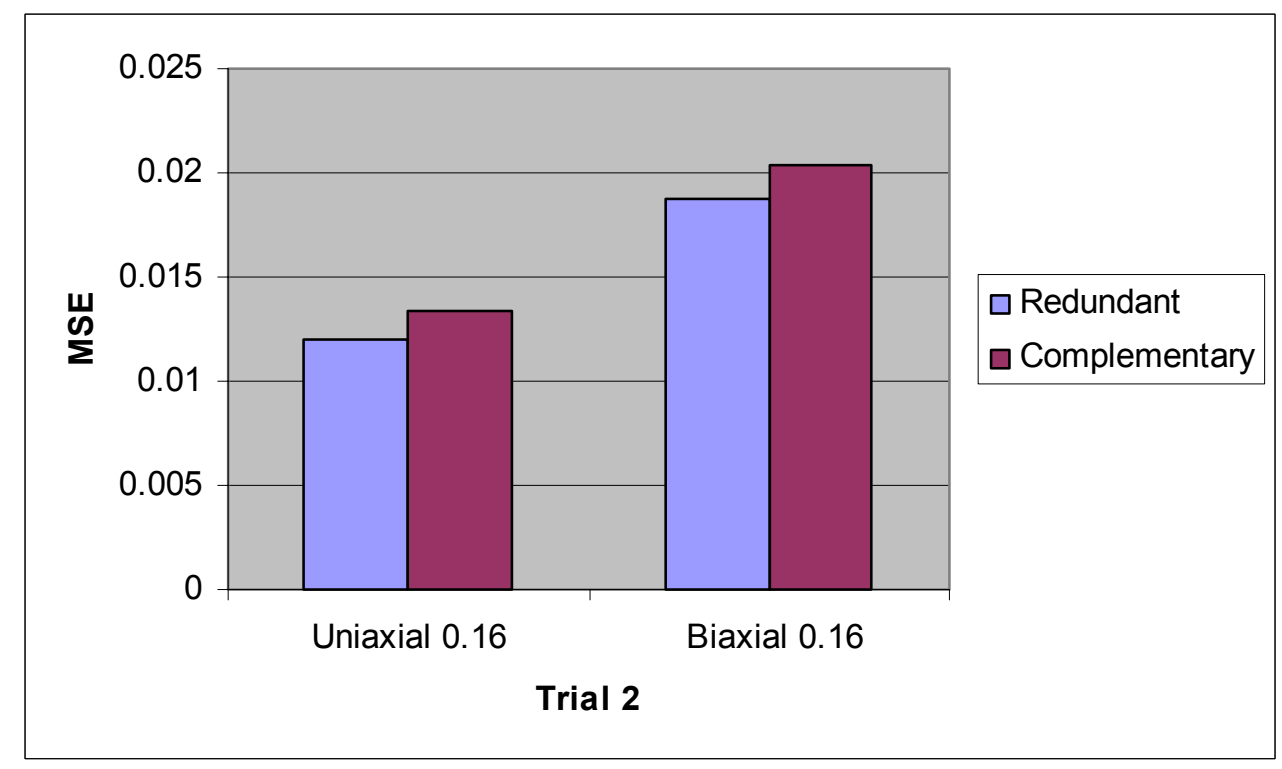

Figure 22: MSE plot of Trial 2 for heterogeneous data fusion. 


\section{Conclusion}

The principal progress during this reporting period involved Task 3.0 - Design and Development of Data Fusion Algorithms.

The important research accomplishments and the conclusions drawn during this research period are in the area of design and development of multi-sensor data fusion algorithms to predict the size of those indications that are identified as anomalies. (Subtask 3.3 - Development of algorithms for defect sizing). The principal contributions during this reporting period and the conclusions drawn from the work are listed below:

1. The design and development of data fusion algorithms for the prediction of specific information fusion measures - redundancy and complementarity - geometric transformations in combination with radial basis function networks and Parzen windows density estimation techniques have been used.

2. The application of the data fusion algorithms to accurately and confidently predict the varying depth profile of surface-breaking pipe wall defects in a gas transmission pipeline - combinations of UT, MFL, thermal imaging and AE NDE data have been fused to predict defect depths in the range of 0.01 " -0.03 " for pipe-wall specimens of thicknesses $5 / 16$ " $-1 / 2$ ". The average MSE between the predicted and desired values for the training data for all the combinations is 0.0028 and for the testing data for all combinations is 0.0201 .

3. The demonstration of the algorithms ability to fuse data from multiple homogeneous and heterogeneous sensors - redundant and complementary information related to the location and size of a pipe-wall defect was predicted using homogeneous data combinations that include UT-MFL, UT-thermal imaging and MFL-thermal imaging; the heterogeneous data combination includes UT-AE.

During the next reporting period, we will address Task 5 -Recommendations for Effective Data Management. 


\section{References}

[1] J.A. Oagaro, Heterogeneous Multi-Sensor Data Fusion Using Geometric Transformations And Parzen Windows For The Nondestructive Evaluation Of Gas Transmission Pipelines, Master's Thesis, Rowan University, Glassboro, New Jersey, December 2004.

[2] R. Duda, P. Hart and D. Stork, Pattern Classification, John Wiley \& Sons, Inc. 2001. 


\title{
List of Acronyms and Abbreviations
}

\author{
$A E$ Acoustic emission \\ DCT Discrete cosine transform \\ $M F L$ Magnetic flux leakage \\ $M L P$ Multilayer perceptron \\ $M S E$ Mean squared error \\ NDE Nondestructive evaluation \\ $R B F$ Radial basis function \\ UT Ultrasonic testing
}

